\title{
Insight into the Cellular Alterations Required for Establishing Opportunistic Pseudomonas aeruginosa Infections
}

Dacie R. Bridge

West Virginia University

Follow this and additional works at: https://researchrepository.wvu.edu/etd

\section{Recommended Citation}

Bridge, Dacie R., "Insight into the Cellular Alterations Required for Establishing Opportunistic Pseudomonas aeruginosa Infections" (2011). Graduate Theses, Dissertations, and Problem Reports. 4700.

https://researchrepository.wvu.edu/etd/4700

This Dissertation is protected by copyright and/or related rights. It has been brought to you by the The Research Repository @ WVU with permission from the rights-holder(s). You are free to use this Dissertation in any way that is permitted by the copyright and related rights legislation that applies to your use. For other uses you must obtain permission from the rights-holder(s) directly, unless additional rights are indicated by a Creative Commons license in the record and/ or on the work itself. This Dissertation has been accepted for inclusion in WVU Graduate Theses, Dissertations, and Problem Reports collection by an authorized administrator of The Research Repository @ WVU.

For more information, please contact researchrepository@mail.wvu.edu. 
Insight into the Cellular Alterations Required for Establishing Opportunistic Pseudomonas aeruginosa Infections

Dacie R. Bridge

Dissertation submitted to the School of Medicine at West Virginia University in partial fulfillment of the requirements for the degree of

\author{
Doctor of Philosophy \\ in \\ Immunology and Microbial Pathogenesis
}

Department of Microbiology, Immunology, and Cell Biology

\author{
Joan C. Olson, Ph.D., Chair \\ Thomas A. Elliott, Ph.D. \\ Karen H. Martin, Ph.D. \\ Lisa M. Salati, Ph.D. \\ Rosana Schafer, Ph.D.
}

Morgantown, West Virginia

2011

Keywords: Pseudomonas aeruginosa, Exoenzyme S, Type III Secretion, Leading Edge, Eukaryotic Cell Migration

Copyright 2011 Dacie R. Bridge 


\title{
ABSTRACT \\ Insight into the Cellular Alterations Required for Establishing Opportunistic Pseudomonas aeruginosa Infections
}

\author{
Dacie R. Bridge
}

The Gram-negative, opportunistic pathogen Pseudomonas aeruginosa $(P a)$ is responsible for causing disease of high morbidity and mortality in individuals who are immunocompromised, suffer from damaged epithelial barriers, and who have cystic fibrosis. However, the underlying host cell compromise that allows the establishment of $P a$ infections remains unknown and is of importance to the development of novel strategies that interrupt $\mathrm{Pa}$ infections. The type III secretion system (T3S) allows the direct translocation of bacterial effectors into the host cell cytosol, and in this study was used first, to identify host cell properties involved in the initiation of $\mathrm{Pa}$ infections, and then second, to dissect the $\mathrm{Pa}$ infectious process. Using the T3S effector ExoS to monitor T3S translocation and the initiation of $P a$ infections in HT-29 epithelial cells, we identified a relationship between leading edge focal complex adhesion properties involved in cell migration and sensitivity to Pa-T3S. Highly migratory T24 epithelial cells were used to further explore the role of host cell migration in $\mathrm{Pa}$ infections, using manipulations of ExoS GTPase activating (GAP) and ADP-ribosyltransferase (ADPRT) activities to manipulate T3S. These studies allowed dissection of the $P a$ infectious process, and found that $P a$ expressing wild type ExoS preferentially bound to the leading edge of T24 cells, where ExoS GAP activity interfered with $\mathrm{Pa}$ internalization, and ExoS ADPRT activity interrupted actin-plasma membrane associations required for T3S translocation. Interestingly, both toxic events limited the $\mathrm{Pa}$ infectious process. Further studies of MTC, MTLn3, and MDCK epithelial cells identified a reciprocal relationship between Rac1 and Rho activation at the leading edge and $\mathrm{Pa}$ internalization and T3S translocation efficiency. Together, the studies in this dissertation highlight the role of actin-plasma membrane associations and Rho-GTPases in directing T3S translocation and the $\mathrm{Pa}$ infectious process, and the ability of $\mathrm{Pa}$ to hijack these factors during cell migration. Our studies in turn are consistent with cell migration properties induced in response to tissue damage being the cellular compromise that leads to the initiation of $\mathrm{Pa}$ infections, and that effectors such as ExoS are able to interrupt these same properties to limit the infectious process and maintain the opportunistic nature of $\mathrm{Pa}$ infections. 
To my parents, Robert and Dacie, my sister, Shanon, and my brother, Roger Your continuous love, support, encouragement, and sacrifices over the years have brought me to where I am today and can never be repaid. I can never express how thankful I am to have you for my family.

To Jon

Your belief in me and my abilities over the years has never ceased to astound me.

Thank you for all of your love and support.

I dedicate this to you with love 


\section{ACKNOWLEDMENTS}

To my advisor, Dr. Joan C. Olson, thank you for your patience, understanding, and guidance throughout my years in your laboratory. Thank you for helping me to achieve my dreams of becoming a scientist. To my committee members: Drs. Thomas Elliott, Karen H. Martin, Rosana Schafer, and Lisa M. Salati, thank you for your time, guidance, and support.

To Drs. Nyles Charon, Thomas Elliott, Slawomir Lukomski, Rosana Schafer, and Scott Weed for use of reagents and equipment. Thank you to Drs. Robert Wysolmerski and Karen H. Martin for assisting me in the development of immunofluorescence protocols and for encouraging my love of microscopy. I will forever be grateful for knowing how to take beautiful images. Dr. Gerry Hobbs, thank you for performing all the statistical analyses presented in this dissertation and for helping me understand statistics.

To Drs. Claudia Rocha and Elizabeth Moore, thank you for your friendship, guidance, and overwhelming support over the years. You have both been an inspiration to me and I was blessed to have followed in your footsteps.

To my dearest friends: Jonathan Hodak, Teri Leavens, Briana Shiley, Fred Findley, Amanda Buskirk, Kelly Miller, Lee Wiley, Heaven Oliver-Kozup, Milinda James, Ricki Krivijanski, Claire Noel, Barbara Pritt, Valerie Watson, Marcella Whetsell, Marsha Gaston, Michael Nguyen, Wendy Lee, Dr. Cathy Yura, and Rita Veasy, thank you for keeping me on track with kind words of encouragement and support. You are like a second family to me and I am blessed to have you in my life.

Thank you to my teachers Bernadette Mendish, Esther B. Ulery, Sue Malarik, and Kimberly McWherter who inspired me to go into science at a young age and for supporting all of my scientific endeavors over the years. To the Department of Microbiology, Immunology, and Cell 
Biology, thank you for the academic and financial support you have provided me while I finished my dissertation. Matthew Novotny, thank for your collaborations on projects included in this dissertation.

To Dr. Jennifer Koehl, you have been a mentor, inspiration, and a wonderful friend. Thank you for inspiring me to go on in Microbiology. I couldn't have done this without your help and guidance over the years. I have been so blessed to have you in my life. Bacteria are so cool!

To all of my family, thank for your love and support over the years. 


\section{TABLE OF CONTENTS}

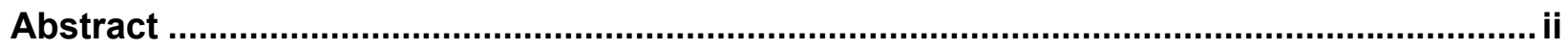

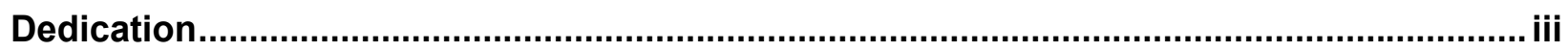

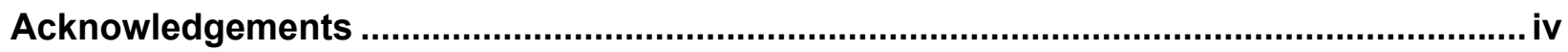

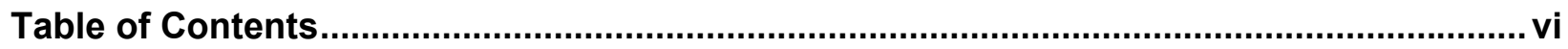

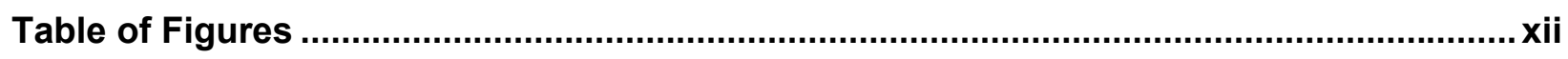

Table of Tables ...........................................................................................................

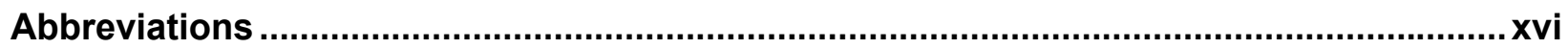

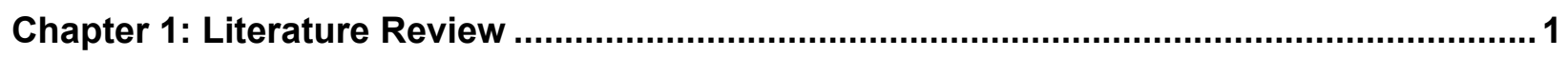

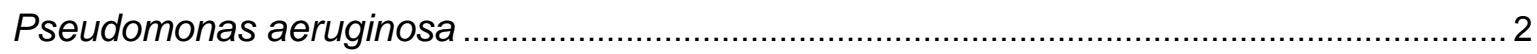

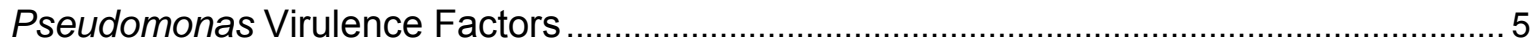

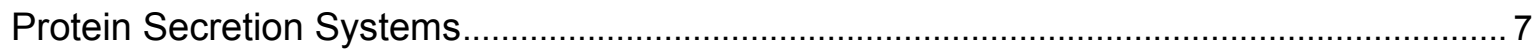

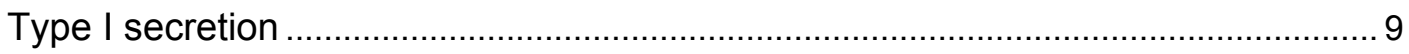

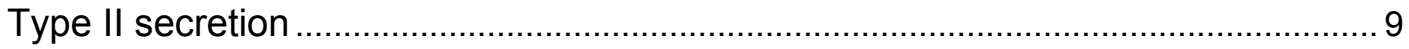

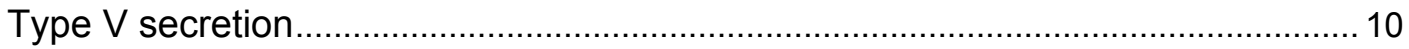

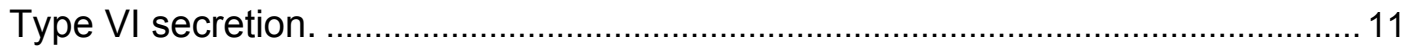

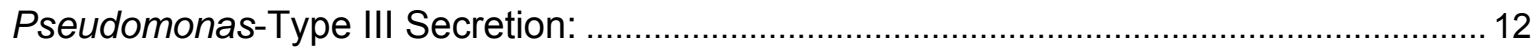

Role of the type III secretion system in infection .................................................... 12

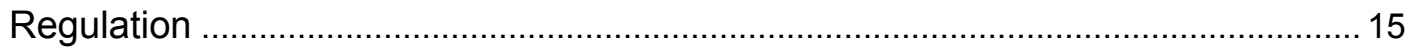

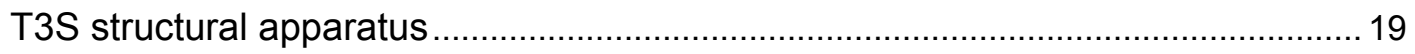

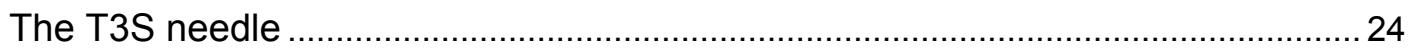

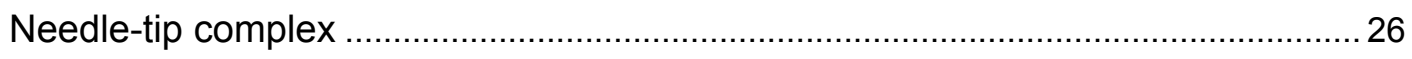

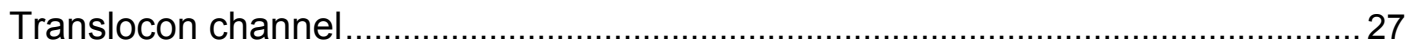




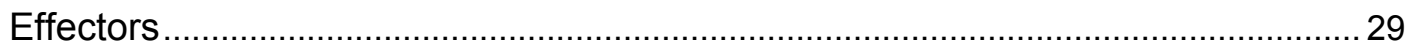

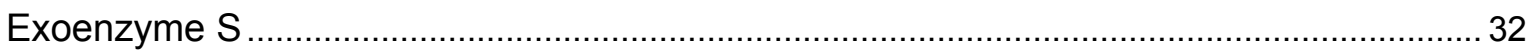

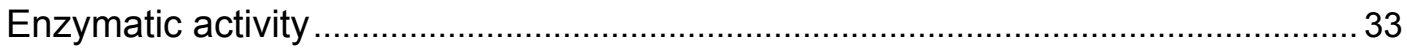

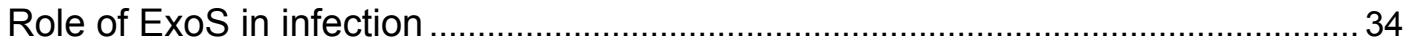

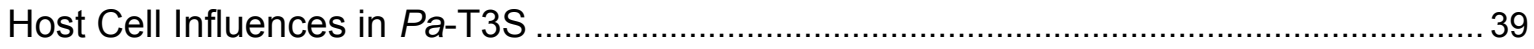

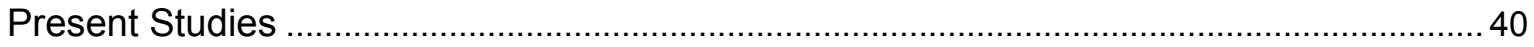

\section{Chapter 2: Role of Host Cell Polarity and Leading Edge Properties in Pseudomonas}

aeruginosa Type III Secretion .................................................................................. 46

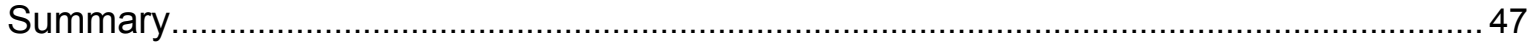

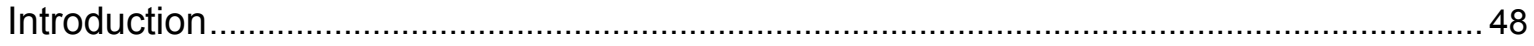

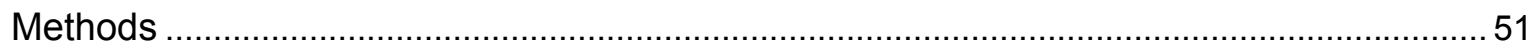

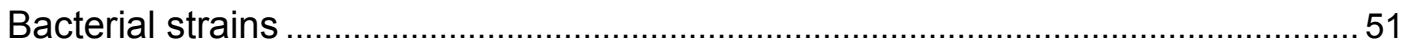

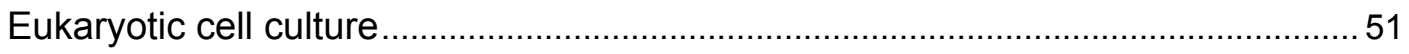

Bacterial-eukaryotic cell co-culture studies and drug treatments .......................... 51

Analysis of eukaryotic cell cytotoxicity, adherence, and Pa-T3S sensitivity ........... 52

Analysis of effects of drug treatments on bacteria ............................................. 53

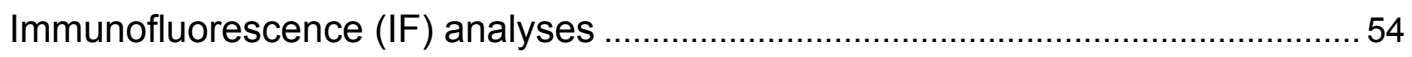

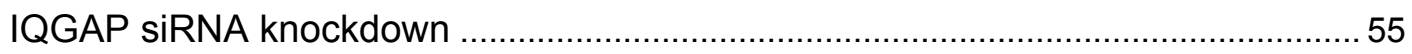

Focal complex, focal adhesion, and LE functional analyses .............................. 55

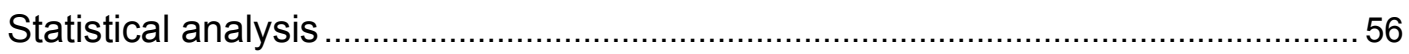

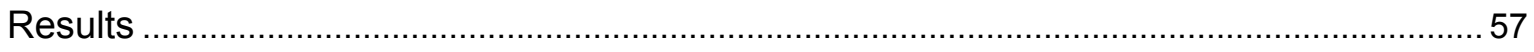

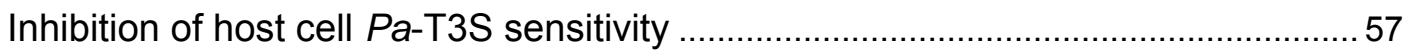

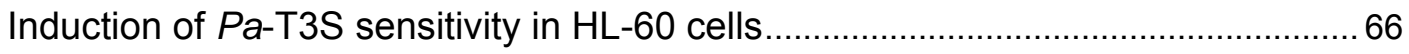

Association of eukaryotic cell adhesion processes with $\mathrm{Pa}-\mathrm{T} 3 \mathrm{~S}$ sensitivity............70 
Examining the type of adhesion mechanism involved in host cell Pa-T3S sensitivity

Examining the relationship between host cell LE properties and $\mathrm{Pa}$-T3S 72

Examining host cell architecture associated with $\mathrm{Pa}-\mathrm{T} 3 \mathrm{~S}$ sensitivity..... .77 HL-60 cell model. .77

HT-29 cell model 80

Discussion 84

Acknowledgements .92

\section{Chapter 3: Gaining Insight into the Opportunistic Nature of Pseudomonas aeruginosa Infections through Manipulations of the T3S Effector ExoS ......................................103}

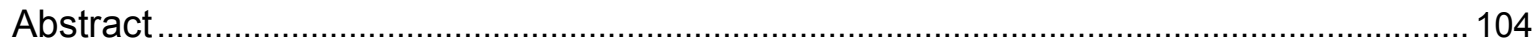

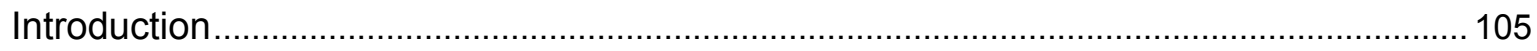

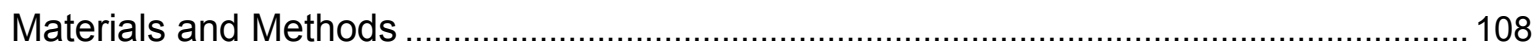

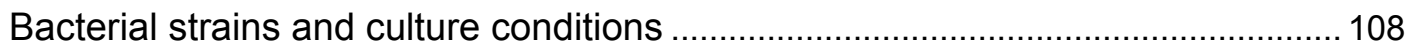

Eukaryotic cell culture and bacterial-eukaryotic cell co-culture conditions............. 108

Analysis and quantification of T3S translocation ............................................ 109

Analysis of T24 cell adherence and morphology............................................ 109

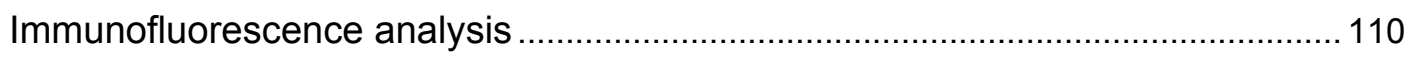

Co-immunoprecipitation of actin with ExoS .................................................... 111

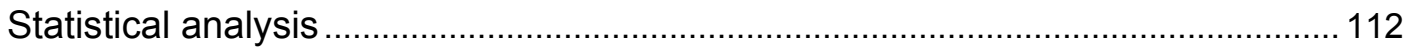

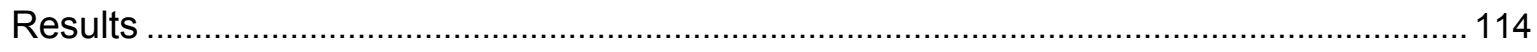

Characterizing the influence of ExoS enzyme activity on T3S

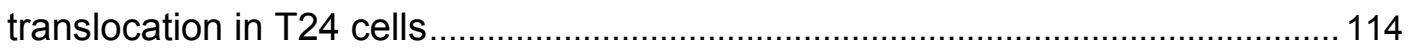

Examining $\mathrm{Pa}$ binding to T24 cells in relation to T3S translocation........................ 118

Examining T3S translocation by $P a$ expressing ExoS enzymatic mutants........... 121 


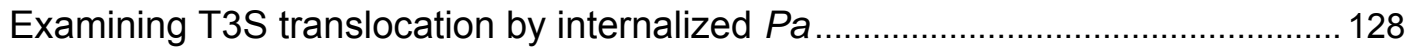

Examining the role of actin in ExoS internalization .............................................. 132

Examining the role of ExoS ADPRT activity in T3S translocation ........................... 136

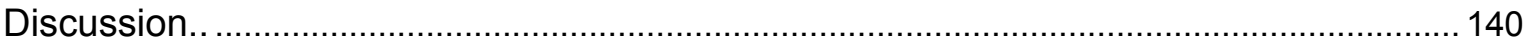

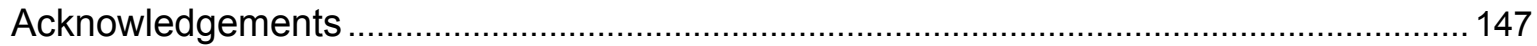

\section{Chapter 4: Differences in Host Cell Migration Lend Insight into Pseudomonas aeruginosa}

Type III Secretion Susceptibility .................................................................................. 151

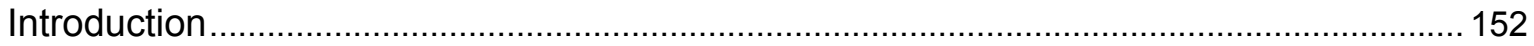

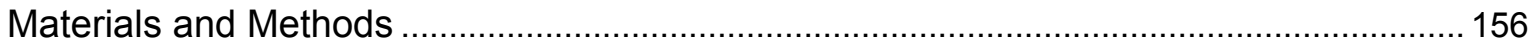

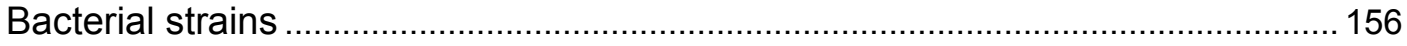

Eukaryotic cell culture and bacterial eukaryotic cell co-culture conditions ............ 156

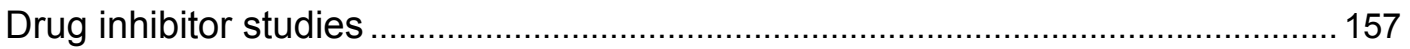

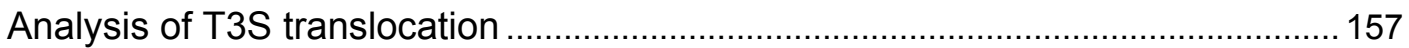

Immunofluorescence analysis of T3S effector translocation and $\mathrm{Pa}$

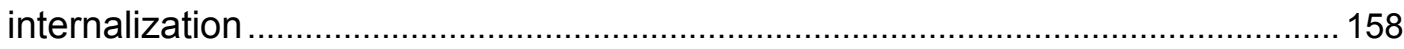

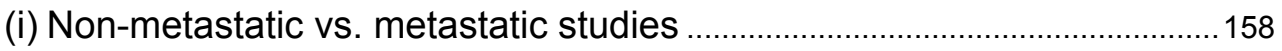

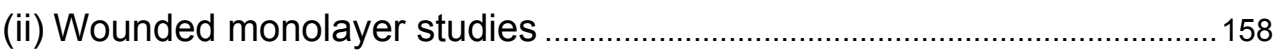

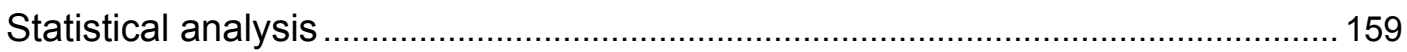

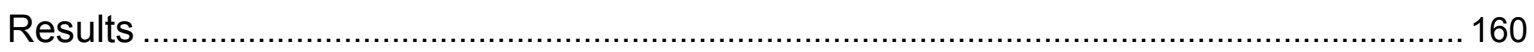

Relationship between host cell migration properties and sensitivity

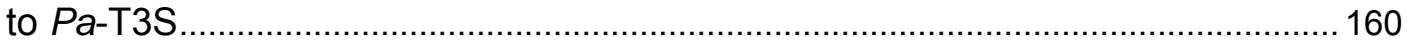

Utilizing ExoS enzymatic mutants to determine differences in Pa-T3S

sensitivity

Characterizing differences in the effects of ExoS GAP activity in MTC and

MTLn3 cells 
Characterizing MDCK cell migration in Pa-T3S ............................................... 180

ExoS GAP and ADPRT activities disrupt MDCK wound healing .......................... 185

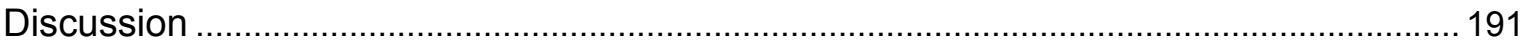

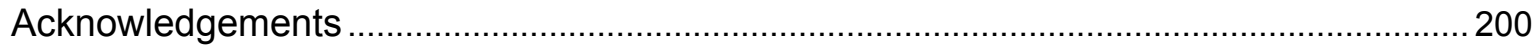

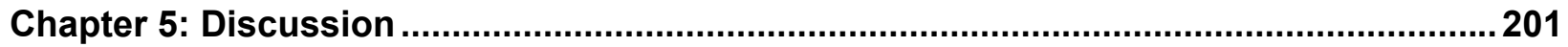

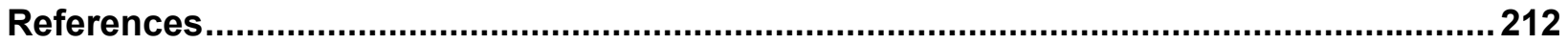

Appendix: Biofilm bacterial diversity: Association with disease severity in contact lens

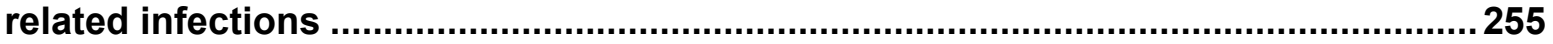

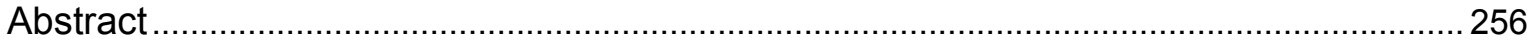

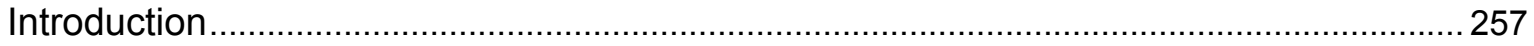

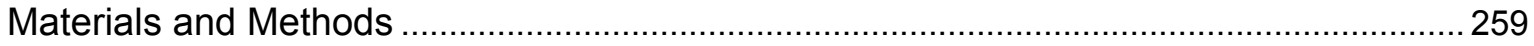

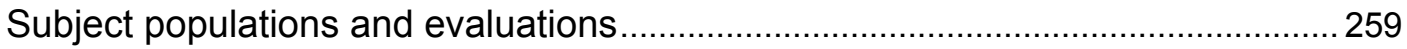

DNA extraction and 16S rRNA gene sequence analysis ....................................259

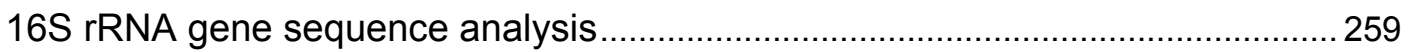

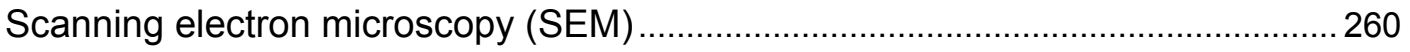

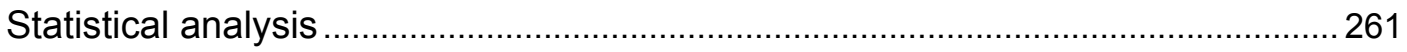

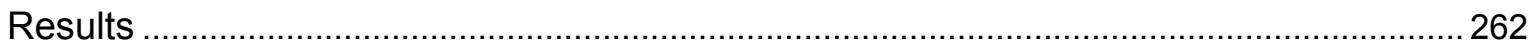

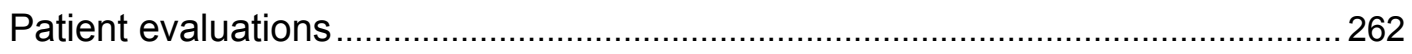

Relationship between bacterial contamination of contact lens cases and

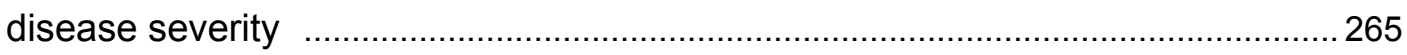

Comparison of bacterial types associated with cases and lenses.......................271

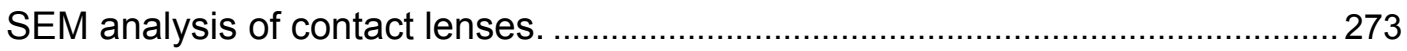

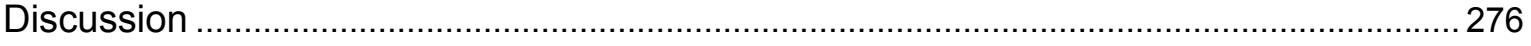

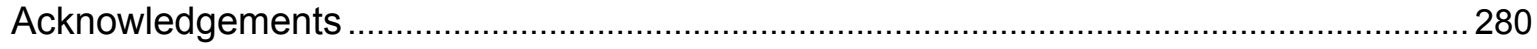


Curriculum Vitae..

Copyrights and Permissions.. 


\section{TABLE OF FIGURES}

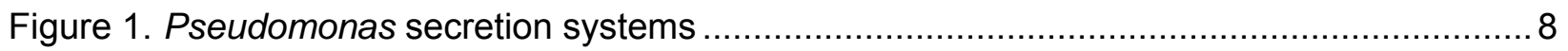

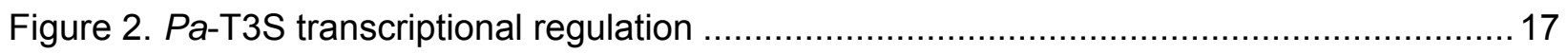

Figure 3. Schematic comparison of the type III injectisome and the flagellum ......................22

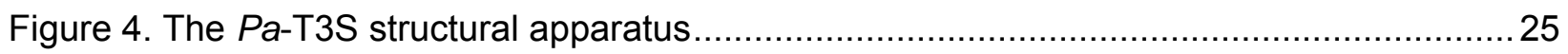

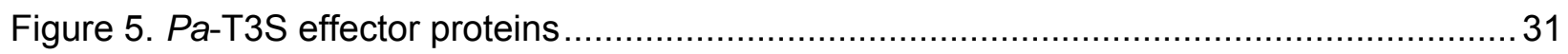

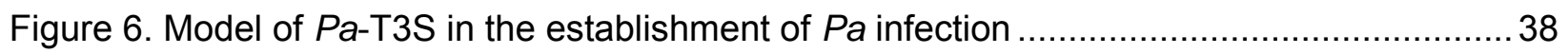

Figure 7. Model of the role of the host cell in the establishment of Pa-T3S infection ............... 44

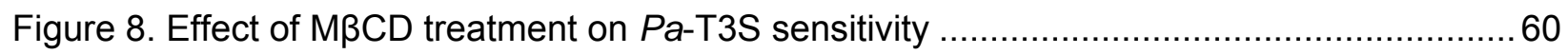

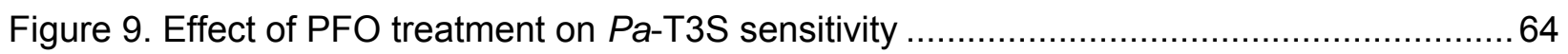

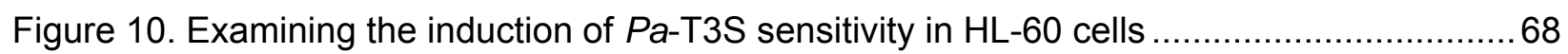

Figure 11. Examining adherence mechanisms and LE properties involved in host cell

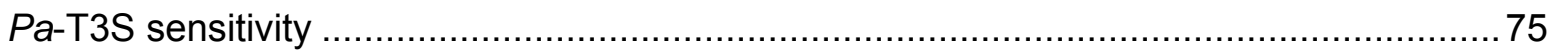

Figure 12. $P$. aeruginosa binding to LE of T3S-sensitive dHL-60 cells ................................ 79

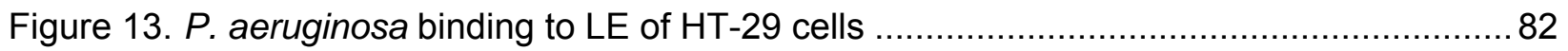

Figure 14. Model for the role of eukaryotic cell properties in Pa-T3S ................................. 89

Figure 15. Influence of ExoS activity on Pa-T3S translocation ...................................... 116

Figure 16. Site of binding of $\mathrm{Pa}$ expressing ExoS constructs to T24 cells ............................ 119

Figure 17. Visualizing the effects of ExoS enzymatic activity on T3S translocation in relation to

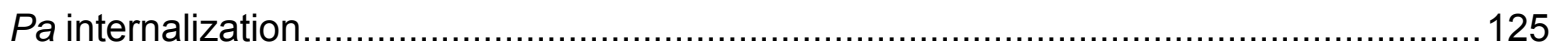

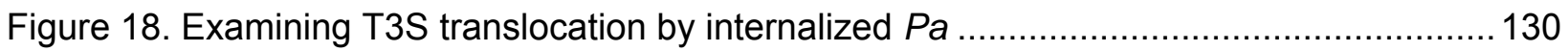


Figure 19. ExoS aligns with actin filaments in T24 cells.

Figure 20. Effect of ExoS ADPRT activity on ERM phosphorylation and localization within

T24 cells. 138

Figure 21. Cell line differences in sensitivity to Pa-T3S 162

Figure 22. Using ExoS GAP and ADPRT mutants to dissect cell line differences. 165

Figure 23. Increased MTLn3 sensitivity is attributed to internalized Pa. 168

Figure 24. ExoS GAP activity alters Rho-GTPase mediated cell morphology differently in MTC and MTLn3 cells 173

Figure 25. Cellular effects of Rho-GTPase inhibitor treatment on MTC and MTLn3

cells 177

Figure 26. Inhibition of ROCK in MTLn3 cells decreased Pa-T3S sensitivity. 178

Figure 27. Inhibition of Rac in MTC cells increased Pa-T3S sensitivity. 179

Figure 28. Length of MDCK wound healing time influenced $\mathrm{Pa}$-T3S sensitivity. 182

Figure 29. $\mathrm{Pa}$ and ExoS align along actin in migrating MDCK cells. 184

Figure 30. ExoS GAP and ADPRT enzyme activity induce independent effects on the MDCK actin cytoskeletal structure during wound healing. 189

Figure 31. Declining visual acuity is associated with contact lens related corneal disease ...... 264

Figure 32. Identification of bacterial types in cases of patients diagnosed with contact lens related corneal disease 267

Figure 33. Relationship between bacterial types and severity of disease 269 
Figure 34. Bacterial biofilm formation on lenses from patients with contact lens related corneal disease

Supplementary Figure S1. Examining the relationship between eukaryotic cell adherence mechanisms and Pa-T3S sensitivity.

Supplementary Figure S2. Examining the role of focal adhesions in host cell Pa-T3S sensitivity......

Supplementary Figure S3. Examining the role of leading edge IQGAP1 in host cell Pa-T3S sensitivity

Supplementary Figure S4. Relationship between HL-60 cell leading edge architecture and Pa-T3S sensitivity. 100

Supplementary Figure S5. Relationship between HT-29 cell leading edge architecture and Pa-T3S sensitivity. 101

Supplementary Figure S6. Staining for external $\mathrm{Pa}$, internal $\mathrm{Pa}$, and ExoS expression 148

Supplementary Figure S7. Visualizing $\mathrm{Pa}$ internalization within membrane vesicles by transmission electron microscopy. 


\section{TABLE OF TABLES}

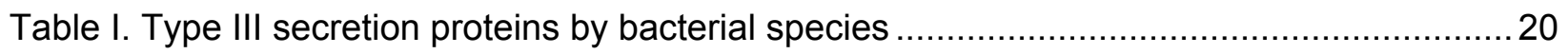

Table II. ExoS mutant activity and cellular toxicity ................................................... 113

Table III. Identification of bacterial types in cases of patients diagnosed with contact lens

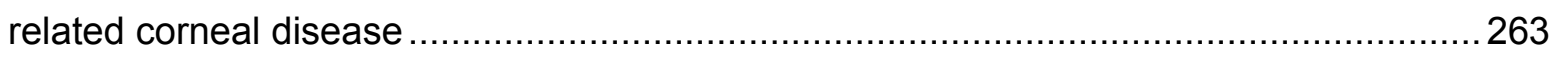

Table IV. Relationship between bacterial types and severity of disease ...........................2 272

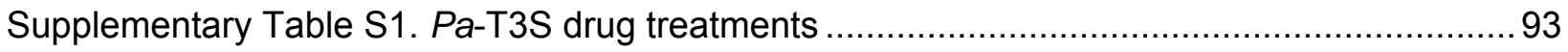




\section{ABBREVIATIONS}

ABC Adenosine triphosphate binding cassette

ACN Acetonitrile

ADP Adenosine diphosphate

ADPRT Adenosine diphosphate-ribsoyltransferase

ATP Adenosine triphosphate

ATPase Adenosine triphosphate dependent motor

ASB Actin stabilization buffer

BSA Bovine serum albumin

C Control

CF Cystic fibrosis

CFTR Cystic fibrosis transmembrane conductance regulator,

CFU/c.f.u. Colony forming units

ColP Co-immunoprecipitation

CTB Cholera toxin B subunit

dHL-60 TPA differentiated HL-60 cells

DI Deionized

DPBS Dulbecco's phosphate buffered saline

dsPFO Pre-pore locked perfringolysin O

ECM Extracellular matrix

EM Electron microscopy

ERM Ezrin, moesin, and radixin

ExoS Exoenzyme S

ExoS-HA Hemagglutinin tagged ExoS

ExoT Exoenzyme T 


\begin{tabular}{|c|c|}
\hline ExoU & Exoenzyme U \\
\hline ExoY & Exoenzyme $Y$ \\
\hline FBS & Fetal bovine serum \\
\hline GAP & GTPase activating protein \\
\hline GAPDH & Glyceraldehyde 3-phosphate dehydrogenase \\
\hline GTPase & Guanosine triphosphatase \\
\hline $\mathrm{h} / \mathrm{hr}$ & Hour \\
\hline HRP & Horseradish peroxidase \\
\hline ICU & Intensive care unit \\
\hline IF & Immunofluorescence \\
\hline IQGAP1 & IQ-motif containing GTPase-activating protein 1 \\
\hline LAMP1 & Lysosomal associated protein-1 \\
\hline LatB & Latrunculin B \\
\hline LE & Leading edge \\
\hline LPS & Lipopolysaccharide \\
\hline$M \beta C D$ & Methyl- $\beta$-cyclodextrin \\
\hline MDCK & Madin-Darby canine kidney \\
\hline MDR & Multi-drug resistance \\
\hline Min & Minute \\
\hline MLCK & Myosin light chain kinase \\
\hline MLD & Membrane localization domain \\
\hline m.o.i/MOI & Multiplicity of infection \\
\hline MTs & Microtubules \\
\hline $\mathrm{OsO}_{4}$ & Osmium tetraoxide \\
\hline $\mathrm{Pa}$ & Pseudomonas aeruginosa \\
\hline PAGE & Polyacrylamide gel electrophoresis \\
\hline
\end{tabular}




$\begin{array}{ll}\text { Pa-T3S } & \text { Pseudomonas Type III Secretion } \\ \text { PERM } & \text { Phosphorylated/phospho-ERM } \\ \text { PFO } & \text { Perfringolysin O } \\ \text { PI3K } & \text { Phosphatidylinositol 3-kinase } \\ \text { PKC } \beta & \text { Protein kinase C } \\ \text { ppm } & \text { Parts per million } \\ \text { rHL-60 } & \text { Undifferentiated HL-60 cells resistant to Pa-T3S } \\ \text { ROCK } & \text { Rho-kinase } \\ \text { rRNA } & \text { Ribosomal RNA } \\ \text { SEM } & \text { Scanning electron microscopy } \\ \text { SDS } & \text { Sodium dodecyl sulfate } \\ \text { sHL-60 } & \text { HL-60 cells acquiring sensitivity to Pseudomonas aeruginosa type III secretion } \\ & \text { during co-culture } \\ \text { sn } & \text { Type III secretion induced culture supernatant } \\ \text { SSPFO } & \text { Monomer locked perfringolysin O } \\ \text { T1S } & \text { Type I secretion } \\ \text { T2S } & \text { Type II secretion } \\ \text { T3S } & \text { Type III secretion } \\ \text { T5S } & \text { Type V secretion } \\ \text { T6S } & \text { Type VI secretion } \\ \text { TEM } & \text { Transmission electron microscopy } \\ \text { TLR } & \text { Toll-like receptors } \\ \text { ToxA } & \text { Exotoxin A } \\ \text { TPA } & \text { 12-O-tetradeconylphorbol-13-acetate } \\ \text { Tetra-methyl rhodamine isothiocyanate } \\ \text { Urinary tract infections } \\ \end{array}$


VAP Ventilator-associated pneumonia

WT Wild type 


\section{Chapter 1: Literature Review}




\section{Pseudomonas aeruginosa}

Pseudomonas aeruginosa $(P a)$ is a ubiquitous, Gram-negative, opportunistic pathogen that has the capability of establishing numerous types of acute and chronic infections associated with severe morbidity and mortality. As an opportunistic pathogen, $\mathrm{Pa}$ infections do not exhibit cell or tissue specificity, and disease occurs only in patients who are immunocompromised, suffer from damaged epithelial barriers, or have cystic fibrosis (CF). The multitude of $P a$ induced infections and the high patient mortality rate associated with this bacterium make it a large human health concern.

During acute infections, $\mathrm{Pa}$ is the leading cause of Gram-negative nosocomial pneumonia that is associated with an approximate $60 \%$ mortality rate, which increases in patients who have ventilator-associated pneumonia (VAP) (253-255). Mechanically-ventilated patients with underlying chronic pulmonary disease who survive an initial episode of $\mathrm{Pa}$ pneumonia are highly susceptible to recurrent infection (294). Risk factors for nosocomial pneumonia include: previous antibiotic treatment which alter normal flora, the duration of mechanical ventilation, length of hospital stay, age, and severity of preexisting disease (summarized in (130)).

$\mathrm{Pa}$ is the third most prominent pathogen in nosocomial catheter-associated urinary tract infections (UTI) (152) and has been reported to cause approximately $35 \%$ of long-term catheter-associated UTIs (266). UTIs are initiated when bacteria are introduced into the bladder at the time of catheterization. Pseudomonas then takes advantage of the damage to the urethral and bladder mucosal surfaces to establish infection (reviewed in (209)). Reoccurrence of UTIs are attributed to biofilm that develops on indwelling catheters to protect bacteria from the host immune response (reviewed in (209))(24)). The presence of iron limiting conditions in the urinary tract, Pseudomonas virulence factors, and high antibiotic resistance of Pseudomonas also appear to contribute to disease severity (reviewed in (209)). 
$\mathrm{Pa}$ bloodstream infections have a crude mortality rate that ranges from $33-61 \%$, placing it second among all pathogens in terms of fatalities $(189,325,335)$. Thirty-five to $50 \%$ of deaths related to $\mathrm{Pa}$ bloodstream infections occur within the first few days of onset. Treatment of these infections is complicated by high levels of antibiotic resistance (74). In this regard, $87 \%$ of patients with a $\mathrm{Pa}$ bloodstream infection have had prior antimicrobial treatment (reviewed in (316)). Catheterization, drainage tubes, and mechanical intubation, all of which disrupt the integrity of epithelial cell barriers, together with septic shock and the initial site of infection are additional risk factors mortality (reviewed in (316)). Underlying disease, such as malignancy, diabetes, and renal failure have been found in $90 \%$ of patients who acquire a $\mathrm{Pa}$ bloodstream infection (reviewed in (316)). Individuals with severe burn wounds are also highly susceptible to $P a$ septicemia due to prolonged healing times and the ubiquitous nature of $P a$.

Another problematic infection associated with $P a$ is ulcerative keratitis. $P a$ has been identified as the most prominent cause of contact lens-related microbial keratitis and corneal ulcer formation, accounting for up to two thirds of infections $(8,43,105,164,239)$. Contact lens wear is a predisposing factor for $\mathrm{Pa}$-induced corneal infection by facilitating $\mathrm{Pa}$ binding to the corneal epithelium, where it can then invade, replicate, and cause severe infection $(86,260,261)$. Due to increased antibiotic resistance, proper treatment of $\mathrm{Pa}$ corneal infections is crucial to prevent decreased vision, permanent damage to the ocular tissues, and in extreme cases, removal of the infected tissue (350).

Pseudomonas infections acquire a different phenotype in the lung of CF patients, where chronic $P a$ lung infections remain the leading cause of death. CF is an autosomal recessive disease that affects multiple tissues and organs due to a mutation in the cystic fibrosis transmembrane conductance regulator (CFTR) (reviewed in (190)). Thickening of mucosal secretions in the lung, due to decreased salt transport, provides an ideal environment for Pa (reviewed in (190)). In the CF lung, $\mathrm{Pa}$ infections are characterized by over-production of the exopolysaccharide, 
alginate, and growth of the bacteria in a biofilm state. CF Pa lung infections tend to last the lifetime of the individual. Studies have shown that $\mathrm{Pa}$ may adapt to CF lung conditions over time, as strains isolated years after lung colonization are remarkably different from the initial colonizing $\mathrm{Pa}$ strains due to down-regulation of virulence factors, such as the type III secretion (T3S) system, flagella, pili, and lipopolysaccharide (LPS) (reviewed in (141))(151, 183). Production of alginate and down-regulation of virulence factors by $\mathrm{Pa}$ is thought to contribute to bacterial survival within the CF lung.

The increased severity and high mortality of opportunistic $\mathrm{Pa}$ infections is due to the propensity of $\mathrm{Pa}$ to develop high levels of antibiotic resistance (42). Multi-drug resistant (MDR) Pa strains have been shown to be transmitted from patient to patient in a clinical setting $(44,274)$ and are associated with particularly severe outcomes $(10,126)$. Risk factors for a MDR $P a$ infection include being immobile, intensive care treatment, and the presence of invasive medical devices, which disrupt protective epithelial barriers. Additionally, it was observed that if patients survived an MDR Pa infection, their functional capabilities were hindered, and these individuals often had to enter a rehabilitation or permanent care facility upon hospital discharge (10).

The ubiquitous nature of $P a$, high antibiotic-resistance, and the ability of $P a$ to form biofilm results in difficulties eradicating the source of infection, particularly in the hospital setting (reviewed in (157)). Pa has been isolated from sources such as unopened distilled water bottles, showers, toothbrushes, water baths, infant feeding basins, cleaning equipment, and respiratory therapy equipment, which supports the notion that infection control is extremely critical in reducing the number of nosocomial $\mathrm{Pa}$ infections (reviewed in (157)). For example, two separate outbreaks of MDR Pa in intensive care units (ICU) could only be terminated when all of the sinks were replaced in the ICU (reviewed in $(157))(23,143)$. Another MDR Pa outbreak in a neonatal ICU resulted in the death of 16 babies and was attributed to the length of 
the nurse's fingernails. Despite newly implemented hygiene control measures after the outbreak, infection was not completely eliminated (reviewed in (157))(210).

Together these studies highlight the diversity and severity of $\mathrm{Pa}$ infection, which is further complicated by incomplete resolution of infection due to MDR, Pa biofilm formation, and the difficulty in eradicating the environmental source of $\mathrm{Pa}$. Another complication in the prevention and treatment of opportunistic Pseudomonas infections is that the host cell factor that allows for the initiation of infection in so many tissue types is unknown, as polarized epithelial monolayers are resistant to $\mathrm{Pa}$ infection. The goal of the current set of studies was to identify the eukaryotic cellular alteration that is responsible for the initiation of Pseudomonas infection for use towards the design of anti-Pseudomonal strategies.

\section{Pseudomonas Virulence Factors}

While MDR is an important factor in resolution of $\mathrm{Pa}$ infections, the pathogenicity of $\mathrm{Pa}$ infections also relates to Pa's vast repertoire of virulence factors (130). The first infections attributed to Pseudomonas were described in the mid-1800s (reviewed in (190)) where Sedillot observed a blue-green color on surgical dressings from patients. Later, rod-shaped structures were observed in pus that had a blue-green tinge. Due to the blue-green tinge associated with infection, Pseudomonas was first given the name Bacillus pyocyaneus by Gessard in 1882. This blue-green tinge was later attributed to production of the pigment pyocyanin, a $\mathrm{Pa}$ metabolite which causes oxidative-stress related damage (reviewed in (161)).

Pseudomonas maintains a broad array of virulence factors that allow it to survive and produce disease at any site within a host, provided that it is not cleared by the immune system. Included amongst its virulence factors are cell-associated: flagella, pili, non-pili adhesins, and LPS, as well as secreted: hemolysins, proteases, and exotoxin A (317). Pa use the propeller, or corkscrew-like, swimming motility produced by flagella to localize to a site of colonization within the 
host and can function in tethering $\mathrm{Pa}$ to epithelial cells (reviewed in (161)). Flagella are immunogenic and activate NF-k $\beta$ via interactions with host Toll-like receptors (TLR) (reviewed in (161)). Pili are responsible for a twitching motility, which allows the bacterium to spread along a surface and contributes to host colonization (reviewed in (161)). Studies by Franklin et al. showed that a piliated $\mathrm{Pa}$ strain was able to adhere to cilia of freshly isolated human tracheal epithelial cells, further highlighting the role of pili in adhesion to host cells (94). The role of flagella and pili in $\mathrm{Pa}$ pathogenesis are supported by studies showing that $\mathrm{Pa}$ strains with mutations in either result in decreased virulence in animal models of infection (reviewed in $(161))(281))$.

LPS, or endotoxin, is a component of Gram-negative bacterial outer membranes and is comprised of: (1) a hydrophobic Lipid A domain, which inserts into the bacterial membrane; (2) a hydrophilic tail made of core polysaccharide; and (3) the O-specific polysaccharide (262). Variability within the O-polysaccharide is used to serotype all Gram-negative bacteria. Currently there are 20 known LPS serotypes in $\mathrm{Pa}$ that can be used to help identify the source of the isolate $(177,252,327)$. For example, serotypes P4, P5, P6, P11, and P12 have been identified as the predominant serotypes in $\mathrm{Pa}$ urinary tract infections, whereas serotype $\mathrm{P} 1$ has been associated with both CF and urinary tract $\mathrm{Pa}$ isolates $(306,327,332)$. Like flagella, LPS is highly immunogenic and contributes to disease through Lipid A activation of TLR4, which results in septic shock (reviewed in (161)).

Alginate is a cell-associated virulence factor produced by $\mathrm{Pa}$ in the CF lung during chronic infection and is comprised of repeating beta-D-mannuronic and alpha-L-glucuronic acid polymers (reviewed in (209)). Mutations in the mucA, mucB, and mucD genes (120) results in production of alginate, which transitions $\mathrm{Pa}$ to a mucoid state. Alginate production by $\mathrm{Pa}$ results in an increase in disease severity, protects $\mathrm{Pa}$ from clearance and antibiotic treatment, and may attenuate the host's immune response to the bacterium. 


\section{Protein Secretion Systems}

In addition to its cell-associated virulence factors, $P a$ is able to secrete multiple factors through five of seven known Gram-negative bacterial secretion systems. Protein secretion by Gramnegative bacteria requires transport across two hydrophobic membranes, the cytoplasmic or inner membrane and the outer membrane, and through an aqueous periplasmic space that separates the two membranes. The five secretion systems utilized by $\mathrm{Pa}$ in its infectious process are diagrammed in Figure 1. 


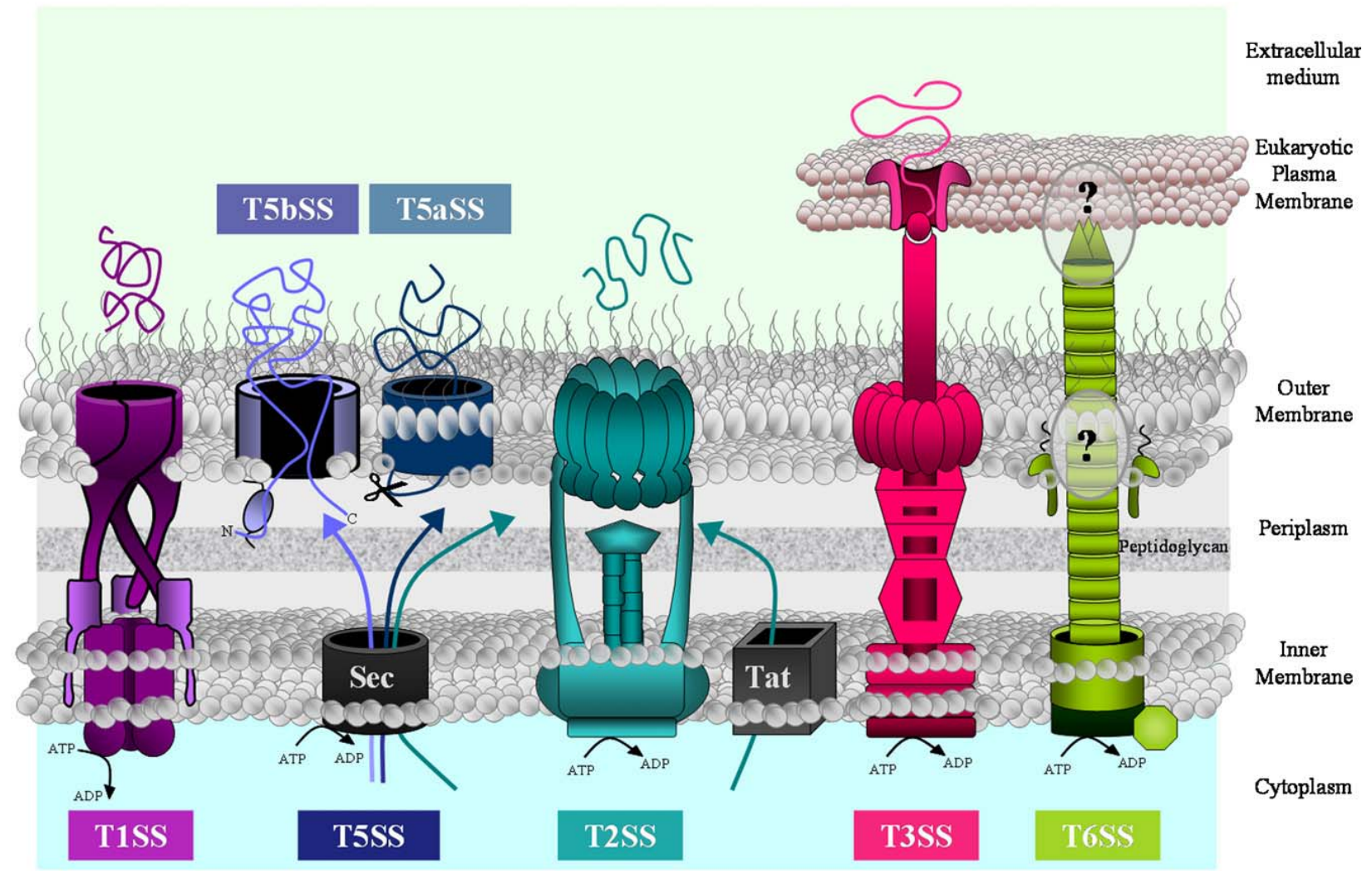

Figure 1. Pseudomonas Secretion Systems. Representation of the five bacterial secretion systems utilized by $P a$.

The image appears by permission of Elsevier Limited. The figure was originally published in the article "Protein secretion systems in Pseudomonas aeruginosa: A wealth of pathogenic weapons" (2010). International Journal of Medical Microbiology. 300(8):534-543 authored by S. Bleves et al. 
Type I secretion

The type I secretion (T1S) system is an adenosine triphosphate (ATP)-binding cassette (ABC) transporter (Fig.1). Proteins secreted through T1S require a non-cleavable, C-terminal secretory signal that interacts with a nucleotide binding domain on the transporter. Upon interaction between the signal sequence and the nucleotide binding domain, a conformational change occurs to promote hydrolysis of ATP. ATP hydrolysis is used to transport the protein through the secretion system and out of the cell. For example, $\mathrm{Pa}$ uses the T1S system for secretion of alkaline proteases and haemophores. Alkaline proteases are responsible for digesting the host protein fibrin, inactivate complement, and are utilized by $\mathrm{Pa}$ in corneal infections (reviewed in (161)). Haemophores bind to haem in haemoglobin and serve as iron scavengers for $\mathrm{Pa}$ in conditions of low iron, which is used for $\mathrm{Pa}$ growth and survival (reviewed in (27)).

\section{Type // secretion}

The Pa-type II secretion (T2S) system is used to secrete the majority of proteins from $\mathrm{Pa}$ and allows protein folding to occur in the periplasmic space prior to traversal across the outer membrane. Proteins are guided to the T2S system by a signal sequence that may contain an unknown conformation secretion motif, as there is no recognized conserved linear motif. The T2S apparatus is similar to the type IV pili assembly apparatus, and like the T3S, is a nanostructure that consists of three components: (1) a protein platform in the inner membrane containing an ATP dependent motor (ATPase); (2) a fimbrillar or pseudopilus structure that pushes the folded proteins out of the cell; and (3) a channel that forms in the outer bacterial membrane (Fig. 1)(reviewed in (27)). Pa utilizes two T2S systems, Xcp and Hxc during infection.

The Pa Xcp system is responsible for the secretion of the following enzymes: (1) elastase or LasB, a metalloproteinase that targets elastin in lung tissue, breaking down tight junctions and 
thus promoting epithelial permeability as well as catalyzing cleavage of surfactant proteins $\mathrm{A}$ and $D$ in the lungs and inactivating complement to decrease the host immune response (reviewed in $(27,161))$; (2) LasA, an elastase that combines its activity with LasB to destroy the extracellular matrix (ECM), enhancing syndecan-1 shedding (reviewed in (334))(211); (3) the aminopeptidase PaAP or protease IV, is important in corneal infection and in cleavage of surfactant proteins in the lung (reviewed in (27)); (4) phospholipase C degrades eukaryotic cell membrane phospholipids, can suppress neutrophilic oxidative burst, and may have a role in inactivation of lung surfactants (reviewed in (187)); and (5) the adenosine diphosphate (ADP)ribosyltransferase exotoxin $A$ (ToxA) (230). ToxA is an $A B$ toxin that interacts with low density lipoprotein receptor-related protein on the host cell surface and following internalization ADPribosylates elongation factor-2, thus inhibiting protein synthesis and causing host cell death $(146,165)$. Currently, the alkaline phosphatase LapA is the only known protein secreted via the Hxc T2S system. Expression of the Pa-T2S system and secreted products is controlled via quorum sensing. Transcription and secretion of proteins by the T2S system only occurs following significant proliferation and accumulation of the bacteria $(228,286,329)$. It is believed that the production of elastases and proteinases, which break down the host cell ECM, may allow for $\mathrm{Pa}$ dissemination from the initial site of colonization (183).

\section{Type $V$ secretion}

Similar to T2S, type $\mathrm{V}$ secretion (T5S) is a two-stage process. Proteins are first transported across the inner membrane utilizing a sec-dependent export apparatus, and then transported across the outer bacterial membrane through a $\beta$-barrel pore (Fig. 1). Proteins may remain attached to the outer surface of the bacterium, or can be secreted into the extracellular medium following proteolytic cleavage. Autotransporters and two-partner secretion are the two subtypes of Pa-T5S (reviewed in (27)). 
Autotransporters contain a signal peptide at the $\mathrm{N}$-terminus, a 'passenger' domain containing the catalytic activity, and a $\beta$-domain at the C-terminus. Once the protein is transported through the inner membrane using the signal sequence, the protein is cleaved and the C-terminal $\beta$ domain inserts into the outer membrane forming a $\beta$-barrel pore. In $P a$, EstA is the only identified autotransporter and is involved in the production of rhamnoplipids, a surfactant with a glycolipid structure that can disrupt ciliary function (139). EstA remains attached to the bacterial surface following secretion and is involved in motility, as estA mutants have been shown to be deficient in twitching, swarming, and swimming motilities (reviewed in (27)).

Two-partner secretion is also a two step process; however it requires two proteins, TpsA and TpsB. Both proteins are transported across the inner membrane using a sec-dependent export apparatus. TpsA is first synthesized as a pre-protein which matures into a pro-protein. TpsB then inserts into the outer membrane where it recruits TpsA. Once in the outer membrane, TpsA is cleaved into a mature protein and can either remain attached to the bacterial surface or can be cleaved and released into the extracellular space. LepA, a protease responsible for activation of NF-k $\beta$, CupB3, which takes part in fimbriae synthesis, and CupB5, a hemagglutinin-like protein, are all secreted by $\mathrm{Pa}$ using two-partner secretion (reviewed in (27)).

\section{Type VI secretion}

The type VI secretion (T6S) system, recently discovered in Vibrio, secretes proteins in a one step fashion and is believed to require host cell contact for protein translocation (Fig.1). While less is known about T6S secretion due to its recent discovery, it is known that there are three T6S system loci in the Pa genome, termed HSI-I, HSI-II, and HSI-III. Hcp1 is the only known substrate secreted by the Pa-T6S system. Notably, Hcp1 has been detected in the lungs of CF patients and the T6S system is believed to play an important role in $\mathrm{Pa}$ lung infections in CF patients (reviewed in (27)). 


\section{Pseudomonas-Type III secretion}

The T3S system is a common virulence mechanism used by numerous Gram-negative bacteria such as Salmonella, Shigella, Escherichia coli, Yersinia, and $\mathrm{Pa}$ (reviewed in (51)). T3S was initially discovered in Yersinia and the T3S structural apparatus includes: (1) the injectisome, a syringe-like nanostructure; (2) a needle; and (3) the translocon, a bacterial formed channel in the host cell plasma membrane (Fig.1). Once fully assembled, a continuous channel is formed between the bacterial cytoplasm and the host cell. This conduit provides bacteria the advantage of manipulating host cell function through the direct translocation of bacterial effector proteins from the bacterial cytoplasm into the host cell, thus bypassing the host immune response. Unlike the T2S system, T3S is sec-independent and T3S effectors act rapidly on the host, independent of significant bacterial proliferation (183). Pa-T3S is believed to be an initiating factor in the establishment of both acute and chronic $\mathrm{Pa}$ infections. Once infection is established using T3S, Pa can then use virulence factors secreted by the other four secretion systems to disseminate from the site of colonization.

Role of the type III secretion system in infection

Clinical studies have shown that patients with acute lung infection caused by T3S-expressing $\mathrm{Pa}$ isolates have a poorer clinical prognosis than those infected with strains not expressing the T3S system $(130,269,296)$. Furthermore, analysis of $P a$ isolates in endotracheal aspirates from patients with respiratory infections, but were not categorized as having VAP, had the ability to secrete type III proteins, and was associated with increased mortality (356). The expression of T3S proteins was also found to contribute to an increase in morbidity and mortality in patients with $\mathrm{Pa}$ bloodstream infections (73). Of 85 patients with a $\mathrm{Pa}$ bloodstream infection, 37 (44\%) had $\mathrm{Pa}$ isolates expressing type III proteins, 16 (43\%) of which progressed to septic shock. Within the first 48 hrs following the onset of septic shock, these patients required vasopressin and hydrocortisone more often, and required higher doses of 
norepinephrine compared to septic patients with non-T3S expressing $\mathrm{Pa}$ isolates. The 30-day crude mortality rate for patients with $\mathrm{Pa}$ bloodstream infections expressing T3S proteins was $46 \%$, an increase of $14 \%$ over the overall crude mortality rate, and an increase of $25 \%$ over patients with isolates that did not express T3S proteins (73). Taken together, this study suggests that risk factors for increased mortality in $\mathrm{Pa}$ bloodstream infections should include the T3S system (73).

In vivo animal studies have shown that $\mathrm{Pa}$ strains with mutations in T3S regulation or the T3S secretion apparatus are less virulent than isogenic wild type strains (170, 204, 333). Moreover, the role of the T3S system in infection was found in both in vivo mouse studies and in epithelial and phagocytic cell line studies where deletion of T3S needle tip protein pcrV (or IcrV from Yersinia) resulted in attenuation in virulence (reviewed in (279)). Like LcrV, passive and active immunization against PcrV was demonstrated to protect against Pa-T3S infection (142, 212, 282). PcrV has been shown to be expressed by most clinical isolates, furthermore, infection with a $\mathrm{Pa}$ strain expressing PcrV without the T3S effectors ExoS or ExoU still results in infections associated with high morbidity and mortality $(1,75,269,356)$. Therefore, the Pa-T3S system and the needle tip protein PcrV make attractive targets for anti-Pseudomonal therapy.

Expression of $\mathrm{Pa}$-T3S during infection was believed to be regulated by the nature of the $\mathrm{Pa}$ infection itself, in that expression of T3S genes occurred primarily in acute infections, while alginate and biofilm genes predominate during chronic $\mathrm{Pa}$ infections $(117,323,344)$. It is postulated that the $\mathrm{Pa}$-T3S system is involved in the initial stages of $\mathrm{Pa}$ infection and is responsible for tissue damage and protection against host immune factors (191). As a chronic $\mathrm{Pa}$ infection develops, T3S is believed to be down-regulated. However, studies over the last several years indicate that T3S may have a more prominent role in chronic Pa lung infections than previously thought $(57,208,269)$. 
In this regard, it is believed that CF patients initially acquire $\mathrm{Pa}$ from the surrounding environment where it is ubiquitous (265). Jain et al showed that $90 \%$ of environmental strains were identified as T3S positive while only $12 \%$ of $P a$ CF isolates were T3S positive (151). This observed difference between T3S phenotypes from environmental and CF isolates does not seem to be due to a lack of T3S effector genes, as there was no difference in the distribution of effector genes (82). Consistent with a low percent of CF isolates expressing T3S, several in vitro studies showed that only a limited number of $\mathrm{Pa} C F$ isolates were able to effectively secrete T3S effectors under T3S-inducing conditions as well as induce T3S-dependent cell death $(55,57,269)$. This T3S negative phenotype by CF isolates could be reversed to a T3S positive phenotype through overproduction of the T3S regulator ExsA, thus indicating that T3S could still be functional under certain conditions (55). Two additional clinical studies have detected serum antibodies to T3S components from both adolescent and adult CF patients, indicating that T3S was still present and potentially functional during the later stages of chronic infection $(18,213)$. The presence of Pa-T3S was also found in the co-culture biofilm of CF isolated airway epithelial cells. In this instance, mutation of Pa-T3S genes resulted in reduced biofilm toxicity $(13,169)$. To determine what occurs between initial Pa colonization and later time-points, two independent studies collected serial $\mathrm{Pa}$ isolates from CF patients over a three or a 15 year period $(151,183)$. Both studies showed that the later $\mathrm{Pa}$ isolates were clonal descendents from the initial T3S positive colonizing isolates. While these isolates gradually lost T3S capabilities as time progressed, some of the later isolates from the three year study remained T3S positive, possibly indicating a reason for positive serum antibodies to T3S components (151).

Taken together, these results indicate that CF patients acquire $\mathrm{Pa}$ with functional T3S systems from the environment. Once inside the CF lung, $P a$ alters its virulence profile, where T3S genes become down-regulated and alginate and biofilm related genes become up-regulated (151, 
183). While T3S expression has been shown to be gradually lost during a chronic infection, the presence of T3S in a small percent of isolates indicates that the reciprocal regulatory mechanism between T3S genes and biofilm genes is not $100 \%$ mutually exclusive as was previously hypothesized. More importantly, these studies show that $\mathrm{Pa}$ isolates expressing the T3S system are present during initial $\mathrm{Pa}$ colonization of the CF lung, and thus support the role of T3S in the establishment of infection (48). What remains unknown is why a small percent of $\mathrm{Pa}$ isolates retain their T3S properties during the chronic stage of infection.

$\mathrm{Pa}$-T3S is believed to be an initiating factor in the establishment of both acute and chronic $\mathrm{Pa}$ infections and can be induced in vitro under low calcium conditions or in vivo by host cell contact $(92,311,315)$. As such, developing an understanding of the Pa-T3S translocation process is integral to the development of therapeutic strategies that interrupt this early stage in the $\mathrm{Pa}$ infectious process (reviewed in (129))(322). A great deal is known about the regulation, structural apparatus, and effectors of the Pa-T3S system; however, how the translocon channel inserts into the membrane and how the host cell contributes to Pa-T3S remains unknown. Therefore, the goal of the studies presented in this dissertation was to identify the eukaryotic cellular alteration that facilitates T3S translocon insertion into the host cell membrane which is responsible for the initiation of $\mathrm{Pa}-\mathrm{T} 3 \mathrm{~S}$ and the establishment of $\mathrm{Pa}$ infection.

\section{Regulation}

The Pa-T3S regulon is chromosomally located and includes more than 42 regulatory, structural, and secretory loci contained within five operons (92). Genes encoding translocated effectors and chaperones are located at different sites on the $\mathrm{Pa}$ chromosome (Fig. 2A). ExsA, a transcriptional activator, is the master regulator of Pa-T3S $(37,92,93)$. ExsA is a member of the AraC/XylS family, and as part of the ExSADCE regulatory cascade, activates transcription by binding to 10 identified promoters containing the consensus sequence TNAAAANA (Fig. 2A) $(313,342,348)$. Transcription of T3S genes is blocked by several factors including the anti- 
activator ExsD, which binds to the N-terminus of ExsA (Fig. 2C, left panel), PtrA, which blocks the functional activity of ExsA, and MgtE, which is proposed to block T3S cytotoxicity via its structure $(14,60,123,203)$. The proposed model for transcriptional regulation is such that when $\mathrm{Pa}$ contacts the host cell or is in the presence of low calcium, ExsE is secreted by the bacteria (Figs. 2B and $\mathrm{C}$, right panel). Decreased levels of ExsE free ExsC, an anti-antiactivator, to bind to ExsD. Binding of ExsC to ExsD, disassociates ExsD from the inactive complex it forms with ExsA and allows ExsA to bind to its promoters, activating transcription (Fig. 2C right panel) (reviewed in $(240))(60,258,311,312)$. Activation of this regulatory cascade results in an increased expression of all T3S related genes and additionally results in the production of functional injectisomes (348). This coupling of transcription and secretion results in inhibitory feedback mechanisms that have been shown to occur by secretion of negative regulatory factors, sequestration of co-activators, or sequestration of anti-activators as previously described $(45,59,202,236)$. 
Figure 2. Pa-T3S transcriptional regulation. a) More than 42 structural and functional genes make up the Pa-T3S regulon and are chromosomally located. The genes for the Pa-T3S effector proteins are located at a different site on the chromosome than the ExoS regulon. The ExSADCE operon is responsible for transcriptional regulation of all structural and secretory genes and its mechanism of action is diagramed in c. b) Shows the assembled T3S system and exoenzymes $S, T$, and $U$ bound to their chaperones inside the $P a$ cytosol. Once ExsE is secreted, translocation of the effectors through the needle into the host cell cytosol can occur. c) Left panel: Under conditions of high calcium or in the absence of host cell contact, transcription is blocked by binding of the anti-activator ExsD to ExsA and the anti-anti-activator ExsC to ExsE. Right panel: When calcium levels are reduced or upon host cell contact, ExsE is secreted by the bacteria and ExsC is free to bind to ExsD which causes the release of ExsA. ExsA in turn binds to its promoters, allowing RNA polymerase (RNAP) to bind initiating transcription.

This image appears by permission of the Nature Publishing Group. The figure was originally published in the article "The type III secretion system of Pseudomonas aeruginosa: infection by injection" (2009). Nature Reviews Microbiology. 7: 654-665 authored by A.R. Hauser. 


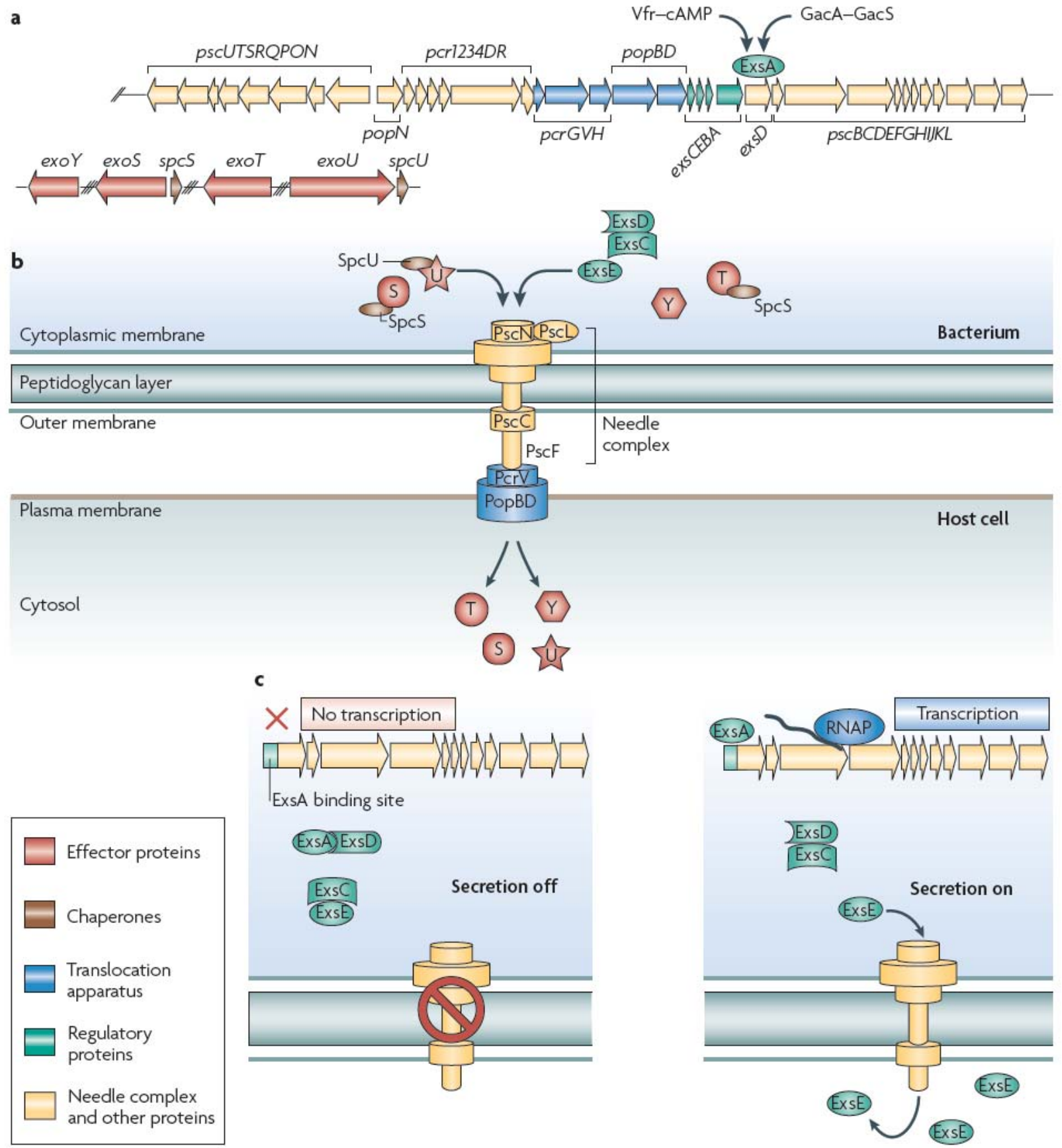




\section{T3S structural apparatus}

There are seven families of T3S systems in Gram-negative bacteria. Originally visualized in Salmonella (168), the T3S injectisome, or needle complex, is a macromolecular structure that spans both the bacterial inner and outer membranes, as well as the aqueous periplasmic space. While the T3S injectisome differs slightly among Gram-negative bacteria, the injectisome is believed to be a common component of T3S systems, as both the genes and organization of the loci are conserved (reviewed in $(308))(29,58,288)$. Some bacterial species such as Salmonella, Shigella, or Burkholderia contain two or more T3S systems for their infectious processes. Differences that occur among bacterial T3S systems relates to the bacteria's environment and infectious processes.

The Pa-T3S system is a member of the Ysc family, Yersinia Yop secretion system (Table I) (reviewed in (308). Despite Yersinia and $\mathrm{Pa}$ belonging to the same family, Yersinia Ysc T3S differs from $\mathrm{Pa}$-T3S in that its structural and regulatory genes are plasmid-encoded (reviewed in (308))(92)). Other T3S systems include: (1) the Inv-Mxi-Spa T3S family of Salmonella, Shigella, and Burkholderia, which triggers actin polymerization and promotes bacterial internalization (Table I); (2) the Ssa-Esc T3S family of Shigella and E. coli that promotes intracellular survival by preventing endocytic trafficking, and allows for adherence to and effacement of intestinal villi, respectively (Table I); (3) the Hrc-Hrp1 and (4) Hrc-Hrp2 families of T3S systems found in plant pathogens; (5) the Chlamydiales system which promotes intracellular survival; and (6) the Rhizobiales system that favors nodule formation or prevents bacterial-root colonization in bacterial-plant symbiotic relationships (reviewed in (308)). 
Table I. Type III secretion proteins by bacterial species.

\begin{tabular}{|c|c|c|c|c|c|c|}
\hline & $\mathrm{Pa}$ & Yersinia & Shigella & Salmonella & Burkholderia & E. coli \\
\hline T3S Family & Ysc & Ysc & $\begin{array}{c}\text { Inv-Mxi-Spa } \\
\text { Ssa-Esc }\end{array}$ & Inv-Mxi-Spa & Inv-Mxi-Spa & Ssa-Esc \\
\hline $\begin{array}{l}\text { Translocon } \\
\text { Proteins: }\end{array}$ & $\begin{array}{l}\text { PopB } \\
\text { PopD }\end{array}$ & $\begin{array}{l}\text { YopB } \\
\text { YopD }\end{array}$ & $\begin{array}{l}\text { IpaB } \\
\text { IpaC }\end{array}$ & $\begin{array}{l}\text { SipB } \\
\text { SipC }\end{array}$ & $\begin{array}{l}\mathrm{BipB}^{*} \\
\mathrm{BipC}\end{array}$ & $\begin{array}{l}\text { EspD } \\
\text { EspB }\end{array}$ \\
\hline $\begin{array}{c}\text { Needle-Tip } \\
\text { Protein } \\
\text { (Chaperone) }\end{array}$ & $\begin{array}{l}\text { PcrV } \\
\text { (PcrG) }\end{array}$ & $\begin{array}{l}\text { LcrV } \\
(\text { LcrG) }\end{array}$ & IpaD & SipD & BipD & EspA \\
\hline $\begin{array}{l}\text { Needle Protein } \\
\text { (Chaperone) }\end{array}$ & $\begin{array}{c}\text { PscF } \\
\text { (PscE) } \\
\text { (PscG) }\end{array}$ & YscF & $\mathrm{MxiH}^{*}$ & Prgl $^{*}$ & & $\mathrm{EscF}^{*}$ \\
\hline Molecular Ruler: & PscP & YscP & Spa32* & $\operatorname{lnv} J^{*}$ & & \\
\hline $\begin{array}{l}\text { Translocon } \\
\text { Chaperone: }\end{array}$ & PcrH & LcrH & & & & \\
\hline Effectors: & $\begin{array}{l}\text { ExoS } \\
\text { ExoU } \\
\text { ExoT } \\
\text { ExoY }\end{array}$ & $\begin{array}{l}\text { YopE } \\
\text { YopK } \\
\text { YopT }\end{array}$ & & & & \\
\hline
\end{tabular}

${ }^{*}$ Not discussed in the text.

From: $(114,201,302)$ 
The T3S injectisome is comprised of more than 20 proteins. The total number of proteins varies by bacterial species, but nine proteins are conserved between known injectisomes. Eight of the structural T3S proteins are also recognized to be shared with the flagellar apparatus (84, 319, 336). Because of the shared components, several studies have shown that the flagellar hookbasal body apparatus and T3S injectisomes share structural and functionality similarity (Fig. 3) $(30,168)$. Despite these similarities, phylogenetically, the flagellar apparatus components cluster separately from their T3S injectisome counterparts, indicating that the two structures have derived from a common evolutionary origin (reviewed in (308)).

Much of what is known about T3S injectisomes has been determined through studies of Salmonella, Shigella, and E. coli $(140,197,284,300)$. The T3S basal body is made of two rings joined by a narrow cylinder which connect to the base of the needle (reviewed in (49)). Cryoelectron microscopy (EM) studies of the Salmonella basal body show that the outer membrane unit, or 'secretin,' contains three rings and is a 15-mer structure. The inner membrane unit is composed of one 24-mer ring (284). The C-terminus of the outer membrane ring is embedded within the outer membrane, while the $\mathrm{N}$-terminus is located within the periplasm (reviewed in (331)). The inner rod, or 'socket,' connects the inner membrane ring with the third outer membrane ring, contains 12 -fold symmetry, and is $2-3 \mathrm{~nm}$ in diameter (reviewed in $(49,331)$. Both periplasmic domains of the outer and inner membrane rings have high structural similarity (reviewed in (331)). Data indicate that a cytosolic or C-ring, which is present within the flagellar apparatus, may be present at the base of injectisome, but this has yet to be identified by cryoEM (reviewed in (49)). The entry point for proteins from the cytosol into the basal body is the cup, which works in conjunction with an ATPase that exports proteins through the secretion apparatus in an ATP-dependent fashion (reviewed in (49))(284). Secretion of proteins from the basal body into the host cell is facilitated by the needle, which is connected to the outer membrane ring and protrudes from the bacterial surface. 
Figure 3. Schematic comparison of the type III secretion injectisome and the flagellum. Schematic of the needle-containing T3S system (TTSS) apparatus (left), as found in bacterial pathogens of animals, and the flagellum (right). The T3S system needle is positioned on outer membrane and inner membrane rings, as is the flagellum. For the T3S system, proteins that are conserved in the flagellar apparatus are indicated without parentheses while those that are not conserved in the flagellar apparatus are enclosed in parentheses. Question marks indicate uncertain localization of proteins. Yersinia proteins are indicated for the T3S system, except for the inner membrane ring, for which Salmonella proteins are denoted. For the flagellar system, proteins that are conserved in the T3S system are indicated without parentheses and proteins that are not conserved in the T3S system are enclosed in parentheses. Effector proteins of the T3S system are thought to travel from the bacterial cytosol through the two rings and the needle and to cross into the host cell cytoplasm through pores formed in the host cell plasma membrane by T3S system translocator proteins. The T3S system needle is $\sim 100$-fold shorter than the flagellum.

The image and figure legend appear with permission from the American Society for Microbiology. The figure is part (A) of a three part figure and was originally published in the article "Process of Protein Transport by the Type III Secretion System" (2004). Microbiology and Molecular Biology Reviews. 68: 771-795 authored by P. Ghosh. 


\section{Host Cell Cytosol}

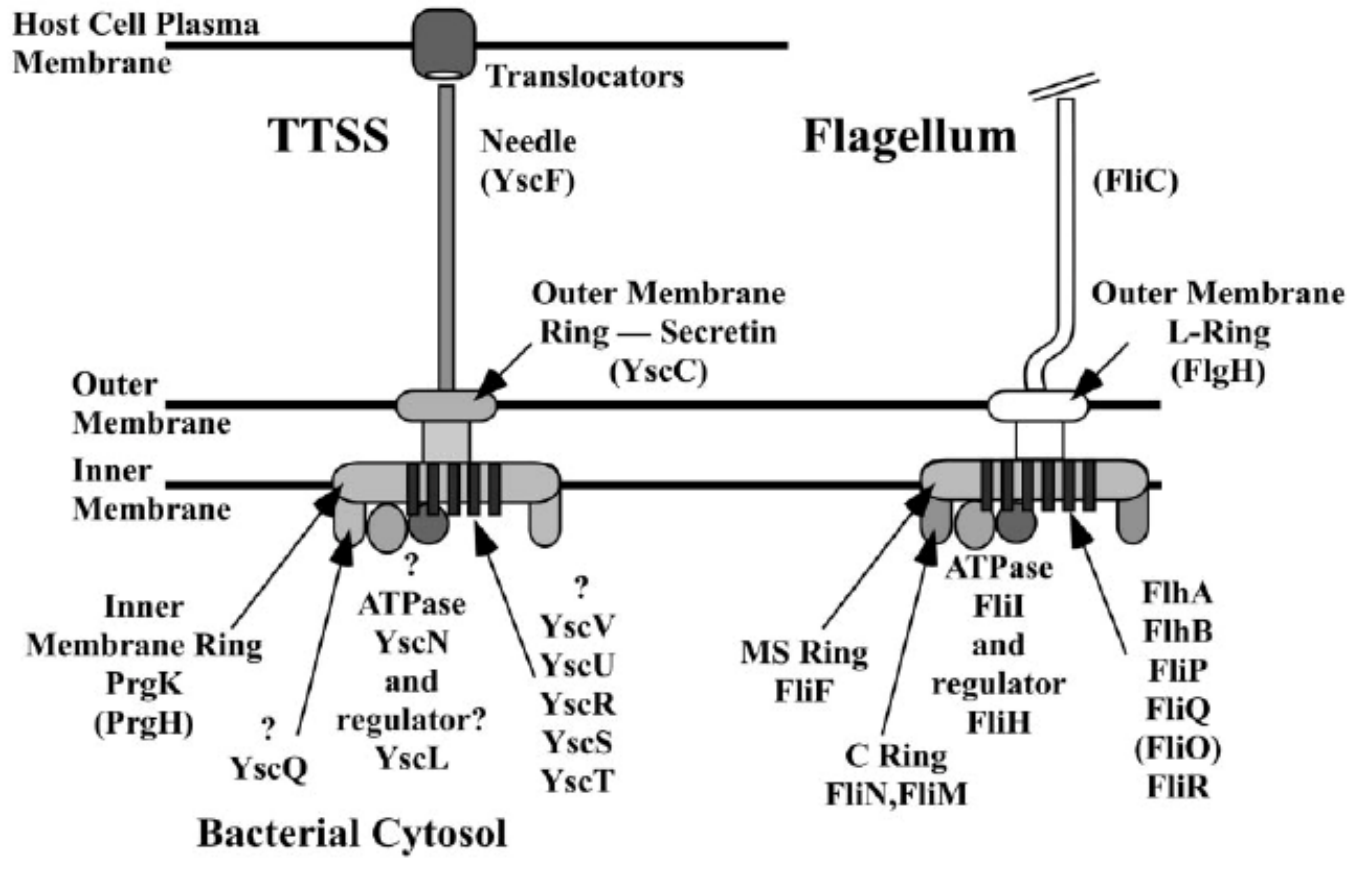


The T3S needle

Specific to $\mathrm{Pa}$, the T3S needle is $60-120 \mathrm{~nm}$ in length, $7-10 \mathrm{~nm}$ in width, $2 \mathrm{~nm}$ in internal diameter, and facilitates the transfer of bacterial effectors into the host cell (Fig. 4) $(229,298)$. The needle is composed of $9 \mathrm{kDa}$ PscF subunits and contains $57 \%$ sequence similarity with YscF, the major protein component of the Yersinia-T3S needle (Table I) (229). Despite sequence similarity, functional complementation studies of a $p s c F$ deletion by $y s c F$ indicate that the PscF and YscF needle proteins are species-specific (229). The role of the needle in effector translocation is supported by studies showing that deletion of pscF results in the loss of needle formation and effector translocation (229).

Needle biogenesis is regulated by a PscE-PscF-PscG complex within the bacterial cytoplasm. Binding of PscF to its two cytosolic chaperones keeps it in a secretion efficient conformation and prevents oligomerization while inside the bacteria $(241,248)$. T3S needle length is highly regulated and is postulated to be controlled by PscP, a homologous protein to YscP, the "molecular ruler" in Yersinia-T3S needle formation (Table I) $(3,153,214)$. It is hypothesized that the two ends of YscP act as an anchor, where one end is tethered to the basal body and the other tethered to the growing needle. Upon completion of needle synthesis, and when the needle equals the length of the YscP protein, a signal is believed to be transmitted to the basal body, thereby terminating YscF secretion and initiating secretion of other proteins (153). Electron microscopy and crystal structure analysis of Yersinia needles have revealed a distinct structure at the tip of the needle $(36,63,67,216)$. 


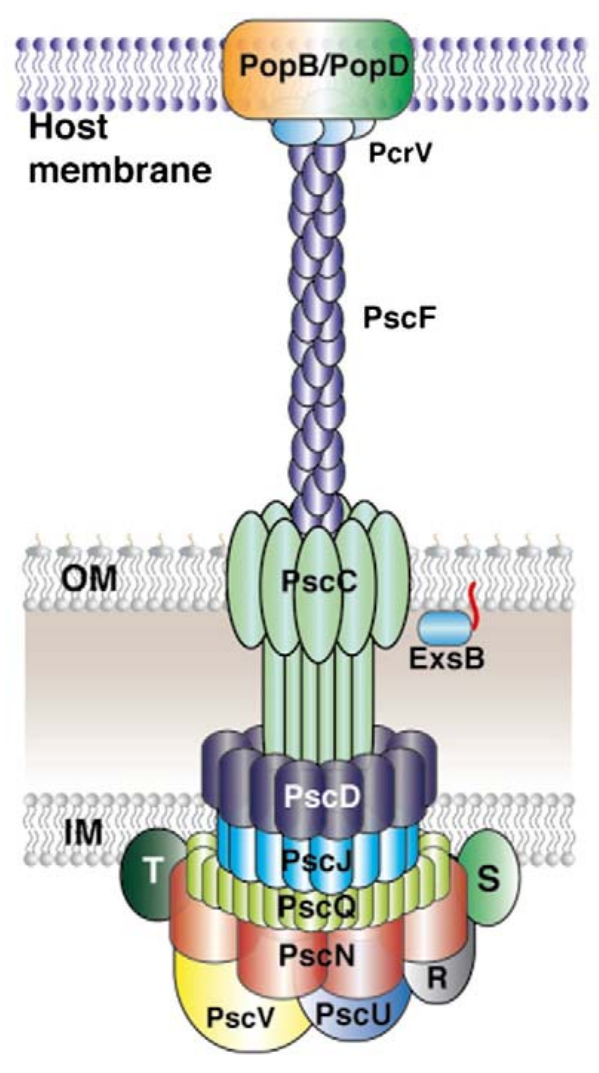

Figure 4. The Pa-T3S structural apparatus. The Pa-T3S injectisome spans the bacterial inner membrane (IM), periplasmic space, and outer membrane (OM). The needle projects from the bacterial outer membrane and is comprised of PscF subunits. PcrV or the V-antigen is located at the tip of the needle structure and serves as an assembly platform for the translocon channel that forms in the host cell membrane (PopB and PopD).

The image appears by permission of Elsevier Limited. The figure is part (a) of a four part figure and was originally published in the article "Structural Characterization and Membrane Localization of ExsB from the Type III Secretion System (T3SS) of Pseudomonas aeruginosa" (2011). Journal of Molecular Biology. 413(1): 236-46 authored by T. Izoré et al. 
Needle-tip complex

LcrV, the needle tip protein in Yersinia, was identified in 1956 as the V-antigen because it induced protection against plague in a mouse model of infection (39). In Pa, the needle tip protein is PcrV, also termed a V-antigen (Fig. 4). While LcrV and PcrV share the highest sequence similarity between needle tip proteins, structural similarity exists with IpaD, BipD, and SipD, the needle tip proteins of Shigella, Burkholderia, and Salmonella, respectively (Table I) $(31,216)$. Needle tip proteins are hydrophilic, and contain two alpha-helices, which can form a coiled-coil, and two globular domains (67). The globular domains of LcrV are larger than those of IpaD, BipD, SipD, and PcrV (reviewed in (215)). In comparison, the needle tip proteins of $E$. coli and Bordetella form filamentous structures at the end of their T3S needles, but these filamentous proteins appear to function in a manner similar to IpaD, BipD, SipD, and PcrV (reviewed in (279). Unlike Yersinia and $\mathrm{Pa}$, the needle tip proteins from Salmonella and Shigella cannot be functionally complemented with each other despite their similarities (238). Together with the needle, needle tip complexes have been implicated in triggering T3S (63, 198, 321).

$\mathrm{Pa}$ PcrV is a 295 amino acid protein that oligomerizes into a ring-like structure at the needle tip $(36,63,111,119,215,216)$. PcrV is encoded by the pcrGVHpopBD operon which in addition to PcrV, encodes for two translocon proteins, PopB and PopD; the chaperone for the translocon proteins, PcrH; and a chaperone/regulatory protein for PcrV, PcrG (Table I) (92, 101, 346). While PcrV has not been isolated from membranes, it is believed to serve as an assembly platform for translocon channel insertion and function in host cell membranes $(28,111,118$, $119,216,283)$. In vitro studies have shown that deregulation of effector secretion occurs with deletion of pcrV, whereby effector proteins are secreted into the extracellular environment in an uncontrolled manner $(119,280,282,303,304)$. This is in contrast to IcrV mutants where Yop secretion is down-regulated $(22,220)$. The functionality of PcrV is tied to the $\alpha 12$ helix in the C- 
terminal domain which is important for intramolecular coiled-coil formation (111). Deletion of the a12 helix, or mutation of residues within this helix, results in normal effector secretion; but functional pore formation and host cell cytotoxicity are prevented $(56,111,119,194)$. Linkerscanning mutagenesis used to further dissect the mechanism underlying the role of PcrV in translocon formation and effector translocation identified: (1) that residues located at the end of the globular N-terminal domain and at the base of the anti-parallel coiled-coil are responsible for assembly and positioning of PcrV on the needle tip; (2) that the region between residues 44 and 64 within the $\mathrm{N}$-terminal globular domain are responsible for secretory control of both translocon and effector proteins; and (3) that the two leucine motifs within PcrV may be responsible for the timing of translocon and effector secretion (280).

Due to the flexibility that has been found in PcrV, it has been proposed that it may undergo a conformational shift which controls effector secretion but not translocon protein secretion (181, 280). In addition, PcrV was shown to regulate effector secretion in combination with its chaperone PcrG in a binding-independent manner (181). In support of these observations, in vitro studies found that ExoS secretion was up-regulated in $\mathrm{Pa}$ strains with an in-frame pcrG deletion (303). Taken together, these studies indicate that PcrV is not only important for translocon insertion and function in host cell membranes, but in combination with PcrG, can also regulate effector translocation into host cells.

\section{Translocon channel}

Effector translocation across the host cell plasma membrane requires two additional proteins, termed translocases. In Pa-T3S, the translocon proteins are PopB and PopD (Fig. 4) (56, 282, 304). PopB and PopD are hydrophobic proteins which form an approximate 2.8-3.5 nm diameter translocon channel in the host cell membrane $(56,119)$. PopB is a $45 \mathrm{kDa}, 392$ amino acid protein that contains two alpha-helical transmembrane domains and two coiled-coil domains. PopD is a $31 \mathrm{kDa}, 296$ amino acid protein with one central transmembrane domain, 
and the C-terminus has the potential to form an amphipathic alpha-helix (283). PcrH, the chaperone for PopB and PopD, is an $18.5 \mathrm{kDa}$ protein that is responsible for the stabilization of both proteins inside the bacterial cytoplasm $(9,35,283)$. Despite PcrH sharing $59 \%$ similarity with LcrH, the chaperone for the Yersinia translocon proteins, YopB and YopD, PcrH has not been shown to be coupled to regulation of Pa-T3S (Table I) $(35,346)$.

Co-immunoprecipitation (CoIP) studies have shown that PopB directly interacts with PopD within red blood cell membranes (119). PcrV serves as an assembly platform for translocon function, by binding to PopD but not PopB. Therefore, the stability of the translocon channel relies on PopB's interaction with PopD in the host cell membrane in conjunction with PopD's interaction with PcrV located on the needle tip. Despite the interaction between PcrV and PopD, PcrV has not been isolated from cell membrane fractions (119).

Deane et al. proposed that upon completion of translocon formation in the host cell membrane, a signal is initiated by a conformational change in the needle tip protein that is propagated down the needle to the bacteria, initiating effector secretion (63). Chaperones, along with $\mathrm{N}$ terminal secretory signals, were identified as key components in preventing premature protein translocation through the T3S needle and translocon channel prior to the bacteria receiving the secretory signal from the needle. Immediately following translation of translocon and effector proteins, chaperones bind to and prevent their target from completing tertiary folding and from being immediately degraded $(103,301)$. Chaperones are also thought to target their proteins to the T3S injectisome, thus preventing T3S proteins from being nonspecifically secreted through the flagellar apparatus (182). Interestingly, another level of secretion regulation by chaperones was recently identified in Salmonella (179). In this new model, chaperones have an essential role in targeting both translocon and effector proteins to a bacterial sorting platform located near the T3S injectisome. Together, the sorting platform and chaperones ensure that the translocon channel proteins are secreted prior to the effector proteins (179). Once the target 
protein is delivered to the sorting platform, the chaperone is removed. It is believed that the T3S proteins travel through the needle and the translocon channel in a partially unfolded state due to the small inner diameter of the needle, and that this process depends on the ATPase located at the cytosolic base of the secretion apparatus $(6,110)$.

While much is known about T3S regulation and structural apparatus assembly, including the size of the translocon channel, what remains unknown is the stoichiometric ratio of PopB to PopD within the channel or what serves as their eukaryotic cell membrane receptor. Another important unknown concept in the translocation process is what is the host cell factor that allows for translocon insertion and function in host cell membranes, as eukaryotic membranes are structured to resist channel formation. By understanding how the eukaryotic cell is involved in translocon formation it may be possible to target these steps to prevent Pa-T3S and the establishment of $P a$ infection.

\section{Effectors}

Following assembly of the T3S structural apparatus, Pa modulates host cell function through the secretion of four known effectors: ExoS, ExoT, ExoU, and ExoY (Table I and Fig. 5). ExoS and ExoT are bi-functional toxins that share $75 \%$ amino acid identity, and contain Rho-GTPase activating protein (GAP) and adenosine-diphosphate (ADP)-ribosyltranserferase (ADPRT) activities $(116,147,166,341)$. ExoU contains phospholipase $A_{2}$ activity that results in a rapid loss of host cell plasma membrane integrity $(85,278)$. ExoY is an adenylate cyclase that contributes to systemic spread of $P a(320)$.

Acute $\mathrm{Pa}$ infections are phenotypically characterized as invasive or cytotoxic due to the mutually exclusive presence of either the exoS or exoU genes $(82,85,88,285)$. ExoS characterizes infections as invasive, is expressed by $58-72 \%$ of clinical $\mathrm{Pa}$ isolates, and is known to function in pathogenesis (reviewed in $(129))(2,297,320)$. ExoU is expressed by 28- 
$42 \%$ of isolates and characterizes infections as cytotoxic (reviewed in $(129))(82,88,285)$. While ExoT is expressed by $92-100 \%$ of $\mathrm{Pa}$ isolates, its contribution to the establishment of $\mathrm{Pa}$ infection remains minor $(82,183,290,320)$. ExoY is found in $89 \%$ of $P a$ clinical isolates (reviewed in (129))(347). 

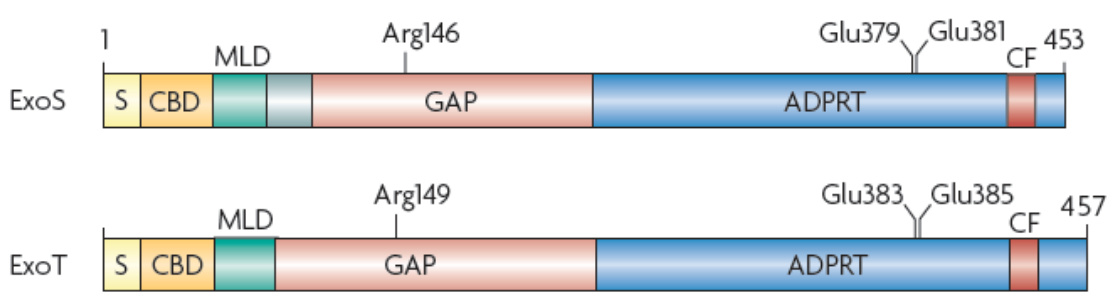

ExoU \begin{tabular}{l|l|l|l|l|}
\multicolumn{7}{c}{ Ser142 Asp344 } & \multicolumn{2}{c}{687} \\
\cline { 2 - 6 } & $\mathrm{S}$ & $\mathrm{CBD}$ & Patatin-like domain (PLA 2 activity) & MLD \\
\hline
\end{tabular}

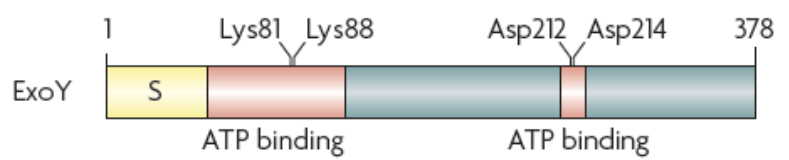

Figure 5. Pa-T3S effector proteins. ExoS and ExoT are bi-functional proteins which contain Rho-GAP (GAP) and ADP-ribosyltransferase (ADPRT) activities. ExoU contains phospholipase activity $\left(\mathrm{PLA}_{2}\right)$ and ExoY is an adenylate cyclase. Each protein contains an $\mathrm{N}$-terminal secretory signal (S). Proteins $S, T$, and $U$ contain a chaperone binding domain (CBD) and a membrane localization domain (MLD). The ADPRT activity of ExoS and ExoT requires binding of the host cell co-factor (CF) 14-3-3 for activation once translocated into the host cell. The key catalytic amino acid residues for each protein are indicated. The presence of exoS or exoU is mutually exclusive in $\mathrm{Pa}$ strains, while exoY is found in $70-95 \%$ of strains and exoT is found in $92-100 \%$ of Pa strains.

This image appears by permission of the Nature Publishing Group. The figure was originally published in the article "The type III secretion system of Pseudomonas aeruginosa: infection by injection" (2009). Nature Reviews Microbiology. 7: 654-665 authored by A.R. Hauser. 


\section{Exoenzyme S}

ExoS was identified in 1978 in the culture supernatant of $\mathrm{Pa}$ strain 388 as an ADPribosyltranserferase, which catalyzes the transfer of ADP-ribose from NAD to eukaryotic host cell proteins $(25,147)$. Skin extracts and sera from burned mice infected with strain 388 contained ExoS highlighting its importance in virulence (25). ExoS is a 453 amino acid, $49 \mathrm{kDa}$ protein and was recognized to be distinct from $\mathrm{Pa}$ ToxA in that it did not ADP-ribosylate elongation factor-2 $(147,219)$. Additional differences between the two ADPRT toxins is that: (1) ToxA is internalized into host cells via receptor mediated endocytosis, and is cleaved by furin once in the host cell cytosol, while ExoS requires the T3S apparatus for delivery into host cells and the host cell protein 14-3-3 for activation; (2) ExoS is heat stable relative to ToxA; and (3) conditions which potentiate ToxA activity partially inactivate ExoS $(46,137,146-148,165,342)$.

Following the identification of $49 \mathrm{kDa}$ ExoS, a $53 \mathrm{kDa}$ form of the ExoS protein was also identified (219). The $53 \mathrm{kDa}$ protein was initially thought to be a pro-enzyme form of ExoS that would then be cleaved to the $49 \mathrm{kDa}$ enzymatically active form $(172,219)$. This hypothesis was supported by evidence that the $53 \mathrm{kDa}$ form was found to contain $0.2 \%$ of the ADPRT activity of the $49 \mathrm{kDa}$ protein $(341,346)$. Through genetic analysis, it was later recognized that the $53 \mathrm{kDa}$ protein contained $75 \%$ amino acid similarity to the $49 \mathrm{kDa}$ protein, and was encoded by a different gene, termed exoT $(341,346)$. Thus, the $53 \mathrm{kDa}$ protein initially believed to be a less active pro-enzyme form of ExoS was recognized to be a distinctly different protein, ExoT. Like ExoS, ExoT was further characterized as a bi-functional toxin possessing both Rho-GAP and ADPRT activity. ExoS was subsequently discovered to be secreted by a T3S system when genetic analysis showed similarity of $\mathrm{Pa}$ genes with genes from the Yersinia Ysc T3S system $(101,131,343,346)$. 
Enzymatic activity

The ExoS protein contains several domains, including two catalytic domains. The amino terminal of ExoS contains a secretory signal followed by a chaperone binding domain (Fig. 5) (20). A membrane localization domain (MLD) is located between residues 51-72, and targets ExoS to the eukaryotic plasma membrane where it is essential for ADP-ribosylation of host cell substrates, such as Ras (Fig. 5) (232, 351). The MLD is also important for Rho-GAP activity, where, as studies have shown, mutation of the MLD abolishes ExoS GAP activity in vivo (353).

The ExoS GAP domain spans residues 96-233 and contains an arginine finger at residue 146 that is responsible for down-regulation of host cell Rho-family proteins (Fig. 5) $(116,167)$. The GAP domain of ExoS is alpha-helical, and while there does not appear to be any structural similarity between ExoS GAP and eukaryotic Rho-GAPs, a low level of similarity is shared with GAP domains found at the amino terminals of Yersinia YopE and Pa ExoT (154, 166, 339, 345). Within eukaryotic cells, ExoS GAP activity towards Rac1 and Cdc42 results in actin reorganization, while inactivation of Rho stimulates actin stress fiber formation (116, 136, 167, 233). Interestingly, kinetic analysis has shown that ExoS contains higher catalytic activity towards Rho-family proteins than the eukaryotic p50RhoGAP protein (186). In terms of ExoS catalytic activity towards individual Rho-family proteins, ExoS exhibits highest activity towards Rac1 and Cdc42, followed by RhoA (186). Modulation of host cell actin dynamics by ExoS GAP activity serves as an anti-internalization factor to prevent bacterial uptake and $\mathrm{Pa}$ death (52, $100,108,116,263)$.

ExoS ADPRT activity targets the Ras family of low molecular weight G (LMWG) proteins where ADP-ribosylation prevents their interaction with downstream targets. This results in cell rounding and inhibition of DNA synthesis $(95,96,106,135,205,326)$. Cyclophilin A and the ezrin/radixin/moesin (ERM) family of proteins have also been identified as additional ExoS ADPRT substrates $(69,195,196)$. The ExoS ADPRT domain spans residues $234-453$ and 
requires the host cell factor 14-3-3 for catalytic activity (Fig. 5) (46, 102, 137, 163). Following translocation into the host cell, ExoS binds to 14-3-3 between residues 420 and 429 located within the ADPRT domain. Additionally, ExoS binding to 14-3-3 depends on leucine residues 422, 423, 426, and 428 that are located within an amphipathic groove of 14-3-3 $(134,226)$. Contrary to other 14-3-3 binding motifs, crystal structure analysis has shown that ExoS binds to 14-3-3 in a phosphorylation independent manner, with reversed orientation via hydrophobic interactions (226).

ADP-ribosylation of host cell proteins by ExoS depends on catalytic glutamic acid residues 379 and 381. In vitro, E379 participates in the transfer of ADP-ribose to target substrates and E381 contributes to catalytic activity of ExoS $(188,249)$. Through cell line analysis, a hierarchy of eukaryotic substrate modification by ExoS ADPRT activity has been identified. In human cell lines, Ras and RalA were identified as early substrates followed by Rab5, Rab8, Rab11, and Rab7. Rac1 and Cdc42 were found to be late substrates (97). However, in rodent cell lines Rac1, Rab5, and Rab8 are not ExoS ADPRT substrates (270). These results indicate that the host cell type influences the differences in ExoS toxicity.

Role of ExoS in infection

ExoU is currently believed to be the most virulent of the Pa-T3S effectors because it is 100 times more cytotoxic than ExoS $(73,183,290,322)$. The mutually exclusive presence of either ExoU or ExoS in $\mathrm{Pa}$ strains, taken together with results from clinical studies, has shown that ExoS may still have a prominent role in infection. Early clinical studies of $P a$ isolates from tracheal, urinary tract, or wound infections identified significantly higher levels of ExoS from wound and urinary tract sites, and that isolates producing increased ExoS could be isolated from both colonizing and infection sites $(124,272)$. Together, these studies led to the hypothesis that in vivo wound or urinary tract conditions may allow for increased ExoS production, and that this could be enhanced by prolonged infection (124). 
The majority of studies investigating the role of both ExoS and the Pa-T3S system in $\mathrm{Pa}$ infection have been conducted in patients with respiratory disease. In studies of patients with various lower respiratory infections, 30 of $108(28 \%) \mathrm{Pa}$ isolates were found to express ExoS (269). The mortality rate for patients infected with a PcrV negative $\mathrm{Pa}$ isolate was $3 \%$, which increased to $25 \%$ when the $\mathrm{Pa}$ isolate also expressed ExoS or ExoU, thus highlighting the role of both ExoS and ExoU in disease severity (269). Studies of $\mathrm{Pa}$ isolates from patients with VAP found that 23 of 35 isolates (66\%) contained the exoS gene, 16 of which produced detectable amounts of ExoS when analyzed in vitro (130). Twelve of the 16 ExoS secreting strains were isolated from patients with severe disease, further suggesting that ExoS contributes to disease severity (130). Finally, studies by Zhuo et al. found the presence of the type III proteins ExoS, ExoU, and PcrV in: (1) 16 of $24(66.7 \%) \mathrm{Pa}$ isolates from patients with respiratory infections with low Pa burden; (2) 13 of 17 (76.5\%) patients with high Pa burden without VAP; and (3) 17 of $28(60.7 \%)$ patients with high Pa burden who developed VAP (356). Specifically, ExoS has been found in $63 \%$ of isolates from patients with low $\mathrm{Pa}$ burden, $54 \%$ in isolates from patients with high $\mathrm{Pa}$ burden without VAP, and in $47 \%$ of isolates with high $\mathrm{Pa}$ burden and VAP (282). From these studies it was suggested that $P a$ are more likely to be planktonic and not contained within biofilm in patients with a high $\mathrm{Pa}$ burden, and that these $\mathrm{Pa}$ isolates are more virulent due to the high percentage that express components of the T3S (356).

The identification of ExoT by Yahr et al. lends support to an increased pathogenicity of $\mathrm{Pa}$ infections associated with co-expression of ExoS or ExoU with ExoT (73, 269, 341). A recently published study by El-Solh et al. highlights the importance of Pa-T3S and the effectors ExoS, ExoU, and ExoT in $\mathrm{Pa}$ bloodstream infections (73). Of 85 patients diagnosed with a $\mathrm{Pa}$ bloodstream infection, 37 (44\%) patients had an isolate that expressed at least one of the type III secretory proteins. The ExoU/ExoT phenotype was identified as the most prevalent in 18 of $37(49 \%)$ isolates, while the ExoS/ExoT phenotype was identified in eight of $37(22 \%)$ isolates. 
Two (5\%) isolates were found to express ExoS alone, and nine (24\%) expressed ExoT alone (73). Previous antimicrobial treatment was found in 48 patients, $65 \%$ of which had a T3S positive phenotype. Of these, ExoS was identified in five $(23 \%)$ isolates resistant to ciprofloxacin and in one $(7 \%)$ isolate resistant to gentamicin (73). Together these studies suggest that despite antimicrobial treatment, patients who had T3S positive bloodstream isolates had a poorer clinical prognosis. The question from these studies is why is expression of ExoT with ExoS or ExoU important to infection? ExoT, like ExoS, contains Rho-GAP activity that functions in preventing internalization $(52,108,166)$. In strains producing ExoS alone, a low percentage of $\mathrm{Pa}$ can be internalized $(76,88,243)$. ExoU does not contain any known antiinternalization properties. Therefore, it may be possible that the role of ExoT expression in conjunction with ExoS or ExoU is to enhance the anti-internalization capabilities of $\mathrm{Pa}$, especially since ExoT is expressed by nearly all strains $(183,290,320)$.

The role of T3S in acute $\mathrm{Pa}$ infections is supported by clinical results showing that mortality rates are increased in patients with $\mathrm{Pa}$ isolates expressing T3S proteins and that antibodies against T3S components confer protection from Pa-T3S infection. Additionally, the role of ExoS in infections is supported by clinical results showing that mortality is increased in patients colonized with ExoS or ExoU expressing $\mathrm{Pa}$ isolates compared to PcrV only expressing isolates.

The current model for the role of T3S in establishment of $\mathrm{Pa}$ infection is shown in Figure 6. In this model, $\mathrm{Pa}$ expressing flagella and pili bind to the host cell surface, potentially through asialo-GM1, an asialylated glycolipid located within epithelial cell membranes or through adhesion to the CFTR $(81,113,122)$. Disruption of epithelial integrity from components of the T3S system, such as ExoS or ExoU, followed by disruption of the ECM by Pa factors such as elastase and proteases, allows for transversal of $\mathrm{Pa}$ to the blood stream to facilitate bacterial dissemination to other sites of the body $(129,218,231,297)$. In the establishment of a chronic 
infection, following bacterial binding and initiation of T3S, Pa will form a biofilm on the epithelial surface (reviewed in (334)). Production of alginate in conjunction with down-regulation of flagella, pili, LPS, and T3S by $\mathrm{Pa}$ reduces immune recognition of $\mathrm{Pa}$ and contributes to persistence of the bacteria $(40,125,192)$. The step which remains the least understood in this model of $\mathrm{Pa}$ infection is what is the host cell factor that allows for T3S translocon insertion and function in the host cell membrane. 


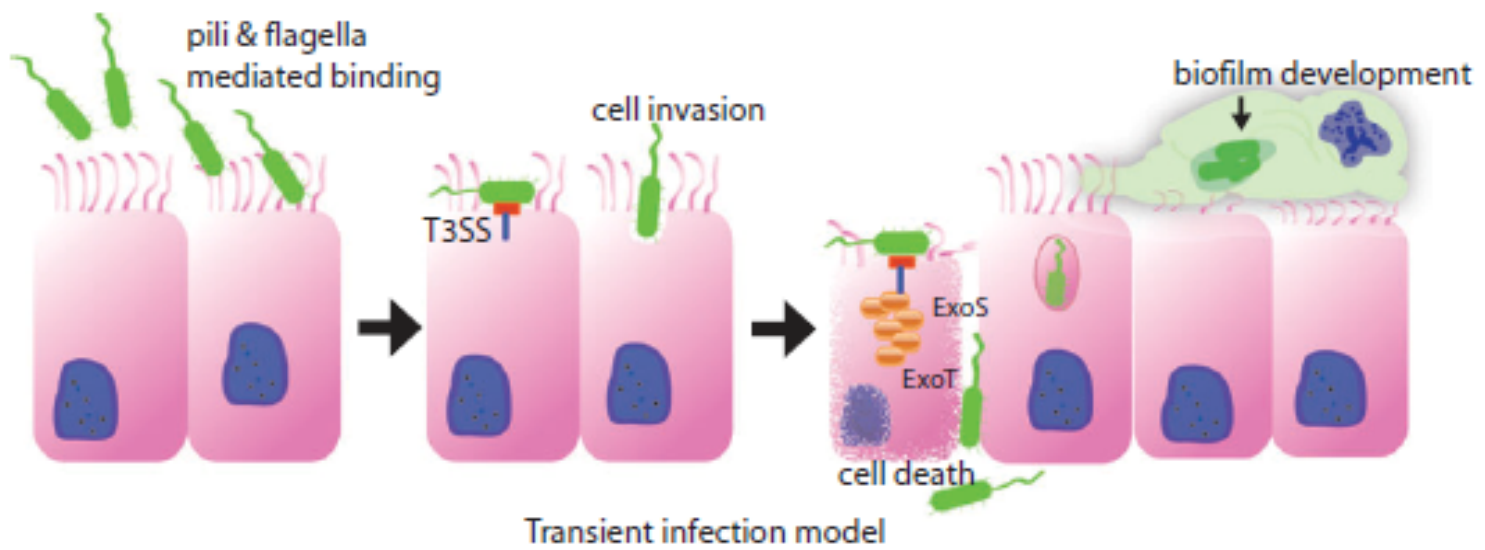

Figure 6. Model of Pa-T3S in the establishment of $\mathrm{Pa}$ infection. $\mathrm{Pa}$ binds to the host epithelial layer via flagella and pili. Toxin production, presumably due to the T3S system, results in the damage to the epithelial barrier which allows traversal of the bacteria to the blood stream. Bacteria that remain at the initial site of infection may go on to produce a biofilm.

The image appears by permission of John Wiley and Sons. This figure was part (a) of a two part figure originally published in the review "Pseudomonas aeruginosa: Host defence in the lung diseases" (2010). Respirology. 15(7):1037-1056 authored by Williams et al. 


\section{Host Cell Influences in Pseudomonas-T3S}

Unlike obligate pathogens which target specific cell types, $P a$ is able to cause disease in a wide variety of cell and tissue types. While it is: (1) hypothesized that host cell factors at the site of infection may influence the severity of disease, as indicated by increased ExoS production in wound and UTI Pa isolates; (2) known that T3S is involved in the establishment of infection; and (3) known that establishment of acute cellular $\mathrm{Pa}$ infections parallels cellular sensitivity to Pa-T3S; it is unknown what the host cell factor is that allows for the establishment of infection in so many tissue and cell types (reviewed in(129))(33, 124, 206, 271). Asialo-GM1, a receptor for flagella, pili, and LPS, the CFTR, and epithelial cell polarity were factors implicated in Pa-T3S, but no clear role of the host cell had been elucidated $(62,87,156,243)$.

The first sets of studies developed in this area aimed to identify the host cell factors responsible for the insertion and function of Pa-T3S translocon proteins in host cell membranes. Initially, purified $\mathrm{Pa}$ translocon proteins, $\mathrm{PopB}$ and $\mathrm{PopD}$, were found to bind to liposomes in a cholesterol-independent manner, but oligomerized and induced channel formation in the liposome in a cholesterol-dependent manner (283). In 2005, Hayward et al. showed that translocon proteins, SipB and IpaB, from Salmonella and Shigella respectively, bound to cholesterol in vitro with high affinity and that depletion of eukaryotic cell membranes of cholesterol blocked T3S (132). However, Faudry et al. subsequently showed that channel formation in liposomes was cholesterol independent and that both PopB and PopD bound to phosphotidylserine (80). Together, the role of cholesterol in Pa-T3S remained unclear due to differences in the experimental systems used. At the same time, studies evaluating over 40 cell lines including epithelial, endothelial, fibroblasts, macrophages, and T cells showed that only two types of cells were resistant to Pa-T3S, confluent polarized epithelial cells and promyelocytic HL-60 cells $(206,271)$. Both cell culture models could be induced to become sensitive to Pa-T3S through disruption of apical-basal lateral polarity for confluent monolayers 
or differentiation of HL-60 cells with phorbol esters $(206,271)$. Studies have also found that translocon channel formation in host cell membranes can be influenced by translocated effectors. Studies in Yersinia have shown that the effectors YopE, YopT, and YopK can inhibit T3S channel formation in host cell membranes, which contributes to a dampening of host inflammatory response towards the bacteria $(34,68,324)$. Additionally, studies where Pa-T3S effectors were expressed in Yersinia have indicated that $\mathrm{Pa}$ may have the ability to regulate translocon formation and effector translocation via its effectors, but it remains to be determined if other T3S systems have this capability (4).

\section{Present Studies}

Knowing that a cellular or immunocompromise is needed for the establishment of $P a$ infection and $\mathrm{Pa}$-T3S, the purpose of the first set of studies presented in this dissertation was to identify the host cell factors that allow for the establishment of Pa-T3S infection. Two cell models were utilized to determine what would induce resistance to Pa-T3S in a sensitive line (HT-29 cell model) and what would induce sensitivity in a resistant cell line (HL-60 cell model). In using the T3S effector ExoS to monitor sensitivity or resistance to Pa-T3S infection, these studies identified that sensitivity to Pa-T3S was induced with acquisition of cell migration, while resistance was induced when eukaryotic cells lost cell migration capabilities. Specifically, it was identified that Rac1/Cdc42-mediated focal complexes, which are located at the leading edge in migrating cells, were important in T3S sensitivity. From these studies, we developed the hypothesis that as cells migrate, actin-induced changes in the host cell alters the membrane in a manner that allows for translocon insertion and effector translocation, and that this process is mediated by Rac1 and Cdc42.

The second set of studies was designed to further investigate the hypothesis that the leading edge in migrating cells is the host cell factor required for Pa-T3S induction. A T24 cell model 
was used for these studies because they exhibit a highly migratory phenotype where the leading edge can be differentiated from the trailing edge. In order to understand how ExoS might be regulating translocation at the leading edge, we utilized $P$ a strains expressing ExoS with mutations that abolished its GAP (R146A) and/or ADPRT (E379A, E381A, or E379A/E381A) activities. These studies found: (1) Pa expressing WT ExoS localized to the leading edge in migrating T24 cells. This localization was significantly increased compared to T24 cells treated with $\mathrm{Pa}$ expressing a pUCP empty vector or translocon mutant $(\triangle \mathrm{PopB})$. (2) Increases in ExoS translocation by Pa expressing ExoS deficient in GAP activity were attributed to continued effector translocation by internalized $\mathrm{Pa}$. (3) Increases in ExoS translocation by $\mathrm{Pa}$ expressing ExoS deficient in ADPRT activity related to maintenance of leading edge properties. (4) Increases in ExoS translocation by ExoS deficient in both GAP and ADPRT activities related to increased bacterial binding, $\mathrm{Pa}$ internalization, continued effector translocation within host cells, and maintenance of the T24 leading edge actin structure. (5) Lastly, both Pa and ExoS were observed to align along actin filaments. From these studies the hypothesis evolved into actin induced changes in membrane dynamics at the leading edge of migrating cells allows for translocon insertion. ExoS translocation into the host cell is facilitated by actin filament linkage to the membrane via ERM proteins. As ExoS tracts along actin and reaches its targets within the host cell, it subsequently down-regulates effector translocation through disruption of the actin linkage to the membrane by interfering with ERM proteins, and preventing $\mathrm{Pa}$ internalization via inactivation of Rho-family GTPases.

The final set of studies presented in this dissertation utilized cell models which are known to differ in regulation of leading edge dynamics. MTC and MTLn3 cells are closely related cell lines used to study differences in host cell metastasis. MTC cells were isolated from a mammary tumor and are a non-metastatic epithelial cell line. MTLn3 cells are from the same mammary tumor, but had metastasized to the lung, where they were isolated. Previous studies 
have shown that MTC cells regulate leading edge dynamics via Rac1 while MTLn3 cells regulate leading edge dynamics via Rho $(71,72)$. Studies presented in this chapter found that increased sensitivity of MTLn3 cells, compared to MTC cells, was attributed to increased $\mathrm{Pa}$ internalization by MTLn3 cells. Treatment of MTLn3 cells with the ROCK inhibitor Y-27632 was found to decrease sensitivity to Pa-T3S to levels comparable to MTC cells, while treatment of MTC cells with the Rac1 inhibitor, NSC23766, was found to increase MTC cell sensitivity to PaT3S. From these studies the hypothesis developed that increased MTLn3 sensitivity to Pa-T3S occurs because these cells are resistant to ExoS GAP activity due to high levels of Rho at the leading edge, while MTC cells are sensitive to ExoS GAP activity due to high levels of Rac1 at the leading edge. Studies using a third cell line, Madin-Darby canine kidney (MDCK) cells, to analyze wounded monolayer sensitivity to Pa-T3S found that wounds were most sensitive to Pa-T3S 4-6 hr post-wounding. In addition, sensitivity was due to $P a$ internalization, which can potentially be attributed to the increased presence of Rho at the leading edge in the cells migrating to close the wound.

Together, the studies presented in this dissertation highlight the mechanism underlying the opportunistic nature of $\mathrm{Pa}$ infections, which are known to target wounded epithelial barriers and support the hypothesis that host cell migration properties are contributing factors in $\mathrm{Pa}-\mathrm{T} 3 \mathrm{~S}$ translocation and in the establishment of $\mathrm{Pa}$ infection. Specifically, at the leading edge, Rac1 and Cdc42-mediated actin polymerization induces changes in membrane dynamics which allows for translocon insertion. Pa then utilizes actin linked to the plasma membrane via phosphorylated ERMs to translocate ExoS into the host cell. In turn, ExoS regulates $\mathrm{Pa}-\mathrm{T} 3 \mathrm{~S}$ by targeting Rho-family GTPases to prevent $\mathrm{Pa}$ internalization, and by potentially targeting ERM proteins to interrupt the actin-plasma membrane linkage, which prevents further translocation. Increased T3S sensitivity of certain cancer cell lines, as well as cell lines which migrate via Rho-mediated amoeboid migration can be attributed to the cells being resistant to ExoS GAP 
activity, whereby $\mathrm{Pa}$ can be internalized and continue to translocate effectors within the host cell (Fig. 7). 
Figure 7. Model of the role of the host cell in the establishment of Pa-T3S infection. A) In Rac1-mediated cell migration, eukaryotic cells migrate via a mesenchymal phenotype. Pa preferentially targets the leading edge of these migrating cells due to active actin polymerization and changes in membrane viscosity, which allow for translocon insertion. ExoS is translocated into the host cell via actin at the leading edge. Once inside the cell, ExoS GAP activity targets GTP-bound Rac1 and causes hydrolysis to GDP-bound Rac1 to prevent Pa internalization by the host cell. ExoS ADPRT activity interrupts actin-plasma membrane linkage through disruption of ERM proteins, which regulates T3S by inhibiting further ExoS translocation. B) In Rho-mediated cell migration, eukaryotic cells migrate via an amoeboid phenotype. GTP-bound Rho is more resistant to the effects of ExoS GAP activity and as a result, $\mathrm{Pa}$ can use actin to become internalized in membrane vesicles and continue T3S translocation within the host cell. ExoS ADPRT has limited inhibitory effects on pERM mediated actin-plasma membrane linkage and as a result, ExoS translocation does not appear to be limited. 
A

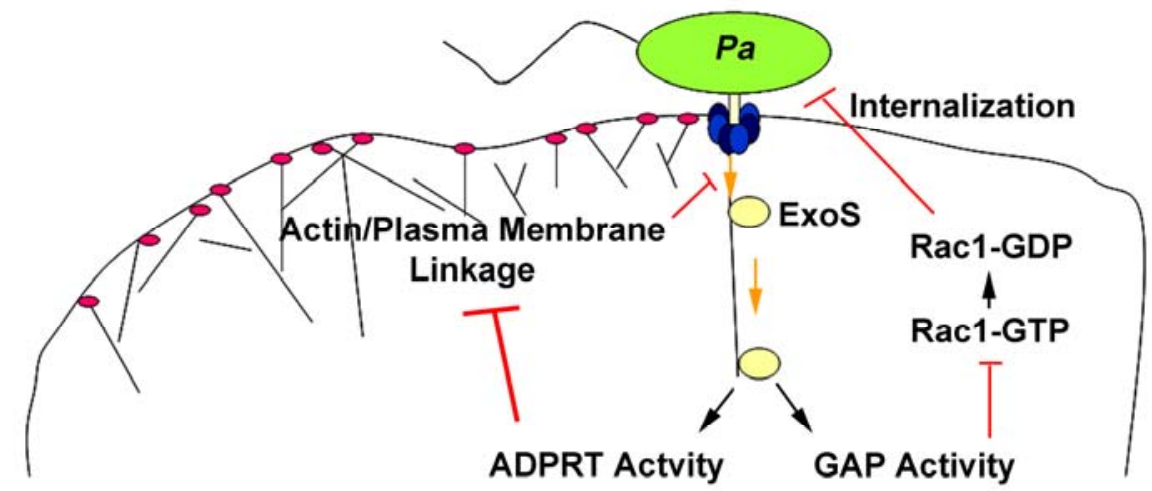

B

Rho-Mediated Cell Migration

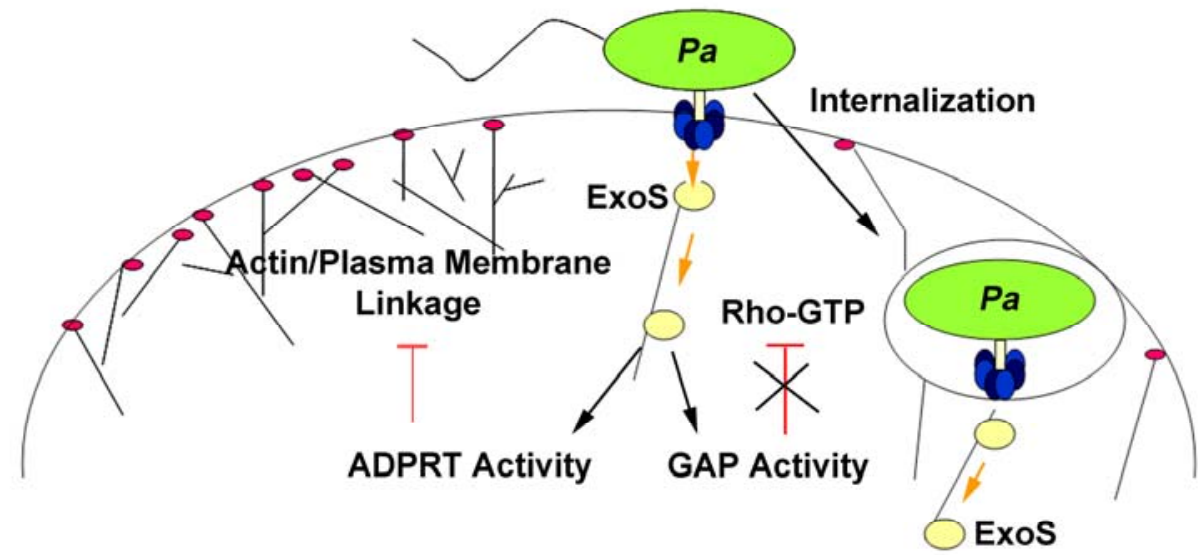

$$
\begin{aligned}
& \text { pERM } \\
& \text { C Pa-T3S System } \\
& / \text { Actin }
\end{aligned}
$$




\section{Chapter 2: Role of Host Cell Polarity and Leading Edge Properties in Pseudomonas aeruginosa Type III Secretion}

Contents of this chapter were published in February of 2010 in Volume 156, pages 356-373 of Microbiology and appear with permission from the Society for General Microbiology.

The authors on the manuscript are: Dacie R. Bridge, Matthew J. Novotny, Elizabeth R. Moore, and Joan C. Olson

Dacie R. Bridge and Matthew J. Novotny are co-first authors 


\section{SUMMARY}

Type III secretion (T3S) functions in establishing infections in a large number of Gram-negative bacteria, yet little is known about how host cell properties might function in this process. We used the opportunistic pathogen, Pseudomonas aeruginosa, and the ability to alter host cell sensitivity to Pseudomonas T3S to explore this problem. HT-29 epithelial cells were used to study cellular changes associated with loss of T3S sensitivity, which could be induced by treatment with methyl-beta-cyclodextrin or perfringolysin O. HL-60 promyelocytic cells are innately resistant to Pseudomonas T3S and were used to study cellular changes occurring in response to induction of T3S sensitivity, which occurred following treatment with phorbol esters. Using both cell models, a positive correlation was observed between eukaryotic cell adherence to tissue culture wells and T3S sensitivity. In examining the type of adhesion process linked to T3S sensitivity in HT-29 cells, a hierarchal order of protein involvement was identified that paralleled the architecture of leading edge (LE) focal complexes. Conversely, in HL-60 cells induction of T3S sensitivity coincided with the onset of LE properties and the development of actin-rich projections associated with polarized cell migration. When LE architecture was examined by immunofluorescent staining for actin, Rac1, IQ-motif-containing GTPase-activating protein 1 (IQGAP1) and phosphatidylinositol 3 kinase (PI3 kinase), intact LE structure was found to closely correlate with host cell sensitivity to $P$. aeruginosa T3S. Our model for host cell involvement in Pseudomonas T3S proposes that cortical actin polymerization at the LE alters membrane properties to favor T3S translocon function and the establishment of infections, which is consistent with Pseudomonas infections targeting wounded epithelial barriers undergoing cell migration. 


\section{INTRODUCTION}

Originally identified because of its role in Yersinia virulence (50), type III secretion (T3S) is now recognized to contribute to the pathogenesis of a large number of Gram-negative bacteria. T3S allows the direct translocation of 'effectors' from the bacterial cytosol into eukaryotic cells, enabling bacteria to manipulate host cells to establish infections while evading immune responses. The T3S system includes a bacterially formed 'injectisome' needle-like nanostructure that serves as a conduit for transferring bacterial effectors to eukaryotic cells. A bacterially formed 'translocon' channel is then believed to mediate effector translocation across host cell membranes. The mechanism underlying T3S translocon channel formation and host involvement in this process remains the least understood events in T3S. We have used the opportunistic pathogen, Pseudomonas aeruginosa, and insights into T3S provided by it to study this problem.

$P$. aeruginosa is a ubiquitous environmental bacterium that is capable of causing infections of high morbidity and mortality in individuals compromised by wounds, immune defects, or cystic fibrosis. While the cellular alteration predisposing individuals to $P$. aeruginosa infections is not known, factors that have been implicated include: alterations in expression of putative $P$. aeruginosa receptors, such as asialo-GM1, alterations in the cystic fibrosis transmembrane conductance regulator, and cellular changes affecting epithelial cell polarity $(62,91,156,243)$. Studies have also drawn attention to the role of T3S during initial phases of $P$. aeruginosa infections $(56,117,151)$. Consistent with this notion, cellular properties influencing $P$. aeruginosa infections, such as apical-basolateral polarity $(91,156,243)$, also influence sensitivity to $P$. aeruginosa T3S (Pa-T3S) (206). The integral role of T3S in the establishment of $P$. aeruginosa infections is evident in the ability of the T3S translocon protein, PcrV, to induce adaptive immune protection against $P$. aeruginosa infections (282). $P$. aeruginosa offers an advantage in studying mechanisms underlying T3S translocon function and host cell 
involvement in this process for, as an opportunistic pathogen, eukaryotic cells sensitive or resistant to Pa-T3S have been identified and are available for mechanistic comparisons (206).

Current studies support the suggestion that in Pa-T3S translocon formation, the needle tip protein, PcrV oligomerizes into a ring-like structure in association with the T3S needle complex and serves as an assembly platform for membrane insertion and pore formation by hydrophobic translocator proteins, PopB and PopD $(111,119,216)$. Consistent with this model, PcrV is required for T3S pore formation in red blood cells and for translocation of effectors into nucleated cells (118). Also consistent with this model, PopB and PopD maintain intrinsic pore forming properties, and PopB and PopD, but not PcrV, can penetrate into and be detected in eukaryotic cell membrane fractions $(80,283)$. A precise understanding of the mechanism of T3S translocon formation and function, however, has been hampered by an inability to detect the direct interaction of PcrV with either PopD or PopB $(9,119,283)$. A role of host cell membrane properties in Pa-T3S translocon function has been revealed by studies that found PcrV to be required for T3S pore formation in eukaryotic cells, whereas PcrV was not required for pore formation in lipid vesicles $(119,216,283)$.

The limited understanding of host cell involvement in T3S translocon function attests to experimental difficulties in identifying bacterial-host cell interactions during T3S. Current studies have implicated host cell lipid rafts and their cholesterol component in T3S translocon formation, but the mechanism underlying their role in T3S is unclear $(132,175,176,259,318)$. In our analyses of over 40 cell lines (including epithelial, fibroblasts, endothelial, macrophage, and T-cells), two cell types were identified to be resistant to T3S: confluent polarized epithelial cells and promyelocytic HL-60 cells $(206,271)$. We also found host cell sensitivity to Pa-T3S to be an alterable property. Polarized epithelial cells can become T3S sensitive by disruption of tight junctions and loss of apical-basolateral polarity (206). HL-60 cells can be converted to PaT3S-sensitive by treatment with the differentiating phorbol ester, 12-O-tetradeconylphorbol-13- 
acetate (TPA) (271). In studies of HL-60 cells, resistance to Pa-T3S has been found to occur at the level of membrane translocation.

The questions being asked in this study are: (1) how do eukaryotic cells change in conjunction with alterations in Pa-T3S sensitivity; and (2) how can this be applied to understanding the role of host cell properties in T3S translocon function? Two basic approaches were used to answer these questions. First, Pa-T3S-sensitive HT-29 epithelial cells were used as a model to examine alterations in cellular properties occurring in conjunction with loss of host cell sensitivity to Pa-T3S. Second, Pa-T3S-resistant HL-60 cells were used to examine alterations in cellular properties that occur upon induction of $\mathrm{Pa}$-T3S sensitivity. Both approaches led to the recognition of a relationship between host cell polarity, directional migration, and leading edge (LE) properties with Pa-T3S sensitivity. These findings have led us to hypothesize that as an opportunistic pathogen $P$. aeruginosa relies on alterations in membrane properties at the LE for Pa-T3S translocon insertion and function. 


\section{METHODS}

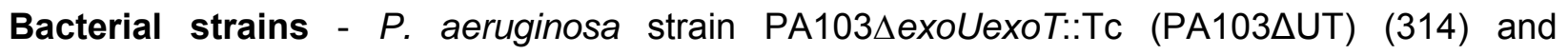
PA103 $\triangle \mathrm{PopB}$, a T3S translocon mutant strain, were provided by Dara Frank (Medical College of Wisconsin, Milwaukee, WI, USA). Hemagglutinin (HA)-tagged ExoS (ExoS-HA) (232) was provided by Joseph Barbieri (Medical College of Wisconsin). PA103 $\Delta$ UT expressing plasmidencoded ExoS-HA (Pa-ExoS-HA) was the primary strain used in this study to assay for host cell sensitivity to Pa-T3S. PA103AUT expressing a plasmid encoded enzymatically inactive ExoSGAP/ADPRT mutant (having R146A-GTPase activating protein and E379A/E381A-ADPribosyltransferase mutations) was used as a control to evaluate the role of ExoS enzymatic function in our analyses and was constructed as previously described (95).

Eukaryotic cell culture - HT-29, T24, and HL-60 cells were obtained from American Type Cell Culture Collection (ATCC, Manassas, VA) and were cultured according to ATCC specifications in media supplemented with $10 \%$ fetal bovine serum, $100 \mathrm{U}$ penicillin $\mathrm{ml}^{-1}$, and $100 \mu \mathrm{g}$ streptomycin $\mathrm{ml}^{-1}$ (Hyclone).

Bacterial-eukaryotic cell co-culture studies and drug treatments - In co-culture studies of T3S-sensitive cells, HT-29 or T24 cells were seeded at $2.5 \times 10^{5}$ or $1.0 \times 10^{5}$ cells ml $^{-1}$, respectively, unless otherwise specified, and grown to 70-90\% confluency. Both HT-29 and T24 cells were examined using the drug treatments listed in Supplementary Table S1 to confirm that drug effects were not cell line specific. Prior to drug treatment, cells were washed twice with Dulbecco's PBS (DPBS; HyClone), and drugs were added in cell line specific medium containing $0.6 \%$ BSA (bovine serum albumin, Sigma-Aldrich). Cells were treated with drugs as specified and then co-cultured with $10^{7}$ c.f.u. $\mathrm{ml}^{-1}$ of the indicated $P$. aeruginosa strain or no bacteria for 4-5 h, as previously described (224). In studies using perfringolysin-O (PFO, C459A; (291)), PFO was activated with $5 \mathrm{mM}$ DTT. Monomer locked PFO (ssPFO, 
T319C/V334C; (250)) and prepore locked PFO (dsPFO, S190C/G57C; (144)) were dialyzed against HEPES buffered saline prior to addition to cells. All PFO constructs were provided by Rodney Tweten (University of Oklahoma Health Sciences Center, Oklahoma City, OK, USA).

In co-culture studies of T3S resistant cells, HL-60 cells were seeded at a density of $1 \times 10^{5}$ cells $\mathrm{ml}^{-1}, 12 \mathrm{~h}$ prior to TPA differentiation or co-culture with $P$. aeruginosa. Terminal differentiation was induced with $20 \mathrm{nM}$ TPA, (Calbiochem) $(47,184)$ for the indicated times. Prior to co-culture, non-adherent HL-60 cells were collected, centrifuged, and reseeded with the corresponding adherent fractions (when present) in medium containing $0.6 \%$ BSA. Cells were co-cultured for 4-5 h with Pa-ExoS-HA (m.o.i. 30:1).

Analysis of eukaryotic cell cytotoxicity, adherence, and Pa-T3S sensitivity - Following coculture of HT-29 cells with $P$. aeruginosa strains, bacteria were removed, cells detached, and extracellular proteins degraded with $0.25 \%$ trypsin-1mM EDTA (Hyclone). Cells were washed two times in medium containing $200 \mu \mathrm{g}$ ciprofloxacin $\mathrm{ml}^{-1}$, and an aliquot of cells was examined for cell viability, adherent cells, and total cell number using trypan blue staining. The percentage of adherent HT-29 cells was calculated relative to non-drug treated control adherent cells remaining in tissue culture wells following co-culture studies. A second aliquot of cells was examined for total protein (BCA Protein Assay; Pierce). The remainder of cells was processed for either cell extract or membrane fractionation analysis. For cell extract analysis, cells were resuspended and lysed in $4 \mathrm{X}$ Laemmli sample buffer (174). For cell membrane analysis, cells were resuspended in digitonin fractionation buffer and fractionated as previously described (264). RalA localization to the membrane fraction was used to confirm the specificity of the fractionation procedure.

Following studies of co-culture of HL-60 cells with $P$. aeruginosa strains, cells were collected and washed with medium containing ciprofloxacin, extracellular proteins were degraded with 
trypsin and cell membrane fractionation was performed as described for HT-29 cells. To quantify HL-60 cell adherence to tissue culture wells relative to Pa-T3S sensitivity, HL-60 cells were seeded in duplicate, treated with TPA for increasing times, and then co-cultured with $\mathrm{Pa}$ ExoS-HA. One sample was assayed for T3S effector translocation, as described below. The other sample was assayed for the percentage of adherent cells by enumerating cells adherent and non-adherent to tissue culture wells in each sample using trypan blue staining; the percentage of adherent cells was calculated relative to total cells.

Eukaryotic cell sensitivity to Pa-T3S was assayed based on the translocation of ExoS-HA, ExoS ADP ribosylation of RalA, or membrane insertion of translocon proteins, $\mathrm{PopB}$ and $\mathrm{PopD}$, as determined by SDS-PAGE and immunoblot analysis (95, 96, 271). Comparisons of host cell T3S sensitivity were based on equal protein loading, and detection antibodies included: monoclonal anti-HA to detect ExoS-HA (Covance Research Products); monoclonal anti-RalA to detect ExoS effector function (BD-Transduction Laboratories); and polyclonal anti-PopB and anti-PopD to detect translocon membrane insertion (provided by Joseph Barbieri and Dara Frank). Monoclonal anti-glyceraldehyde 3-phosphate dehydrogenase (GAPDH; Chemicon International) was used as a protein expression/loading control. Immunoblots were developed using horseradish peroxidase (HRP)-conjugated anti-mouse or anti-rabbit IgG (Sigma-Aldrich) and detected by enhanced chemiluminescence (GE Healthcare Bio-Sciences Corp). Percentage RalA ADP ribosylation was quantified relative to total RalA based on densitometry analyses using ImageJ $1.40 \mathrm{~g}$ software (Wayne Rasband, $\mathrm{NIH}$ ), averaging a minimum of three independent experiments.

Analysis of effects of drug treatments on bacteria - Effects of drug treatments on $P$. aeruginosa growth were determined following co-culture experiments by diluting and plating coculture supernatants on Luria broth agar plates, using a micro-dilution plating technique (237). P. aeruginosa adherence to HT-29 cells was determined following a $3 \mathrm{~h}$ co-culture period by 
scraping cells into DPBS containing $0.6 \%$ BSA (DPBS-BSA), washing three times with DPBSBSA, lysing cells on ice in $0.25 \%$ Triton X-100, then diluting and plating the lysate. Number of bacteria per eukaryotic cell was calculated. The efficiency of T3S effector production and secretion in the presence of drugs was determined by harvesting co-culture supernatants and detecting ExoS-HA and PopB by SDS-PAGE and immunoblot analysis.

Immunofluorescence (IF) analyses - HT-29 cells were seeded at $1.5 \times 10^{5} \mathrm{cells} \mathrm{ml}^{-1}$ in chamber slides (Nalge Nunc International) $48 \mathrm{~h}$ prior to drug treatment and co-cultured with $\mathrm{Pa}$ ExoS-HA or enzymatically inactive ExoS for $3 \mathrm{~h}$. This shorter co-culture period allowed for bacterial-host cell interaction with limited bacterial effects on eukaryotic cell morphology. Cells were then fixed with $3 \%$ paraformaldehyde in actin stabilization buffer (ASB; (115), permeabilized with $0.2 \%$ Triton $\mathrm{X}-100$ in $\mathrm{ASB}$, blocked, and stained using monoclonal antibodies to: IQ-motif-containing GTPase-activating protein 1 (IQGAP1) (Invitrogen), a-tubulin (Invitrogen), Rac1 (BD Transduction Laboratories) or phosphatidylinositol 3-kinase (PI3K) (BD Transduction Laboratories) coupled with anti-mouse Alexa Fluor 488 conjugate (Invitrogen). Actin was stained using phalloidin conjugated to tetramethylrhodamine B isothiocyanate (Phalloidin-TRITC, Sigma-Aldrich). Lipid rafts were stained with Alexa Fluor 488-labeled cholera toxin B subunit (CTB, Invitrogen). P. aeruginosa was stained with a polyclonal guinea pig antibody against $P$. aeruginosa (Biogenesis) or a polyclonal rabbit antibody against $P$. aeruginosa LPS (provided by Joseph Lam, University of Guelph, Canada), followed by an appropriate Alexa Fluor conjugate (Invitrogen). Cells were mounted using Fluoromount-G (Southern Biotechnology Associates) and visualized using a Plan-Neofluar x100/1.3 oil objective on a Zeiss LSM 510 confocal microscope (Jena, Germany), or a Plan-Apochromat x63/1.40 oil objective on a Zeiss Imager.Z1 LSM 510 confocal microscope. Images were captured and exported as 8-bit TIF files into Adobe Photoshop CS2 (Adobe Systems Incorporated, San Jose, CA) and cropped. 
For IF staining of TPA-differentiated HL-60 (dHL-60) cells, cells were seeded on glass cover slips, differentiated with $20 \mathrm{nM}$ TPA for the indicated time, and then co-cultured for $3 \mathrm{~h}$ with PaExoS-HA. Samples were prepared for IF analysis and stained for the indicated proteins, as described above. For IF staining of undifferentiated, non-adherent T3S resistant HL-60 (rHL-60) cells or T3S sensitive (sHL-60) cells, cells were seeded in tubes, collected by centrifugation and prepared for IF analysis as above, with the exception that cells were centrifuged between washes and fixed to glass slides using a cytospin.

IQGAP1 siRNA knockdown - ON-TARGETplus SMARTpool RNA oligomers against human IQGAP1 (Dharmacon) were transfected into HT-29 cells using TransIT-TKO Transfection Reagent (Mirus Bio Corporation), according to the manufacturer's specifications. siRNA sequences targeting multiple IQGAP1 mRNA sequences included: GAACGUGGCUUAUGAGUAC, GCAGGUGGAUUACUAUAAA,

CGAACCAUCUUACUGAAUA, and (4) CAAUUGAGCAGUUCAGUUA. siCONTROL NonTargeting siRNA \#1 (Dharmacon) was used as a control. Cells were co-cultured with Pa-ExoSHA 48 or 72 h post-transfection. IQGAP1 expression was examined by Western blot analysis and quantified by densitometry using ImageJ, and percentage IQGAP1 expression was determined relative to quantified levels of IQGAP1 in non-drug treated cells that were cocultured with Pa-ExoS-HA.

Focal complex, focal adhesion, and LE functional analyses - To assess the role of focal complexes and focal adhesions in Pa-T3S sensitivity of adherent HT-29 cells, cells were seeded 24-48 $\mathrm{h}$ prior to treatment with the following inhibitors: (1) Rac1 inhibitor, NSC23766 (EMD Chemicals), added to cells $16 \mathrm{~h}$ prior to co-culture; (2) Rho-kinase (ROCK) inhibitor, Y-27632 (Alexis Biochemicals), added to cells $1 \mathrm{~h}$ prior to co-culture; (3) Src kinase inhibitor, PP2 (Calbiochem), added to cells $1 \mathrm{~h}$ prior to co-culture; or (4) Cdc42 inhibitor, secramine A $\left(\mathrm{C}_{35} \mathrm{H}_{39} \mathrm{BrN}_{2} \mathrm{O}_{5} \mathrm{~S}\right.$; provided by Thomas Kirchhausen, Harvard Medical School, MA, USA and 
Gerald B. Hammond, University of Louisville, KY, USA), added to cells in medium containing $0.5 \% \mathrm{BSA}$ and $1 \% \mathrm{DMSO} 1 \mathrm{~h}$ prior to co-culture. In examining the effects of inhibitors on nonadherent HT-29 cells, cells were seeded at $5.0 \times 10^{5}$ cells $\mathrm{ml}^{-1}$, followed by the immediate addition of drugs as described above. To assess the role of LE associated actin, microtubules (MTs) and PI3K in Pa-TS3, HT-29 cells were seeded as described above for pre- and postadherence drug treatment studies and treated with the following inhibitors for $1 \mathrm{~h}$ : latrunculin $\mathrm{B}$ (LatB; BIOMOL International), nocodazole (Sigma-Aldrich), AS605240, or LY294002 (Cayman Chemicals). To disrupt existing MTs, cells were placed at $4^{\circ} \mathrm{C}$ for $3 \mathrm{~h}$ prior to drug treatment. Cells were co-cultured with $\mathrm{Pa}-\mathrm{ExoS}-\mathrm{HA}$ in the presence of drug, and drug toxicity and $\mathrm{Pa}-\mathrm{T} 3 \mathrm{~S}$ sensitivity were examined as previously described.

Statistical analysis - Statistical significance was calculated based on a Student's $t$ test, using JMP, Version 7 Software (SAS Institute). The relationship between eukaryotic cell adherence to tissue culture wells and Pa-T3S effector translocation was computed using a Pearson's correlation coefficient based on comparisons of percentage adherence with percentage RalA modification for individual samples under the indicated experimental conditions. 


\section{RESULTS}

Inhibition of host cell Pa-T3S sensitivity. The finding that most cultured cells are sensitive to Pa-T3S underscores the conserved nature of host cell properties conferring T3S sensitivity. To help characterize these properties, cell lines were treated with agents that alter membrane properties, cytoskeletal structure, or cell signaling pathways, and their effects on host cell $\mathrm{Pa}$ T3S sensitivity were determined. Pa-T3S sensitivity was monitored based on effector internalization following co-culture with $P$. aeruginosa strains expressing the T3S effector, ExoS. Of the many agents tested (summarized Supplementary Table, S1), two membrane modulating agents, $M \beta C D$ and PFO were identified to inhibit host cell Pa-T3S-sensitivity. Similar results were obtained with HT-29, T24, and other tested Pa-T3S sensitive cell lines. The finding that agents modulating membrane structure, but not those altering cytoskeletal structure or common cell signaling pathways, affected T3S sensitivity provided an initial indication that host cell sensitivity to Pa-T3S was linked to membrane properties.

With the identification of $\mathrm{M} \beta \mathrm{CD}$ and PFO as Pa-T3S inhibitory agents, these agents were then used as tools to characterize alterations in host cell properties associated with loss of Pa-T3S sensitivity. $M \beta C D$ depletes membrane cholesterol, and in dose-response studies, treatment of HT-29 epithelial cells with 2-3 mM M $\beta C D$, prior to co-culture with Pa-ExoS-HA, caused a decrease in T3S effector ExoS translocation (Fig. 8a, indicated by asterisks). The decrease in ExoS translocation corresponded with a decreased shift in the apparent molecular mass of RalA, reflecting the decreased internalization and functional ADP ribosylation of host cell RalA by ExoS. As shown in Fig. 8(a), RalA exhibits variable shifts in molecular mass upon ADP ribosylation by ExoS. This relates to RalA having primary (Arg52) and secondary (Arg135 and Arg161) sites of ExoS ADP ribosylation, which results in one or two shifts in RalA as assayed by SDS-PAGE and Western blot analysis $(96,270)$. Quantification of the shift in molecular mass of RalA (determined by densitometry) can in turn be used as a read-out of the efficiency of ExoS 
effector translocation $(96,270)$. M $3 C D$ also caused a dose-dependent decrease in the translocation of enzymatically inactive ExoS (a GAP/ADPRT mutant), but inhibition required a slightly higher, 4-5 mM, concentration of $M \beta C D$ (data not shown). This finding suggests that the mechanism underlying the inhibitory effects of $\mathrm{M} \beta C D$ on Pa-T3S is somehow augmented by ExoS.

Inhibition of $\mathrm{Pa}$-T3S by $\mathrm{M} \beta \mathrm{CD}$ occurred in the absence of major effects on HT-29 cell viability as determined by trypan blue exclusion assays. However, a decrease in HT-29 cell adherence to tissue culture wells (a $29-34 \%$ loss of adherence) occurred in conjunction with a decrease in T3S sensitivity (initial decrease in adherence indicated by an asterisk in Fig. 8a). Membrane fractionation studies confirmed that inhibition of $P a-T 3 S$ by $M \beta C D$ occurred at the level of $P a$ T3S translocon insertion and effector translocation, as apparent in the decrease in PopB and ExoS in membrane fractions of $M \beta C D$ treated cells (Fig. 8b). Effects of $M \beta C D$ were also targeted to host cells, as evident by the lack of effect of M $\beta C D$ on T3S effector/translocator production (Fig. 8c), or on P. aeruginosa growth or adherence to host cells (Fig. 8d), at $3 \mathrm{mM}$ inhibitory concentrations. Inhibitory effects of $\mathrm{M} \beta C D$ on Pa-T3S were consistent with the findings of other Gram-negative bacteria that support the role of cholesterol-rich membrane rafts in T3S $(132,259,318)$. CTB binds GM1 gangliosides that partition to lipid rafts, and when coupled to Alexa Fluor 488 can be used to monitor alterations in lipid rafts relative to increasing concentrations of $M \beta C D$ (337). Previous studies using CTB to track lipid rafts found increasing concentrations of $\mathrm{M} \beta C D$ to disrupt lipid raft organization (180). When CTB was used to monitor lipid rafts in HT-29 cells relative to increasing concentrations of $M \beta C D$ and inhibition of T3S, CTB staining changed from a diffuse punctate pattern to an aggregated pattern at $0.5 \mathrm{mM}$ $M \beta C D$, a concentration of $M \beta C D$ lower than that required to inhibit T3S. These results indicated that lipid raft reorganization preceded inhibition of $\mathrm{Pa}$-T3S. Our conclusions from studies using 
$M \beta C D$ to identify host cell properties involved in T3S sensitivity were that: (1) membrane cholesterol influences Pa-T3S translocon insertion, and (2) membrane alterations more severe than lipid raft reorganization were required to inhibit T3S. 
Figure 8. Effect of M $\beta C D$ treatment on Pa-T3S sensitivity. HT-29 cells were treated with the indicated concentration of $\mathrm{M} \beta C D$ for $30 \mathrm{~min}$, prior to and during co-culture with strain $\mathrm{Pa}$-ExoSHA. (a) To assay for Pa-T3S sensitivity, HT-29 cell extracts were immunoblotted for translocation of T3S effector, ExoS and for internalized effector function based on ExoS ADP ribosylation of RalA (as recognized by a shift in molecular mass of RalA). GAPDH served as a protein expression/loading control. Percent viable and adherent cells were enumerated using trypan blue staining. Asterisks mark initial decreases in Pa-T3S sensitivity and loss of HT-29 cell adherence. (b) Effects of $\mathrm{M} \beta C D$ on Pa-T3S translocon insertion were assessed by immunoblotting HT-29 cell membrane fractions for PopB and ExoS. Inhibition of Pa-T3S coincided with interference of PopB translocon and ExoS membrane insertion. PA103 $\triangle \mathrm{PopB}$ served as a translocon mutant control in these studies. sn, T3S induced $P$. aeruginosa culture supernatant used as a molecular mass marker for ExoS and PopB. (c) Effects of M $\mathrm{BCD}$ on $P$. aeruginosa induction and production of T3S effectors were determined by harvesting and assaying co-culture supernatants for PopB and ExoS by immunoblot analysis. (d) Effects of M $\beta C D$ on $P$. aeruginosa growth were determined by quantifying $P$. aeruginosa c.f.u. in coculture supernatants at the end of the culture period. Results are expressed as the ratio (mean $\pm S D$ ) of c.f.u. of MBCD-treated vs. non-drug treated cells, co-cultured with Pa-ExoS-HA. Effects of $\mathrm{M} \beta \mathrm{CD}$ on $P$. aeruginosa-adherence to HT-29 cells were determined following the co-culture period by harvesting and plating HT-29 cell lysates to quantify c.f.u. Results are expressed as number (mean \pm SEM $\times 10^{-3}$ ) of $P$. aeruginosa adhering per HT-29 cell. (e) The relationship between the effects of $M \beta C D$ on Pa-T3S sensitivity and host cell lipid raft structure was determined following co-culture with $\mathrm{Pa}$-ExoS-HA and using Alexa Fluor 488-labeled CTB to stain GM1 gangliosides that partition to lipid rafts. Results of all studies are representative of three to six independent experiments. Bars, $10 \mu \mathrm{m}$. 


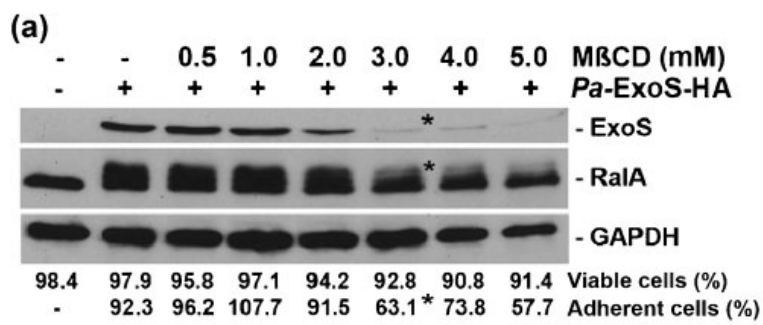

(b)

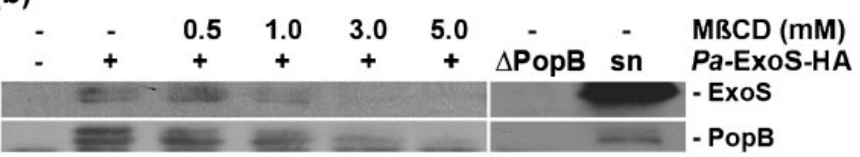

(c)

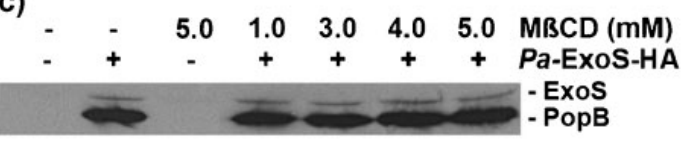

(d)

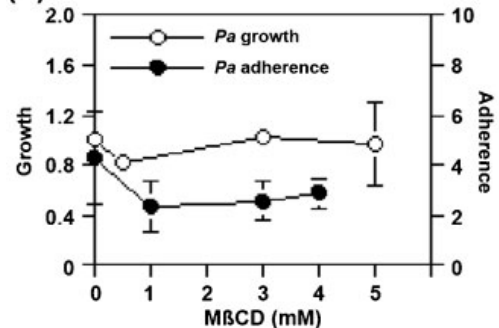

(e)

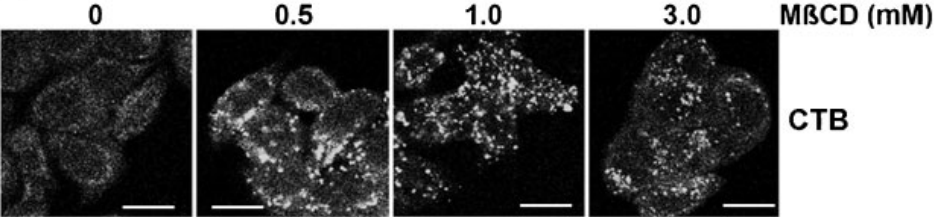


PFO affects membrane properties differently than $M \beta C D$ causing oligomerization of membrane cholesterol in conjunction with pore formation (310). PFO was found to inhibit HT-29 cell sensitivity to Pa-T3S in a dose-dependent range of 0.04-0.2 $\mu \mathrm{g} \mathrm{PFO} \mathrm{ml}^{-1}$ (Fig. 9a) and was used to further examine host cell properties linked to Pa-T3S sensitivity. As observed with $M \beta C D$, inhibition of Pa-T3S by PFO occurred in the absence of major effects on HT-29 cell viability but in conjunction with loss of HT-29 cell adherence to tissue culture wells (a $26-52 \%$ loss of adherence), which was greater than that observed for M $\beta C D$ (Fig. 9a). PFO also caused a dose-dependent decrease in the translocation of enzymatically inactive ExoS, but inhibition required a slightly higher, 0.3-0.4 $\mu \mathrm{g} \mathrm{ml}^{-1}$, concentration of PFO (results not shown). The increased toxic effects of PFO on HT-29 cell adherence limited the co-culture time, which was reflected in decreased detection of ExoS in cell extracts. In membrane fractionation studies, increasing concentrations of PFO caused translocator protein PopB and effector ExoS to accumulate in membranes rather than to decrease, as was observed with $M \beta C D$ (Fig. 9b). PFO was also confirmed to target host cell properties based on its lack of effect on T3S effector/translocator production (Fig. 9c) or on $P$. aeruginosa growth or adherence to host cells (Fig. 9d). Comparisons of effects of M $\beta C D$ and PFO on T3S thus found depletion of membrane cholesterol by $M \beta C D$ to interfere with $T 3 S$ translocon insertion, while oligomerization of membrane cholesterol by PFO allowed translocon insertion but not effector translocation.

Cholesterol-dependent cytolysins, such as PFO, have been reported to bind and serve as probes for cholesterol-rich lipid rafts $(223,293,330)$. When CTB was used to track how PFO altered lipid rafts in relation to inhibition of Pa-T3S, lipid raft reorganization occurred at sub-T3S inhibitory concentrations $\left(0.02 \mu \mathrm{g} \mathrm{ml}^{-1}\right)$ of PFO (Fig. 9e). More severe aggregation and eventual loss of lipid raft staining occurred at T3S inhibitory concentrations (0.1-0.2 $\left.\mu \mathrm{g} \mathrm{m}^{-1}\right)$ of PFO. These findings are consistent with studies of the cholesterol-dependent cytolysin, listeriolysin $\mathrm{O}$, 
which was found to be a potent aggregator of lipid rafts (112). The fact that alterations in lipid rafts by both PFO and $M \beta C D$ preceded inhibition of T3S sensitivity provided evidence that lipid raft organization was indirectly related to host cell Pa-T3S sensitivity.

PFO pore formation has been dissected into distinct steps: (1) binding of PFO monomers to the eukaryotic cell receptor, cholesterol; (2) oligomerization of PFO monomers in association with membrane cholesterol to form a pre-pore complex; and (3) pore formation, associated with the extension of amphipathic transmembrane $\beta$-hairpins (310). We were able to clarify the stage in PFO function inhibiting Pa-T3S through the use of PFO mutants locked at either the cholesterol monomer-binding step (SSPFO) (250) or at the cholesterol oligomerization pre-pore complex step (dsPFO) (144). Treatment of HT-29 cells with ssPFO or dsPFO, prior to exposure to PaExoS-HA, caused no inhibition of T3S, even at concentrations 1000-fold higher than that of wild type PFO (Fig. 9f). Collectively, our conclusions from using PFO as a T3S inhibitory tool were that: (1) PFO interrupted T3S effector translocon function but not membrane insertion, differing from the effects of $M \beta C D$; and (2) neither binding to nor oligomerization of membrane cholesterol, or the redistribution of lipid rafts by PFO appeared to be sufficient to interfere with Pa-T3S. Rather, alterations in membrane properties associated with intact PFO were required to inhibit Pa-T3S in HT-29 cells.

To summarize, studies of T3S sensitive cells provided evidence that cellular properties linked to membrane structure were involved in host cell Pa-T3S sensitivity, and that membrane cholesterol influenced T3S translocon insertion and function. What remained unclear was the mechanism underlying the role of membrane structure in Pa-T3S sensitivity, although M $\beta C D$ or PFO treatment found cholesterol or lipid raft reorganization to be insufficient to interrupt host cell Pa-T3S sensitivity. 
Figure 9. Effect of PFO treatment on Pa-T3S sensitivity. HT-29 cells were treated with the indicated concentration of PFO $1 \mathrm{~h}$ prior to and during co-culture with strain Pa-ExoS-HA. Effects of PFO on: (a) inhibition of Pa-T3S, (b) Pa-T3S translocon insertion, (c) P. aeruginosa induction and production of T3S effectors, (d) $P$. aeruginosa growth and $P$. aeruginosaadherence to HT-29 cells, and (e) lipid raft structure were determined as described in Fig. 8. In membrane fractionation studies, an increase in PopB and ExoS membrane association occurred relative to increasing concentrations of PFO, indicating that PFO inhibited ExoS translocation and translocon function after membrane insertion. (f) To examine the stage in PFO function inhibiting Pa-T3S, HT-29 cells were treated with wild type PFO, ssPFO or dsPFO for 1 h prior to and during co-culture with strain Pa-ExoS-HA. Pa-T3S was assayed based on ExoS ADP ribosylation of RalA, and asterisks indicate initial decreases in RalA ADP ribosylation and loss of HT-29 cell adherence to tissue culture wells. Results of all studies are representative of three to six independent experiments. Bars, $10 \mu \mathrm{m}$. 

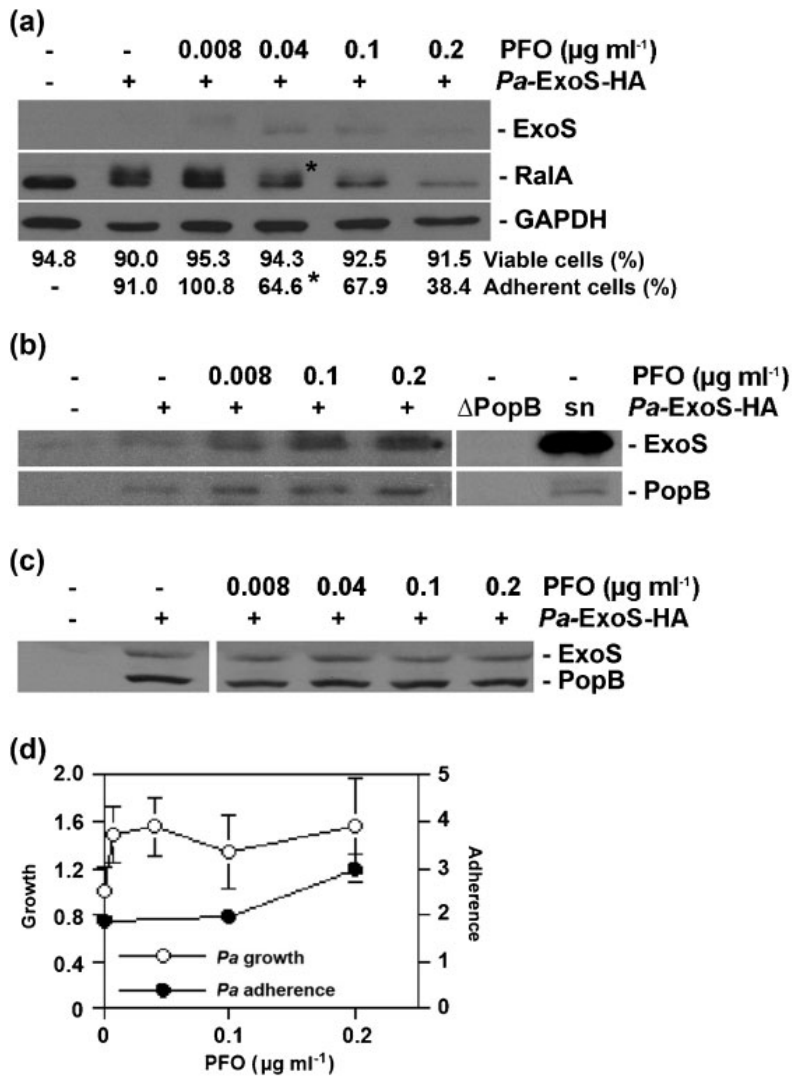

(e)

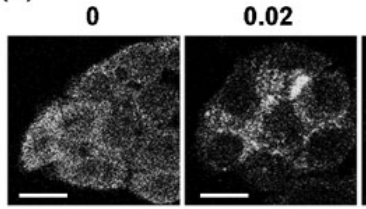

0.1 0.2 PFO $\left(\mu \mathrm{g} \mathrm{ml}^{-1}\right)$

(f)

\begin{tabular}{|c|c|c|c|c|c|c|c|c|c|}
\hline \multicolumn{3}{|c|}{ PFO } & \multicolumn{3}{|c|}{ ssPFO } & \multicolumn{3}{|c|}{ dsPFO } & \\
\hline $\begin{array}{c}0.008 \\
+\end{array}$ & $\begin{array}{c}0.04 \\
+\end{array}$ & $\begin{array}{c}0.4 \\
+\end{array}$ & $\begin{array}{c}0.4 \\
+\end{array}$ & $\begin{array}{c}4.0 \\
+\end{array}$ & $\begin{array}{r}40 \\
+\end{array}$ & $\begin{array}{c}0.4 \\
+\end{array}$ & $\begin{array}{c}4.0 \\
+\end{array}$ & $\begin{array}{l}40 \\
+\end{array}$ & $\begin{array}{l}\left(\mu \mathrm{g} \mathrm{ml}^{-1}\right) \\
\text { Pa-ExoS-HA }\end{array}$ \\
\hline 8 & $=$ & 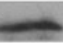 & de & $m$ & $=$ & 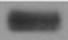 & en & $\mathrm{mos}$ & - RalA \\
\hline
\end{tabular}


Induction of Pa-T3S sensitivity in HL-60 cells. Promyelocytic HL-60 cells are innately resistant to Pa-T3S (rHL-60 cells) but can be induced to become T3S-sensitive by treatment with the differentiation agent, TPA (dHL-60 cells) (271). It was also observed that HL-60 cells acquire sensitivity to Pa-T3S in culture after multiple passages (sHL-60 cells). We therefore used HL-60 cells as an alternative model system to study host cell properties involved in $\mathrm{Pa}$ T3S by monitoring cellular alterations in $\mathrm{rHL}-60, \mathrm{sHL}-60$, and $\mathrm{dHL}-60$ cells. Although the stimulus that induces sHL-60 cell Pa-T3S sensitivity is unknown, TPA differentiation of HL-60 cells has been well studied and causes HL-60 cells to differentiate from round, non-adherent promyelocytes to adherent macrophages that exhibit properties of polarized migrating cells (47, 184). TPA differentiation of HL-60 cells is known to involve activation of protein kinase $\mathrm{C}$ (PKC $\beta$ ) signaling pathways, which induce early (after $15 \mathrm{~min})$, intermediate $(2-3 \mathrm{~h})$ and late (12-24 h) patterns of gene expression $(295,307,355)$. Relating temporal patterns of TPA-induced gene expression to induction of T3S sensitivity in turn provided another means of gaining insight into host cell properties involved in Pa-T3S sensitivity.

When HL-60 cells were exposed to $20 \mathrm{nM}$ TPA and then co-cultured with Pa-ExoS-HA, induction of T3S sensitivity occurred after a 12-24 h exposure to TPA, as assayed based on ExoS translocation and ADP ribosylation of RalA (Fig. 10a). In examining the relationship between induction of T3S sensitivity and T3S translocon function, ExoS membrane translocation was found to coincide first with $\mathrm{PopB}$ and then with PopD translocon protein membrane insertion (Fig. 10a). This finding indicated that HL-60 cell resistance to Pa-T3S related to membrane properties that affected T3S translocon insertion, which similar to HT-29 cells, identified a role for membrane properties in T3S translocon insertion and function. The requirement for a 12-24 $\mathrm{h}$ exposure to TPA for induction of T3S sensitivity indicated that expression of late TPA response genes, which includes actin binding and adherence-related proteins, were involved in cellular alterations affecting T3S sensitivity. 
To further understand host cell properties involved in Pa-T3S sensitivity, we monitored alterations in HL-60 cell architecture relative to induction of T3S sensitivity by staining for the cytoskeletal proteins actin and tubulin. Pa-T3S-resistant rHL-60 cells were characterized by dense cortical actin staining and weak tubulin staining that lacked pronounced MT radiations (Fig. 10b). sHL-60 cells maintained a round, non-adherent morphology, indistinguishable from that of rHL-60 cells by phase-contrast microscopy. However, upon staining for actin and tubulin, sHL-60 cells exhibited pronounced MT radiations, which identifies an early stage in the induction of directional migration in HL-60 cells. MT radiations were also evident in a small population of HL-60 cells exposed to TPA for $12 \mathrm{~h}$, but the frequency of this phenotype was not sufficient to detect effector translocation by immunoblot analysis. After 18 and $24 \mathrm{~h}$ exposure to TPA, dHL-60 cells exhibited extensive tubulin radiations and actin-enriched projections that defined a mature migratory phenotype. At this stage in TPA differentiation, dHL-60 cells were highly sensitive to Pa-T3S as documented by immunoblot analyses at $24 \mathrm{~h}$ (Fig. 10a). To summarize, in studies of HL-60 cell architecture it became evident that MT radiations and actinrich projections, characteristic of polarized migrating cells, occurred in conjunction with induction of T3S sensitivity. 
Figure 10. Examining the induction of Pa-T3S sensitivity in HL-60 cells. (a) Pa-T3Sresistant rHL-60 cells were treated with $20 \mathrm{nM}$ TPA for the indicated time, prior to co-culture with Pa-ExoS-HA. Cells were lysed and fractionated, and the membrane fraction was resolved by SDS-PAGE and immunoblotted for ExoS and RalA to assay T3S effector translocation and function, and for PopB and PopD to assess T3S translocon membrane insertion. sn, $P$. aeruginosa culture supernatant used as molecular mass markers for ExoS, PopB, and PopD. (b) The morphology of rHL-60 cells, T3S-sensitive sHL-60 cells, and TPA differentiated dHL-60 cells (differentiated for 12, 18, or $24 \mathrm{~h}$ ) was examined by IF staining for tubulin (green) and actin (red). Microtubule radiations developed during early stages of Pa-T3S sensitivity and continued to develop in $\mathrm{dHL}-60$ cells in association with actin enriched projections and induction of Pa-T3S sensitivity. Images are representative of common phenotypes observed under the indicated experimental conditions in two or more experiments. Bar, $10 \mu \mathrm{m}$. 

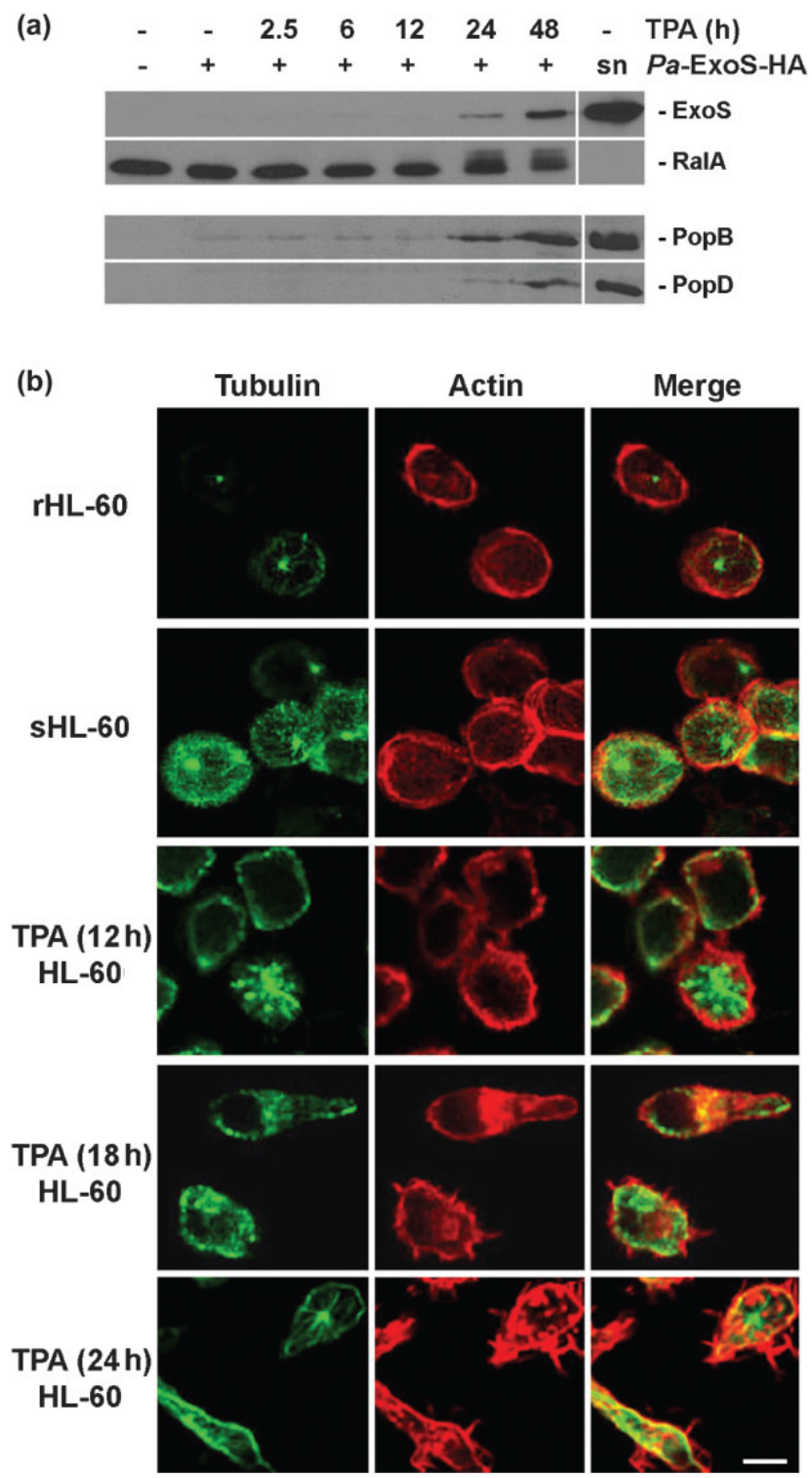
Association of eukaryotic cell adhesion processes with Pa-T3S sensitivity. The complex nature of bacterial-host cell interactions during T3S has made it experimentally difficult to determine how eukaryotic cell properties contribute to T3S translocon function. The serendipitous finding in studies of $P$. aeruginosa that helped bridge this understanding was that eukaryotic cells acquire or lose adherence to tissue culture wells in conjunction with the gain or loss Pa-T3S sensitivity. TPA induces HL-60 cells to differentiate from non-adherent promyelocytes to adherent macrophages $(47,184)$. In relating HL-60 cell adherence to Pa-T3S sensitivity, approximately $70 \%$ of HL-60 cells became adherent to tissue culture wells following a $24 \mathrm{~h}$ exposure to TPA. To further examine this relationship, TPA induction of HL-60 cell T3S sensitivity was directly compared with induction of HL-60 cell adherence in samples run in parallel, using ExoS translocation and ADP ribosylation RalA to quantify the efficiency of T3S. In these studies, a positive correlation ( $r=0.8146$; determined using Pearson's correlation coefficient) was detected between the percentage of adherent HL-60 cells and the efficiency of T3S effector translocation (shown in Supplementary Fig. S1a). Studies of TPA differentiated HL60 cells thus provided an initial indication that the relationship between eukaryotic cell adherence mechanisms and Pa-T3S was statistically relevant.

We also observed that HT-29 epithelial cells lost adherence to tissue culture wells in association with inhibitory effects of M $\beta C D$ and PFO on Pa-T3S (Figs. 8a and 9a). To determine whether a reverse correlation between inhibition of $\mathrm{Pa}$-T3S directly and loss of adherence was evident, we used a similar approach to examine adherence and T3S sensitivity in HT-29 cells. In these studies, a positive correlation was observed between the loss of HT-29 cell adherence to tissue culture wells and the inhibitory effects of M $\beta C D(r=0.8651)$ and PFO $(r=0.7836)$ on Pa-T3S sensitivity (Supplementary Figs. S1b, c). The finding that inhibition of HT-29 cell Pa-T3S sensitivity correlated with loss of adherence to tissue culture wells, and conversely that induction of HL-60 cell T3S sensitivity correlated with acquisition of adherence, provided 
evidence that eukaryotic cell adherence mechanisms and Pa-T3S sensitivity might somehow be functionally linked. The importance of this finding to our studies was that it directed our attention to the use of eukaryotic cell adherence mechanisms in further analyses of host cell involvement in Pa-T3S.

\section{Examining the type of adhesion mechanism involved in host cell Pa-T3S sensitivity.}

Wounded epithelial barriers, which are sensitive to $P$. aeruginosa infections and Pa-T3S, undergo cell migration in response to the wound healing process. Fig. 11(a) diagrams the organizational structure of LE proteins that drive cell movement. Rac1 mediates adherence at the LE through the formation of dynamic, focal complexes (222). Focal complexes can mature into more stable Rho-mediated focal adhesions (as shown in Supplementary Fig. S2a), that form behind focal complexes and at the rear of cells, where they serve as traction points in cell migration (256). Based on evidence that host cell adherence to tissue culture wells correlated with Pa-T3S sensitivity, we next used inhibitors that target proteins within focal complexes or focal adhesions to examine whether these adhesion mechanisms were involved in Pa-T3S. Inhibitors that were used to test the involvement of focal complexes in Pa-T3S included: (1) NSC23766, which inhibits Rac1 function by blocking Rac1 interaction with its GEF (107); or (2) secramine $A$, which interferes with Cdc42 binding to membranes in a RhoGDI-dependent manner $(234,340)$. When HT-29 cells were treated with Rac1 or Cdc42 inhibitors, prior to coculture with Pa-ExoS-HA, dose-dependent decreases in effector translocation were observed (Fig. 11a, black bars), with significant decreases in T3S $(P<0.05-0.025)$ occurring at higher drug concentrations. Treatment with both Rac1 and Cdc42 inhibitors together did not augment inhibitory effects on Pa-T3S (results not shown). Inhibitors used to test the role of focal adhesions in Pa-T3S included: (1) Y-27632, an ATP-competitive inhibitor of p160ROCK (61); or (2) PP2, a Src kinase inhibitor. Unlike treatments that affect focal complexes, ROCK inhibitor at 4 times its inhibitory concentration, and Src kinase inhibitor at concentrations as high as $80 \mu \mathrm{m}$, 
caused no dose-dependent or significant inhibition of Pa-T3S (Supplementary Fig. S2b, black bars). None of the inhibitors affected $P$. aeruginosa growth or secretion of T3S proteins.

The correlation between cell adherence and $\mathrm{Pa}$-T3S sensitivity led us to examine whether eukaryotic cell adherence processes stabilize T3S translocon function. To test this possibility, HT-29 cells were treated with Rac1, Cdc42, ROCK, or Src kinase inhibitors, as described above, but inhibitors were added at the time of HT-29 cell seeding, prior to establishment of adherence. When treated with inhibitors before adherence, HT-29 cells remained non- or loosely adherent during co-culture with Pa-ExoS-HA. As shown in Fig. 11(a) (gray bars), both Rac1 and Cdc42 inhibitors caused a greater inhibition of T3S effector translocation when applied to non-adherent as compared to adherent HT-29 cells. In contrast, no significant or dose-dependent inhibition of T3S effector translocation was detected when ROCK or Src kinase inhibitors were applied prior to establishment of adherence (Supplementary Fig. S2b, gray bars). Additional evidence supporting the lack of involvement of focal adhesions in Pa-T3S included: (1) the inability of antibodies that block integrin signaling to interfere with Pa-T3S (results not shown), and (2) the finding that SYF fibroblastic cells that are deficient for all three Src kinases, Src, Fyn, and Yes (162) were sensitive to Pa-T3S (results not shown). Together these studies favored a role of LE focal complexes, over that of focal adhesions, in host cell PaT3S sensitivity, and also provided evidence that host cell adherence processes stabilize Pa-T3S secretion.

Examining the relationship between host cell LE properties and Pa-T3S. If LE focal complexes contribute to host cell Pa-T3S sensitivity, other LE associated proteins, as shown in Fig. 11(a), might be predicted to influence T3S effector translocation. To allow for a stabilizing effect of cell adherence on Pa-T3S, HT-29 cells were treated at the time of seeding, prior to establishment of adherence, or $48 \mathrm{~h}$ after adherence with: (1) LatB to inhibit actin polymerization; (2) nocodazole, combined with incubation at $4^{\circ} \mathrm{C}$, to destabilize and inhibit MT 
formation; (3) LY294002 to inhibit all PI3K isoforms; or (4) AS605240 to inhibit the $\gamma$-isoform of PI3K involved in cell motility $(19,41)$. Following drug treatment cells were co-cultured with $\mathrm{Pa}$ ExoS-HA, and host cell T3S sensitivity was quantified based on the efficiency of ExoS translocation and ADP ribosylation RalA. As shown in Fig. 11(b) (black bars), and consistent with the data in Supplementary Table S1, most of these treatments caused no significant decrease in T3S effector translocation when applied to adherent HT-29 cells. The one exception was $100 \mu \mathrm{M}$ LatB, which caused a significant $(P<0.02)$ decrease in T3S effector translocation. When applied to non-adherent HT-29 cells, both 10 and $100 \mu \mathrm{M}$ LatB caused a significant decrease $(P<0.002)$ in T3S effector translocation (Fig. 11b, gray bars), as did $10 \mu \mathrm{M}$ nocodazole $(P<0.02)$, implicating a role of MTs in Pa-T3S. The role of PI3K in Pa-T3S was less evident with $200 \mu \mathrm{M}$ LY294002 causing a decrease in effector translocation, but this did not attain statistical significance. The finding that the roles of actin, MTs, and PI3K in T3S effector translocation paralleled their organizational structure at the LE provided further validation of the role of LE properties in Pa-T3S. Also, the increased effectiveness of inhibitors when applied to nonadherent HT-29 cells was again supportive of LE adherence exerting a stabilizing role in $\mathrm{Pa}$ T3S translocation.

IQGAP1 localizes to the LE, where it functions as a scaffolding protein during cell migration, binding directly to F-actin, Cdc42, and Rac1, and indirectly capturing MT plus ends during cell migration $(21,104,127)$. The role of LE IQGAP1 in cell motility was confirmed in IQGAP1 knockdown (siRNA), which led to suppressed cell migration (199). To further examine whether LE properties associated with cell migration contributed to host cell Pa-T3S sensitivity, transient IQGAP1 siRNA knockdown studies were performed in HT-29 cells, and IQGAP1 expression was related to the efficiency of $\mathrm{Pa}$-T3S effector translocation following co-culture with $\mathrm{Pa}$-ExoSHA. When IQGAP1 expression was quantified by densitometry in siRNA dose-response studies, a significant decrease in IQGAP1 was detected at $5 \mathrm{nM}$ IQGAP1 siRNA $(P<0.001)$, with non- 
targeting siRNA studies requiring 20-fold higher concentrations to decrease IQGAP1 expression (see Supplementary Fig. S3). Significant decreases $(P<0.001)$ in T3S effector translocation relative to non-targeting siRNA occurred at $100 \mathrm{nM}$ IQGAP1 siRNA. The fact that transient siRNA expression studies were performed on adherent HT-29 cells, yet still a significant reduction in Pa-T3S was detected, is supportive of IQGAP1 playing a role in Pa-T3S.

Collectively, the model developing for host cell involvement in Pa-T3S was that membrane properties linked to eukaryotic cell adhesion mechanisms played a stabilizing role in T3S effector translocation. In studies examining the type of adhesion mechanism involved, a hierarchal order of protein involvement reflecting the architecture of the LE was observed with: (1) actin, Rac1 and Cdc42 playing a more direct role in Pa-T3S, (2) MTs and IQGAP1 playing secondary roles, and (3) PI3K playing a more distant role in Pa-T3S. 
Figure 11. Examining adherence mechanisms and LE properties involved in host cell PaT3S sensitivity. (a) Diagram of major eukaryotic cell proteins mediating LE focal complex adherence. Proteins enclosed in solid lined boxes were found to mediate Pa-T3S sensitivity based on inhibition studies. To examine the role of focal complexes in Pa-T3S, HT-29 cells were seeded and allowed to adhere for $48 \mathrm{~h}$ (black bars), before treatment with Rac1 inhibitor (NSC23766) or Cdc42 inhibitor (secramine A), or were treated with inhibitors in the same manner immediately following seeding prior to establishment of adherence (gray bars). Cells were then co-cultured with Pa-ExoS-HA in the presence of inhibitors, and Pa-T3S sensitivity was quantified in cell lysates based on the efficiency of ExoS ADP ribosylation of RalA. The mean \pm SEM of three independent experiments is graphed. Significant decreases relative to non-drug treated controls are indicated by ${ }^{*}(P<0.05)$ or ${ }^{* *}(P<0.025)$. (b) To examine the role of LE properties in Pa-T3S sensitivity, HT-29 cells were treated with the indicated inhibitors, either $48 \mathrm{~h}$ after (black bars) or immediately following seeding (gray bars) and co-cultured with PaExoS-HA and assayed for Pa-T3S sensitivity as in (a). Inhibitor treatments included: LatB, to disrupt actin; nocodazole, combined with incubation at $4^{\circ} \mathrm{C}$ for $3 \mathrm{~h}$ to destabilize/disrupt MTs; AS605240, to inhibit PI3K $;$; and LY294002, to inhibit all PI3K isoforms. Drug concentration relative to the efficiency of $\mathrm{Pa}$-T3S effector translocation is graphed as in (a), and the mean \pm SEM of five-seven independent experiments are represented. Significant decreases in RalA ADP ribosylation relative to non-drug treated controls are indicated by ${ }^{*}(P<0.02)$ or ${ }^{* *}(P<0.002)$. 
(a)
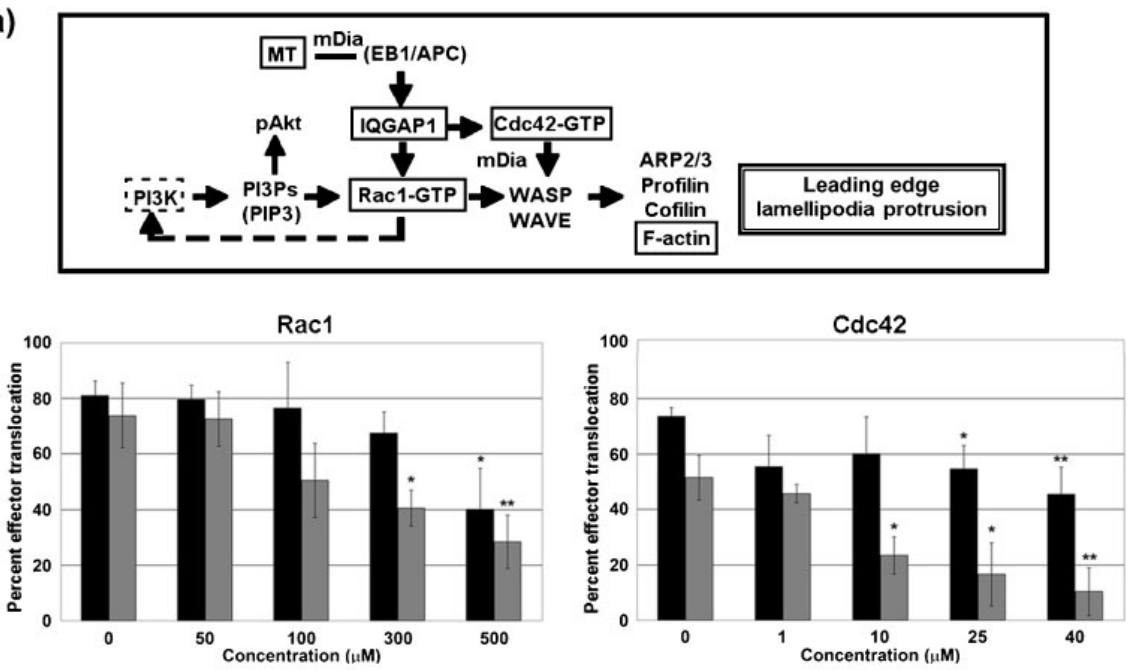

(b)

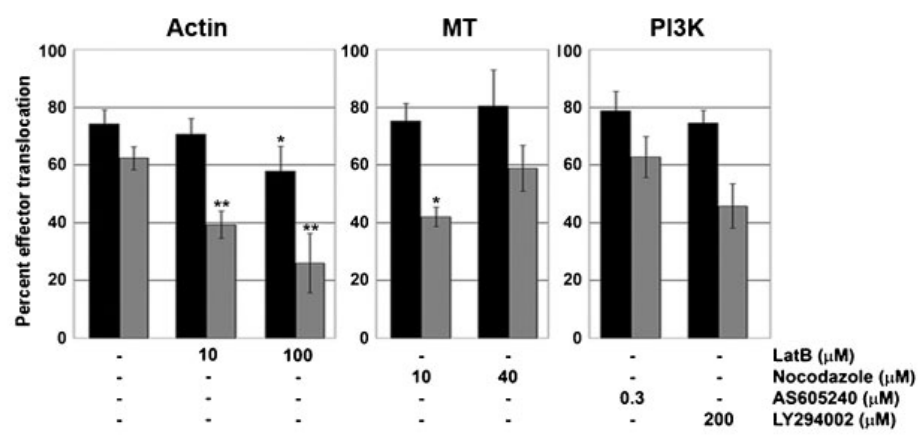

Cells seeded $48 \mathrm{~h}$ prior to treatment with drug and $\mathrm{Pa}$-ExoS-HA

Cells seeded $0 \mathrm{~h}$ prior to treatment with drug and $\mathrm{Pa}$-ExoS-HA 
Examining host cell architecture associated with Pa-T3S sensitivity. The LE is a functional assemblage of proteins that leads to cortical actin polymerization, which exerts a force on the plasma membrane to drive cell movement. Because of its dependence on assembly for function, the preferred method for examining LE function is through IF microscopy of LE architecture. The ability to alter Pa-T3S sensitivity in both HL-60 cells and HT-29 cells provided systems to monitor alterations in LE architecture relative to induction or inhibition of host cell T3S sensitivity.

HL-60 cell model - HL-60 cells allow LE architecture to be examined relative to stages in induction of Pa-T3S sensitivity, as defined by rHL-60, sHL-60, and dHL-60 cells. Host cell proteins that were visualized by IF staining for their cellular localization relative to alterations in Pa-T3S sensitivity included: (1) LE-associated Rac, IQGAP1, and PI3K, (2) focal adhesionassociated, Rho, and (3) actin. Supplementary Fig. S4(a) shows representative images from T3S-resistant rHL-60 cells, which consistent with their undifferentiated state, exhibited limited motility and LE properties, and lacked actin-enriched membrane structures, such as lamellipodia or filopodia. Rac distribution was cytosolic and did not co-localize with actin. IQGAP1 localized to the cell periphery, and as a scaffolding protein, co-localized with cortical actin (represented by yellow staining in the merged image). Localization of PI3K, which serves as a marker for polarization, resembled that of Rac1. Rho exhibited a polarized cytosolic staining pattern and did not co-localize with actin. In comparing staining patterns of rHL-60 with sHL-60 cells, the major differences observed in sHL-60 cells were that: (1) Rac1 staining became co-localized with actin, and (2) PI3K staining became more polarized (Supplementary Fig. S4a, b, indicated by arrows). Co-localization of Rac1 with actin and the polarized localization of PI3K serve as markers for the induction of functional LE properties. The finding that Rho localization was not altered in SHL-60 cells indicated that focal adhesion properties were not being modulated in conjunction with induction of $\mathrm{Pa}$-T3S sensitivity. 
Binding of $P$. aeruginosa to LE associated Rac, IQGAP1 and actin in T3S-sensitive dHL-60 cells is shown in Fig. 12. HL-60 cells treated with TPA for 18h developed mature LE structures, as evident in Rac and IQGAP1 staining patterns, which co-localize with actin in association with lamellipodia and cell motility projections. P. aeruginosa was found to bind to actin-rich LE projections at a $44.8 \%$ frequency and at sites of high-intensity Rac staining at a $78.5 \%$ frequency, when compared with $P$. aeruginosa association with dHL-60 cells in general. (Frequencies of $P$. aeruginosa association with LE regions were derived from 13 co-culture experiments and observation of 289 bacterial-eukaryotic cell associations.) Our conclusions from studies of LE architecture in HL-60 cells were that: (1) induction and development of Rac1, IQGAP1 and actin LE structures coincided with induction of T3S sensitivity, and (2) LE cell properties preferentially underlied $P$. aeruginosa binding in T3S cells. 

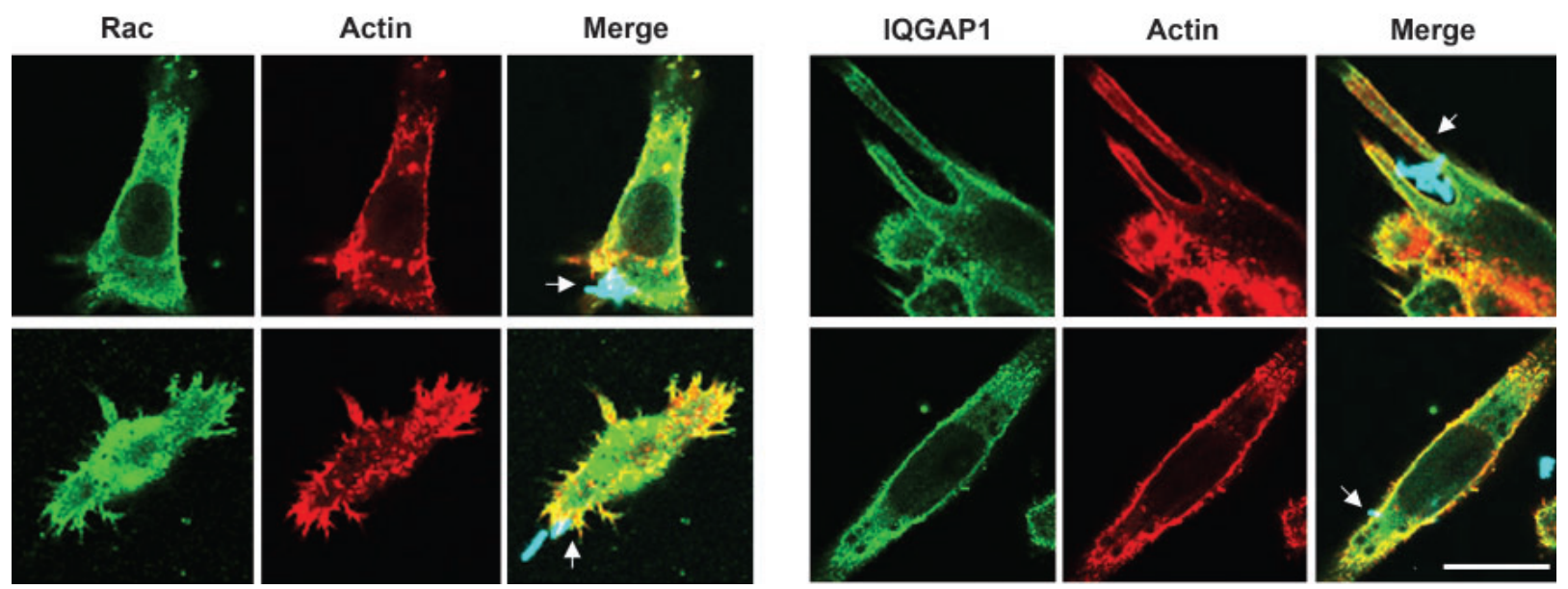

Figure 12. $P$. aeruginosa binding to LE of T3S-sensitive dHL-60 cells. HL-60 cells were differentiated with TPA for $18 \mathrm{~h}$, then co-cultured with $P$. aeruginosa and stained for $P$. aeruginosa (blue) relative to LE-associated Rac or IQGAP1 (both green) and actin (red). Images show LE properties underlying $P$. aeruginosa binding and are representative of staining patterns obtained from two or more independent experiments. Bars, $10 \mu \mathrm{m}$. 
HT-29 cell model - HT-29 cells provided a means of monitoring alterations in LE architecture in relation to loss of $\mathrm{Pa}$-T3S sensitivity following treatments with $\mathrm{M} \beta \mathrm{CD}$ or PFO. To relate LE architecture to previous Pa-T3S sensitivity studies, HT-29 cells were treated with increasing concentrations of M $\beta C D$ or PFO, then co-cultured with Pa-ExoS-HA, and stained for Rac1 or PI3K in relation to cortical actin. Similar studies were performed using $P$. aeruginosa expressing an ExoS-GAP/ADPRT mutant to assess how ExoS activity influenced these studies.

HT-29 cells maintain properties of normal epithelial cells, including the ability to undergo collective migration as occurs during wound healing (145). Consistent with these properties, LE migration of HT-29 cells occurred at the periphery of cell clusters (Supplementary Fig. S5, 0 $M \beta C D$ or PFO). In monitoring the effects of $M \beta C D$ or PFO on LE properties in the presence of Pa-ExoS-HA, intact LE properties were evident at sub-T3S inhibitory concentrations of M $\beta C D$ $(0.5 \mathrm{mM})$ or PFO $\left(0.02 \mu \mathrm{g} \mathrm{ml}^{-1}\right)$, as observed by the close underlying association of Rac1 or PI3K with cortical actin in the direction of cell migration (Fig. S5, marked by arrowheads). Alterations in LE architecture became apparent in some cells at $1.0 \mathrm{mM} \mathrm{M} \beta C D$ or $0.1 \mu \mathrm{g} P \mathrm{P}$ $\mathrm{ml}^{-1}$ by the disruption or loss of actin staining relative to Rac1 or PI3K (Supplementary Fig. S5, marked by arrows). Extensive loss of LE actin became apparent at T3S inhibitory concentrations of $\mathrm{M} \beta C D(3 \mathrm{mM})$ or PFO $\left(0.2 \mu \mathrm{g} \mathrm{ml}^{-1}\right)$. To summarize, studies using $\mathrm{M} \beta \mathrm{CD}$ or PFO to inhibit T3S sensitivity found disruption of LE actin to closely coincide with interference of Pa-T3S.

Inhibitory effects of $\mathrm{M} \beta \mathrm{CD}$ or PFO on Pa-T3S also coincided with alterations in LE properties when cells were co-cultured with $P$. aeruginosa expressing enzymatically inactive ExoS. However, consistent with ExoS targeting LE associated Rac1 and Cdc42 (95, 233, 264), phenotypic alterations occurring in association with enzymatically inactive ExoS were different. Rather than observing severe disruption or loss of LE actin upon inhibition of Pa-T3S, LE 
protein architecture underlying actin was not disrupted in M $\beta C D$ or PFO treated HT-29 cells in association with $P$. aeruginosa expression of an ExoS-GAP/ADPRT mutant (results not shown). These results are of interest because they highlight the potential for T3S effectors, such as ExoS, to target host cell properties predicted to facilitate T3S translocon function. The effect of ExoS on LE actin also provides an explanation for why inhibitory effects of M $\beta C D$ and PFO on Pa-T3S were augmented by ExoS.

In examining $P$. aeruginosa association with LE properties in HT-29 cells, LE architecture was found to underlie P. aeruginosa binding in $54 \%$ of bacterial-HT-29 cell associations under conditions of T3S sensitivity. (Frequencies of $P$. aeruginosa binding to HT-29 cells were determined based on eight independent co-culture experiments and greater than $500 P$. aeruginosa-HT-29 cell enumerated associations.) This association is represented in Fig. 13 (T3S-sensitive panels), where IQGAP1, actin, and $P$. aeruginosa staining were used to visualize $P$. aeruginosa binding to the LE of HT-29 cells. $P$. aeruginosa did not preferentially associate with the LE of HT-29 cells after inhibition of T3S by treatment with $0.2 \mu \mathrm{g} \mathrm{PFO} \mathrm{ml}{ }^{-1}$, but rather P. aeruginosa binding became more random and occurred at intercellular junctions (Fig. 13, T3S-resistant panel). 

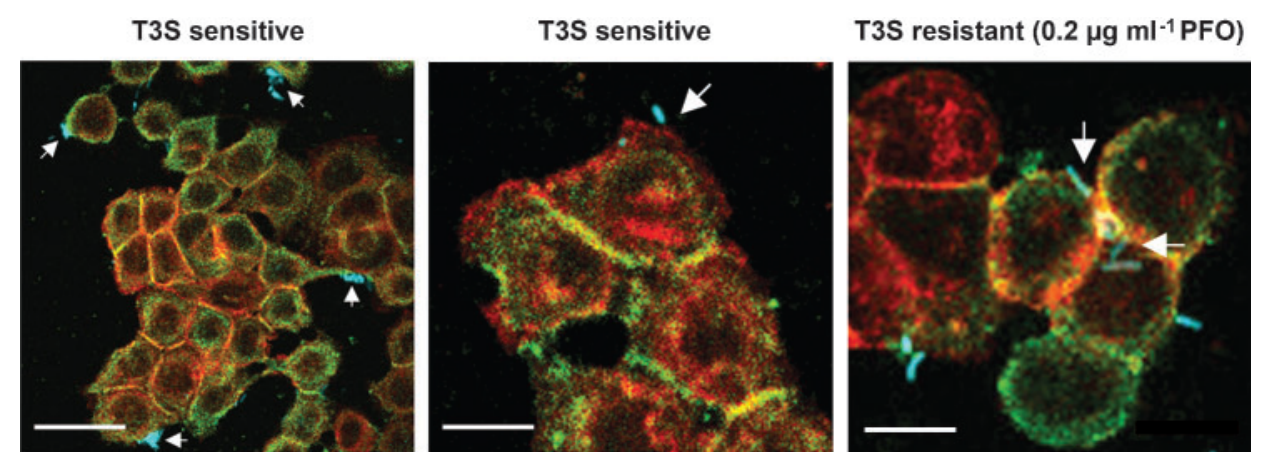

Figure 13. $P$. aeruginosa binding to LE of HT-29 cells. $P$. aeruginosa binding to T3S sensitive or T3S resistant HT-29 cells was assessed following co-culture with Pa-ExoS-HA and staining for IQGAP1 (green), actin (red), and P. aeruginosa (blue). Left panel (lower magnification) shows the frequency of $P$. aeruginosa association with the LE of clusters of T3Ssensitive HT-29 cells. Bar, $20 \mu \mathrm{M}$. Middle panel (higher magnification) shows $P$. aeruginosa association with LE lamellipodia. Right image shows the more random and intercellular association of $P$. aeruginosa with HT-29 cells treated with $0.2 \mu \mathrm{g} \mathrm{PFO} \mathrm{ml}^{-1}$ to cause loss of T3S sensitivity. Results are representative images obtained in two-three independent experiments. Bars, $10 \mu \mathrm{m}$. 
To summarize, studies examining the relationship between LE architecture and Pa-T3S sensitivity found that acquisition of LE architecture coincided with induction of Pa-T3S sensitivity in HL-60 cells. Conversely, disruption of LE architecture coincided with loss of Pa-T3S sensitivity in HT-29 cells. LE architecture was also found to underlie $P$. aeruginosa binding in both HL-60 and HT-29 cells under conditions of Pa-T3S sensitivity. As bacterial binding to host cells is integral to T3S translocon function, the preferential binding of $P$. aeruginosa to the LE of HL-60 and HT-29 cells under conditions of T3S sensitivity is consistent with the notion that the LE region serves as the host cell site of $P$. aeruginosa binding during T3S translocon insertion and function. Together these findings provide evidence of a close relationship or a role of eukaryotic cell LE properties in Pa-T3S sensitivity. 


\section{DISCUSSION}

Studies support the suggestion that T3S effectors are translocated into eukaryotic cells through a bacterially produced pore or channel in host cell membranes. Once internalized T3S effectors manipulate eukaryotic cell function in a manner that contributes to the establishment of infections. The purpose of our studies was to gain insight into the possible role of host cell properties in T3S translocon function and effector translocation. We chose $P$. aeruginosa as a model system to study this problem, because as an opportunistic pathogen, eukaryotic cell lines that are sensitive (HT-29 cells) or resistant (HL-60 cells) to Pa-T3S were available for mechanistic comparisons $(206,271)$. Addressing how host cells function in Pa-T3S required a series of progressive studies, but eventually studies of HT-29 and HL-60 cells converged to reveal a relationship between host cell LE properties and $\mathrm{Pa}-\mathrm{T} 3 \mathrm{~S}$ translocon function.

When T3S-sensitive HT-29 cells were used to study host cell properties involved in Pa-T3S, it proved experimentally difficult to inhibit $\mathrm{Pa}-\mathrm{T} 3 \mathrm{~S}$. Of the many agents tested, only $\mathrm{M} \beta \mathrm{CD}$ and PFO were found to inhibit Pa-T3S sensitivity in the absence of effects on $P$. aeruginosa. M $\beta C D$ and PFO have the common property of modulating membrane properties in a cholesteroldependent manner. However, in examining the mechanism underlying their inhibition of T3S, neither modulation of membrane cholesterol or lipid rafts appeared sufficient to inhibit Pa-T3S sensitivity. Rather, the eukaryotic cell property found to most closely correlate with loss of $\mathrm{Pa}$ T3S sensitivity was loss of HT-29 cell adherence to tissue culture wells.

Promyelocytic HL-60 cells are non-adherent and lack directional polarity and, along with polarized epithelial monolayers, were the only other cell line identified to be resistant to Pa-T3S (271). HL-60 cells can be experimentally induced to become Pa-T3S sensitive by treatment with the differentiating agent, TPA (271). TPA differentiated HL-60 cells also serve as a model system to study neutrophil migration in response to chemoattractants $(128,289)$. In using HL-60 
cells to study alterations in cellular properties that occur upon induction of Pa-T3S sensitivity, again a direct correlation was observed between HL-60 cell adherence to tissue culture wells and Pa-T3S sensitivity. It was further recognized in HL-60 cells that resistance to Pa-T3S related to the lack of PopB and PopD translocon protein insertion into the cellular membrane.

Recognition of a direct correlation between eukaryotic cell adherence mechanisms and Pa-T3S translocon function provided an important missing link in understanding host cell involvement in Pa-T3S, and led us to explore the functional relationship between eukaryotic cell adhesion mechanisms and Pa-T3S. Focal complexes form dynamic cell adherence structures at the LE and can be distinguished from more stable focal adhesions based on Rac/Cdc42, rather than Rho, mediating their adherence mechanisms. In using Rac1 or Cdc42 inhibitors to examine the role of LE focal complexes in T3S, both agents were found to inhibit T3S effector translocation. This was in contrast with ROCK or Src kinase inhibitors that target focal adhesions, which caused no significant inhibition of Pa-T3S. Notably, Rac1 and Cdc42 inhibitors were more efficient in inhibiting Pa-T3S if added prior to adherence of HT-29 cells to tissue culture wells. Together these studies provided evidence that LE adherence properties were facilitating and stabilizing Pa-T3S effector translocation.

The relationship between eukaryotic cell LE properties and Pa-T3S sensitivity was corroborated in studies of HL-60 cells. SHL-60 cells gained sensitivity to Pa-T3S in culture after multiple passages but remained phenotypically indistinguishable from rHL-60 cells relative to a round, non-adherent morphology. In comparing LE properties of rHL-60 and sHL-60 cells, differences observed in sHL-60 cells included enhanced MT projections, Rac co-localization with actin, and the polarized localization of PI3K. These observations are consistent with sHL-60 cells acquiring LE architecture in conjunction with induction of $\mathrm{Pa}$-T3S sensitivity. 
As an opportunistic pathogen, $P$. aeruginosa infects disrupted epithelial barriers, whereas intact epithelial barriers that maintain apical-basolateral polarity are resistant to $P$. aeruginosa infections $(91,156,243)$. Evidence that host cell LE properties that direct cell migration function in Pa-T3S sensitivity is highly consistent with $P$. aeruginosa infections, which target disrupted epithelial barriers undergoing wound-healing cell migration. In migrating epithelial cells, polarity is mediated by interlinked feedback loops that involve Rac, Cdc42, Rho, PI3K, integrins, and MTs. In examining host cell proteins contributing to Pa-T3S sensitivity, a hierarchal order of protein function was recognized with first, actin, Rac1, and Cdc42, then MTs and IQGAP1, and to a lesser degree PI3K functioning in Pa-T3S. Notably the hierarchal order of proteins affecting Pa-T3S sensitivity paralleled the functional assembly of cellular proteins at the LE. Because of the dependence of the LE on protein assembly, LE function was examined by IF staining and microscopic analysis of LE architecture. In using both HL-60 and HT-29 cell models to examine the relationship between LE cell architecture and Pa-T3S, the integrity of LE architecture closely coincided with Pa-T3S sensitivity. Collectively, identification of: (1) a relationship between host cell adherence mechanisms and Pa-T3S sensitivity; (2) evidence for a role of focal complexes but not focal adhesions in Pa-T3S; (3) a hierarchal order of protein involvement in Pa-T3S function that reflected LE structure; and (4) the close correlation between LE cell architecture and Pa-T3S sensitivity all point to eukaryotic cell LE properties playing a role in sensitivity to PaT3S.

Eukaryotic membranes are structured to resist channel formation as occurs during T3S but can be modulated through localized alterations in membrane composition or mechanical forces to favor channel formation. Pa-T3S translocon formation in eukaryotic membranes requires translocator proteins, PopB, PopD, and PcrV. Beyond this, a role of host cell properties in PaT3S became apparent from the identification of Pa-T3S-resistant cells $(206,271)$. Studies presented here provide evidence and lead us to hypothesize that LE properties are the host cell 
factor required for Pa-T3S translocon insertion and effector translocation. Mechanistically, the role of LE properties in facilitating T3S translocon membrane insertion can be explained by alterations in membrane properties that occur at the LE. As shown in the model of host cell involvement in $\mathrm{Pa}$-T3S in Fig. 14, activated Rac1/Cdc42-mediated actin polymerization at the LE drives cell membrane lamellipodia or filopodia protrusions, which introduce forces on plasma membranes that could facilitate PopB/PopD membrane insertion. Actin filaments must be tethered to the cell membrane to exert forces that drive membrane protrusions, and LE adherence mechanisms secure and stabilize these actin-membrane associations. The relationship between host cell adherence and Pa-T3S can be explained by the stabilizing role that LE adherence processes have on actin-membrane associations predicted to facilitate translocon insertion. The subtlety of alterations in cell architecture required for Pa-T3S sensitivity became evident in SHL-60 cells, in which induction of MT radiations and polarized colocalization of Rac with actin were observed to coincide with induction of Pa-T3S sensitivity. Once T3S is established, host cell properties involved in Pa-T3S translocon function appear quite stable as evidenced by the requirement of agents, such as $M \beta C D$ and PFO, which disrupt cell polarity and LE architecture to inhibit Pa-T3S sensitivity. Retrospectively, the inability of most agents in Supplementary Table SI to inhibit Pa-T3S can be explained by their inability to cause severe alterations in cell polarity. The notion that $M \beta C D$ and PFO interfere with Pa-T3S by disrupting cell polarity and LE architecture is consistent with recent studies which found $M \beta C D$ to alter the polarized redistribution of cellular factors mediating cell migration (267). Cholesterol is known to accumulate at the LE of migrating cells, where it has been hypothesized to alter plasma membrane viscosity to allow the dynamic actin network to push the membrane forward (193). The correlation between inhibition of Pa-T3S sensitivity by M $\beta C D$ and PFO with loss of LE structure attests to the possibility that the function of membrane cholesterol in PaT3S relates to its polarization to the LE and its facilitation of actin-induced membrane 
protrusions. Important to an understanding of host cell involvement in Pa-T3S is that loss or gain of LE architecture in HT-29 or HL-60 cells provides an explanation for alterations in Pa-T3S sensitivity and for the stabilizing role of LE adherence in translocon function. 


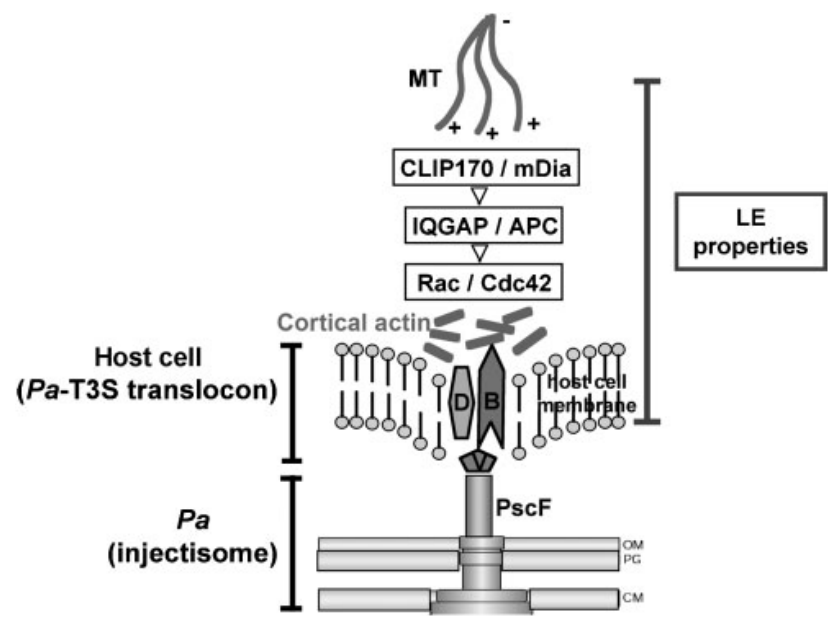

Figure 14. Model for the role of eukaryotic cell properties in Pa-T3S. Our model predicts that alterations in membrane properties associated with F-actin polymerization at the LE induce forces on membranes that are required for PopB (B) and PopD (D) translocon insertion and function. V, PcrV, (PscF) T3S needle protein. The role of specific leading edge proteins in PaT3S is described in the text. Pa, P. aeruginosa. 
The opportunistic nature of $P$. aeruginosa infections and reliance on a predisposing host cell factor were essential to our identifying host cell properties involved Pa-T3S. However, the opportunistic nature of $P$. aeruginosa infections likely adds a dimension to T3S translocon function not apparent in obligate Gram-negative pathogens. For example, our model predicts that cortical actin polymerization at the LE is required for Pa-T3S translocon function, which closely aligns with recent studies in Yersinia that have found T3S translocon function to also rely on actin polymerization (207). Yersinia is known to engage host cell integrin receptors in association with T3S effector translocation, and this leads to Rho activation and actin polymerization, which are proposed to trigger YopB/YopD translocon function. $P$. aeruginosa as an opportunistic pathogen is unable to engage integrin or other actin polymerization mechanisms, and as a result is dependent on LE properties that trigger actin polymerization for the PopB/PopD translocon function. The role of actin polymerization in Yersinia T3S, and our studies which identify a role of actin polymerization in Pa-T3S, draw attention to a potential central role of host cell cortical actin in T3S translocon function.

To summarize, the complexity of bacterial-eukaryotic cell interactions during T3S has made it difficult to understand how eukaryotic cell properties might contribute to T3S translocon function. By using cell culture models that are sensitive or resistant to Pa-T3S we were able to identify a relationship between LE properties and host cell Pa-T3S sensitivity, and this has led us to hypothesize that cortical actin-induced alterations in membrane properties at the LE facilitate T3S translocon insertion and function. The integral and complex role of LE properties in eukaryotic cell survival attests to the highly co-evolved nature of $\mathrm{Pa}$-T3S relative to eukaryotic cell function. The use of the LE in Pa-T3S is highly consistent with the opportunistic lifestyle of $P$. aeruginosa, which targets wounded epithelial barriers undergoing cell migration. The requirement of LE properties for Pa-T3S is predicted to differ from that of obligate pathogens, such as Yersinia, which are able to engage integrin to induce actin polymerization required for 
translocon function. The ability of cortical actin dynamics to alter membrane properties to favor T3S translocon insertion or function has the potential to be a common theme for host cell involvement in T3S. The precise characterization of host involvement in T3S is anticipated to provide insight into the design and development of novel therapeutic approaches to inhibit this stage in the infectious process of Gram-negative pathogens. 


\section{ACKNOWLEDGMENTS}

We wish to thank Dara Frank and Joseph Barbieri (Medical College of Wisconsin) for providing P. aeruginosa strains, the ExoS-HA construct, and PopB and PopD antibodies. We also wish to thank Rodney Tweten (University of Oklahoma Health Sciences Center) for providing PFO and PFO mutants, and Thomas Kirchhausen (Harvard Medical School) and Gerald B. Hammond (University of Louisville) for providing secramine A. We would also like to acknowledge the technical assistance of Jennifer Salazar and Yeremie Canizales in these studies, and the statistical assistance of Gerry Hobbs, West Virginia University (WVU) Department of Statistics. All IF images were taken at the WVU Imaging Facility with the assistance of Karen Martin. These studies were supported by Public Health Services Grant NIH NIAID 45569, by the Mary C. Babb Cancer Center Foundation Fund 2V882, and by the WVU HSC internal grants funding program. 
Supplementary Table S1. Pa-T3S drug treatments

All drugs were tested at their reported $\mathrm{IC}_{50}$ or greater, but positive controls for each drug activity were not performed.

\begin{tabular}{|c|c|c|c|}
\hline Drug (concentration) & Drug mechanism & Effect on $\mathrm{T}_{3} \mathrm{~S}^{1}$ & Effect on $P$. aeruginos $a^{2}$ \\
\hline \multicolumn{4}{|l|}{ I. Membrane modulation: } \\
\hline \multicolumn{4}{|l|}{ General } \\
\hline Methanol $(0.5-5 \%)^{3}$ & Membrane & No effect & $\mathrm{ND}^{16}$ \\
\hline & dehydrating/permeabilizing & & \\
\hline Ethanol $(0.5-5 \%)^{3}$ & Membrane & No effect & ND \\
\hline & dehydrating/permeabilizing & & \\
\hline Formaldelyde $(1-2 \%)^{4}$ & $\begin{array}{c}\text { Cross-linking } \\
\text { agent/Fixative/Cytotoxic }\end{array}$ & $\underline{\text { Inhibits }}$ & Drug removed \\
\hline Lipid rafts & & & \\
\hline $\begin{array}{l}\text { Methyl } \beta \text {-cyclodextrin ( } 3 \\
\mathrm{mM})^{5}\end{array}$ & $\begin{array}{c}\text { Abstracts membrane } \\
\text { cholesterol }\end{array}$ & $\underline{\text { Inhibits }}$ & No effect \\
\hline Filipin $(7-10 \mu \mathrm{M})^{5}$ & $\begin{array}{c}\text { Sequesters membrane } \\
\text { cholesterol }\end{array}$ & Inhibits & Affects $P$. aeruginosa $(\mathrm{G})$ \\
\hline $\begin{array}{l}\text { Anti-ceramide }(0.5-4 \mu \mathrm{g} \\
\left.\mathrm{ml}^{-1}\right)^{6}\end{array}$ & Abrogates CD95 clustering & No effect & ND \\
\hline Imipramine $(25-200 \mu \mathrm{M})^{7}$ & $\begin{array}{c}\text { Acid sphingomyelinase } \\
\text { inhibitor }\end{array}$ & No effect & ND \\
\hline Receptors & & & \\
\hline $\begin{array}{l}\text { Neuraminidase }(50-400 \\
\mathrm{mM})^{6}\end{array}$ & $\begin{array}{l}\text { Cleaves sialic acid- } \\
\text { glycosidic bonds }\end{array}$ & No effect & ND \\
\hline Endoglycosidase $(20 \mathrm{nM})^{5}$ & $\begin{array}{l}\text { Cleaves N-linked } \\
\text { oligosaccharides }\end{array}$ & No effect & ND \\
\hline $\begin{array}{l}\text { PNGase F }(10-200 \mathrm{mU} \\
\left.\mathrm{ml}^{-1}\right)^{6}\end{array}$ & $\begin{array}{l}\text { Cleaves N-linked } \\
\text { oligosaccharides }\end{array}$ & No effect & ND \\
\hline Tunicamycin $(2 \mu \mathrm{M})^{8}$ & $\begin{array}{l}\text { Inhibits N-linked } \\
\text { glycosylation }\end{array}$ & No effect & ND \\
\hline
\end{tabular}




\begin{tabular}{|c|c|c|c|}
\hline Drug (concentration) & Drug mechanism & Effect on $T 3 S^{1}$ & Effect on $P$. aeruginos ${ }^{2}$ \\
\hline $\begin{array}{l}\text { Benzyl-alpha GalNAc (2 } \\
\mathrm{mM})^{9}\end{array}$ & $\begin{array}{l}\text { Inhibits O-linked } \\
\text { glycosylation }\end{array}$ & No effect & ND \\
\hline $\begin{array}{l}\text { PI-phospholipase } \mathrm{C}(0.05- \\
\left.1.5 \mathrm{U} \mathrm{ml}^{-1}\right)^{6}\end{array}$ & $\begin{array}{l}\text { Cleaves GPI-linked } \\
\text { glycoproteins }\end{array}$ & No effect & ND \\
\hline Trypsin $(0.05 \%)^{10}$ & $\begin{array}{l}\text { Serine protease (limited } \\
\text { specificity) }\end{array}$ & No effect & ND \\
\hline $\begin{array}{l}\text { Proteinase K }(0.1-1.0 \mu \mathrm{g} \\
\left.\mathrm{ml}^{-1}\right)^{10}\end{array}$ & $\begin{array}{l}\text { Serine protease (broad } \\
\text { specificity) }\end{array}$ & Inhibits & Affects $P$. aeruginosa $(\mathrm{E})$ \\
\hline Protease inhibitors $^{6}$ & $\begin{array}{l}\text { Aprotinin/Leupeptin/Bestat } \\
\text { in/Pepstatin A/E-64 }\end{array}$ & No effect & ND \\
\hline $\operatorname{AEBSF}(200 \mu \mathrm{M})^{6}$ & Serine protease inhibitor & Inhibits & Affects $P$. aeruginosa $(\mathrm{E})$ \\
\hline $\begin{array}{l}\text { Cycloheximide }(10 \mu \mathrm{g} \\
\left.\mathrm{ml}^{-1}\right)^{11}\end{array}$ & $\begin{array}{c}\text { Inhibits protein } \\
\text { synthesis/receptor recycling }\end{array}$ & No effect & ND \\
\hline Membrane pores & & & \\
\hline $\begin{array}{l}\text { Streptolysin } \mathrm{O}(0.5 \mathrm{U} \\
\left.\mathrm{ml}^{-1}\right)^{6}\end{array}$ & $\begin{array}{c}\text { Binds cholesterol/Forms } 30 \\
\text { nm membrane pores }\end{array}$ & Inhibits & Affects $P$. aeruginosa $(\mathrm{E}, \mathrm{A})$ \\
\hline $\begin{array}{l}\text { Perfringolysin } \mathrm{O}(0.1 \mu \mathrm{g} \\
\left.\mathrm{ml}^{-1}\right)^{6}\end{array}$ & $\begin{array}{l}\text { Binds cholesterol/Forms } \\
30-45 \mathrm{~nm} \text { membrane pores }\end{array}$ & Inhibits & No effect \\
\hline Ion channels & & & \\
\hline SKF96365 $(40-200 \mu \mathrm{M})^{5}$ & $\begin{array}{c}\text { Voltage gated } \mathrm{Ca}^{2+} \text { channel } \\
\text { blocker }\end{array}$ & No effect & ND \\
\hline Calcimycin $(1-6 \mu \mathrm{M})^{5}$ & $\begin{array}{l}\text { Ionophore/transports } \mathrm{Ca}^{2+} \\
\text { across plasma membrane }\end{array}$ & No effect & ND \\
\hline Ionomycin $(0.5-50 \mu \mathrm{M})^{6}$ & $\begin{array}{l}\text { Ionophore/transports } \mathrm{Ca}^{2+} \\
\text { from intracellular stores }\end{array}$ & No effect & ND \\
\hline $\begin{array}{l}\text { ii) Cytoskeletal } \\
\text { modulation: }\end{array}$ & & & \\
\hline Microtubules & & & \\
\hline Colchicine $(0.6-10)^{6}$ & $\begin{array}{l}\text { Disrupts tubulin } \\
\text { polymerization }\end{array}$ & No effect & ND \\
\hline Nocodazole $(133 \mathrm{nM})^{6}$ & $\begin{array}{l}\text { Promotes tubulin } \\
\text { depolymerization }\end{array}$ & No effect & ND \\
\hline
\end{tabular}




\begin{tabular}{|c|c|c|c|}
\hline Drug (concentration) & Drug mechanism & Effect on T3S ${ }^{1}$ & Effect on $P$. aeruginos $a^{2}$ \\
\hline Taxol $(1 \mu \mathrm{M})^{6}$ & Stabilizes microtubules & No effect & ND \\
\hline \multicolumn{4}{|l|}{ Actin } \\
\hline Cytochalasin D (0.5-40 & Inhibits actin & No effect & ND \\
\hline$\mu \mathrm{M})^{6}$ & polymerization & & \\
\hline Jasplakinolide $(150 \mathrm{nM})^{6}$ & Stabilizes/reorganizes actin & No effect & ND \\
\hline \multicolumn{4}{|l|}{ iii) Cell process } \\
\hline \multicolumn{4}{|l|}{$\underline{\text { modulation }}$} \\
\hline \multicolumn{4}{|l|}{ Signalling } \\
\hline Wortmannin $(0.5-10 \mu \mathrm{M})^{6}$ & $\begin{array}{l}\text { PI3K inhibitor (all } \\
\text { isoforms) }\end{array}$ & No effect & ND \\
\hline LY294002 $(2-100 \mu \mathrm{M})^{6}$ & $\begin{array}{c}\text { PI3K inhibitor (all } \\
\text { isoforms) }\end{array}$ & No effect & ND \\
\hline Genistein $(200-800 \mu \mathrm{M})^{11}$ & Protein Tyr kinase inhibitor & No effect & ND \\
\hline \multirow[t]{2}{*}{ PPI $(1-100 \mu \mathrm{M})^{6}$} & Src-family protein $\mathrm{Tyr}$ & No effect & ND \\
\hline & kinase inhibitor & & \\
\hline \multirow[t]{2}{*}{ PP2 $(1-100 \mu \mathrm{M})^{6}$} & Src-family protein $\mathrm{Tyr}$ & No effect & ND \\
\hline & kinase inhibitor & & \\
\hline Bisindolymaleimide (20- & Protein kinase $C \beta /$ Ser-Thr & No effect & ND \\
\hline $1000 \mathrm{nM})^{12}$ & kinase inhibitor & & \\
\hline \multirow[t]{2}{*}{ SB $202190(2 \mu \mathrm{M})^{7}$} & P38 MAP/Ser-Thr kinase & No effect & ND \\
\hline & inhibitor & & \\
\hline \multirow[t]{2}{*}{$\operatorname{MAFP}(1-10 \mu \mathrm{M})^{7}$} & Cytosolic phospholipase & No effect & ND \\
\hline & A2 inhibitor & & \\
\hline W7 $(50-100 \mu \mathrm{M})^{6}$ & $\mathrm{Ca}^{2+} /$ calmodulin inhibitor & No effect & ND \\
\hline \multicolumn{4}{|l|}{ Trafficking } \\
\hline Brefeldin A $(0.5-20 \mu \mathrm{g}$ & Disrupts ER to golgi & No effect & ND \\
\hline $\left.\mathrm{ml}^{-1}\right)^{5}$ & trafficking & & \\
\hline EHNA $(0.5-100 \mu \mathrm{M})^{12}$ & Adenosine deaminase & No effect & ND \\
\hline & inhibitor (Dynein inhibitor) & & \\
\hline \multicolumn{4}{|l|}{ Energy } \\
\hline Deoxyglucose (25 & Depletes ATP/GTP & No effect & Drug removed \\
\hline $\mathrm{mM}) / \mathrm{NaN}_{3}(25 \mathrm{mM})^{13}$ & & & \\
\hline
\end{tabular}




\begin{tabular}{|lccc|}
\hline \multicolumn{1}{|c}{ Drug (concentration) } & Drug mechanism & Effect on T3S & Effect on P. aeruginosa $^{\mathbf{1}}$ \\
\hline Ouabain $(10 \mu \mathrm{M})^{14}$ & Blocks Na $^{+} / \mathrm{K}^{+}$ATPase & No effect & Drug removed \\
Cyanide $(\mathrm{CCCP})(10 \mu \mathrm{M})^{15}$ & $\begin{array}{c}\text { Inhibits electron transport } \\
\text { chain }\end{array}$ & No effect & Drug removed \\
\hline
\end{tabular}

${ }^{1}$ Studies were performed with $P$. aeruginosa T3S-sensitive, HT-29 and/or T24 epithelial cells. T3S was determined based on ExoS translocation and ADP-ribosylation of RalA.

${ }^{2}$ Effect on $P$. aeruginosa: $\mathrm{G}$, inhibits $P$. aeruginosa growth; E, decreases T3S effector secretion; A, interferes with $P$. aeruginos $a$ adherence to host cells.

${ }^{3}$ Cells pretreated with drug for $15 \mathrm{~min}$ and drug either removed or present during co-culture.

${ }^{4}$ Cells pretreated with drug for $15 \mathrm{~min}$, drug not present during co-culture.

${ }^{5}$ Cells pretreated with drug for $30 \mathrm{~min}$, drug present during co-culture.

${ }^{6}$ Cells pretreated with drug for $1 \mathrm{~h}$, drug present during co-culture.

${ }^{7}$ Cells pretreated with drug for $15 \mathrm{~min}$, drug present during co-culture.

${ }^{8}$ Cells pretreated with drug for $3 \mathrm{~h}$, drug present during co-culture.

${ }^{9}$ Cells pretreated with drug for $16 \mathrm{~h}$, drug present during co-culture.

${ }^{10} \mathrm{Cells}$ pretreated with protease for $15 \mathrm{~min}$, protease inhibitor added, cells recovered, resuspended in fresh medium and replated.

${ }^{11}$ Cells pretreated with drug for $45 \mathrm{~min}$ and drug present during co-culture.

${ }^{12}$ Cells pretreated with drug for $30 \mathrm{~min}$, drug not present during co-culture.

${ }^{13}$ Cells pretreated with drug for $4 \mathrm{~h}$, drug not present during co-culture.

${ }^{14}$ Cells pretreated with drug for $45 \mathrm{~min}$, drug not present during co-culture.

${ }^{15}$ Cells pretreated with drug for $1 \mathrm{~h}$, drug not present during co-culture.

${ }^{16} \mathrm{ND}$, not determined. 
a.

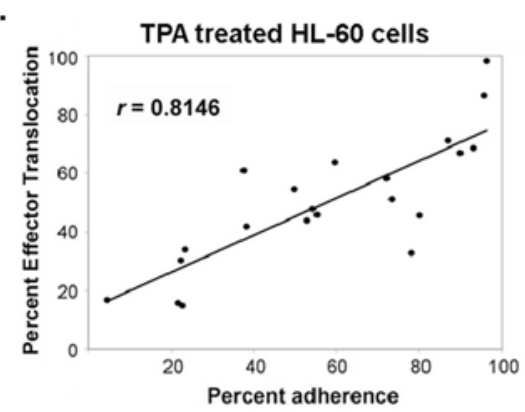

b.

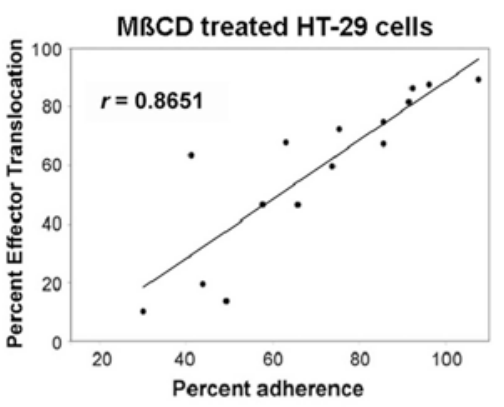

c.

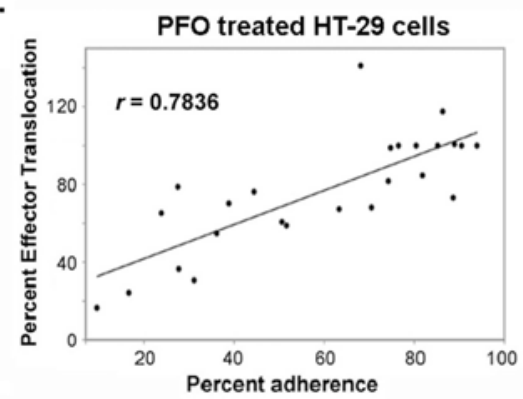

Supplementary Figure S1. Examining the relationship between eukaryotic cell adherence mechanisms and Pa-T3S sensitivity. Graphs showing the relationship between adherence to tissue culture wells and Pa-T3S sensitivity of individual samples for: (a) HL-60 cells, following TPA induction of T3S sensitivity, or for HT-29 cells upon loss of T3S sensitivity by treatment with (b) M $B C D$ or (c) PFO. Percentage adherence was calculated using trypan blue staining to enumerate adherent cells, which was related to adherent cells in control samples. Percentage effector translocation was calculated based on the efficiency of ExoS ADP ribosylation of host cell RalA, as determined by densitometry analysis of immunoblots comparing modified RalA to total RalA for individual samples. $r$, Pearson's correlation coefficient. Results from two or three independent experiments are represented for each graph. 
a.

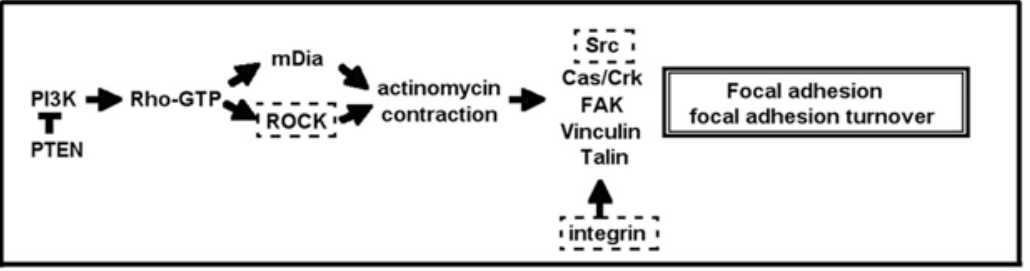

b.
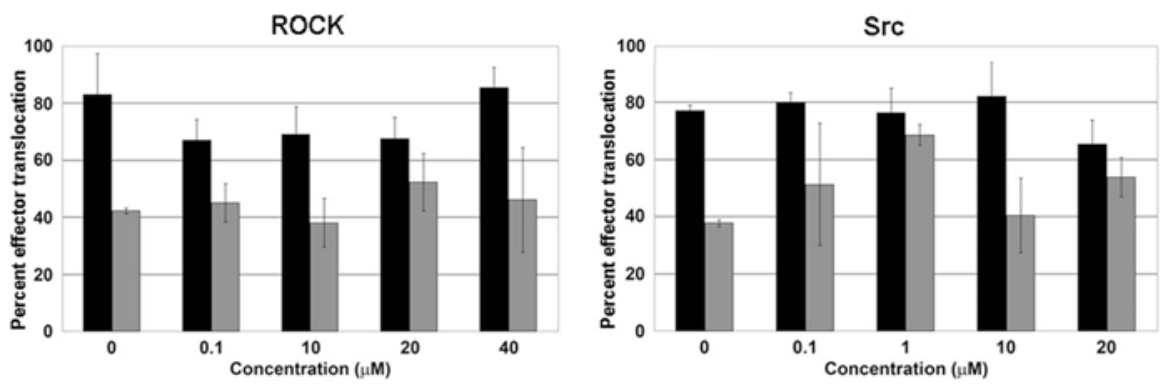

Supplementary Figure S2. Examining the role of focal adhesions in host cell Pa-T3S sensitivity. (a) Diagram of major eukaryotic cell proteins mediating focal adhesions. Proteins enclosed in dashed boxes were not found to mediate Pa-T3S sensitivity. (b) To examine the role of focal adhesions in Pa-T3S, HT-29 cells were treated with a ROCK inhibitor (Y-27632) or Src kinase inhibitor (PP2), either $48 \mathrm{~h}$ after adherence (black bars) or immediately following seeding prior to adherence (grey bars), and then co-cultured with $\mathrm{Pa}$-ExoS-HA in the presence of drugs. T3S sensitivity was quantified in cell lysates based on the efficiency of ExoS translocation and ADP ribosylation of RalA. Drug concentration relative to efficiency of Pa-T3S effector translocation is presented, and results are representative of three independent experiments. 


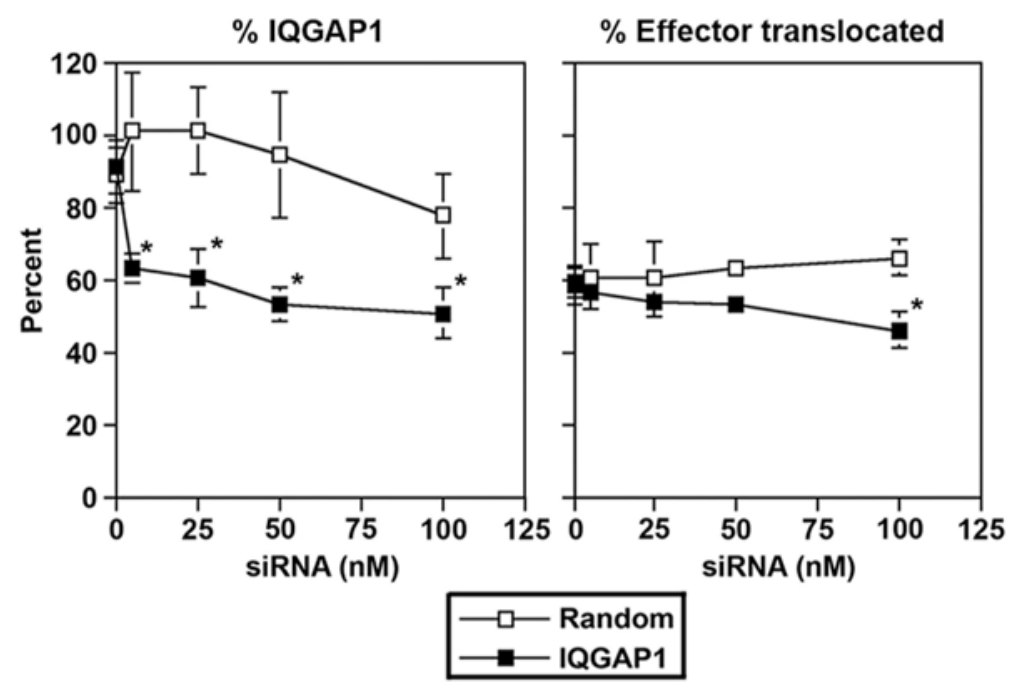

Supplementary Figure S3. Examining the role of leading edge IQGAP1 in host cell Pa-T3S sensitivity. HT-29 cells were transfected with IQGAP1 or random non-targeting siRNA. After a $72 \mathrm{~h}$ transfection period, adherent HT-29 cells were co-cultured with Pa-ExoS-HA. Cell lysates were assayed for IQGAP1 expression, using densitometry to quantify IQGAP1 immunoblots, and Pa-T3S effector translocation was quantified based on ExoS ADP ribosylation of RalA. The mean \pm SEM of percentage IQGAP1 or T3S effector translocation, when compared with nontransfected cells co-cultured with $\mathrm{Pa}-\mathrm{ExoS}-\mathrm{HA}$, is shown relative to increasing concentrations of siRNA. Results of three independent experiments are represented. Significant decreases $(P<0.001)$ in IQGAP1 or effector translocation relative to non-targeting siRNA are indicated by asterisks. 
a. rHL-60 cells

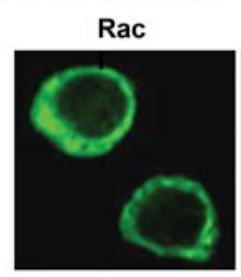

IQGAP1

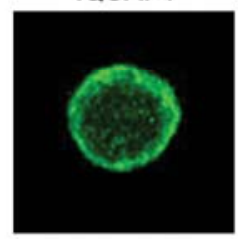

PI3K

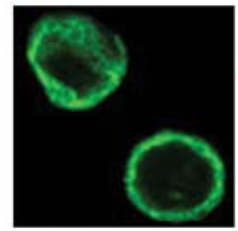

Rho

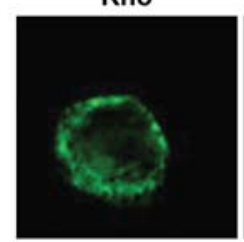

Actin

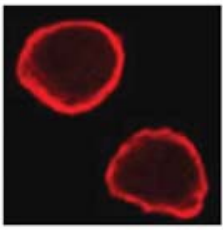

Actin

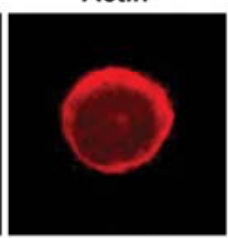

Actin

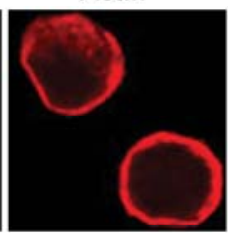

Actin

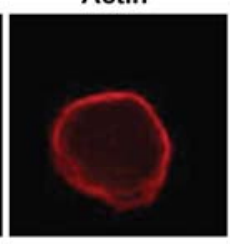

Merge

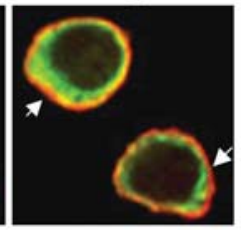

Merge

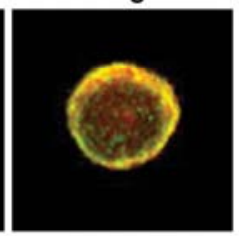

Merge

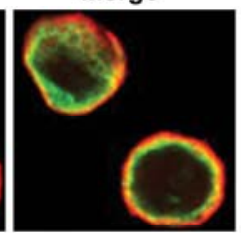

Merge

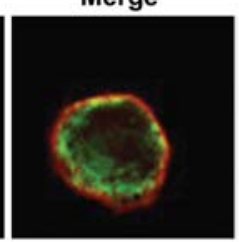

b. sHL-60 cells
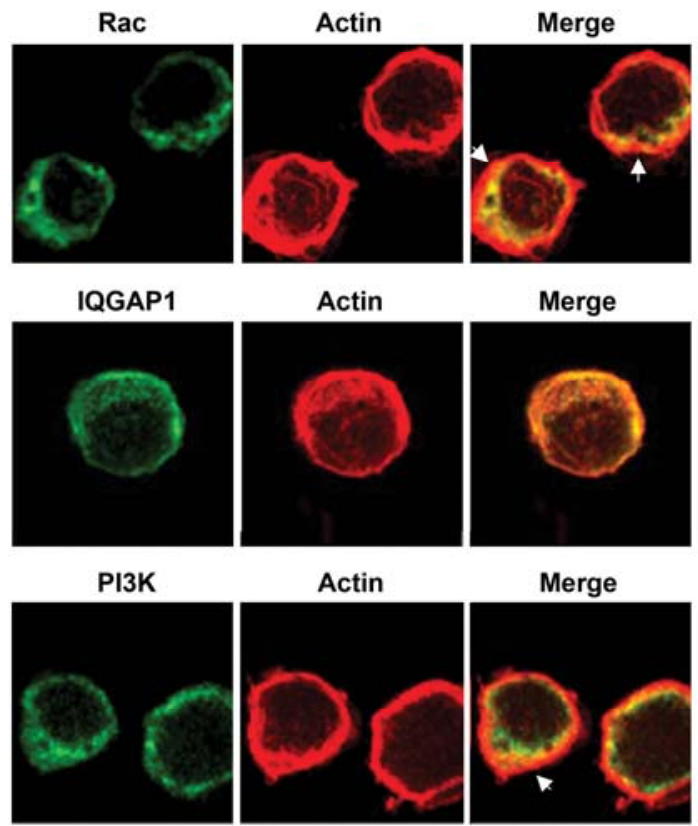

Actin
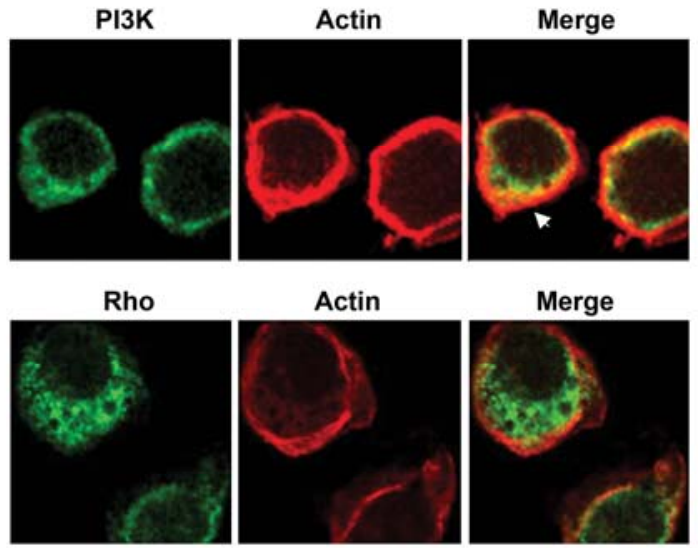

Actin

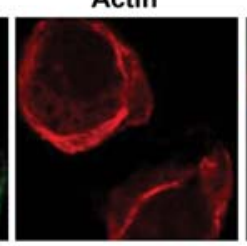

Merge

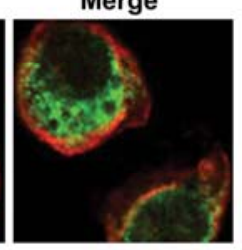

Supplementary Figure S4. Relationship between HL-60 cell LE architecture and Pa-T3S sensitivity. LE architecture of: (a) T3S-resistant rHL-60 cells, and (b) T3S-sensitive sHL-60 cells was compared by immunofluorescent staining for Rac, IQGAP1, and PI3K, with Rho serving as a focal adhesion marker. Proteins were visualized using Alexa Fluor-488 conjugated anti-mouse antibody (each stained green). Actin was stained in each panel with phalloidinTRITC (red). Co-localization with actin is indicated by yellow staining in merged images. Arrows point to the major differences in rHL-60 and sHL-60 cells, which are the co-localization of Rac1 with actin and increased polarization of PI3K in sHL-60 cells. Images are representative of the most frequent staining patterns, as observed in two independent experiments. 
Supplementary Figure S5. Relationship between HT-29 cell LE architecture and Pa-T3S sensitivity. HT-29 cells were treated with increasing concentrations of Pa-T3S inhibitors $(\mathbf{a}, \mathbf{c})$ $\mathrm{M} \beta C D$ or $(\mathbf{b}, \mathbf{d}) \mathrm{PFO}$, as described in the text, then co-cultured with Pa-ExoS-HA. Alterations in the localization of LE-associated Rac1 $(\mathbf{a}, \mathbf{b})$ or PI3K $(\mathbf{c}, \mathbf{d})$ (both stained green) were assessed relative to actin (red). P. aeruginosa were present in these studies but not stained. Arrowheads point to intact LE structures at 0 and sub-T3S inhibitory concentrations of M $\beta C D(0.5 \mathrm{mM})$ and PFO $\left(0.02 \mu \mathrm{g} \mathrm{ml}^{-1}\right)$. Arrows point to disrupted leading edge structures, as evident from the interruption or loss of cortical actin in cells treated with 1 or $3 \mathrm{mM} \mathrm{M \beta CD}$ or 0.1 or $0.2 \mu \mathrm{gl}^{-1}$ PFO. Results are representative of images obtained in two or three independent studies. 


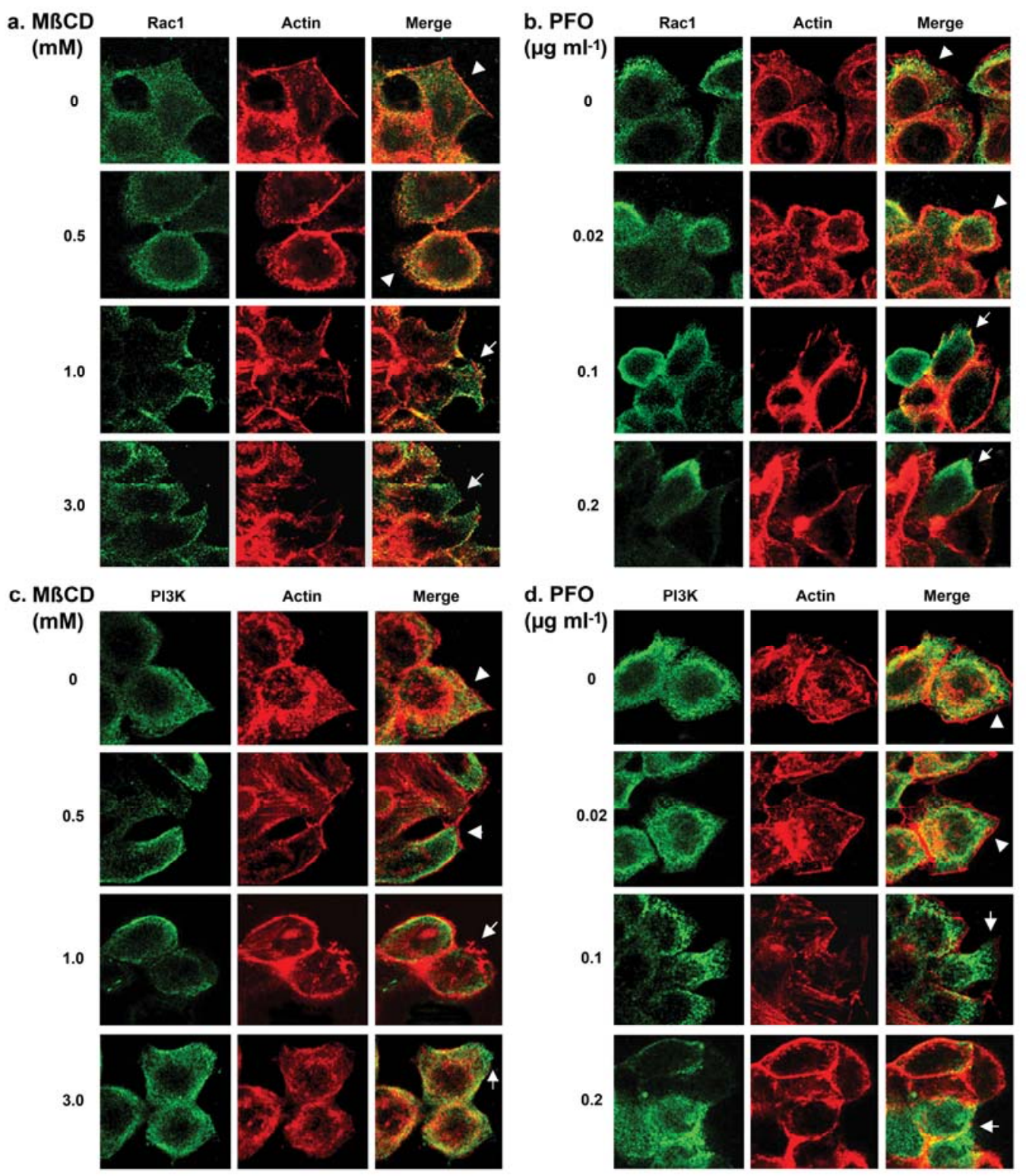


Chapter 3: Gaining Insight into the Opportunistic Nature of Pseudomonas aeruginosa Infections through Manipulations of the T3S Effector ExoS

Contents of this chapter have been submitted to Infection and Immunity and are currently under review The authors on the manuscript are:

Dacie R. Bridge, Karen H. Martin, Elizabeth R. Moore, Wendy M. Lee, James A. Carroll, Claudia L. Rocha, and Joan C. Olson. 


\section{ABSTRACT}

Opportunistic Pseudomonas aeruginosa $(\mathrm{Pa})$ infections target wounded epithelial barriers, but the property of wounded cells that increases susceptibility to $\mathrm{Pa}$ infections remains unclear. This study further explored the hypothesis that initiation of $\mathrm{Pa}$ infections is linked to cell migration properties induced during wound repair using: (1) highly migratory T24 epithelial cells as a cell culture model, (2) mutations in ExoS to manipulate type III secretion (T3S) involved in initiating $\mathrm{Pa}$ infections, and (3) visualization of ExoS and PopB translocation to monitor the $\mathrm{Pa}$ infectious process. In these analyses $\mathrm{Pa}$ expressing wild type ExoS bound preferentially to the leading edge of T24 cells, where translocation of ExoS GTPase activating protein (GAP) and ADPribosyltransferase (ADPRT) activities rapidly shutdown leading edge properties involved in cell migration in conjunction with inhibition of T3S. ExoS GAP inhibits $P a$ internalization, and an ExoS GAP- mutant increased T3S translocation in association with $P a$ internalization and secretion of ExoS within T24 cells. ExoS ADPRT activity disrupted actin anchoring to the plasma membrane and constricted leading edge architecture. Mutations in ExoS ADPRT activity in turn led to increased T3S translocation consistent with it targeting cellular proteins that inhibit T3S. An ExoS GAP-/ADPRT- mutant enhanced both Pa internalization and T3S translocation, and this occurred in conjunction with maintenance of leading edge architecture and the entry and guidance of $\mathrm{Pa}$ along actin filaments. ExoS was also found to track actin within T24 cells, providing a mechanism for the facilitated translocation of ExoS at the leading edge. Collectively, this study demonstrated the ability of: (1) $P a$ to secret ExoS intracellularly when ExoS GAP activity is mutated, introducing in novel means of 'translocating' ExoS into host cells; (2) ExoS ADPRT activity to target a cellular substrate(s) that disrupts actin-plasma membrane associations and leading edge architecture in conjunction with inhibition of ExoS translocation, and (3) both ExoS and $\mathrm{Pa}$ to interact or align with actin during the $\mathrm{Pa}$ infectious process. In conclusion, ExoS was found to contribute to the opportunistic nature of $\mathrm{Pa}$ infections through its ability to interrupt $P a$ internalization and T3S translocation to limit the $P a$ infectious process. 


\section{INTRODUCTION}

Pseudomonas aeruginosa $(\mathrm{Pa})$ is a ubiquitous environmental bacterium capable of causing infections of high morbidity and mortality in individuals who have damaged epithelial barriers, are immunocompromised, or have cystic fibrosis. While the cellular property that allows the establishment of $\mathrm{Pa}$ infections remains unknown, healthy epithelial monolayers with apical basolateral polarity are recognized to be resistant to $P a$ infections $(87,156,243)$. Pa maintains a broad range of virulence factors that facilitate its survival in nature and its pathogenicity in hosts. Of these, the type III secretion (T3S) system is conserved in both environmental and clinical $\mathrm{Pa}$ isolates (82) and contributes to the severity of acute $\mathrm{Pa}$ infections (129). T3S provides bacteria with a means of directly translocating effectors into eukaryotic cells via a bacterially formed needle-like conduit nanostructure and translocon channel in host cell membranes. Translocated effectors then allow bacteria to manipulate host cell processes in a manner specific to the lifestyle of the bacteria. In $\mathrm{Pa}$, the integral role of T3S in initiating $\mathrm{Pa}$ infections is supported by the finding that cellular susceptibility to $\mathrm{Pa}$ infections parallels cellular sensitivity to T3S $(33,206,271)$. Consistent with T3S being integral to $P a$ infections, this study used manipulations of the T3S effector ExoS to help dissect the cellular mechanism underlying the Pa infectious process.

$\mathrm{Pa}$ utilizes T3S to modulate host cell function through four identified effectors, ExoS, ExoT, ExoU, and ExoY. ExoS and ExoT are homologous, bifunctional proteins that include Rho GTPase activating protein (GAP) $(116,166)$ and ADP-ribosyltransferase (ADPRT) activities $(147,341)$. ExoS functions in $\mathrm{Pa}$ pathogenesis $(130,297,320)$ and is expressed by $58-72 \%$ of clinical isolates (129). The contribution of ExoT to disease appears limited $(183,290,320)$, but its expression by $92-100 \%$ of isolates (129) implies its importance to $\mathrm{Pa}$ survival. ExoU has phospholipase A2 activity and causes rapid loss of plasma membrane integrity and cell death (278). The mutually exclusive presence of exoS or exoU genes dictates whether $\mathrm{Pa}$ invades or 
lyses cells, respectively, and expression of ExoU is associated with more virulent $\mathrm{Pa}$ infections $(90,285,290)$. ExoY is an adenylate cyclase found in $70-95 \%$ of clinical isolates $(82,347)$ and may contribute to $\mathrm{Pa}$ systemic spread (320). The least understood stage in T3S is the translocation of effectors across host cell membranes. In $\mathrm{Pa}$ this requires three proteins, membrane channel forming PopB and PopD, and channel assembling PcrV (215).

Polarized epithelial monolayers and undifferentiated HL-60 promyelocytic cells have the common property of being resistant to $P a$ infections and T3S effector translocation $(206,271)$. Both cell culture models also have the potential to become sensitive to $P a$ infections and T3S: in epithelial monolayers this occurs in conjunction with disruption of apical-basolateral polarity, and in HL-60 cells this is induced by differentiation with phorbol esters $(206,271)$. Previous studies in our laboratory characterizing host cell properties that influence the establishment of $\mathrm{Pa}$ infections identified a relationship between host cell leading edge properties involved in cell migration and cellular sensitivity to T3S translocated ExoS (33). The epithelium is structured to resist pore formation as occurs during T3S translocation, and the observed relationship between leading edge properties and T3S translocation led us to hypothesize that actin-plasma membrane associations at the leading edge alter membrane properties to facilitate T3S and the establishment of $\mathrm{Pa}$ infections.

The small size of the T3S translocon pore currently precludes direct examination of translocon channel formation in relation to leading edge properties of migrating epithelial cells. As an alternative approach for assessing the role of cell migration in T3S translocation, this study used ExoS with mutations in its GAP or ADPRT activities to manipulate T3S, and then examined how these manipulations influenced the Pa infectious process in highly migratory T24 epithelial cells. Our studies found that Pa expressing wild type (WT) ExoS preferentially bound to the actin-rich leading edge region of T24 cells, where T3S translocated ExoS gained access to actin filaments that facilitated its internalization. T3S translocated ExoS ADPRT activity then interrupted T3S in 
conjunction with targeting cellular proteins that disrupt actin-plasma membrane association and leading edge architecture. No alterations in leading edge architecture were observed when mutations in both ExoS GAP and ADPRT activities were introduced, and this led to enhanced T3S translocation and $\mathrm{Pa}$ internalization, and $\mathrm{Pa}$ aligned with actin filaments as it migrated to the perinuclear region of T24 cells. Collectively, our studies found that ExoS plays a paradoxical role in $\mathrm{Pa}$ infections by contributing to $\mathrm{Pa}$ toxicity, but its toxic effects also interrupt T3S translocation and $\mathrm{Pa}$ internalization to limit the $\mathrm{Pa}$ infectious process. 


\section{MATERIALS AND METHODS}

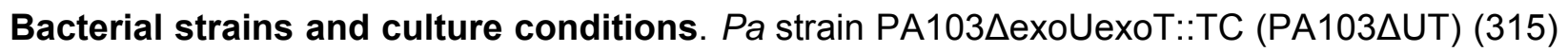
that lacks known T3S effectors, and strain PA103 $\triangle \mathrm{PopB}$, a T3S translocon mutant, were provided by Dara Frank (Medical College of Wisconsin, Milwaukee, WI). Wild type ExoS with a hemagglutinin tag (ExoS-HA) and $P a$ expressing plasmid encoded ExoS-R146K/E379D/E381D3XFLAG (ExoS-KDD-3XFLAG) were provided by Joseph Barbieri (Medical College of Wisconsin). PA103 UUT was used to express plasmid encoded ExoS enzymatic mutants, described in Table II. The ExoS-E379A-ADPRT- mutant and ExoS-R146A-GAP-/E379A ADPRT- mutant were constructed for this study using the Quick Change PCR-based site directed mutagenesis (Strategene, La Jolla CA), as previously described (20). The E379A mutation was introduced into the Pstl-BamHI fragment of the exoS gene cloned into the pUCP vector (65) using the following primers: forward, 5'GGATATCGAACTACAAGAATGCAAAAGAGATTCTCTATAAC-3' and reverse, 5'GTTATAGAGAATCTCTTTTGCATTCTTGTAGTTCGATATCC-3'. The R146A/E379A double mutant was constructed using the E379A primers and pUCP encoded ExoS-R146A mutant as template. All bacterial strains were grown in ExoS induction medium (TSBD-N) (147) for 14-16 hr prior to co-culture with eukaryotic cells.

Eukaryotic cell culture and bacterial-eukaryotic cell co-culture conditions. T24 cells were obtained from American Type Cell Culture Collection (ATCC, Manassas VA) and cultured in McCoy's 5A medium containing 10\% fetal bovine serum (FBS), $100 \mathrm{U} / \mathrm{ml}$ penicillin and 100 $\mu \mathrm{g} / \mathrm{ml}$ streptomycin (Hyclone, Logan UT). For Pa-T24 cell co-culture studies, T24 cells were seeded at $0.5-0.75 \times 10^{5}$ cells $/ \mathrm{ml}$, grown for 24 or $48 \mathrm{hr}$ and washed twice in medium containing $0.6 \%$ bovine serum albumin (BSA; Sigma-Aldrich, St. Louis $\mathrm{MO}$ ), prior to addition of $10^{7} \mathrm{CFU} / \mathrm{ml}$ Pa (MOI: 10-15 Pa per T24 cell). T24 cells were infected with $P a$ for $1.5-4.5 \mathrm{hr}$, as indicated, depending on the type of analysis. 
Analysis and quantification of T3S translocation. Cells were seeded for $48 \mathrm{hr}$ and cocultured with $\mathrm{Pa}$ for $3.5-4 \mathrm{hr}$, which is the time required to detect T3S translocation in T24 cells by immunoblot analysis. Pa were removed, cells were washed twice in medium containing 200 $\mu \mathrm{g} / \mathrm{ml}$ ciprofloxacin, and cells were detached and extracellular proteins degraded with $0.05 \%$ trypsin-1 mM EDTA (Hyclone). An aliquot of cells was assayed for viability based on trypan blue exclusion, and viable and total cell numbers were enumerated using a hemacytometer. A second aliquot of cells was examined for total protein using the BCA Protein Assay (Pierce, Rockford IL). Remaining cells were resuspended in digitonin fractionation buffer and fractionated as previously described (264).

Membrane and cytosolic fractions were assayed for ExoS and PopB translocation by SDSPAGE and immunoblot analysis based on equal protein loading. RalA served as a membrane fraction loading control, and ADP-ribosylation of RalA provided a functional assay of T3S translocated ExoS ADPRT activity (96). ExoS and PopB translocation into membrane fractions was quantified by densitometry using ImageJ $1.40 \mathrm{~g}$ software (http://rsbweb.nih.gov/ij/) and normalized with RalA density for each sample. Glyceraldehyde 3-phosphate dehydrogenase (GAPDH) served as a cytosolic fraction loading control, and ExoS translocation into cytosolic fractions was quantified and normalized with GAPDH density for each sample. Antibodies used in immunoblot analyses included: anti-HA (Covance Research, Princeton NJ), anti-PopB (provided by Joseph Barbieri), anti-RalA and anti-ezrin (BD-Transduction, San Jose CA), antiGAPDH (Millipore, Temecula CA), and anti-phospho-ERM (pERM, Cell Signaling, Danvers MA).

Analysis of T24 cell adherence and morphology. To assay effects of ExoS mutants on T24 cell adherence, cells were seeded $48 \mathrm{hr}$ prior to co-culture with $\mathrm{Pa}$ for $4 \mathrm{hr}$, a co-culture time that allowed detection of toxic effects of ExoS on T24 cell adherence to tissue culture wells. Cells were washed, detached, and stained with trypan blue as in immunoblot analyses, and 
adherent cells were enumerated using a hemacytometer. Percent T24 cell adherence to tissue culture wells was calculated relative to total cells in uninfected control wells.

T24 cell rounding was visually estimated following a 3, 3.5, 3.75, and $4 \mathrm{hr}$ co-culture period. Differences in the efficiency of cell rounding were most evident following a 3-3.5 hr co-culture period, which are reported in Table 1 . Cell rounding progressed to $85-95 \%$ following a $4 \mathrm{hr}$ coculture period with $\mathrm{Pa}$ expressing ExoS with active GAP and / or ADPRT activities.

Immunofluorescence analysis. T24 cells were seeded in chamber slides (Nalge Nunc International, Rochester NY) $24 \mathrm{hr}$ prior to co-culture with $\mathrm{Pa}$ for the indicated times, then washed twice in Dulbecco's Phosphate-Buffered Saline (DPBS; Hyclone). The site of Pa binding to T24 cells was determined after a $1.5 \mathrm{hr}$ co-culture period and examined by fixing cells in $3 \%$ paraformaldehyde in actin stabilization buffer $(A S B)(33,115)$, permeabilizing with $0.2 \%$ Triton$\mathrm{X} 100$ in $\mathrm{ABS}$, and blocking with $0.8 \% \mathrm{BSA}, 0.1 \%$ fish gelatin, and $10 \mu \mathrm{g} / \mathrm{ml}$ goat $\mathrm{lgG}$. Cells were stained for: F-actin using TRITC-phalloidin (Sigma-Aldrich), Pa using anti-Pa LPS (Joseph Lam, University of Guelph, Guelph, Ontario); and trailing edge using anti-caveolin-1 (N-20; Santa Cruz Biotechnology, Santa Cruz CA). Pa and caveolin-1 were visualized using an anti-rabbit Alexa Fluor 647 conjugate (Invitrogen, Carlsbad CA). Bacterial localization to the leading edge, lamellipodium, perinuclear, and trailing edge of T24 cells was visually enumerated.

To assay ExoS translocation and $\mathrm{Pa}$ internalization, cells were co-cultured with $\mathrm{Pa}$ for 1.25 to 3 $\mathrm{hr}$ as indicated, fixed, and blocked as above. External Pa were stained using anti-Pa LPS, followed by blocking with 6\% BSA in DPBS and visualized using a Qdot 655 goat anti-rabbit conjugate (Invitrogen). Cells were fixed again, permeabilized, blocked, and all Pa were stained with anti-Pa LPS followed by an Alexa Fluor 647 conjugate (Invitrogen). Internal Pa were differentiated by absence of Qdot 655 staining. ExoS was detected using anti-HA and an antimouse Alexa Fluor 488 conjugate. Detection of $\mathrm{pERM}$ following infection with $\mathrm{Pa}$ strains 
required fixation with $10 \%$ trichloroacetic acid on ice for $10 \mathrm{~min}$. Cells were then stained for external $\mathrm{Pa}$ and total $\mathrm{Pa}$, as previously described, and pERM and total ezrin were detected using specific antibodies, indicated above. Total $\mathrm{Pa}$ and $\mathrm{pERM}$ were visualized using an antirabbit Alexa Fluor 647 conjugate.

Following staining procedures cells were mounted using Fluoromount-G (Southern Biotechnology Associates, Birmingham $\mathrm{AL}$ ) and examined using a Plan-Apochromat 63x/1.40 oil objective on a Zeiss Imager.Z1 LSM 510 confocal microscope (Jena, Germany). Images were exported to Adobe Photoshop CS4 Extended (Adobe Systems Incorporated, San Jose, CA) as 8 bit TIFF files, where immunofluorescence intensity levels were adjusted equivalently.

Co-immunoprecipitation of actin with ExoS. To examine actin association with T3S translocated ExoS, T24 cells were seeded in $100 \mathrm{~mm}$ dishes, grown to $90 \%$ confluency and cocultured for $4.5 \mathrm{hr}$ with PA103AUT expressing ExoS-KDD-3XFLAG. Cells were washed twice with DPBS and detached with $0.25 \%$ trypsin (Hyclone). Trypsin was neutralized with FBS, and cells were pelleted by low-speed centrifugation and resuspended in HB2 buffer (3 mM imidazole, pH 7.4, 250 mM sucrose, containing PIC protease inhibitor cocktail; Sigma) (352), and kept on ice. Cells were lysed by passage through a 27 gauge needle, unbroken cells and nuclei were pelleted by centrifugation $(16,000 \mathrm{~g}$ for $5 \mathrm{~min}$ ), and proteins were precipitated with anti-FLAG M2-Agarose affinity resin (Sigma), as previously described (352). Proteins were eluted with $300 \mathrm{ng}$ of 3XFLAG peptide, resolved by SDS-PAGE, and silver stained.

To identify ExoS interacting proteins, bands from two gels were excised, and silver ions were removed by adding fresh destaining solution (15 $\mathrm{mM}$ potassium ferricyanide, $50 \mathrm{mM}$ thiosulfate in distilled water) (Invitrogen). Samples were rinsed twice with distilled water, equilibrated with $100 \mathrm{mM}$ ammonium bicarbonate (Fisher Scientific, Pittsburgh PA), rinsed three times in 50\% acetonitrile (ACN, Fisher Scientific) in $100 \mathrm{mM}$ ammonium bicarbonate $\left(23^{\circ} \mathrm{C}, 15 \mathrm{~min}\right)$, and 
dehydrated in $100 \%$ ACN. Dehydrated gel samples were trypsinized in situ with $200-300 \mathrm{ng}$ trypsin (Proteomics Grade, Sigma) for $14 \mathrm{hr}$, and peptides were extracted twice with 50\% ACN, $2.5 \%$ trifluoroacetic acid (Fisher Scientific) in distilled water and dried using a CentriVap Speed Vacuum. Peptides were then mixed with $5 \mu$ lpha-Cyano-4-hydroxycinnamic acid (Applied Biosystems, Carlsbad CA), and $0.5 \mu \mathrm{l}$ was spotted onto the target for MALDI-TOF analysis. MALDI-TOF was performed using the 4700 Proteomics Analyzer (Applied Biosystems), which was calibrated using CalMix (Applied Biosystems) with the following instrument settings: (i) minimum signal to noise of 10 , (ii) mass tolerance of $\pm 2 \mathrm{~m} / \mathrm{z}$, (iii) minimum peaks to a match of 6, (iv) maximum outlier error of $5 \mathrm{ppm}$, and (v) a laser intensity of 3900. Mass spectra were individually calibrated using internal trypsin peaks with Data Explorer software (Applied Biosystems). Proteins were identified using ProteinProspector (http://prospector.ucsf.edu/ prospector/mshome.htm), set to a mass accuracy of $\pm 150 \mathrm{ppm}$ to compare unknown mass fingerprints to those of known proteins in the $\mathrm{NCBI}$ database using a species-specific filter for Homo sapiens or Pseudomonas aeruginosa.

Statistical analysis. JMP Version 9 Software (SAS Institute, Cary, NC) was used for statistical analyses, and significance was determined by a two-sided alpha level set at $P<0.05$. A one-way ANOVA was used to evaluate differences among $\mathrm{Pa}$ strains relative to ExoS/RalA and PopB/RalA concentrations in membrane fractions and the site of $\mathrm{Pa}$ localization on T24 cells. The number of internal or external Pa per T24 cell was evaluated by a least squared means. Relative intensity of pERM was analyzed by a randomized complete block. For all data, multiple comparisons were analyzed with a Tukey's HSD test. Pearson's correlation coefficient was used to assess associations between continuous variables. 
Table II. ExoS mutant activity and cellular toxicity

\begin{tabular}{|c|cc|ccc|c|}
\hline & Enzyme activity & \multicolumn{3}{|c|}{ Effect on T24 cells $^{*} \begin{array}{c}\text { Source } \\
\text { or } \\
\text { Plasmid construct }\end{array}$} \\
\cline { 2 - 6 } & GAP & ADPRT & $\begin{array}{c}\% \\
\text { Rounding }\end{array}$ & $\begin{array}{c}\% \\
\text { Viability }^{3}\end{array}$ & $\begin{array}{c}\% \\
\text { Adherence }^{4}\end{array}$ & Reference \\
R146A-HA & - & + & 32.5 & 51.2 & 32.5 & $(95)$ \\
WT ExoS-HA & + & + & 22.5 & 51.3 & 28.4 & $(171)$ \\
E381A-HA & + & Reduced & 35 & 96.9 & 48.5 & $(188)$ \\
E379A/E381A-HA & + & - & 34 & 97.7 & 78.0 & $(95)$ \\
R146A/E381A-HA & - & Reduced & 0 & 80.9 & 62.7 & $(95)$ \\
E379A-HA & + & - & 11.3 & 98.6 & 68.1 & This study \\
R146A/E379A-HA & - & - & 0 & 87.2 & 80.6 & This study \\
R146A/E379A/E381A-HA & - & - & 0 & 88.6 & 80.3 & $(95)$ \\
pUCP & - & - & 0 & 93.1 & ND & $(287)$ \\
\hline
\end{tabular}

${ }^{1}$ ExoS constructs are listed from highest to lowest cytotoxicity, ranked based on additive toxic effects of the construct on T24 cell rounding, viability and adherence.

${ }^{2}$ Mean T24 cell rounding, determined after a 3-3.5 hr co-culture period.

${ }^{3}$ Mean T24 cell viability, determined based on trypan blue exclusion analyses after a 3.5-4 hr co-culture period.

${ }^{4}$ Mean percent T24 cells adherent to tissue culture wells after a $4 \mathrm{hr}$ co-culture period.

${ }^{5}$ Not determined. 


\section{RESULTS}

Characterizing the influence of ExoS enzyme activity on T3S translocation in T24 cells. ExoS GAP and ADPRT activities have been found to alter ExoS translocation into eukaryotic cells, but the alteration varies with the cell culture system, and the mechanism underlying the alteration is unclear $(4,45,95)$. To evaluate how ExoS GAP and ADPRT activities influence T3S in T24 cells, cells were co-cultured for 3.5-4 hr with strain PA103AUT (which lacks T3S effectors but retains an intact T3S system (314)), expressing plasmid-encoded WT ExoS or ExoS with mutations in GAP (R146A), ADPRT (E379A, E381A, or E379A/E381A), or GAP/ADPRT (R146A/E379A, R146A/E381A, or R146A/E379A/E381A) activities (Fig. 15A). Cellular toxicity of $\mathrm{Pa}$ ExoS mutants, ranked based on T24 cell rounding, viability, and adherence to tissue culture wells is shown in Table 1, and ExoS-R146A was identified as the most cytotoxic and ExoS-R146A/E379A/E381A as the least cytotoxic form of ExoS. ExoS translocation into membrane fractions was quantified based on immunoblot analysis, and a progressive increase in translocation was observed with WT < GAP- < ADPRT- < GAPIADPRT- mutants (Fig. 15B). Quantification of ExoS translocation confirmed significant differences between group A (WT and GAP- mutant) and group C (GAP-/ADPRT- mutants) $(P<0.001)$, and between group B (ADPRT- mutants) and group C (GAP-/ADPRT- mutants) $(P<0.05)$ (Fig. 15C). Translocation of WT ExoS was also significantly less $(P<0.05)$ than that of ExoS ADPRT- mutants. Differences in ExoS translocation observed by ExoS GAP- or ADPRTmutants provided evidence that the two activities exerted different influences on T3S.

PopB membrane insertion was used as a measure of general T3S translocation and differed slightly from ExoS translocation in that mutations in ExoS ADPRT activity were less effective in enhancing PopB translocation. However, overall comparisons of PopB and ExoS membrane translocation identified a positive correlation $(r=0.829, P<0.001)$ between the two. A significant increase $(P<0.05)$ in PopB translocation was also observed by $P$ a expressing an ExoS 
R146A/E379A/E381A GAP-/ADPRT- mutant as compared to Pa expressing WT ExoS, ExoS GAP-, or ExoS ADPRT- mutants, indicating that ExoS GAP and ADPRT activities caused a general decrease in T3S translocation (Fig. 15C).

RalA ADP-ribosylation, recognized by a shift in mobility by SDS-PAGE analysis, provides a functional read-out of T3S translocated ExoS ADPRT activity (96), and Pa expressing ExoS with E381A mutations, but retaining an active E379 catalytic residue, showed a low level of ADPRT activity, as recognized by a slight shift in RalA mobility (Fig. 15B). This activity coincided with increased cellular toxicity, as shown by decreased adherence and/or increased cell rounding by E381A and R146A/E381A mutants, as compared to respective E379A and R146A/E379A mutants (Table II). Alterations in T3S translocation did not appear to relate to a negative feedback effect on ExoS expression by infected T24 cells, as determined by the relatively equal concentrations of ExoS mutants and PopB in co-culture supernatants (Fig. 15D). The ability of mutations in ExoS GAP and ADPRT activities to enhance ExoS and PopB translocation indicated that ExoS was targeting cellular processes that affect T3S translocation, which if identified would provide insight into T24 cell properties that influence the Pa infectious process. 
Figure 15. Influence of ExoS activity on Pa-T3S translocation. A) Linear model of ExoS GAP and ADPRT domains showing key functional residues (20). B) T24 cells were co-cultured for 3.5-4 hr with strain PA103AUT expressing plasmid encoded WT ExoS or ExoS with mutations in GAP (R146A), ADPRT (E379A, E381A, E379A/E381A), or GAP/ADPRT (R146A/E379A, R146A/E381A, R146A/E379A/E381A) activities. pUCP is a vector control, and $\triangle \mathrm{PopB}$ is a translocon mutant control. Cells were harvested, fractionated and T3S translocation of ExoS and PopB into membrane fractions was assayed by immunoblot analysis. RalA modification is a functional read-out of translocated ExoS ADPRT activity, and asterisks mark ADP-ribosylated RalA. Total RalA served as a membrane fraction loading control. C) T3S translocated ExoS and PopB were quantified by densitometry and normalized with RalA density for each sample. Results are expressed as the mean ExoS/RalA or PopB/RalA ratio \pm SEM. Asterisks mark significant differences in ExoS translocation $\left({ }^{* *} P<0.001, A\right.$ vs. C; or ${ }^{*} P<0.05, B$ vs. C). PopB translocation by groups $A$ and $B$ are also significantly different $(P<0.05)$ from that of $\mathrm{Pa}$ expressing an ExoS-R146A/E379A/E381A GAP-/ADPRT- mutant (not indicated on graph). D) Co-culture supernatants were harvested and compared for ExoS and PopB expression by $\mathrm{Pa}$ in the presence of T24 cells based on immunoblot analysis. Results are representative of four independent experiments. 
A

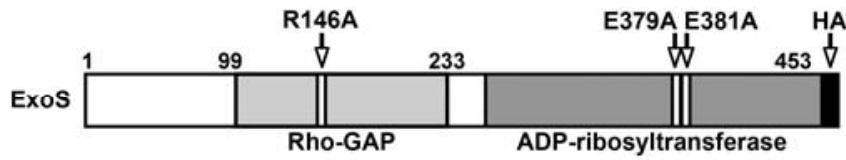

B

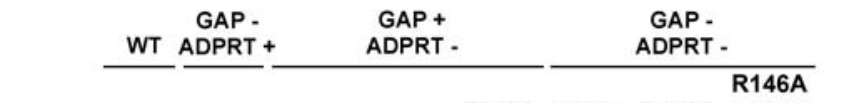

E379A R146A R146A E379A

- ExoS R146A E379A E381A E381A E379A E381A E381A pUCP $\triangle P$ PopB Pa strain
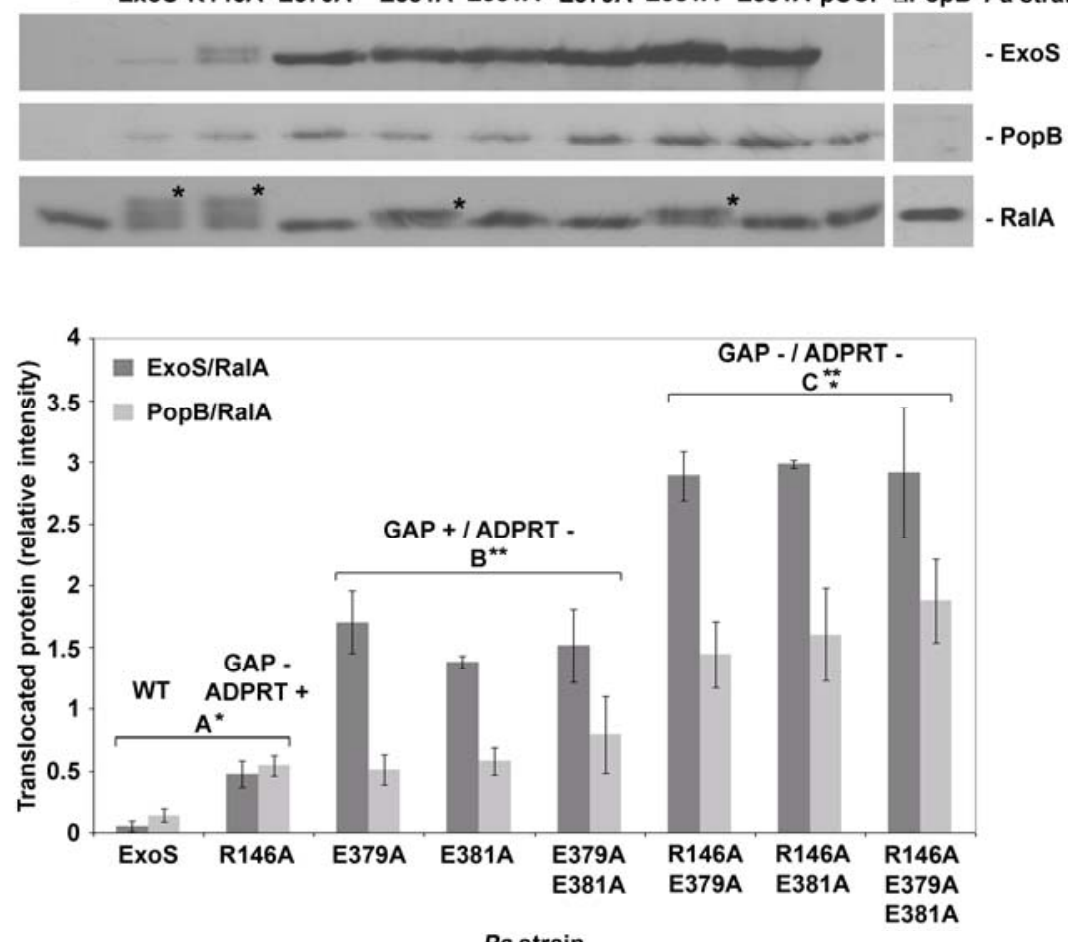

Pa strain

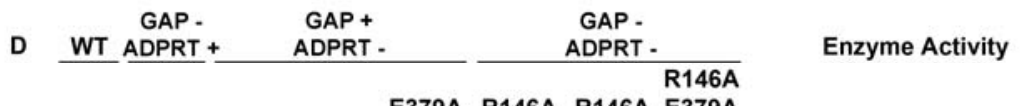

E379A R146A R146A E379A

ExoS R146A E379A E381A E381A E379A E381A E381A pUCP Pastrain

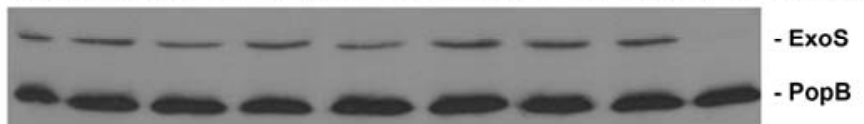


Examining $\mathrm{Pa}$ binding to T24 cells in relation to T3S translocation. T3S translocation requires $\mathrm{Pa}$ binding to eukaryotic cells, and another means of evaluating the role of leading edge properties and cell migration in Pa-T3S is to relate the site of $\mathrm{Pa}$ binding to T24 cells to ExoS translocation efficiency. T24 cells exhibit a highly migratory mesenchymal pattern characterized by an expansive lamellipodium with dense actin staining and membrane ruffling, and caveolin-1 staining that localizes to the trailing edge can be used to further define the direction of migration polarity (149) (Fig. 16A). Pa binding to T24 cells was examined using immunofluorescence staining and confocal microscopy (IF). After a $1.5 \mathrm{hr}$ co-culture, which allows $\mathrm{Pa}$ binding but causes minimal effects on cell morphology, $\mathrm{Pa}$ expressing WT ExoS and having the least efficient T3S translocation bound preferentially to the leading edge (Fig. 16B). In comparison, $\mathrm{Pa}$ expressing GAP-, ADPRT-, or GAP-/ADPRT- mutants and having more efficient T3S translocation bound primarily within the lamellipodium (Figs. 16C-E), as did the Pa pUCP vector control (Fig. 16F) and $\mathrm{Pa} \triangle \mathrm{PopB}$ mutant (not shown). Statistical analysis confirmed significantly more $(P<0.05) P a$ expressing WT ExoS bound to the leading edge of T24 cells than $\mathrm{Pa}$ pUCP or $\mathrm{Pa} \triangle \mathrm{PopB}$, indicating that ExoS GAP and ADPRT activities increased $\mathrm{Pa}$ binding to the leading edge.

To summarize, expression of WT ExoS correlated with Pa binding to the leading edge of T24 cells, but more efficient T3S translocation by ExoS ADPRT- or GAP-/ADPRT- mutants was associated with $\mathrm{Pa}$ binding within the lamellipodium. This finding was contradictory to our hypothesis that actin-plasma membrane associations at the leading edge facilitated T3S translocation, making it important to understand the mechanism underlying the increased T3S translocation by $\mathrm{Pa}$ binding within the lamellipodium. 
Figure 16. Site of binding of $\mathrm{Pa}$ expressing ExoS constructs to T24 cells. A) Diagram of T24 cell regions in relation to mesenchymal migration. Leading edge architecture, indicated by dense actin staining (white) and membrane ruffling was differentiated from the trailing edge by caveolin-1 staining (light blue). B-F) T24 cells were co-cultured for $1.5 \mathrm{hr}$ with Pa expressing the indicated ExoS construct. Left panels show representative images of the Pa binding site to T24 cells by $\mathrm{Pa}$ expressing the indicated ExoS construct. $\mathrm{Pa}$ (blue) binding is indicated by yellow arrows. Scale bar is $10 \mu \mathrm{m}$. Right panels show quantification of the percent of the respective $\mathrm{Pa}$ strain binding to the leading edge, lamellipodium, perinuclear, or trailing edge. $\mathrm{Pa}$ binding was enumerated based on visualizing the binding of each strain to an average of 74 T24 cells. The graph shows $\mathrm{Pa}$ binding to the indicated regions of the cell. Asterisks denote a significant increase $(P<0.05)$ in binding of $P a$ expressing WT ExoS to the leading edge as compared to $P a$ pUCP. 
Uninfected control

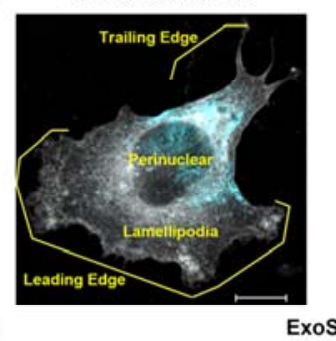

B Exos
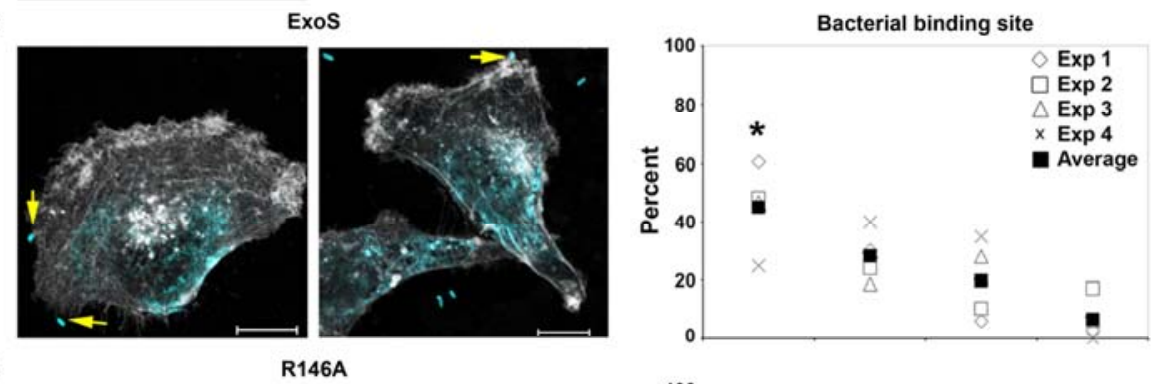

C
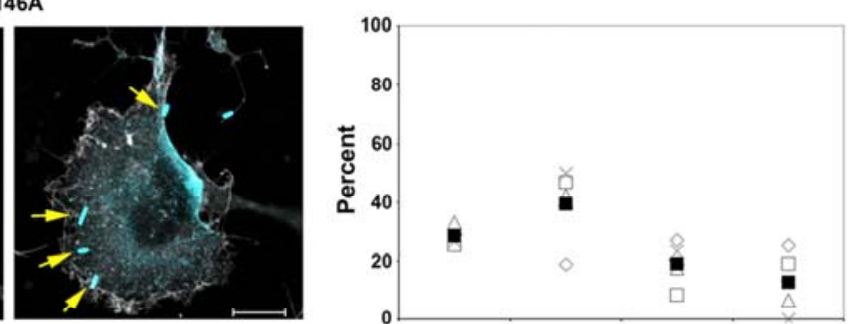

D

E379A / E381A
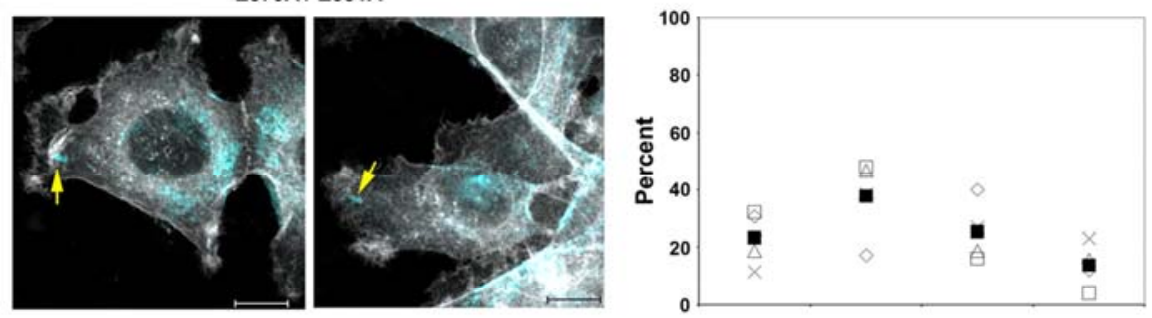

E

R146A/E379A/E381A
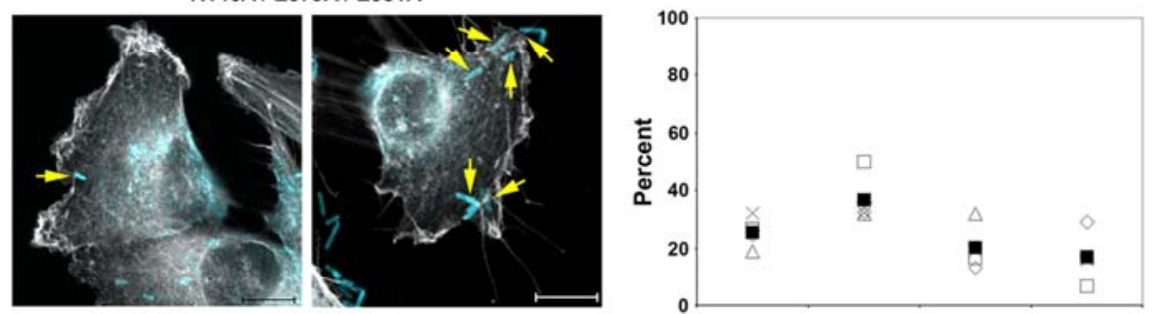

F

pUCP vector control
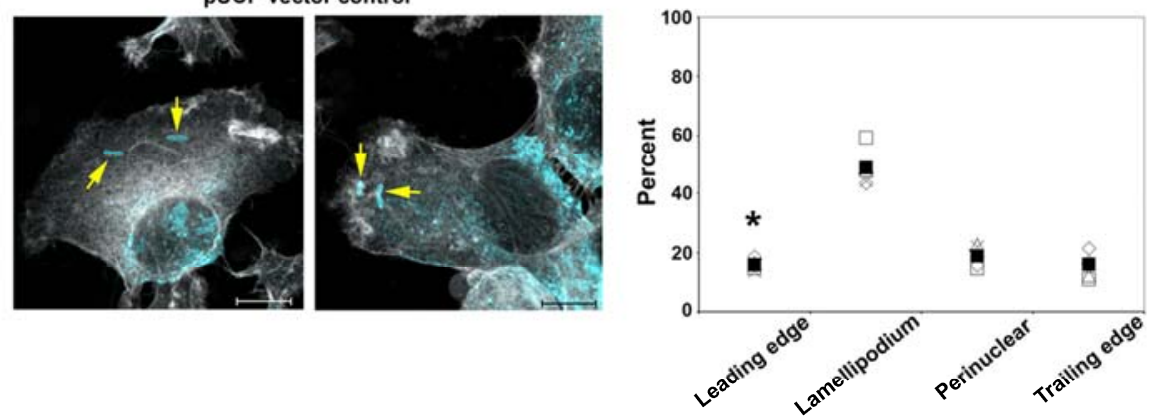
Examining T3S translocation by $\mathrm{Pa}$ expressing ExoS enzymatic mutants. To further explore the relationship between $\mathrm{Pa}$ binding to the leading edge or lamellipodium of T24 cells and the efficiency of T3S translocation, HA-tagged ExoS mutant constructs were used to monitor T3S translocation by IF following co-culture with $\mathrm{Pa}$ for 2.2 to $3 \mathrm{hr}$. T24 cells are less adherent to glass slides than to tissue culture wells, and a 2.2-3 hr co-culture period allowed assessment of $\mathrm{Pa}$ binding in relation to ExoS translocation, while the majority of T24 cells remained adherent. As ExoS GAP activity is known to have an anti-internalization function (52, $64,100,263)$, to differentiate ExoS translocation by external or internal $\mathrm{Pa}$, a double $\mathrm{Pa}$ IF staining protocol was developed, staining extracellular Pa with Qdot 655 and total Pa with Alexa Fluor 647, while also staining for ExoS using an anti-HA antibody. Visualization of external $\mathrm{Pa}$, internal $\mathrm{Pa}$, and ExoS using this protocol is shown in Supplementary Figure S6.

As previously indicated (Fig. 16B), when T24 cells were co-cultured with Pa expressing WT ExoS for $1.5 \mathrm{hr}$, the leading edge remained intact and extracellular $\mathrm{Pa}$ bound primarily to the leading edge. Increasing the co-culture time with WT ExoS beyond $1.5 \mathrm{hr}$ led to a rapid decline in leading edge architecture, as recognized by the loss of dense actin staining in the direction of cell migration (Fig. 17B) in comparison to cells co-cultured with the Pa pUCP vector control (Fig. 17A). Consistent with immunoblot analyses, translocation of WT ExoS into T24 cells was difficult to detect by IF but was apparent based on T24 cell rounding and loss of migration polarity. WT ExoS also disrupted actin linkage to the plasma membrane as evident in the loose wavy actin filaments extending from T24 cells (Fig. 17B, indicated by blue arrows). Preferential binding of $\mathrm{Pa}$ expressing WT ExoS to the leading edge could in turn be related to the leading edge being an early site of $\mathrm{Pa}$ contact, but upon ExoS translocation and loss of cell architecture, $\mathrm{Pa}$ binding to other regions of T24 cells became less distinguishable.

As with WT ExoS, co-culture of T24 cells with Pa expressing an ExoS-R146A GAP- mutant led to loss of actin linkage to the plasma membrane (Fig. 17C, blue arrows), which was attributed to 
ExoS ADPRT activity shared by both WT ExoS and the ExoS GAP- mutant. As anticipated, Pa internalization was detected when ExoS GAP was mutated, and ExoS staining was found to colocalize with internalized $\mathrm{Pa}$ (Fig. 17C, marked by yellow arrow in panel 1). This finding indicated that ExoS was being expressed by internalized $\mathrm{Pa}$ and identified an alternative mechanism of translocating ExoS into T24 cells. This mode of translocation will be referred to as T3S translocation by internalized $\mathrm{Pa}$ in our studies to distinguish it from conventional T3S translocation by external $\mathrm{Pa}$. The binding of a $\mathrm{Pa}$ ExoS GAP- mutant within the lamellipodium of T24 cells, as shown in Figure $16 \mathrm{C}$, can now also be related to internalized $\mathrm{Pa}$ localizing within the lamellipodium.

IF studies revealed further increases in T3S translocation by $\mathrm{Pa}$ expressing an ExoSE379A/E381A ADPRT- mutant, as compared to WT ExoS and an ExoS GAP- mutant. Pa ExoS ADPRT- mutants remained extracellular and bound predominantly within the lamellipodium, and both diffuse and punctate ExoS staining patterns were observed (Fig. 17D). Punctate ExoS staining in close association with extracellular $\mathrm{Pa}$ may reflect sites of T3S membrane translocation (Fig. 17D, panel 2), and if so, this was detected by $P a$ within the lamellipodium, perinuclear, and leading edge regions of T24 cells. ExoS ADPRT- mutants maintain GAP activity, which altered T24 cell morphology causing cellular projections that had high intensity ExoS staining. Our interpretation of these results was that $P a$ expressing an ExoS ADPRTmutant translocated ExoS at sites other than the leading edge, but that increased ExoS staining in projections that extended from the leading edge related to this being an early site of T3S translocation.

Even higher levels of T3S translocation were evident in IF studies by $\mathrm{Pa}$ expressing an ExoSR146A/E379A/E381A GAP-/ADPRT- mutant, and this related to T3S translocation by internalized $\mathrm{Pa}$ (Fig. 17E). ExoS GAP-/ADPRT- staining was initially detected in close association with internalized $\mathrm{Pa}$ (Fig. 17E, panel 1), and then became more diffuse as $\mathrm{Pa}$ 
migrated to the perinuclear region of T24 cells (Fig. 17E, panels 2 and 3). Diffuse ExoS staining is interpreted to relate to the translocation and diffusion of ExoS within the cytosol by internalized $\mathrm{Pa}$. Intense ExoS staining co-localizing with $\mathrm{Pa}$ is interpreted to reflect localized induction or secretion of ExoS. Intense ExoS staining surrounding $\mathrm{Pa}$ is presumed to relate to ExoS secretion by $\mathrm{Pa}$ contained within a membrane vesicle (Fig. 17E, particularly evident in panel 3). If these interpretations are correct, ExoS induction can occur when Pa are localized within the lamellipodium and perinuclear regions of T24 cells, indicating that ExoS is being differentially induced as $\mathrm{Pa}$ migrate to the perinuclear region of T24 cells. Consistent with this possibility, time course studies detected a progression in ExoS GAP-/ADPRT- mutant staining (shown in Fig. 17F): (1) at early co-culture times (1 hr $15 \mathrm{~min}$ ) ExoS was detected in association with extracellular Pa; (2) with increasing time (1 hr $45 \mathrm{~min}$ ) Pa entered T24 cells and ExoS was detected in close association with internalized $\mathrm{Pa}$; (3) by $2 \mathrm{hr} 15 \mathrm{~min}$, ExoS was detected in close association with $\mathrm{Pa}$ migrating to the perinuclear region; and (4) by $3 \mathrm{hr}$, dense ExoS staining was localized to the perinuclear region of T24 cells where a large number of $\mathrm{Pa}$ resided. Subsequent T24 cell lysis and plating studies found that internalized Pa ExoS GAPIADPRT- mutants remained viable within T24 cells in numbers consistent with that observed in IF studies (data not shown).

To summarize, in examining the role of ExoS GAP and ADPRT activity in T3S translocation in T24 cells it was found that: (1) WT ExoS facilitates Pa binding to the leading edge, but ExoS GAP and ADPRT activity rapidly disrupt leading edge properties to inhibit T3S translocation by external $\mathrm{Pa}$; (2) mutations in ExoS GAP activity enhance T3S translocation, and this relates $\mathrm{Pa}$ internalization and translocation of ExoS within cells; (3) mutations in ExoS ADPRT activity enhance T3S translocation by external $\mathrm{Pa}$, and this occurs in conjunction with T3S translocation remaining engaged as $\mathrm{Pa}$ move from the cell periphery to within the lamellipodium; and (4) mutations in both ExoS GAP and ADPRT activity further enhance T3S translocation, and this 
relates to $\mathrm{Pa}$ internalization and continued T3S translocation as $\mathrm{Pa}$ migrate to the perinuclear region of T24 cells. 
Figure 17. Visualizing the effects of ExoS enzymatic activity on T3S translocation in relation to $\mathbf{P a}$ internalization. A-E) T24 cells were co-cultured for $2.2-3 \mathrm{hr}$ with $\mathrm{Pa}$ expressing a: A) pUCP vector control, or HA-tagged B) WT ExoS C) ExoS GAP-, D) ExoS ADPRT-, or E) ExoS GAP-/ADPRT- mutant. ExoS was visualized relative to $P a$ internalization and alterations in cell morphology. Cells were stained for external Pa (yellow + pink), internal Pa (pink), ExoS (green), and actin (white). Yellow arrows indicate internalized Pa. Blue arrows indicate loose actin. White arrows indicate direction of migration polarity. Images are representative of observations from six independent studies. Scale bar is $10 \mu \mathrm{m}$. F) A time course study was performed examining ExoS and $\mathrm{Pa}$ internalization following co-culture of T24 cells with $\mathrm{Pa}$ expressing an HA-tagged ExoS GAP-/ADPRT- mutant for $1 \mathrm{hr} 15 \mathrm{~min}, 1 \mathrm{hr} 45 \mathrm{~min}, 2 \mathrm{hr} 15 \mathrm{~min}$, or $3 \mathrm{hr}$. Cells were stained as above and examined for ExoS and $\mathrm{Pa}$ internalization relative to increasing co-culture time. Bottom panels show an enlarged view of the respective upper image. Red arrows in the $1 \mathrm{hr} 15$ min images mark ExoS GAP-/ADPRT- mutant staining by external $P a$. Scale bar is $10 \mu \mathrm{M}$. 
A

B

pUCP

Control
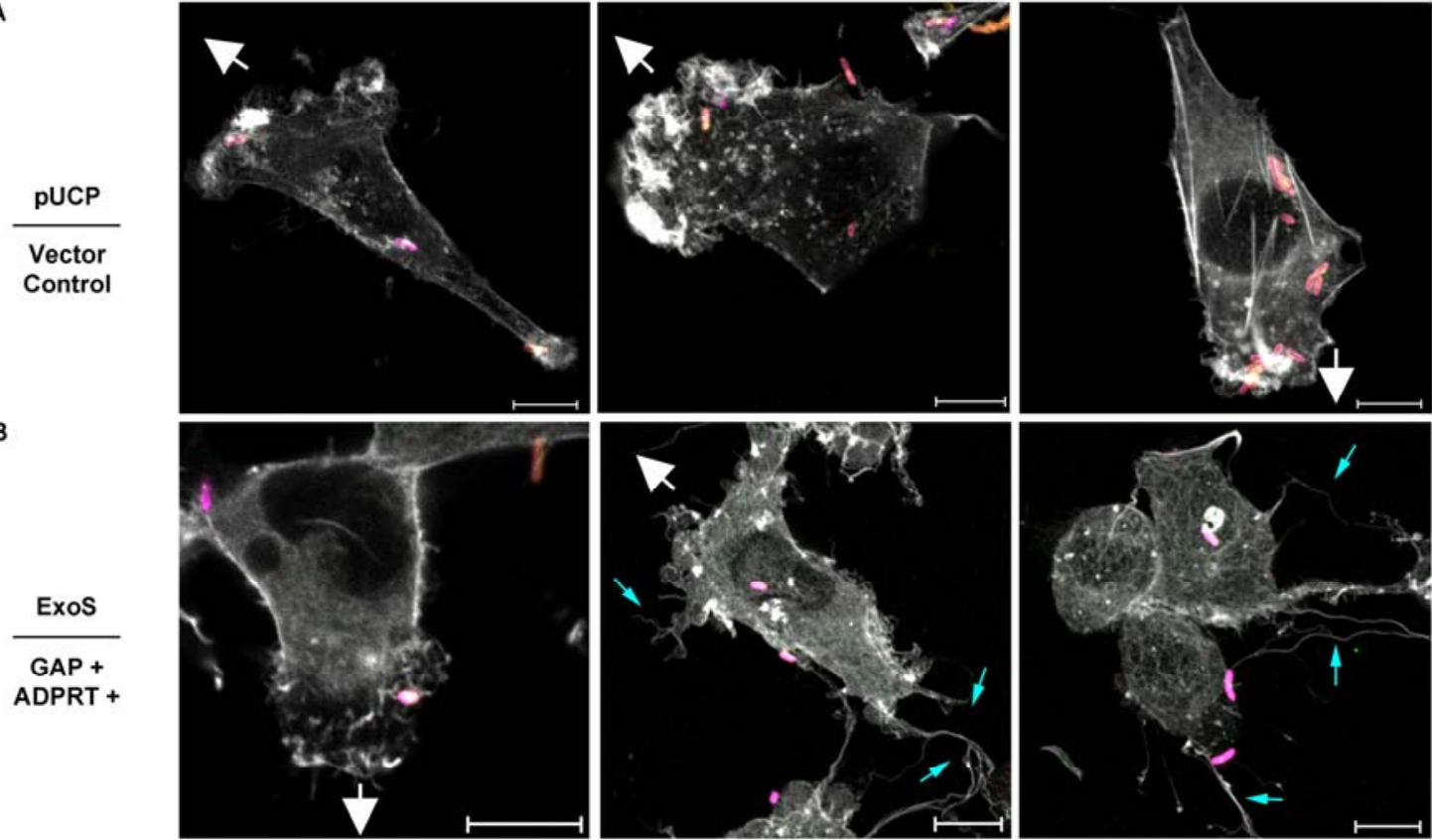

C
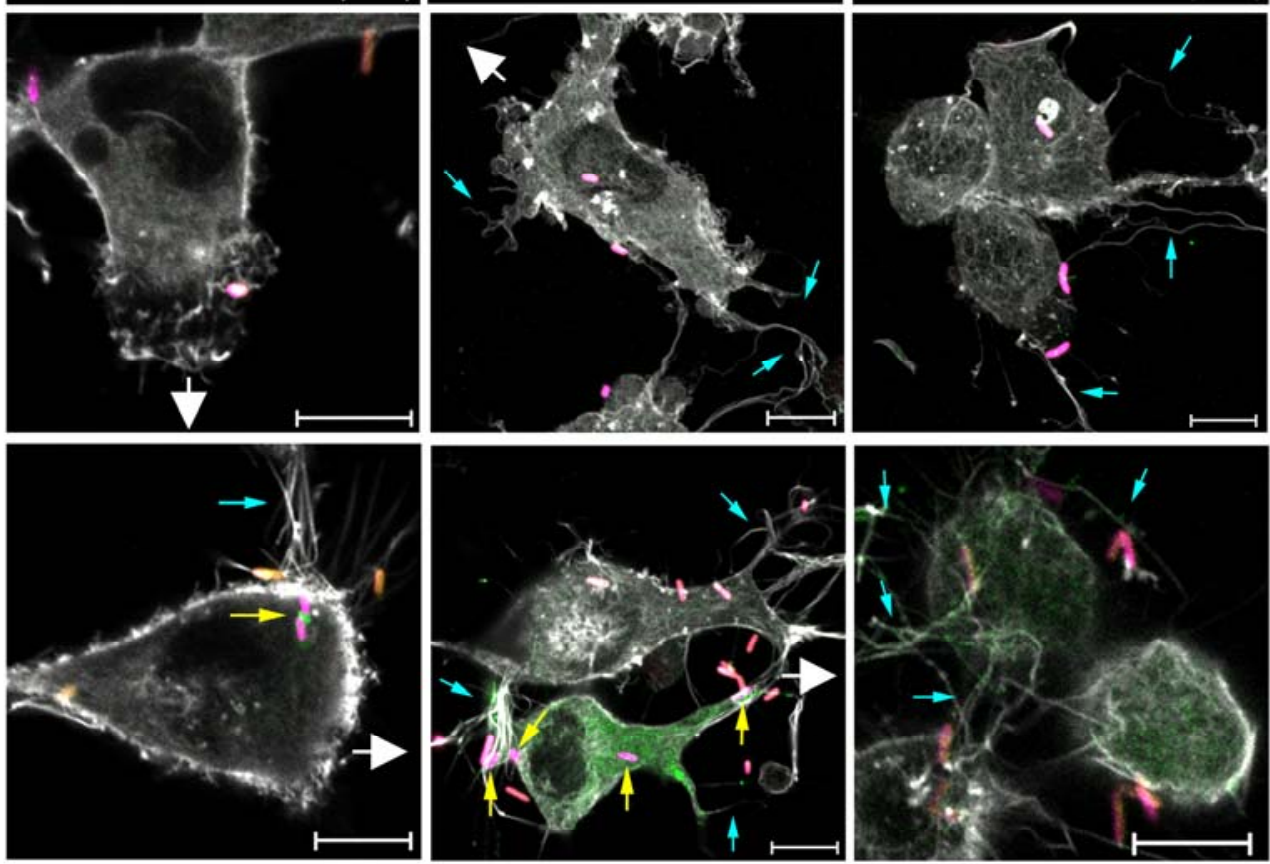

D
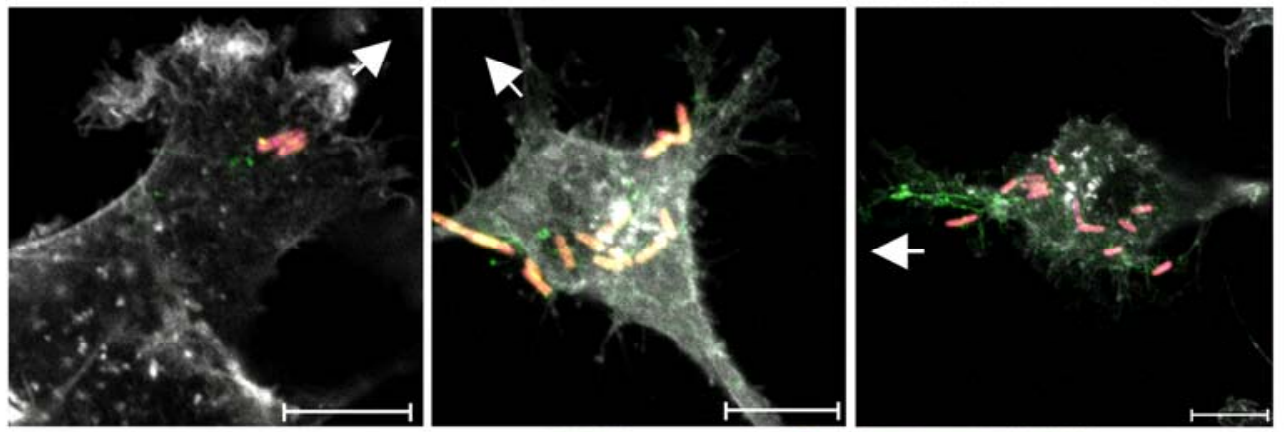

E
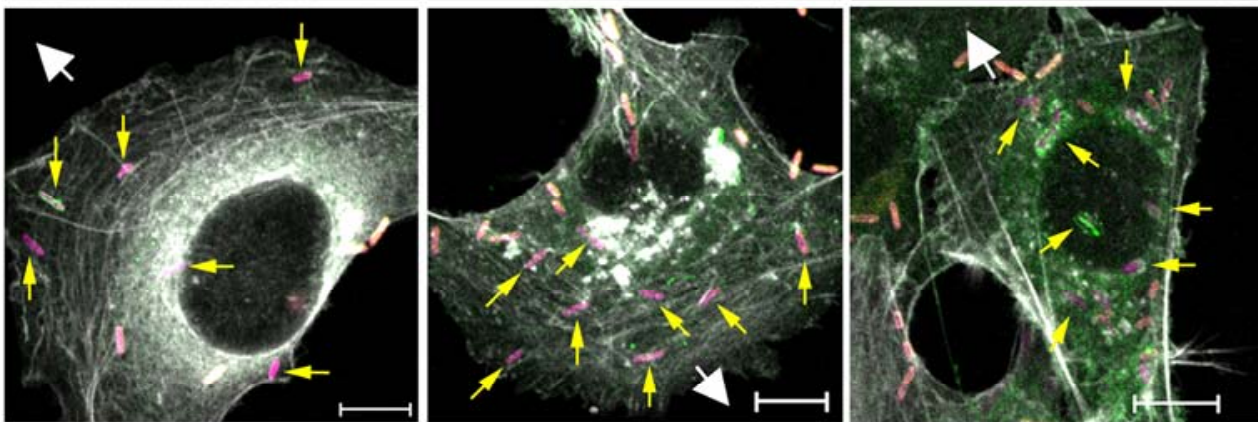


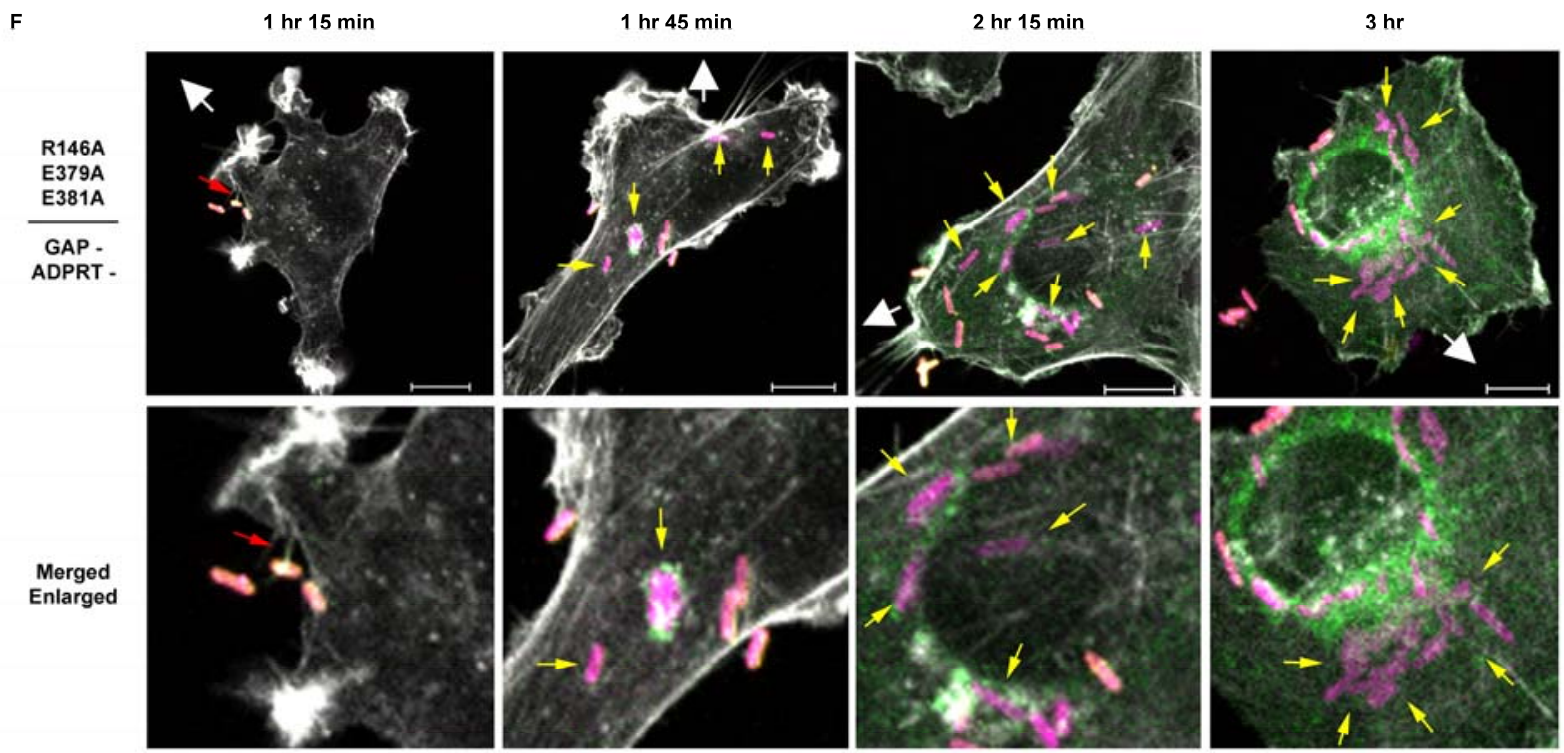


Examining T3S translocation by internalized $\mathrm{Pa}$. We attributed enhanced T3S translocation by a $\mathrm{Pa}$ ExoS GAP- mutant to ExoS translocation by internalized $\mathrm{Pa}$, but the mechanism underlying the greater levels of translocation of an ExoS GAP-/ADPRT- mutant remained unclear. When $\mathrm{Pa}$ internalization by ExoS mutant strains was quantified, Pa ExoS GAPIADPRT- mutants (R146A/E379A, R146A/E381A, or R146A/E379A/E381A) were found to be internalized at a significantly higher frequency $(P<0.001)$ than the other $P a$ strains, including the $P a$ ExoS-R146A GAP- mutant (Fig. 18A). In comparing $P a$ internalization to $P a$ binding to the external surface of T24 cells, a significant decrease $(P<0.05)$ in $P a$ binding was detected by $P a$ expressing WT ExoS and the ExoS GAP- mutant, as compared the other ExoS mutant strains (Fig. 18A). These results demonstrated that ExoS ADPRT activity affected T3S translocation in two ways: 1) its presence induced toxic effects on T24 cells that decreased $\mathrm{Pa}$ binding; and 2) its absence in combination with a GAP- mutation increased $P a$ internalization.

Increased $\mathrm{Pa}$ internalization by $\mathrm{Pa}$ expressing ExoS GAP-/ADPRT- mutants was also reflected in higher levels of cytosolic ExoS, as determined in cell fractionation and immunoblot analyses (Fig. 18B). This finding indicated that the primary mechanism of translocation of ExoS into the cytosol of T24 cells was through secretion by internalized $\mathrm{Pa}$. A shift in the mobility of cytosolic ExoS was observed by SDS-PAGE analysis following co-culture with $\mathrm{Pa}$ expressing ExoSR146A, E381A, or R146A/E381A mutants. This shift related to ExoS auto-ADPRT activity (257) attributed to residual ADPRT activity of the active E379 residue retained by these mutants. The functional significance of this activity remains unclear.

To understand how mutations in ExoS ADPRT activity when combined with an ExoS GAPmutation further enhanced $\mathrm{Pa}$ internalization, IF analyses were performed comparing $\mathrm{Pa}$ internalization by ExoS GAP- and ExoS GAP-/ADPRT- mutants. These analyses revealed that Pa expressing ExoS GAP-/ADPRT- mutants entered and migrated within T24 cells along actin filaments that guided their perinuclear migration (Fig. 18C, indicated by yellow arrows). In 
comparison, $\mathrm{Pa}$ expressing an ExoS GAP- mutant entered T24 cells, but Pa intracellular migration did not align with actin, nor was it generally directed to the perinuclear region (Fig. 18C R146A panels, indicated by blue arrows). The inability of $\mathrm{Pa}$ expressing an ExoS GAPmutant, which retained active E379 and/or E381 ADPRT residues, to align with actin filaments in T24 cells is consistent with ExoS ADPRT activity disrupting actin-plasma membrane associations required for Pa-actin alignment. Enhanced $\mathrm{Pa}$ internalization by ExoS GAPIADPRT- mutants can in turn be related to their ability to align with actin in T24 cells, which secondarily allows increased ExoS translocation by internalized $\mathrm{Pa}$.

To summarize these results, ExoS translocation into cytosol of T24 cells related to secretion of ExoS by internalized $P a$, and enhanced $P a$ internalization and ExoS translocation by $P a$ expressing ExoS GAP-/ADPRT- mutants related to the ability of $P a$ to align with actin filaments within T24 cell, an ability that was interrupted by ExoS ADPRT activity. 
Figure 18. Examining T3S translocation by internalized Pa. A) T24 cells were co-cultured for 2.2-2.5 hr with $\mathrm{Pa}$ expressing WT ExoS, or ExoS with the indicated GAP- and/or ADPRTmutations, or a pUCP vector control. Cells were stained for external or internal $P a$ as in Figure 17 , and internalized $\mathrm{Pa}$ (bar graph) or externally bound $\mathrm{Pa}$ (line graph) were enumerated based on $\mathrm{Pa}$ association with an average of $124 \mathrm{~T} 24$ cells per strain. Results are expressed as the mean \pm SEM of the number of $\mathrm{Pa}$ internalized or externally bound per T24 cell. Asterisks mark significant differences in $\mathrm{Pa}$ internalization $\left({ }^{* *} P<0.001\right.$, A vs. B). A significant difference $(P<0.05)$ in external $P a$ binding was also detected by $P a$ expressing WT ExoS or an ExoSR146A GAP- mutant, as compared to all other Pa ExoS mutant strains (not indicated on graph). Results are representative of six independent experiments. B) T24 cells were co-cultured for 3.5-4 hr with the indicated Pa strains, cells were harvested, fractionated, and ExoS translocation into cytosolic fractions was assayed by immunoblot analysis. RalA, which localizes to the membrane, and GAPDH, which localizes to the cytosol were used as cell fractionation controls. Asterisks mark auto-ADP-ribosylated ExoS attributed to an active E379 ADPRT catalytic residue retained by the respective strains. C) T24 cells were co-cultured from 2.2-3 hr with $\mathrm{Pa}$ expressing the indicated ExoS mutant, and cells were stained for internal or external Pa, ExoS, and actin, as described in Figure 17. Pa expressing ExoS GAP-/ADPRT- mutants aligned along actin filaments (indicated by yellow arrows) consistent with an actin-based motor directing $\mathrm{Pa}$ internalization and migration to the perinuclear region of T24 cells. Pa expressing an ExoSR146A GAP- mutant entered T24 cells but did not align along actin filaments (blue arrows mark internalized $\mathrm{Pa}$ ). White arrows indicate direction of migration polarity. Scale bar is $10 \mu \mathrm{m}$. 


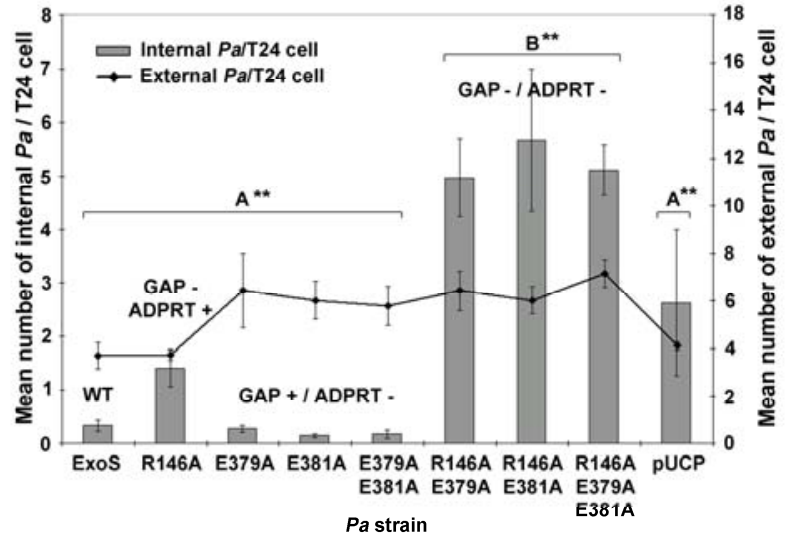

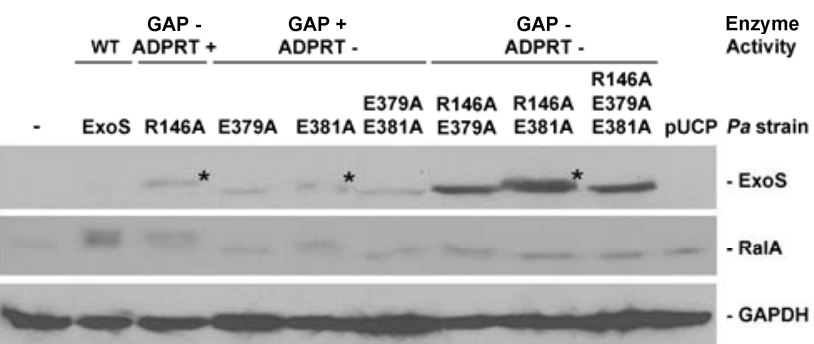

C
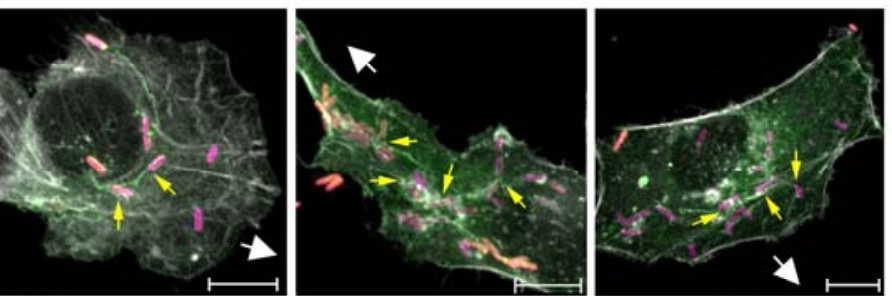

\begin{tabular}{c}
$\begin{array}{c}\text { R146A } \\
\text { E379A }\end{array}$ \\
\hline $\begin{array}{c}\text { GAP - } \\
\text { E381-ADPRT + }\end{array}$
\end{tabular}

E381-ADPRT +
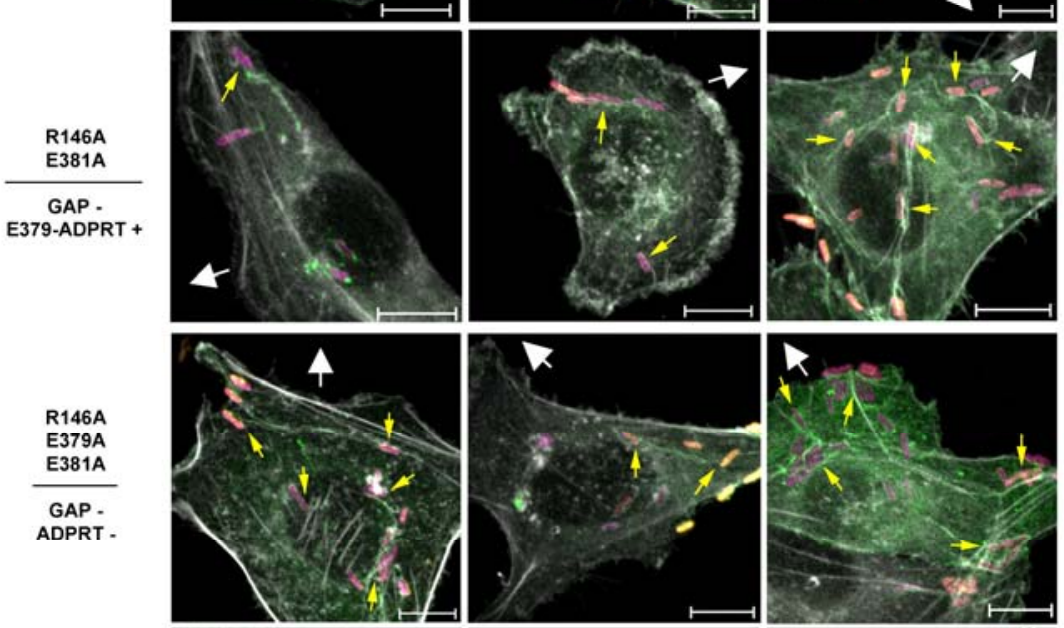

$\frac{\text { R146A }}{\text { GAP - }}$
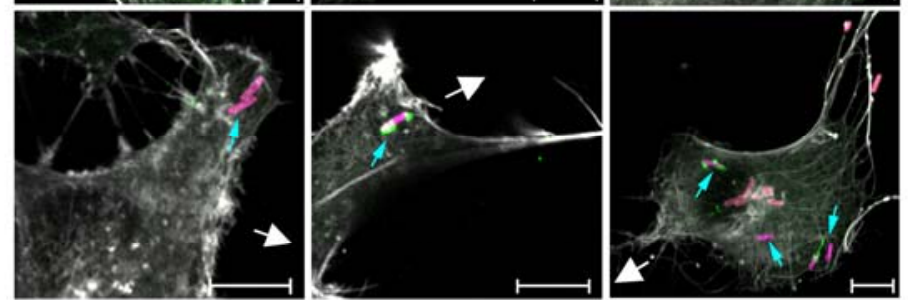
Examining the role of actin in ExoS internalization. Although we were able to establish the mechanism for the negative effects of ExoS GAP and ADPRT activity on T3S translocation, the actual role of the leading edge in T3S translocation remained unclear. Insight into how the leading edge facilitated T3S translocation was obtained during Pa internalization studies, where it was observed in IF analyses that ExoS was tracking actin filaments. The tracking of actin by ExoS is visualized in Figure $18 \mathrm{C}$ in all R146A/E379A mutant panels, the first and second R146A/E381A mutant panel, all R146A/E379A/E381A mutant panels, and the third R146A GAP- mutant panel (this is not specifically marked in Fig. 18C). In fact the tracking of actin by ExoS facilitated detection of internalized $\mathrm{Pa}$ alignment with actin. When ExoS-actin tracking was related to mutations in ExoS enzyme activity, WT ExoS, ExoS GAP-, and/or ExoS ADPRTwere all able to track actin, indicating that actin tracking by ExoS was independent of its enzyme activity (Fig. 19A). However, it should be noted that ExoS-actin tracking was observed more frequently when ExoS ADPRT mutations were introduced.

To further assess ExoS interaction with actin within T24 cells, Pa expressing an ExoS-KDD3XFLAG ExoS GAP-/ADPRT- mutant was used in co-immunoprecipitation (CoIP) studies to identify proteins that interact with T3S translocated ExoS. Four major ExoS interactive protein bands were identified by SDS-PAGE in CoIP analyses (Fig. 19B). When these bands were excised and analyzed by MALDI-MS, band 1, $45 \mathrm{kDa}$, was identified to contain peptides from lysosomal associated protein 1 (LAMP1); band 2, $40 \mathrm{kDa}$, was identified to include peptides from beta-actin; band 3, $30 \mathrm{kDa}$, was identified as the epsilon isoform of 14-3-3 protein; and band 4 was identified to include 14-3-3 proteins of mixed isoforms. The finding that 14-3-3 proteins interact with ExoS is consistent with previous studies (352) and with 14-3-3 proteins being a co-factor of ExoS ADPRT activity (102). Bands 1 and 2 were not homogenous proteins, and the identification of LAMP1 as an ExoS interactive protein in band 1 is consistent with $\mathrm{Pa}$ expressing an ExoS GAP-/ADPRT- mutant being internalized within endocytic vesicles where 
ExoS would have access to lysosomal proteins. Importantly for our studies the co-localization of actin with ExoS in band 2 confirms the ability of T3S translocated ExoS to interact with actin in T24 cells. In turn, ExoS translocation at the leading edge can be explained by this being a site of close actin-plasma membrane association, which would allow T3S translocated ExoS access to actin to facilitate its internalization. 
Figure 19. ExoS aligns with actin filaments in T24 cells. A) $P a$ expressing the indicated ExoS constructs were co-cultured with T24 cells and stained for external $P a$, internal $P a$, ExoS, and actin as in Figure 17. Yellow arrows point to the tracking of ExoS along actin filaments. Scale bar is $10 \mu \mathrm{m}$. B) T24 cells were co-cultured for $4.5 \mathrm{hr}$ with PA103 $\Delta$ UT expressing ExoSKDD-3XFLAG, an ExoS GAP-/ADPRT- inactive mutant (ExoSi) or the pUCP vector control. Coimmunoprecipitation eluates were resolved by SDS-PAGE, silver stained and the indicated bands $\left({ }^{*}\right)$ were excised and analyzed by MALDI-MS. A lysis buffer negative control is also shown. *1: included lysosomal-associated membrane protein-1 (LAMP1), *2: included beta actin, *3: identified as 14-3-3 epsilon, and *4: included 14-3-3 proteins of different isotypes. Results are representative of two independent ColP experiments. 
A

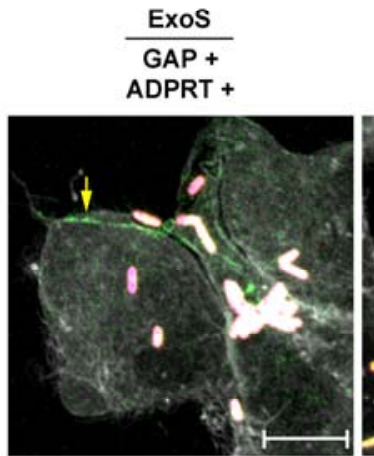

R146A

ADPRT +

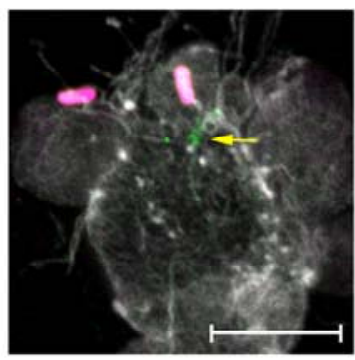

E379A

GAP +

E381-ADPRT +

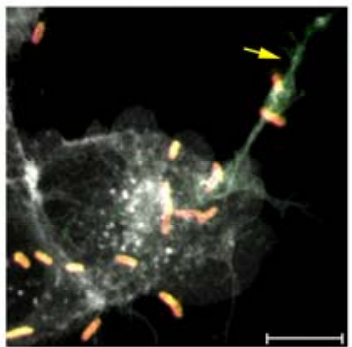

R146A / E379A

GAP -

E381-ADPRT +

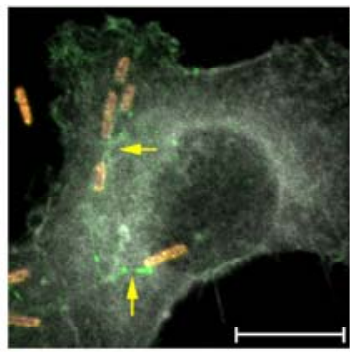

E381A

E379-ADPRT +

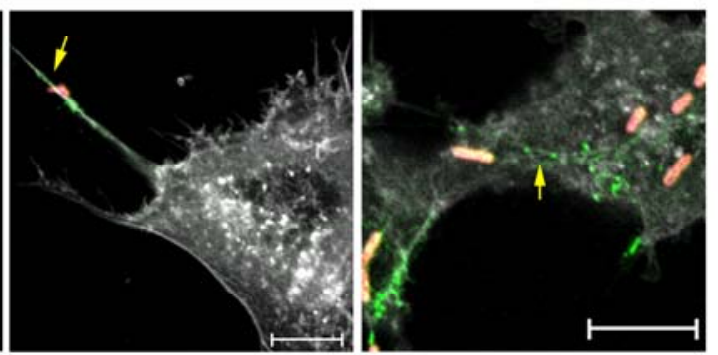

R146A / E381A

GAP -
79-ADPRT +

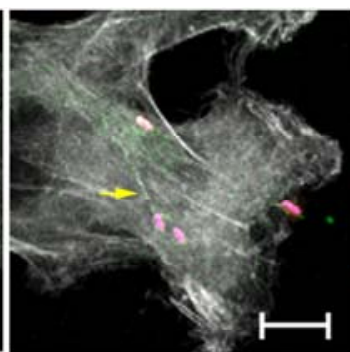

E379A / E381A GAP + GAP +
ADPRT Enzyme Activity

B

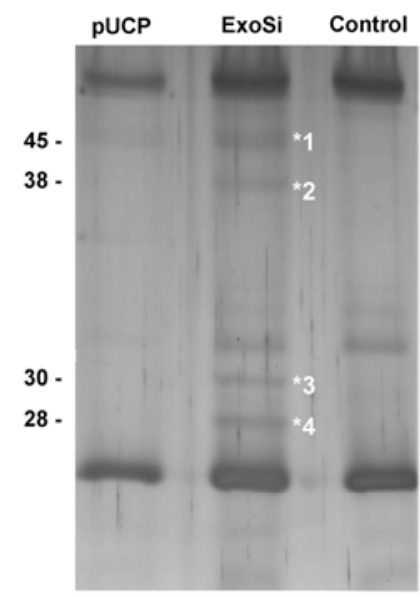


Examining the role of ExoS ADPRT activity in T3S translocation. The finding that mutations in ExoS ADPRT activity enhance ExoS translocation in T24 cells provided evidence that ExoS ADPRT activity targets and inactivates a cellular protein(s) required for T3S translocation. ERM proteins (ezrin, radixin, and moesin) mediate the regulated linkage of actin to the plasma membrane at the leading edge $(32,309)$ and are high affinity substrates of ExoS ADPRT activity (195). Consistent with ERMs being a target of ExoS ADPRT activity that interrupts T3S translocation: 1) ADP-ribosylation of ERMs by ExoS was found to interfere with ERM phosphorylation (pERM) $(195,297)$, which is required for ERM binding to F-actin, and 2) we found ExoS ADPRT activity to disrupt actin anchorage to plasma membrane in conjunction with inhibition of Pa-T3S (Figs. 17B and C, blue arrows) and the alignment of internalized $\mathrm{Pa}$ with actin (Fig. 18C, R146A panels, blue arrows)

When $\mathrm{Pa}$ expressing ExoS mutants were examined for interference of ERM phosphorylation in T24 cells, pERM in membrane fractions was significantly reduced $(P<0.001)$, based on quantification of immunoblot analysis, following co-culture with $\mathrm{Pa}$ expressing ADPRT active ExoS (WT ExoS or an ExoS GAP- mutant), as compared with other ExoS mutant strains (Fig. 20A). Interestingly neither an E379A nor E381A mutation alone caused a decrease in ERM phosphorylation, indicating that mutations in both ADPRT residues were required to interrupt pERM-actin linkage. When pERM and ezrin localization was examined by IF and related to T3S translocation by ExoS mutants, co-localization of pERM and ezrin within leading edge architecture was observed in untreated (0) or pUCP vector control treated T24 cells, confirming localization of pERM to the leading edge in T24 cells (Fig. 20B). This leading edge architecture was lost following co-culture with $\mathrm{Pa}$ expressing WT ExoS, where pERM and ezrin were observed to diffuse away from the leading edge but remain at the cell periphery, sometimes colocalizing to constricted patches. Single ExoS-E379A or E381A ADPRT mutations allowed the effect of each residue on leading edge-pERM architecture to be evaluated. Co-culture with a $\mathrm{Pa}$ 
ExoS-E379A mutant (having an active E381 residue) led to diffuse pERM-ezrin staining along the leading edge, but leading edge architecture remained intact. An ExoS-E381A mutant (having an active E379 residue) led to a loss of leading edge architecture, but ezrin and pERM remained co-localized along the cell periphery. When both ADPRT residues were mutated (Fig. 20B, E379A/E381A panels), leading edge properties and pERM-ezrin staining closely resembled that of uninfected or Pa pUCP treated control cells. The coordinated effects of ExoS E379 and E381 residues on leading edge architecture can be related to ExoS E379 having a more pronounced influence on pERM-ezrin localization to the leading edge, while ExoS E381 had a more pronounced influence on underlying leading edge architecture.

In comparing effects of ExoS ADPRT activity on ERM phosphorylation and Pa-T3S translocation, it was recognized that mutations in both ExoS-E379A and E381A were required to inhibit ERM phosphorylation, while individual ExoS-E379A or E381A mutations were able to enhance T3S translocation. This outcome can be related to the ability of ExoS E379 or E381 to disrupt pERM-ezrin leading edge architecture to interfere with T3S translocation, yet neither residue alone is able to inhibit ERM phosphorylation and disrupt actin-plasma membrane linkages predicted to be required for T3S translocation, thus providing a mechanistic explanation for the coordinated roles of ExoS E379 and E381 in inhibiting T3S translocation. 
Figure 20. Effect of ExoS ADPRT activity on ERM phosphorylation and localization within T24 cells. A) T24 cells were co-cultured for 3.5-4 hr with no bacteria (0), $\mathrm{Pa}$ expressing the indicated ExoS construct or the pUCP vector control. Cells were fractionated and membrane fractions were assayed by immunoblot analysis for pERM (ezrin-Thr567, radixin-Thr564, and moesin-Thr558) and total ezrin. Densitometry analysis detected a significant decrease $(P<0.001)$ in pERM following co-culture with $P a$ expressing WT ExoS or ExoS-R146A GAPmutant, as compared to the other Pa ExoS mutant strains. B) T24 cells were untreated (0) or were co-cultured for $2 \mathrm{hr}$ with $\mathrm{Pa}$ expressing the pUCP vector control or indicated ExoS construct and examined by IF, staining for pERM (pink), ezrin (green), or cell associated $\mathrm{Pa}$ (pink or pink + yellow, indicated by yellow arrows). White arrows indicate direction of migration polarity. Scale bar is $10 \mu \mathrm{m}$. Results are representative of three independent experiments. 


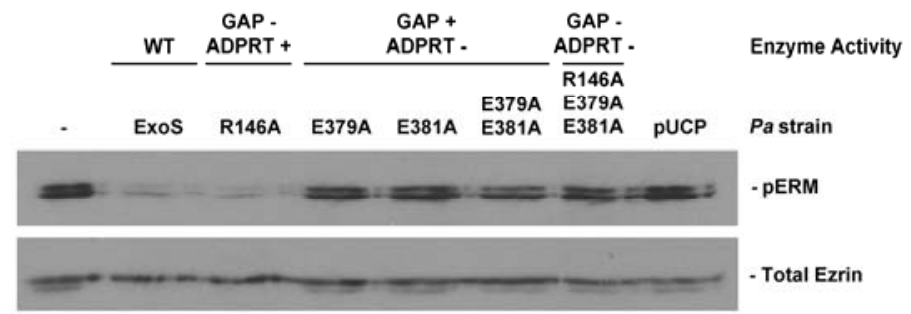

$p E R M+P a$

Ezrin

Merge

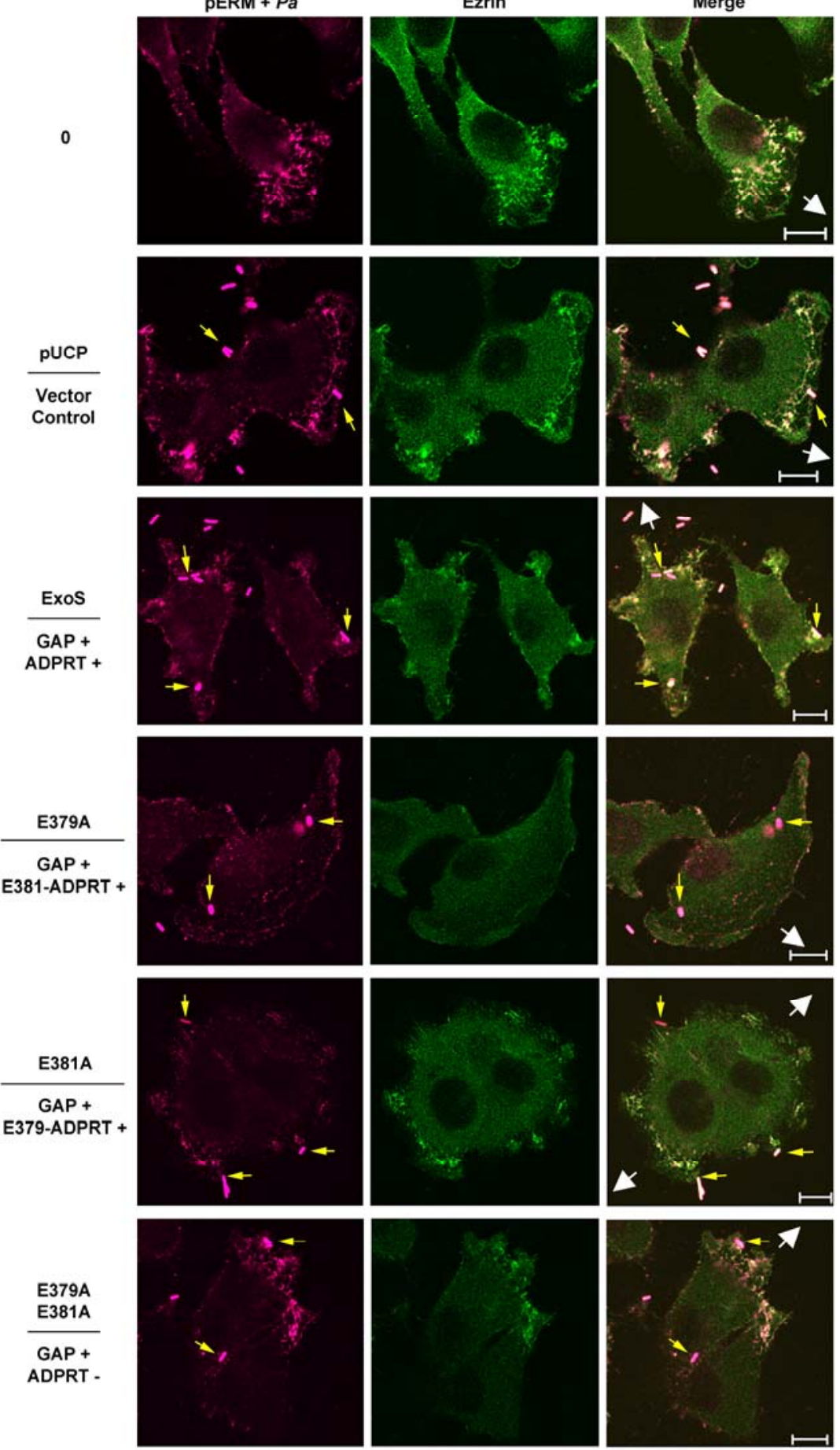




\section{DISCUSSION}

This study addressed the cellular mechanism underlying the establishment of opportunistic $\mathrm{Pa}$ infections by using mutations of ExoS enzyme activity to modulate T3S translocation and the ability of $\mathrm{Pa}$ to infect cells. The study was directed by the hypothesis that actin-plasma membrane associations at the leading edge are required for T3S translocation and evolved by: first, identifying the effects of ExoS GAP and ADPRT activities on T3S in migrating T24 epithelial cells; second, demonstrating that $\mathrm{Pa}$ expressing WT ExoS preferentially bound to the leading edge of T24 cells; third, showing that ExoS ADPRT activity disrupted actin-plasma membrane anchoring in conjunction with interruption of T3S translocation; and fourth, demonstrating that both $\mathrm{Pa}$ and ExoS internalization were facilitated by their ability to align with or track actin. Interestingly, through manipulations of ExoS GAP and ADPRT activities we were also able to unveil the innate infectious potential of $\mathrm{Pa}$ in T24 cells and constraints introduced by ExoS that influence the opportunistic nature of $\mathrm{Pa}$ infections.

The importance of T3S to Pa infections is shown by the ability of ExoS or ExoU to define the invasive or cytotoxic phenotype of $P a$ during infections $(82,88,285)$, and by the ability of the translocon protein, PcrV, to induce protective immunity against $\mathrm{Pa}$ infections (282). $\mathrm{Pa}$ is regarded as an extracellular pathogen, but a small percentage of $\mathrm{Pa}$ are internalized into epithelial cells, a percentage that varies with $\mathrm{Pa}$ strain and with epithelial cell line confluency and polarity $(76,88,243)$. ExoS GAP activity has an anti-internalization function $(15,64,100$, 263), but we found that ExoS ADPRT activity can further enhance the anti-internalization activity ExoS GAP activity in T24 cells. Pa expressing WT ExoS was internalized at a low frequency of an average of one Pa per three T24 cells. This frequency increased 4-fold upon introduction of an ExoS GAP- mutation and 15-fold when both ExoS GAP- and ADPRT- mutations were introduced. IF studies revealed that increased internalization a Pa ExoS GAP-/ADPRT- mutant related to $\mathrm{Pa}$ entering T24 cells and aligning with actin filaments that directed perinuclear 
migration of $\mathrm{Pa}$. The lower level of internalization by a Pa ExoS GAP- mutant coincided with an inability to align with actin after entry into T24 cells; an activity that was lost due to ExoS ADPRT activity retained in this strain. Previous transmission electron microscopy (TEM) studies revealed that $\mathrm{Pa}$ enter cells in tightly enclosed membrane vesicles, which increase in size and eventually lyse as $P a$ traverse inward $(76,89,244)$. We did not examine $P a$ vesicle association within T24 cells, but we observed Pa expressing an ExoS GAP- mutant to be internalized within a membrane vesicle in TEM analysis of J774A.1 macrophages, indicating that this is a common mechanism of internalization, even when an ExoS GAP- mutation is introduced (Supplementary Fig. S7). Also, in IF analysis of T24 cells we were able to visualize intense ExoS staining closely surrounding internalized $\mathrm{Pa}$, which is a staining pattern consistent with $\mathrm{Pa}$ secreting ExoS while contained within a membrane vesicle. Interestingly, this staining pattern was detected by $\mathrm{Pa}$ at the periphery of the lamellipodium, within the lamellipodium, and in the perinuclear regions of T24 cells indicating that ExoS expression was being induced at different stages in $\mathrm{Pa}$ intracellular migration process. ExoS translocation by internalized $\mathrm{Pa}$ was confirmed in cytosolic fractionation and immunoblot analyses. The notion that ExoS is induced after $\mathrm{Pa}$ internalization adds a new level of unknowns to T3S induction mechanisms but is consistent with a recent report describing the translocation of Yops into the host cell cytosol by the Yersinia Ysc T3S system (354).

ExoS GAP and ADPRT activities influenced ExoS T3S translocation in T24 cells at a relative efficiency of WT ExoS < ExoS GAP- < ExoS ADPRT- < ExoS GAP-/ADPRT-, as assessed based on cell population (immunoblot) and single cell (IF) approaches. Subsequent studies allowed characterization of the eukaryotic cell basis for this hierarchy of T3S translocation efficiency. The slight increase in T3S translocation detected by Pa expressing an ExoS-R146A GAP- mutation was attributed to the translocation and sequestering of ExoS within the T24 cells by internalized $\mathrm{Pa}$. Yersinia T3S effector, YopE, functions similarly to ExoS GAP in its ability to 
down-regulate Rho-GTPases, interfere with phagocytosis, and decrease effector translocation $(4,26,207,328)$. However, the mechanism of YopE interference of T3S translocation appears to differ from that of ExoS GAP in being linked to regulatory influences on actin polymerization that affect pore formation, and to the down-regulation of Yop synthesis $(4,5,207,324)$. We were unable to detect an influence of ExoS GAP activity on pore formation in T24 cells, based on trypan blue exclusion assays that compared membrane integrity of $\mathrm{Pa}$ expressing WT ExoS and an ExoS GAP- mutant (Table II). We were also unable to detect a negative impact of ExoS GAP activity on T3S protein expression, based on levels of ExoS and PopB in T24 cell coculture supernatants following infection with $\mathrm{Pa}$ ExoS mutants (Fig. 15D). In this regard, previous studies using A549 epithelial cells and an exoS-GFP reporter construct were able to detect a negative feedback effect of ExoS enzymatic activity on $\mathrm{Pa}$ induction of T3S (45). Differences observed between our studies and those of Cisz et al., may relate to our use of a $\mathrm{Pa}$ population based ExoS expression assay and their use of single cell Pa-T3S induction assay. However, in our system, potential negative feedback effects of ExoS enzyme activity on T24 cell mediated induction of Pa-T3S appear to be secondary to effects of ExoS on host cell properties that influence $\mathrm{Pa}$ binding and T3S translocation. Apparent differences in the mechanism of interruption of T3S translocation by ExoS GAP and YopE relate to some extent to bacterial properties, as demonstrated by the ability of ExoS GAP to complement a YopE mutant in suppressing T3S translocation when expressed in Yersinia (4). It is also possible that influences of ExoS ADPRT activity, which are lacking in Yersinia, may impact the role of ExoS GAP activity on Pa-T3S translocation.

More fundamental to understanding the mechanism underlying T3S translocation was the pronounced inhibition on ExoS translocation caused by ExoS ADPRT activity. This finding supports the novel notion that eukaryotic protein(s) targeted by ExoS ADPRT activity inhibit T3S translocation. ERM proteins link actin to the plasma membrane at the leading edge $(32,309)$ 
and are high affinity substrates of ExoS ADPRT activity (195). Consistent with ERM proteins being targets of ExoS ADPRT activity that interrupt T3S translocation, ExoS ADPRT activity was found to: 1) disrupt actin-plasma membrane linkages hypothesized to be involved in Pa-T3S translocation, and 2) alter leading edge pERM-ezrin architecture in conjunction with decreased T3S translocation. Further evidence for the role of ERMs in Pa-T3S was provided by individual ExoS-E379A or E381A ADPRT mutations, which enhanced Pa-T3S translocation but were individually ineffective in inhibiting ERM phosphorylation. Enhanced T3S translocation can be related to loss of the toxic effects of the individual ExoS E379 or E381 residues on leading edge architecture. Continued T3S translocation in the presence of the remaining active ADPRT residue can also be explained by the inability of either residue alone to inhibit ERM phosphorylation and disrupt actin-plasma membrane linkages predicted to be required to $\mathrm{Pa}$ T3S translocation. The importance of this finding is that it provides a mechanistic explanation for the coordinated roles of ExoS E379 and E381 in inhibiting T3S translocation, which is consistent with the hypothesis that actin-plasma membrane associations at the leading edge underlie PaT3S translocation. While further studies are required to confirm the role of ERM proteins in PaT3S translocation, the prospect of identifying a host cell protein that mediates T3S translocation offers the potential of being applied to the development of novel anti-Pa therapeutic strategies that are resilient to normal antibiotic resistance mechanisms.

In direct comparisons of ExoS and PopB membrane translocation it was also recognized that mutations in ExoS ADPRT activity caused a more pronounced increase in ExoS than PopB translocation (Figs. 15B and C). The bias in the interference of ExoS translocation by ExoS ADPRT was subsequently related to the ability of ExoS to interact with actin filaments within T24 cells, an interaction that would be compromised by toxic effects of ExoS ADPRT activity on actin-plasma membrane linkage. PopB membrane insertion presumably is not dependent on an interaction with actin, and hence its translocation would not be as directly affected by the 
disruption of actin-plasma membrane linkage. The mechanism underlying increased translocation by $\mathrm{Pa}$ ExoS ADPRT- mutants can in turn be related to actin-plasma membrane anchorage at the leading edge and within the lamellipodium that is retained in the absence of ExoS ADPRT activity and allows continued ExoS translocation. The ability of ExoS to bind actin provides a new perspective on adaptations acquired by T3S effectors to facilitate their internalization. Collectively, studies of ExoS ADPRT activity introduced four novel findings to Pa-T3S by: 1) differentiating the mechanism of PopB membrane insertion from that of ExoS translocation; 2) identifying the potential of actin to facilitate ExoS internalization, and 3) recognizing that a target of ExoS ADPRT activity was involved in T3S membrane translocation, and 4) providing a mechanistic explanation consistent with this target being ERM proteins based on studies of individual ADPRT catalytic residues.

The highest levels of ExoS and PopB translocation occurred in association with mutations in both ExoS GAP and ADPRT activities, which can be explained by the different and additive influences of ExoS GAP and ADPRT activities on T3S translocation. In relating our findings of ExoS mutants to the $\mathrm{Pa}$ infectious process, the scenario that evolved was that translocation of WT ExoS into T24 cells occurred initially at the leading edge, where actin is in close association with the plasma membrane and accessible to interaction and tracking by ExoS. Upon translocation, ExoS GAP activity interferes with $P a$ internalization, and ExoS ADPRT activity interrupts actin-plasma membrane associations. Therefore, both activities serve to limit T3S translocation but through different mechanisms. Interestingly, mutation of ExoS GAP and ADPRT activities allowed recognition of the innate ability of $\mathrm{Pa}$ to be internalized, to align along actin filaments, and to continue T3S translocation within cells; a fate that might be encountered by the low percentage of WT Pa that are normally internalized in T24 cells. Our studies also draw attention to the paradoxical role of ExoS in the $\mathrm{Pa}$ infectious process. First, cytotoxic effects of ExoS contribute to the establishment of Pa infections. Second, these same cytotoxic 
effects interfere with $\mathrm{Pa}$ internalization and T3S translocation to limit the $\mathrm{Pa}$ infectious process and help maintain its extracellular, opportunistic lifestyle. The importance of $P a$ remaining extracellular for survival is documented by the redundancy of T3S effectors that have antiinternalization activity, including ExoS GAP, ExoT GAP, ExoT ADPRT, and ExoY (15, 52, 53, $64,66,100,108,109,263)$. Similarly, the integral role of T3S effectors in the opportunistic nature of $\mathrm{Pa}$ infections was recognized in our studies of a PA103 UUT ExoS GAP-/ADPRTmutant, which was found to be efficiently internalized, express high levels of inactive ExoS, remain viable, and cause minimal T24 cell toxicity. This finding unveiled the innate potential of $\mathrm{Pa}$ be an intracellular symbiont; a lifestyle that has been redirected through the acquisition of an array of T3S effectors.

T24 cells have a highly migratory mesenchymal phenotype and were selected for our studies to examine how cell migration properties function in T3S and in the initiation of $\mathrm{Pa}$ infections. Eukaryotic cell properties are known to influence the toxicity of ExoS GAP and ADPRT activities (270), and our findings in T24 cells may differ from those observed in other cell lines. For example, rodent cell lines which have a more limited ExoS ADPRT substrate repertoire and are highly sensitive to negative effects of ExoS GAP activity $(264,270)$, may be less sensitive to negative effects of ExoS ADPRT activity on T3S translocation. Differences have also been observed in ExoS responsiveness among human epithelial cell lines. Recent studies using realtime phase-contrast imaging to examine the effects of ExoS on immortalized corneal epithelial cells found $\mathrm{Pa}$ expressing ADPRT active ExoS to produce membrane blebs that contain replicating $\mathrm{Pa}$ (15), a phenotype not observed in T24 cells. The mechanism underlying cellular differences in ExoS responsiveness between corneal epithelial cells and T24 cells remains unclear, but blebbing is mediated by signaling through Rho, ROCK, and myosin and does not initially involve actin polymerization $(78,99)$. This signaling pathway differs from that at the leading edge of T24 cells, which is Rac1 mediated and involves actin polymerization (99). Rho 
and Rac1 activation are reciprocally balanced within cells, particularly during migration (275). Previous studies of MDCK cells found that $\mathrm{Pa}$ internalization was stimulated by RhoA activation, a property that was lost when MDCK cells acquired apical-basolateral polarity $(155,156)$. These findings introduce the possibility that increased internalization and $\mathrm{Pa}$ survival in corneal epithelial cells may relate to properties linked to Rho activation that are absent at the leading edge of T24 cells. Our future plans include examining how the balance between Rho and Rac1 activation at the leading edge of corneal epithelial and T24 cells directs the outcome of Pa-T3S responsiveness.

To summarize, as an opportunist, $\mathrm{Pa}$ infections are influenced by host cell properties $(45,206$, 271). Through the ability to visualize ExoS translocation in relation to T24 cell migration we were able to characterize mechanisms underlying differences in T3S translocation caused by mutations in ExoS GAP and ADPRT activities. These analyses led to the recognition of a role of host cell actin in both ExoS and $\mathrm{Pa}$ and internalization, a role that was disrupted by ExoS ADPRT activity. Our study was directed by the hypothesis that actin-plasma membrane associations at the leading edge of migrating cells are required for T3S translocation and contribute to the establishment of $\mathrm{Pa}$ infections. While this remains a hypothesis, it provided direction for studies that led to new understanding of opportunistic Pa infections. 


\section{ACKNOWLEDGEMENTS}

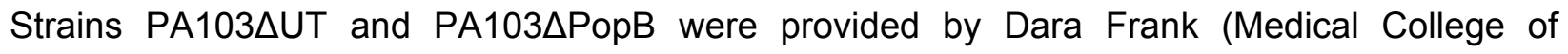
Wisconsin, Milwaukee, WI). ExoS-HA and R146K/E379D/E381D-3XFLAG were provided by Joseph Barbieri (Medical College of Wisconsin, Milwaukee, WI). We would like to acknowledge the assistance of Robert Wysolmerski and Kimberly Arnold in the development of immunofluorescent protocols. Imaging experiments and image analysis were performed in the West Virginia University Imaging Facility, which is supported in part by the Mary Babb Randolph Cancer Center and NIH grant P20 RR016440. We would also like to acknowledge the assistance of Carol Moskos (Pathology Department, Medical University of South Carolina) in the transmission electron microscopy studies. Statistical analyses were performed by Gerry Hobbs (West Virginia University Department of Statistics). These studies were funded in part through grant P20 RR016477 from the National Center for Research Resources, awarded to the West Virginia IDeA Network for Biomedical Research Excellence. We are grateful to Slawomir Lukomski, Matthew Novotny, and the Infection and Immunity reviewers for their helpful comments on this manuscript. 

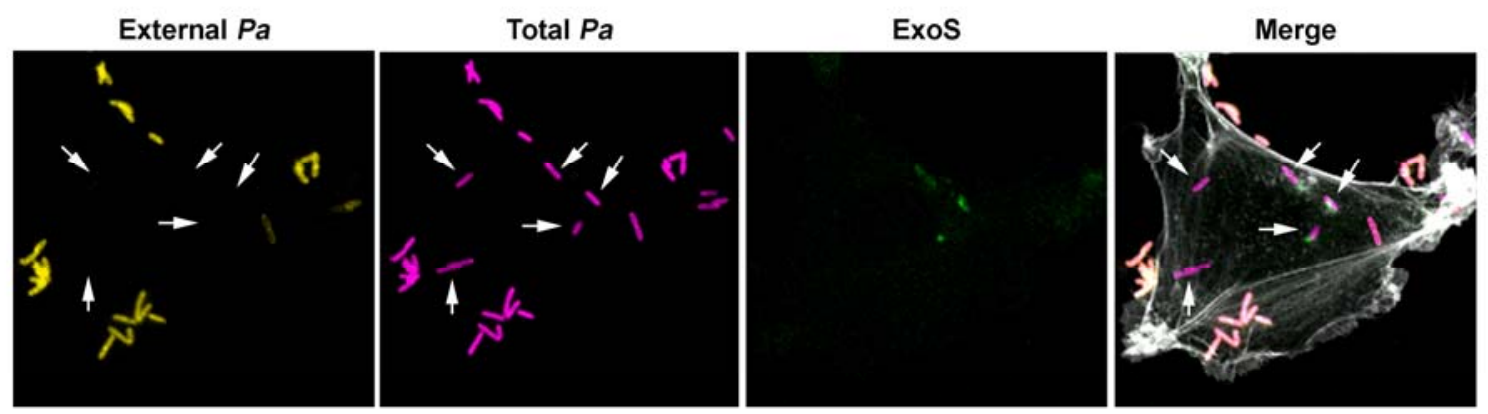

Supplementary Figure S6. Staining for external Pa, internal Pa, and ExoS expression. T24 cells were co-cultured for $2.2 \mathrm{hr}$ with PA103DUT expressing an ExoS-R146A/E379A/E381A GAP-/ADPRT- mutant. Following co-culture, cells were washed, fixed in $3 \%$ paraformaldehyde in actin stabilization buffer, and blocked. External $\mathrm{Pa}$ (yellow + pink in merged image) were detected using rabbit anti-Pa LPS and a Qdot 655 anti-rabbit conjugate. Cells were fixed again, permeabilized, and blocked. Total $\mathrm{Pa}$ were detected using anti-Pa LPS followed by an Alexa Fluor 647 conjugate (pink). ExoS expression was detected using an anti-HA antibody and an Alexa Fluor 488 conjugate (green). Actin was stained with TRITC-phalloidin (white). Intracellular Pa marked by white arrows were identified by the absence of Qdot 655 staining. 
Supplementary Figure S7. Visualizing Pa internalization within membrane vesicles by transmission electron microscopy. J774.1 murine macrophage-like cells (ATCC TIB-67) were: A) untreated, or co-cultured for $2.5 \mathrm{hr}$ with strain PA103 UUT expressing B) WT ExoS or C-D) an ExoS-R146A GAP- mutant. After the co-culture period, cells were washed, fixed with $2 \%$ cacodylate glutaraldehyde, then post-fixed with $2 \%$ aqueous osmium tetraoxide. Samples were rinsed with distilled water, dehydrated with $100 \%$ ethanol, and embedded. Thin sections (70 nm) were cut, placed on copper grids, and double stained with uranyl acetate and lead citrate. Samples were examined with a JEOL JEM 1010 transmission electron microscope. Red arrows point to $\mathrm{Pa}$. C) Shows $\mathrm{Pa}$ internalized in a membrane vesicle, which is enlarged in $\mathbf{D}$ ). Fuzziness at the upper right end of the vesicle in $\mathbf{D}$ ) is interpreted to reflect membrane lysis. Increased vacuole formation was observed in association with ExoS ADPRT activity of WT ExoS and the ExoS-R146A GAP mutant-. Images are representative of two independent analyses. Magnification is indicated for each image. 


\section{A Control}

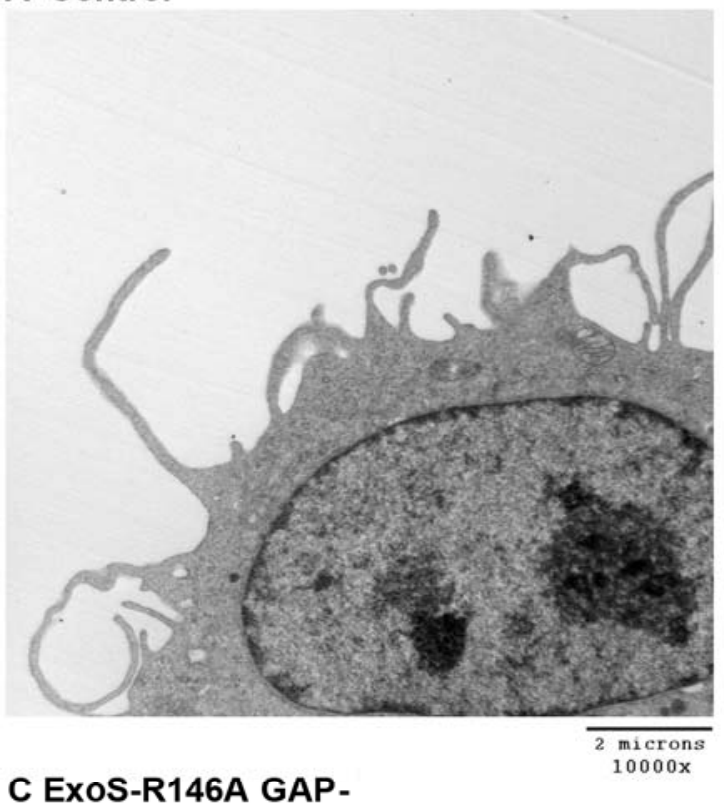

C ExoS-R146A GAP-

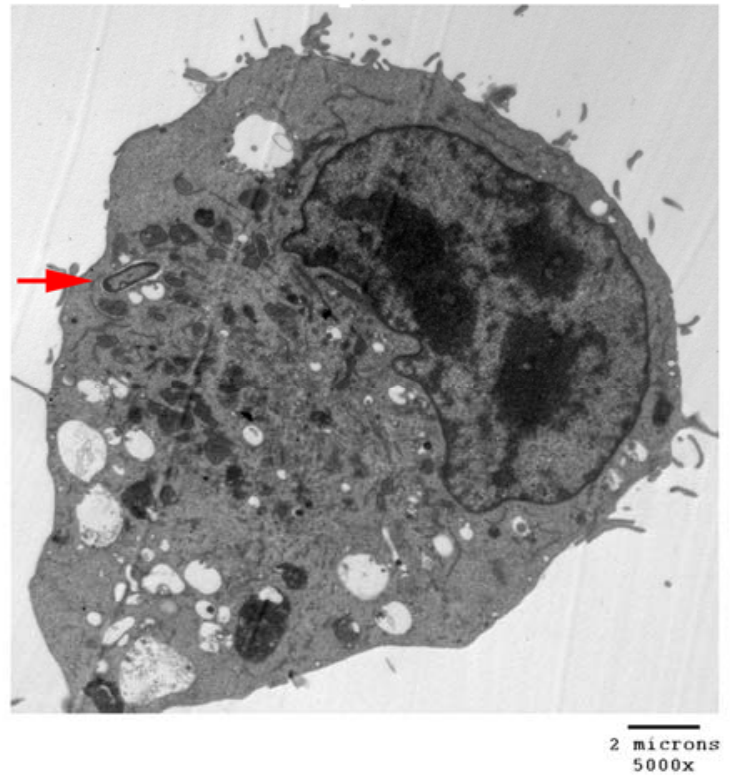

B WT ExoS
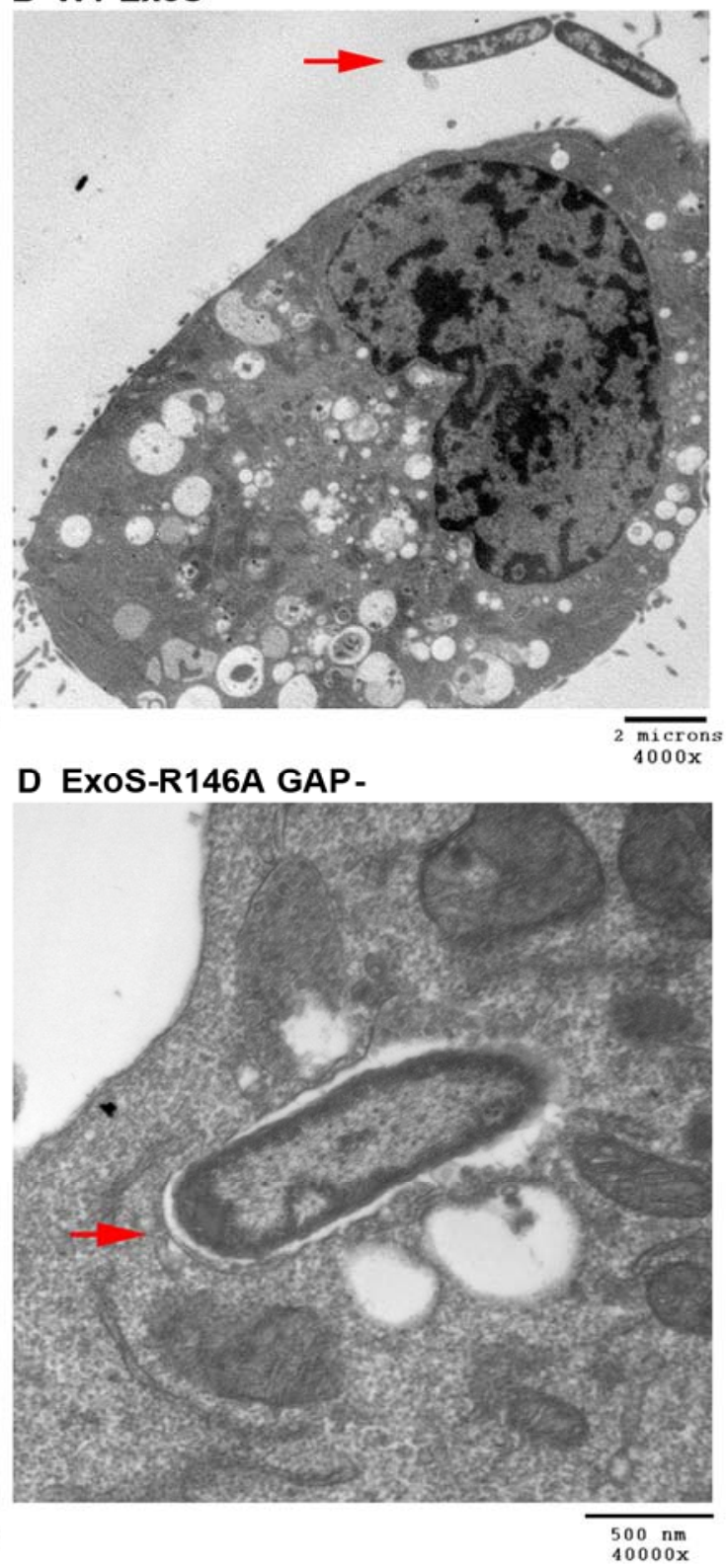


\section{Chapter 4: Differences in Host Cell Migration Properties Lend Insight into Pseudomonas aeruginosa Type III Secretion Susceptibility}

The MTC and MTLn3 experiments were performed in conjunction with Matthew J. Novotny. MTC and MTLn3 experimental results presented in this chapter will be combined with experiments performed by Matthew J. Novotny for publication. Matthew J. Novotny and Dacie R. Bridge will be co-first authors on the manuscript. 


\section{INTRODUCTION}

Eukaryotic cell migration is an essential process used by all nucleated cells and is characterized as either individual or collective. Individually migrating cells can move by forming membrane blebs, which is also known as amoeboid migration, or by forming distinct leading and trailing edges, also known as mesenchymal migration. Cells utilizing amoeboid migration tend to be poorly adhesive to the extracellular matrix (ECM), form membrane blebs, and are driven by Rho/Rho-Kinase (ROCK) regulated propulsion at the leading edge $(178,349)$. In contrast, cells migrating via a mesenchymal phenotype can be highly adhesive, have high contractility, and propulsion is mediated by Rac1 activity at the leading edge balanced by Rho in the trailing edge $(121,227,305,338)$. Collective migration is a process by which multiple cells coordinate leading edge protrusions with simultaneous retraction of the cell rear. While the process resembles mesenchymal migration, cells migrating collectively maintain contact with each other. As a result, the cells move as a sheet, which occurs during wound healing $(7,98,133,305)$. Leading edge dynamics in migrating cells are controlled by Rho-GTPases. In the classical model of cell migration, Rac1 mediates lamellipodial protrusion while Cdc42 establishes cellular polarity and mediates filopodial protrusions $(221,277)$. Rho in turn, mediates actomyosin retraction of the trailing edge of the cell, and maintains cellular adhesion to the ECM (221). Rac1 and Rho have been shown to work in a reciprocal fashion where increased Rac1 down-regulates Rho and vice versa (276). This balance between Rac1 and Rho signaling is essential in signaling cells to migrate via an amoeboid or mesenchymal mechanism (273).

Previous studies from our laboratory have highlighted the importance of the leading edge of migrating cells and Rac1/Cdc42 signaling in host cell susceptibility to Pseudomonas aeruginosa-type III secretion (Pa-T3S) (33)(Chapter3). T3S is a virulence mechanism utilized by numerous Gram-negative pathogens such as Yersinia, Salmonella, Shigella, Escherichia coli, and $\mathrm{Pa}$. $\mathrm{Pa}$ is an opportunistic pathogen that is known to target individuals with wounded 
epithelial barriers, individuals who are immunocompromised, and individuals with have cystic fibrosis. Unlike obligate pathogens, $\mathrm{Pa}$ is able to target and establish infection in any cell type. Several studies have implicated a role for Pa-T3S in the establishment of infection, thereby illustrating its importance as a $\mathrm{Pa}$ virulence factor (reviewed in (129)). The T3S system translocates $\mathrm{Pa}$ effector proteins, such as ExoS, directly from the bacterial cytosol into the host cell, thus bypassing the host immune response. Translocation of ExoS across the eukaryotic membrane requires channel formation by the hydrophobic proteins PopB and PopD (215). PcrV, the hydrophilic Pa-T3S needle tip protein is required for stabilization of the translocon channel in the host cell membrane (215).

Several studies have indicated that the host cell response to $\mathrm{Pa}$ infection depends largely on the cell type studied $(33,89,206,270,271)$. In studies of over 40 cell lines, most were identified as being sensitive to Pa-T3S and infection (206). Two cell lines have been identified as resistant to Pa-T3S, polarized epithelial monolayers and promyelocytic HL-60 cells $(206,271)$. Both cell lines could be induced to become T3S-sensitive either through disruption of epithelial cell apical-basolateral polarity, or by differentiation of HL-60 cells with the phorbol ester TPA (206, 271). However, despite this knowledge, the common factor underlying host cell sensitivity to PaT3S remains unknown.

The purpose of the current study was to determine how Rho-GTPases and their role in host cell migration influence Pa-T3S. We have previously proposed that actin polymerization and linkage to the membrane, together with the role of Rac1/Cdc42 mediated focal complex formation at the leading edge of migrating cells are the host cell compromise resulting in susceptibility to T3S and the establishment of $\mathrm{Pa}$ infection ((33), studies presented in Chapter 3$)$. Therefore, to further investigate the hypothesis that host cell migration is involved in host cell sensitivity to PaT3S, we chose three cell lines which exhibit differences in Rho-GTPase mediated cell migration patterns. Rat mammary adenocarcinoma MTC and MTLn3 cell lines are a clonal cell model 
system that differs in metastatic potential where MTC cells exhibit low metastatic potential and MTLn3 cells exhibit high metastatic potential $(70,217)$. There are several advantages to using MTC cells and its MTLn3 clonal sub-line for the current studies. First, both cell lines have an identical genetic background with little cellular heterogeneity (54). Second, this model system focuses solely on the differences in metastatic potential (54). Third, MTC cells are known to exhibit more polarized cell migration, and can move faster than MTLn3 cells (292). Finally, host cell migration in MTC and MTLn3 cells differs in Rho-GTPase regulation of leading edge cell migration properties $(71,72)$. In MTC cells, lamellipodial protrusions are Rac1-dependent and levels of activated Rho and total Rho are decreased, consistent with the classical model of cell migration. However, in MTLn3 cells, the reciprocal balance between Rho and Rac1 influences lamellipodial protrusion. El-Sibai et al. have previously shown that Rho activation in the lamellipodia of MTLn3 cells is increased, and as a result, Rac1 activity at the leading edge is suppressed and lamellipodial protrusion is Cdc42-dependent $(71,72)$. Madin-Darby canine kidney (MDCK) cells have largely been used to understand the wound healing process and polarization of epithelial cells $(17,83,200)$. In this regard, MDCK cells can migrate collectively during wound healing or can use mesenchymal migration to migrate individually (246). When wounding is initiated in an MDCK monolayer, cell migration is responsible for wound closure and not cell proliferation $(268,299)$. Wound closure also depends on Rac1, phosphoinositide, cJun, and ERK1/2 MAP kinase $(11,83,200)$. While Rho is not directly required for wound healing, it is required for the formation of F-actin bundles in areas along the wounded edge, which aid in even healing and in formation of membrane ruffles $(83,173)$.

Our hypothesis for these studies was that differences in host cell migration properties, based on activated Rho-family GTPases at the leading edge, influences sensitivity to Pa-T3S. Studies utilizing metastatic MTLn3 cells found that increased Pa-T3S sensitivity, compared to nonmetastatic MTC cells, was attributed to Pa internalization due to MTLn3 cell resistance to ExoS 
GTPase activating protein (GAP) activity. In wounded monolayer studies utilizing MDCK cells, sensitivity to Pa-T3S was greatest 4-6 hr post-wounding. ExoS was found to inhibit wound healing through its enzyme activity, where ExoS GAP activity inhibited actin stress fiber formation while ExoS ADP-ribosyltransferase (ADPRT) activity inhibited ERM (ezrin, radixin, and moesin) phosphorylation and actin attachment to the membrane. Together, both activities contributed to disruption of cell migration through inhibition of actin stress fiber formation required for cell migration and wound healing. Overall, these studies support the hypothesis that host cell properties regulating leading edge dynamics influence susceptibility to Pa-T3S, in that cells expressing high levels of Rho at the leading edge are more resistant to Pa-T3S. The identification of host cell migration as a contributing or initiating factor in the establishment of $\mathrm{Pa}$ infection is consistent with the opportunistic lifestyle of $\mathrm{Pa}$, which is known to target compromised epithelial barriers. 


\section{MATERIALS AND METHODS}

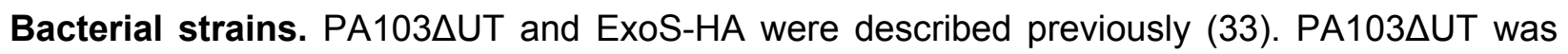
used to express plasmid encoded enzymatic mutants of ExoS, shown in Table I (Chapter 3). Pa were grown in ExoS induction medium (147) for 14-16 hr and were washed twice in cell line specific medium prior to co-culture with eukaryotic cells.

Eukaryotic cell culture and bacterial eukaryotic cell co-culture conditions. MTC cells (nonmetastatic) were obtained from John S. Condeelis (Albert Einstein College of Medicine, Bronx NY). MTLn3 cells (metastatic) were obtained from Scott Weed (West Virginia University School of Medicine, Morgantown WV). MTC and MTLn3 cells were cultured in Minimum Essential Medium, Alpha (aMEM; Cellgro, Manassas VA) containing nonessential amino acids (Cellgro), $10 \%$ fetal bovine serum (FBS; Hyclone, Logan UT), $100 \mathrm{U} / \mathrm{ml}$ penicillin, and $100 \mu \mathrm{g} / \mathrm{ml}$ streptomycin (Hyclone). MTC and MTLn3 cells were seeded at $2 \times 10^{4}$ and $2.5 \times 10^{4}$ cells $/ \mathrm{ml}$, respectively, and grown for $48 \mathrm{hr}$. Prior to bacterial co-culture, cells were washed twice with Dulbecco's phosphate buffered saline (DPBS; Hyclone) and starved for $3 \mathrm{hr}$ in aMEM containing 0.35\% bovine serum albumin (BSA; Sigma-Aldrich, St. Louis MO) and 12 mM HEPES (pH 7.4; starvation medium). $\mathrm{Pa}$ were added at $10^{7} \mathrm{CFU} / \mathrm{ml}$ (224) in aMEM containing $5 \%$ FBS (stimulation medium) (292) and co-cultured with either cell line for 3-4 hr.

Madin-Darby Canine Kidney NBL-2 (MDCK) cells were obtained from American Type Cell Culture Collection (ATCC, Manassas VA) and were cultured in Eagles Minimum Essential Medium (EMEM; ATCC) with 10\% FBS, $100 \mathrm{U} / \mathrm{ml}$ penicillin, and $100 \mu \mathrm{g} / \mathrm{ml}$ streptomycin (Hyclone). MDCK cells were seeded at $0.4 \times 10^{5}$ cells $/ \mathrm{ml}$ and grown for $48-72 \mathrm{hr}$. MDCK cells were co-cultured with $0.5 \times 10^{7} \mathrm{CFU} / \mathrm{ml} \mathrm{Pa}$ or no bacteria for the indicated time as previously described (224). 
Drug inhibitor studies. Following the $3 \mathrm{hr}$ starvation, MTC and MTLn3 cells were treated for 30 min with $25 \mu \mathrm{M}$ Y-27632 (ROCK inhibitor) (Cayman Chemical Company, Ann Arbor MI) or for 12-16 hr with $50 \mu \mathrm{M}$ NSC23766 (Rac1 inhibitor) (EMD Chemicals, Gibbstown, NJ) in starvation medium. Cells were co-cultured with $\mathrm{Pa}$ strains in the presence of inhibitor as previously described. The effects of the drugs on cell function were visualized with an LD A-Plan 20x/0.03 Ph1 objective on a Zeiss Axiovert 200 microscope (Jena, Germany). Images were exported to Adobe Photoshop CS4 Extended (Adobe Systems Inc., San Jose, CA) as TIFF files and cropped.

Analysis of T3S translocation. MTC, MTLn3, and MDCK cells were seeded in $60 \mathrm{~mm}$ dishes (Corning Inc., Corning NY) and grown for $48 \mathrm{hr}$ to $70-90 \%$ confluency. Following a 3.5-5 hr coculture, bacteria were removed and cells were washed twice in DPBS (Hyclone) with $200 \mu \mathrm{g} / \mathrm{ml}$ ciprofloxacin. Adherent cells were detached with $0.05 \%$ trypsin-1 mM EDTA (Hyclone). An aliquot of cells was analyzed for total protein (BCA Protein Assay; Pierce, Rockfield IL). In MTC and MTLn3 studies, remaining cells were resuspended in DPBS containing ciprofloxacin and lysed with Laemmli sample buffer (174). In MDCK cell studies, remaining cells were resuspended in digitonin fractionation buffer, and fractionated as previously described (264). Glyceraldehyde 3-phosphate dehydrogenase (GAPDH) served as a loading control for MTC and MTLn3 cell lysates. In MDCK studies, total RalA was used as a membrane fraction loading control and GAPDH was used as a cytosolic fraction loading control.

SDS-PAGE and immunoblot detection of the T3S effector ExoS-HA constructs were used to monitor T3S translocation. ExoS ADP-ribosylation of RalA served as a functional read-out of translocated ExoS enzyme activity (96). Antibodies used in immunoblot studies include: monoclonal anti-HA (Covance Research Products, Princeton NJ) to detect ExoS-HA constructs, monoclonal anti-RaIA (BD Transduction Laboratories; San Jose CA), monoclonal anti-GAPDH (Millipore, Temecula CA), and anti-phospho-ERM (pERM, Cell Signaling, Danvers MA). For all 
studies, translocation of ExoS-HA into whole cell lysates (MTC and MTLn3 studies) or membrane fractions (MDCK studies) were quantified by densitometry analysis using ImageJ 1.40 software (http://rsbweb.nih.gov/ij/). Quantified results are expressed as the mean relative densitometry intensity, averaging four to six independent experiments.

\section{Immunofluorescence analysis of T3S effector translocation and Pa internalization.}

(i) Non-metastatic vs. metastatic studies. MTC and MTLn3 cells were seeded at $2.4 \times 10^{4}$ cells/ml in chamber slides (Nalge Nunc International, Rochester NY) 24 and 48 hr, respectively, prior to co-culture. Cells were starved for $3 \mathrm{hr}$ as previously described and were co-cultured with the indicated $\mathrm{Pa}$ strain in stimulation medium for $1 \mathrm{hr} 45 \mathrm{~min}, 2 \mathrm{hr} 15 \mathrm{~min}$, or $3 \mathrm{hr}$.

(ii) Wounded monolayer studies. MDCK cells were seeded at $1.4 \times 10^{5}$ cells $/ \mathrm{ml}$ in chamber slides (Nalge Nunc), and cultured for $72 \mathrm{hr}$, which allowed for formation of a confluent monolayer. Cells were washed twice with EMEM with $10 \%$ FBS and a linear wound was initiated with a beveled pipette tip. Following wounding, cells were washed three times with EMEM containing 10\% FBS (109). Wounds were allowed to heal for $0-14 \mathrm{hr}$ prior to a $4 \mathrm{hr}$ coculture with the indicated $\mathrm{Pa}$ strain.

Following co-culture, all cells were washed twice in DPBS (Hyclone), fixed in 3\% paraformaldehyde in actin stabilization buffer (ASB) $(33,115)$, and blocked with $0.8 \%$ BSA (Sigma-Aldrich), $0.1 \%$ fish gelatin, and $10 \mu \mathrm{g} / \mathrm{ml}$ goat $\mathrm{lgG}$. External $\mathrm{Pa}$ were detected using polyclonal anti-Pa LPS (Joseph Lam, University of Guelph, Ontario), followed by blocking with 6\% BSA in DPBS (Hyclone), and visualized using a Qdot 655 goat anti-rabbit conjugate (Invitrogen, Carlsbad CA). Cells were then fixed, permeabilized with $0.2 \%$ Triton $\mathrm{X}-100$ in ASB, and blocked as above. All Pa were stained with rabbit anti-Pa LPS followed by an Alexa-Fluor 647 conjugate (Invitrogen). Internal Pa were differentiated based on the absence of Qdot 655 staining. T3S secreted ExoS constructs were detected using anti-HA (Covance Research 
Products) coupled with an anti-mouse Alexa-Fluor 488 conjugate (Invitrogen). F-actin was stained using tetramethylrhodamine B isothiocyanate conjugated to phalloidin (TRITCphalloidin, Sigma-Aldrich). Bacterial localization and internalization were visually enumerated.

Cells were mounted using Fluormount-G (Southern Biotechnology Associates, Birmingham, $A L$ ) and were visualized with an EC Plan-Neofluar 40x/1.30 oil objective or a Plan-Apochromat $63 x / 1.40$ oil objective on a Zeiss Imager.Z1 LSM 510 confocal microscope (Jena, Germany). Images were exported to Adobe Photoshop CS4 Extended (Adobe Systems Inc., San Jose, CA) as 8 bit TIFF files and cropped.

Statistical analysis. JMP Version 9 Software (SAS Institute, Cary, NC) was used for statistical analyses and significance was determined by a two-sided alpha level set at $P<0.05$. One way ANOVAs were used to evaluate differences among $P a$ strains between cell lines. Multiple comparisons were analyzed with a Tukey's HSD test for ExoS translocation and $\mathrm{Pa}$ internalization. A T-test was used to evaluate comparisons for ROCK and Rac1 inhibitor studies. 


\section{RESULTS}

Relationship between host cell migration properties and sensitivity to Pa-T3S. Initial investigations into cell migration differences showed that MTC cells migrated by a mesenchymal phenotype and contained migrational polarity, which was indicated by the presence of distinct leading and trailing edges (Fig. 21A, left panel). In MTC cells, actin stress fibers were present through the length of the cell and terminated at the leading and trailing edges (Fig. 21A, left panel, blue arrows). In comparison, MTLn3 cells did not exhibit defined migrational polarity as indicated by the broad leading edge and lamellipodium, which allows the cell to migrate in any direction (Fig. 21A, right panel). MTLn3 cells contained low levels of actin stress fibers, which tended to terminate and form an actin band behind the lamellipodium (Fig. 21A, right panel, blue arrows). Most MTLn3 cells did not exhibit a distinct trailing edge. Migrating MDCK cells in a wounded monolayer had a different pattern of cell migration than both MTC and MTLn3 cells. MDCK cells migrated as a collective unit where some of the outermost cells extended broad lamellipodia into the wound (Fig. 21B, left panel, arrowheads). Thick actin bundles were observed along the marginal edge of the wound (Fig. 21B, left panel, arrows). Cells closest to the wounded edge displayed a more elongated phenotype than cells behind the wounded edge, and most migrating cells exhibited high levels of thick actin stress fibers throughout the cell (Fig. 21B, right panel, arrows). In comparison to MTC cells, MDCK cells exhibited a more extensive actin stress fiber network (Figs. 21A and B).

Once differences in host cell migration properties were characterized, host cell sensitivity to PaT3S was determined for the three cell lines. MTLn3 cells were found to be more sensitive to PaT3S than MTC cells as indicated by greater levels of ExoS translocation into MTLn3 cells at a 3 hr time-point (Fig. 21C). Interestingly, if the co-culture proceeded to $4 \mathrm{hr}$, the level of ExoS translocation in MTC cells was comparable to MTLn3 cells indicating that MTC cells are more resistant to the effects of ExoS early in infection (M. J. Novotny, data not shown). Sub-confluent 
MDCK cells were also found to be sensitive to Pa-T3S (Fig. 21C). ExoS translocation was detected two hours later in the MDCK cells than in the MTLn3 cells, and approximately one hour later than in MTC cells. Together these results indicate that between the three cell lines, MTLn3 cells are the most sensitive to Pa-T3S while sub-confluent MDCK cells are the least sensitive. 
Figure 21. Cell line differences in sensitivity to Pa-T3S. A-B) The actin cytoskeleton and overall cell migrational polarity differs between MTC, MTLn3, and MDCK cells. A) MTC cells migrate via mesenchymal migration patterns with distinct leading and trailing edges. MTLn3 cells do not exhibit cell migration polarity as indicated by the broad leading edge and lack of a defined trailing edge. Blue arrows indicate actin stress fibers for both cell lines. B) Left panel, wounded MDCK cells migrated via collective migration. Blue arrowheads indicate cells extending broad lamellipodia into the wound. Blue arrows indicate thick marginal actin bundles along the wounded edge. Right panel, migrating MDCK cells contained numerous actin stress fibers throughout the cell (blue arrows). Cells are stained with TRITC-phalloidin to detect actin (white). White arrows indicate the direction of cell polarity. Scale bar is $10 \mu \mathrm{m}$. C) MTC and MTLn3 cells were treated with strain PA103AUT expressing plasmid encoded WT ExoS for 3$3.5 \mathrm{hr}$ and levels of ExoS translocation into whole cell lysates were evaluated by immunoblot analysis. RalA modification served as a functional read-out of translocated ExoS ADPRT activity. GAPDH served as a loading control. Sub-confluent MDCK cells were treated with strain PA103 $\Delta$ UT expressing plasmid encoded WT ExoS for $5 \mathrm{hr}$. Cells were harvested, fractionated, and T3S translocation of ExoS into the membrane fraction was analyzed by immunoblot. ADPribosylated RalA served as a functional readout of ExoS ADPRT activity and total RalA served as a membrane fraction loading control. Asterisks mark ADP-ribosylated RalA. Results are representative of four to six independent experiments. 


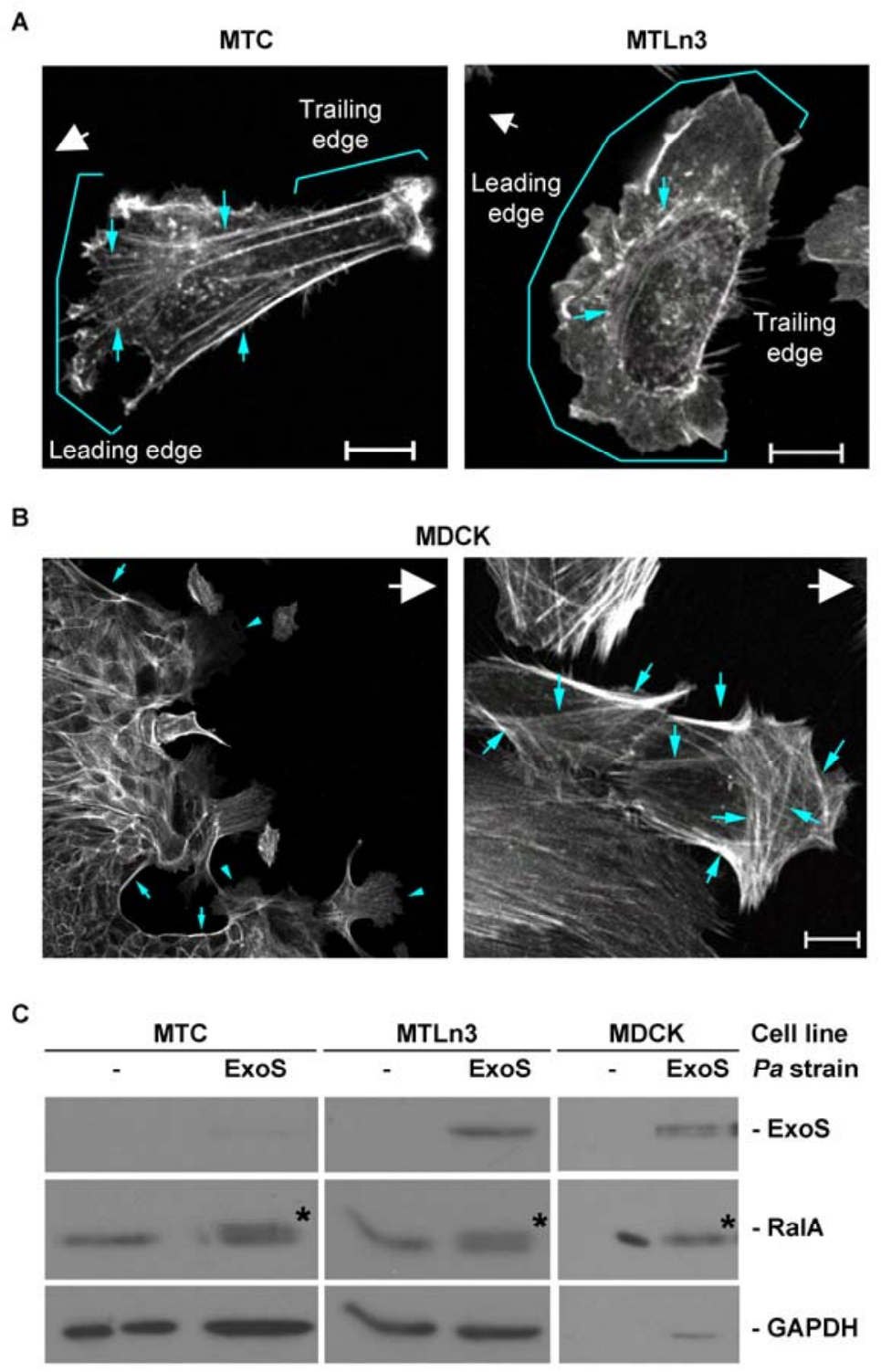


Utilizing ExoS enzymatic mutants to examine differences in Pa-T3S sensitivity. Effects of ExoS enzyme activity on host cell properties are known to influence $\mathrm{Pa}$-T3S responsiveness (Chapter 3) $(4,45,95)$. Therefore, we used ExoS with mutations known to abolish its GAP (R146A) or ADPRT (E379A/E381A) activities as tools to help differentiate host cell properties involved in T3S translocation in the three different cell lines. When MTC cells were treated with the ExoS ADPRT- mutant, an increase in ExoS translocation was observed compared to translocation by $\mathrm{Pa}$ expressing WT ExoS and was further increased with an ExoS GAP- mutant (Fig. 22A). In contrast, an increase in ExoS translocation in MTLn3 cells was only observed when treated with the ExoS GAP- mutant.

When sub-confluent MDCK cells were treated with Pa expressing either the ExoS GAP- mutant or the ExoS ADPRT- mutant, we observed increases in ExoS translocation into the membrane fraction when compared to translocation by $\mathrm{Pa}$ expressing WT ExoS; however, these increases did not reach statistical significance (Fig. 22B). In comparison, when MDCK cells were treated with an ExoS GAP-/ADPRT- (R146A/E379A/E381A) mutant, a significant increase $(P<0.0001)$ in translocation was observed as compared to WT ExoS, the ExoS GAP- mutant, and the ExoS ADPRT- mutant. Together these results indicate that differences in MTC and MTLn3 Pa-T3S sensitivity relates to a target of ExoS GAP activity. While this same pattern was not observed with MDCK cells, an increase in translocation associated with mutation of both ExoS GAP and ADPRT activities is consistent with different host cell properties influencing T3S in MDCK cells. 
A

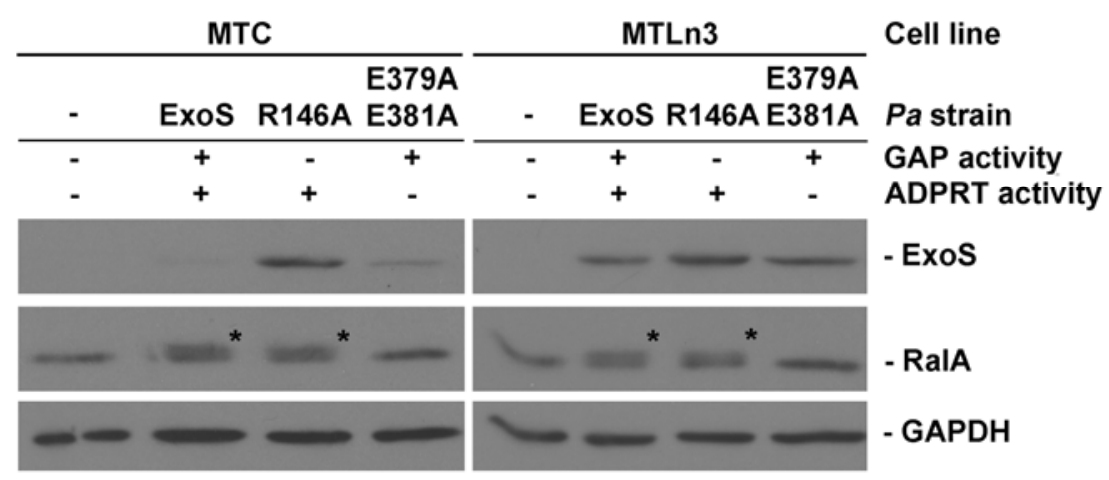

B

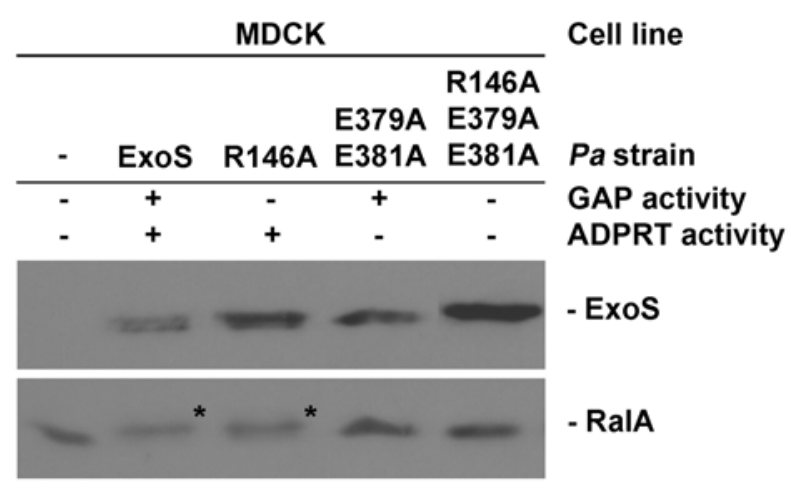

Figure 22. Using ExoS GAP and ADPRT mutants to dissect cell line differences. A) MTC and MTLn3 cells were treated with PA103AUT expressing plasmid encoded ExoS or ExoS with mutations in GAP (R146A) or ADPRT (E379A/E381A) activities for $3.5 \mathrm{hr}$ and whole cell lysates were analyzed by immunoblot. GAPDH served as a membrane fraction loading control. ADPribosylated RalA is indicated by asterisks. B) MDCK cells were treated for $5 \mathrm{hr}$ with PA103 UUT expressing plasmid encoded ExoS or ExoS with mutations abolishing GAP (R146A), ADPRT (E379A/E381A), or GAP/ADPRT (R146A/E379A/E381A) activities. Cells were harvested, fractionated, and T3S translocation of ExoS into MDCK membrane fractions was analyzed by immunoblot. ADP-ribosylated RalA is indicated by asterisks. Total RalA served as a membrane fraction loading control. Results are representative of three to four independent experiments. 
Characterizing differences in the effects of ExoS GAP activity in MTC and MTLn3 cells. In order to understand how Rho-GTPases influenced ExoS translocation in MTC and MTLn3 cell lines, cells were treated with Pa expressing WT ExoS, an ExoS GAP- mutant, or an ExoS ADPRT- mutant and infection was monitored by immunofluorescence and confocal (IF) microscopy. When MTC cells were treated with Pa expressing WT ExoS, the bacteria remained primarily extracellular and low levels of ExoS translocation was detected (Fig. 23A). As MTC cells rounded due to ExoS translocation, a branched actin phenotype was observed. In comparison, Pa expressing WT ExoS were internalized by MTLn3 cells (Fig. 23A, yellow arrows). We also observed an increase in ExoS translocation in MTLn3 cells by IF microscopy studies when compared to MTC cells (Fig. 23A). When both MTC and MTLn3 cells were treated with $\mathrm{Pa}$ expressing the ExoS GAP- mutant, an increase in internalized $\mathrm{Pa}$ was observed as compared to cells treated with Pa expressing WT ExoS (Fig. 23B, yellow arrows). The increased $\mathrm{Pa}$ internalization was associated with an increase in ExoS GAP- mutant translocation. Finally, in both cell lines, the number of internalized $\mathrm{Pa}$ expressing an ExoS ADPRT- mutant was not different from Pa expressing WT ExoS (Fig. 23C, yellow arrows). There was an observed increase in ExoS translocation in MTC cells treated with the ExoS ADPRT- mutant as compared to WT ExoS (Fig. 23C). However, there was no detectable difference in the level of ExoS translocation into MTLn3 cells by $\mathrm{Pa}$ expressing the ExoS ADPRT- mutant when compared to $\mathrm{Pa}$ expressing WT ExoS (Fig. 23C). A branched actin phenotype in the MTC cells was again observed when they were treated with the ExoS ADPRTmutant, which was not observed in the MTLn3 cells treated with the same strain (Fig. 23C).

The percent of $\mathrm{Pa}$ internalized per cell line from IF microscopy studies was quantified for all $\mathrm{Pa}$ strains. When MTC cells were treated with $P a$ expressing WT ExoS, $3 \%$ of $P a$ were internalized (Fig. 23D, gray bars). A significant increase $(P<0.001)$ in $P a$ internalization $(29.4 \%)$ was observed when MTC cells were treated with an ExoS GAP- mutant. MTC cells internalized 5.2\% 
of $\mathrm{Pa}$ expressing an ExoS ADPRT- mutant, which was not significantly different from internalization of Pa expressing WT ExoS (Fig. 23D, gray bars). In comparison, MTLn3 cells treated with $\mathrm{Pa}$ expressing WT ExoS internalized $15.5 \%$ of bacteria (Fig. 23D, blue bars). Pa internalization $(42 \%)$ was significantly increased $(P<0.005)$ when MTLn3 cells were treated with an ExoS GAP- mutant (Fig. 23D, blue bars). As was observed in MTC cells, there was no difference in MTLn3 internalization (15.3\%) of Pa expressing an ExoS ADPRT- mutant when compared to Pa expressing WT ExoS (Fig. 23D, blue bars). Internalization of Pa expressing WT ExoS by MTLn3 cells was significantly increased $(P<0.02)$ compared with MTC cells.

To determine if $\mathrm{Pa}$ internalization by MTLn3 cells was contributing to the increase in ExoS translocation, we quantified ExoS translocation from immunoblot studies by densitometry (Fig. 23E). In MTC cells, a low level of ExoS translocation was observed when cells were treated with Pa expressing WT ExoS or an ExoS ADPRT- mutant. ExoS translocation in MTC cells was significantly increased $(P<0.0001)$ when cells were treated with $P a$ expressing an ExoS GAPmutant (Fig. 23E, gray bars). When MTLn3 cells were treated with $\mathrm{Pa}$ expressing an ExoS GAP- mutant, a significant increase in ExoS translocation was observed when compared to cells treated with Pa expressing WT ExoS or an ExoS ADPRT- mutant (Fig. 23E, blue bars). Translocation of WT ExoS or the ExoS ADPRT- mutant was significantly increased $(P<0.001)$ in MTLn3 cells as compared to translocation into MTC cells (Fig. 23E). Together, these results show that the increase in ExoS translocation in MTLn3 cells by $\mathrm{Pa}$ can be attributed to $\mathrm{Pa}$ internalization and continued ExoS translocation by the bacteria within the cell. 
Figure 23. Increased MTLn3 sensitivity is attributed to internalized Pa. MTC and MTLn3 cells were treated with PA103 $\triangle$ UT expressing plasmid encoded A) WT ExoS, or ExoS with mutations that abolished B) GAP (R146A), or C) ADPRT (E379A/E381A) activities for $3 \mathrm{hr}$. Cells were fixed and stained for external $\mathrm{Pa}$ (yellow + pink), internal $\mathrm{Pa}$ (pink, yellow arrows), ExoS (green), and actin (white). Scale bars represent $10 \mu \mathrm{m}$. White arrows indicate direction of cellular polarity. D) Internal $\mathrm{Pa}$ were quantified and are represented as the percent of internal $\mathrm{Pa}$ relative to total $\mathrm{Pa}$. The asterisks indicate: internalization of $\mathrm{Pa}$ is significantly increased by ${ }^{*}$ MTC cells $(P<0.001)$ and ${ }^{* *}$ MTLn3 $(P<0.005)$ expressing the ExoS GAP- mutant compared to WT ExoS and the ExoS ADPRT- mutant strains. ${ }^{* * *}$ Internalization of $P a$ expressing WT ExoS was significantly increased $(P<0.02)$ in MTLn3 cells as compared to MTC cells. E) Quantification of ExoS translocation by PA103AUT expressing plasmid encoded ExoS or ExoS with mutations abolishing GAP (R146A) or ADPRT (E379A/E381A) activities. Increases in ExoS translocation paralleled increases in $\mathrm{Pa}$ internalization (refer to $\mathrm{D}$ ). Asterisks indicate: ExoS translocation by the GAP- mutant was significantly increased in ${ }^{*} \mathrm{MTC}$ cells $(P<0.0001)$ and ${ }^{* *} \mathrm{MTLn} 3$ cells $(P<0.01)$ compared to $P a$ translocation of WT ExoS or the ExoS ADPRT- mutant; Translocation of ExoS by $P$ a expressing **WT ExoS $(P<0.0001)$ or ${ }^{* * * *}$ the ExoS ADPRT- mutant $(P<0.001)$ into MTLn3 cells was significantly increased compared to MTC cells. Results are representative of four to six independent experiments. 
A

MTC

ExoS

GAP + ADPRT +

MTLn3

B

R146A

GAP -

ADPRT +

MTLn3

C

\begin{tabular}{lr} 
& \\
& MTC \\
& \\
E379A & \\
E381A & \\
\hline GAP + & \\
ADPRT - & \\
& MTLn3
\end{tabular}

Exos
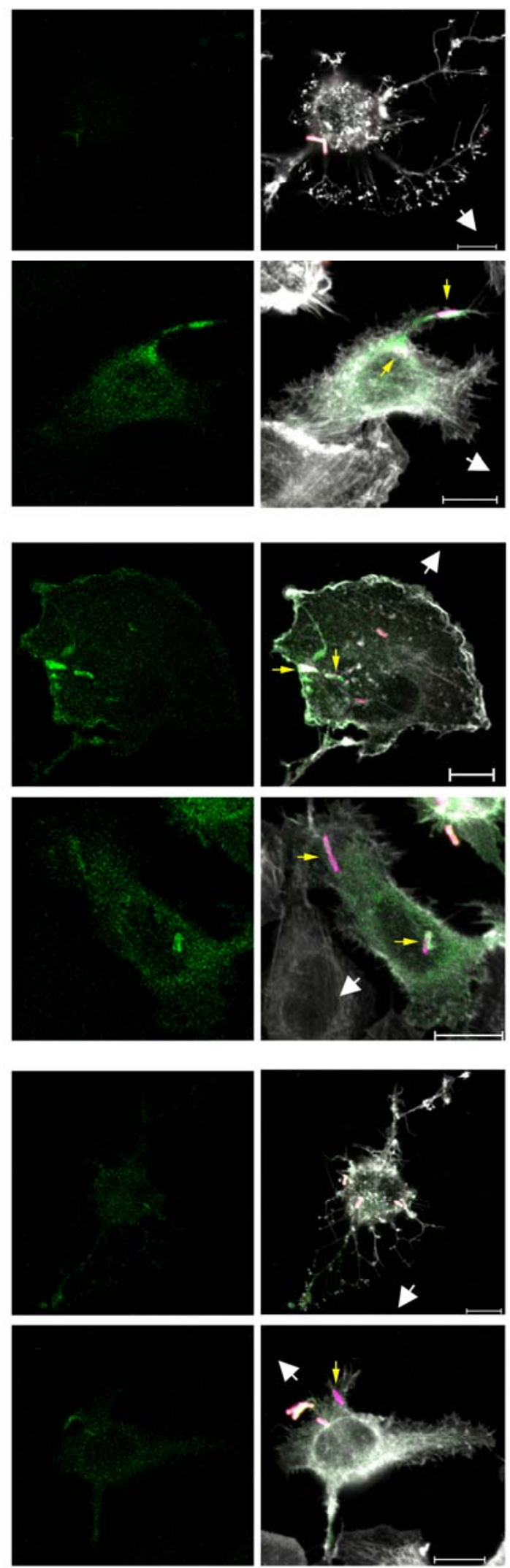
D

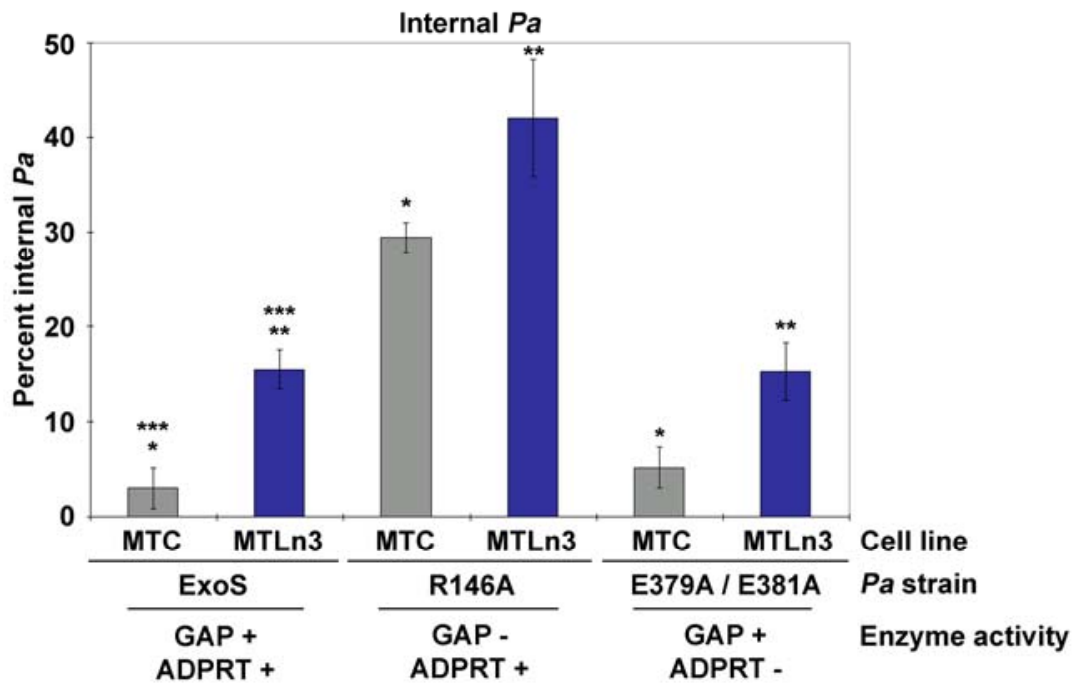

E

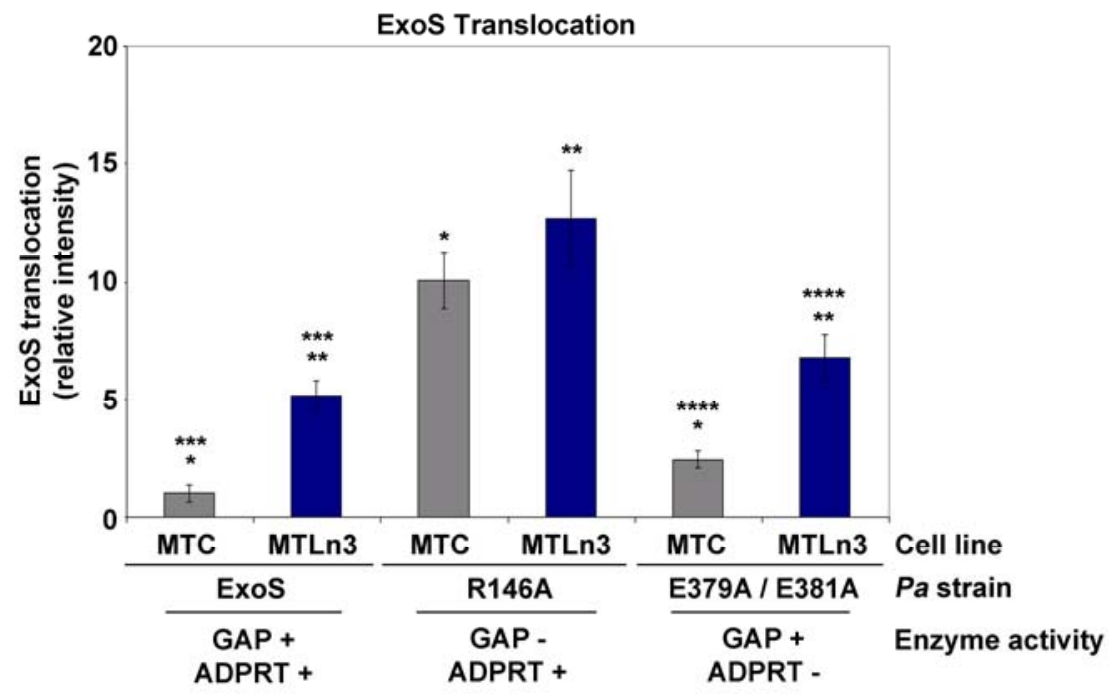


When MTC cells treated with an ExoS GAP active strain (WT ExoS or the ExoS ADPRTmutant) were examined by IF microscopy, a branched actin phenotype was observed as the cells rounded (Figs. 23A and C). To investigate the role of Rho-GTPases and ExoS GAP activity in this phenomenon, MTC and MTLn3 cells were examined at two time-points by IF microscopy. After $1 \mathrm{hr} 45 \mathrm{~min}$ of co-culture, MTC cells treated with $\mathrm{Pa}$ expressing WT ExoS or an ExoS ADPRT- mutant displayed a branched actin phenotype that became more pronounced at $2 \mathrm{hr}$ 15 min (Figs. 24A and C). In MTC cells treated with Pa expressing an ExoS GAP- mutant, there were no major cell morphological changes at either time-point as both the lamellipodium and actin stress fibers remained intact (Fig. 24B).

In comparison, at $1 \mathrm{hr} 45 \mathrm{~min}, \mathrm{~Pa}$ expressing WT ExoS or the ExoS ADPRT- mutant treated MTLn3 cells exhibited a loss of the broad lamellipodium and the band of actin stress fibers that forms behind the lamellipodium (Figs. 24D and F). As MTLn3 cells rounded, short, branched actin projections were observed. While this phenotype was more pronounced at $2 \mathrm{hr} 15 \mathrm{~min}$, it was not as extensive as the branching observed in MTC cells at the $1 \mathrm{hr} 45 \mathrm{~min}$ time-point. After examination at both time-points, the lamellipodium of MTLn3 cells treated with an ExoS GAP mutant was observed to be contracting. Interestingly, the band of actin stress fibers behind the lamellipodium remained intact and the few actin stress fibers, which previously terminated at the actin band, now projected out past the edge of the lamellipodium. These longer actin spikes became more pronounced at $2 \mathrm{hr} 15 \mathrm{~min}$ (Fig. 24E).

To summarize the studies thus far, these results suggest that ExoS GAP activity is targeting Rac1 in MTC cells. Disruption of Rho mediated stress fibers in MTC cells by ExoS GAP activity is consistent with contraction of the lamellipodium if Rac1 is disrupted. In addition, activated Rac1 in the lamellipodia of MTC cells appears to be more sensitive to ExoS GAP activity at early time-points and may function to limit ExoS translocation. MTLn3 cells appear to be more resistant to ExoS GAP activity increased Rho at the leading edge. The limited morphological 
changes in MTLn3 cells when treated with an ExoS GAP active strain may be due to targeting of Rho at the leading edge. To test this hypothesis, the interplay between Rho and Rac1 at the leading edge were investigated. 

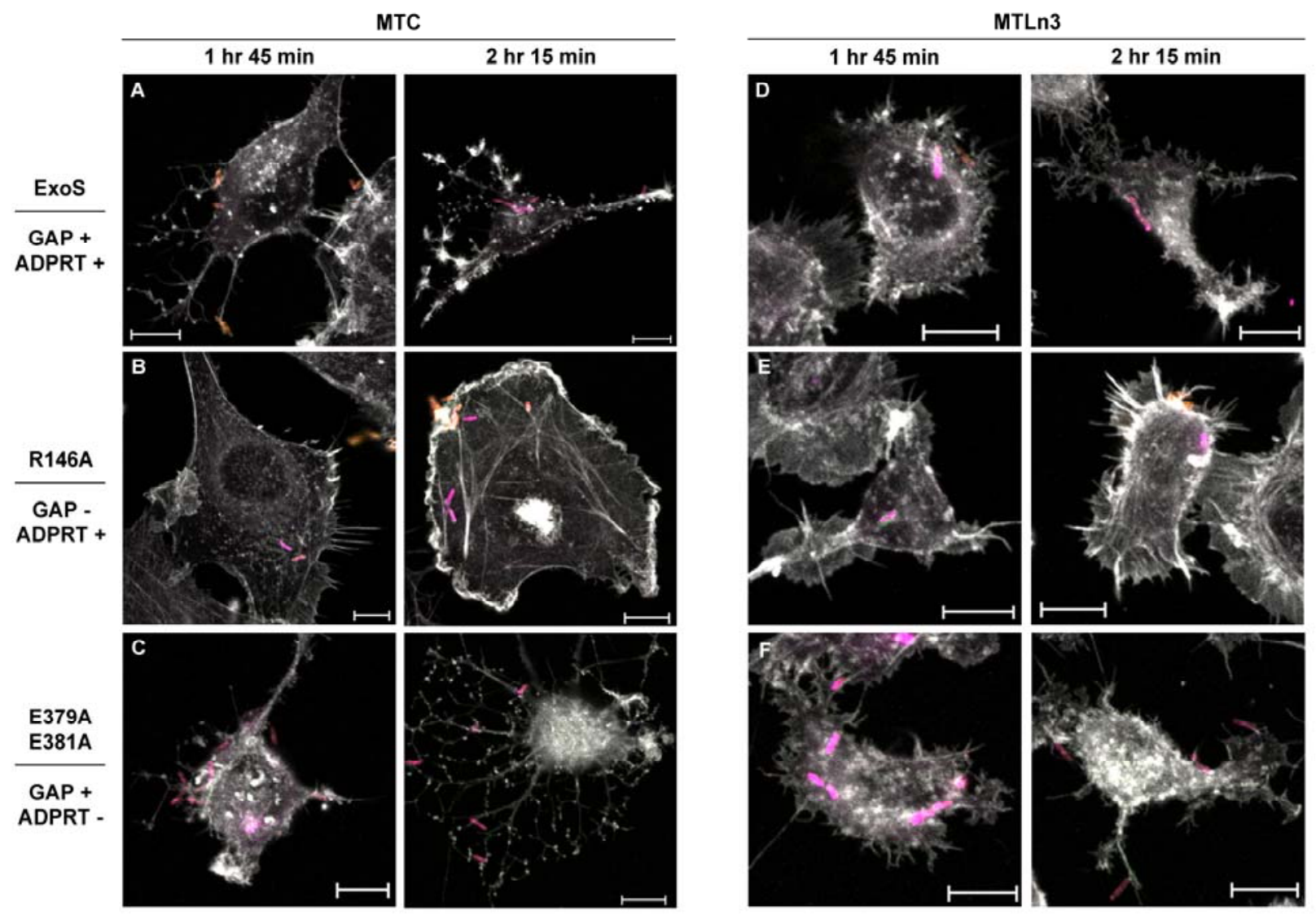

Figure 24. ExoS GAP activity alters Rho-GTPase mediated cell morphology differently in MTC and MTLn3 cells. MTC and MTLn3 cells were treated with Pa expressing: A/D) WT ExoS, B/E) an ExoS GAP- mutant (R146A), or C/F) an ExoS ADPRT- mutant (E379A/E381A) for $1 \mathrm{hr}$ $45 \mathrm{~min}$ or $2 \mathrm{hr} 15 \mathrm{~min}$. Cells were fixed and stained for external $\mathrm{Pa}$ (yellow + pink), internal $\mathrm{Pa}$ (pink), ExoS (green), and actin (white). Scale bar is $10 \mu \mathrm{M}$. Results are representative of one to two independent experiments for each time-point. 
Interplay between Rho and Rac1 in MTC and MTLn3 cells. Previous studies have shown that that the balance between Rho and Rac1 at the leading edge of MTLn3 cells could be shifted by inhibiting Rho/ROCK with the ROCK inhibitor Y-27632 (72). Results from these studies indicated that the high level of activated Rho at the leading edge of MTLn3 cells suppressed Rac1 function. As a consequence, inhibition of ROCK resulted in an increase in Rac1 at the leading edge (72). We hypothesized that the balance between Rho and Rac1 at the leading edge influenced cell sensitivity to Pa-T3S in that if MTC cells were sensitive to ExoS GAP activity due to increased Rac1 at the leading edge, then increasing the level of activated Rac1 in the MTLn3 cells with the ROCK inhibitor would reduce Pa-T3S sensitivity to the level found in MTC cells. In turn, if Rac1 was inhibited in the MTC cells, then MTC cell sensitivity to Pa-T3S would resemble the level of sensitivity observed in the MTLn3 cells. Rac1 inhibition in MTLn3 cells and Rho inhibition in MTC cells was proposed not to have an effect on T3S sensitivity due to the already decreased levels of activated Rac1 and Rho in those cells lines, respectively.

Figure 25 shows phase contrast images of both MTC and MTLn3 cells post-inhibitor treatment. Untreated MTC cells displayed a very limited leading edge (panel A, black arrows). Following starvation conditions, MTC lamellipodial protrusions disappeared (panel B, white arrows). MTC cells treated with either the ROCK inhibitor (panel C) or the Rac1 inhibitor (panel D) exhibited a rounded phenotype. Upon stimulation with 5\% FBS, lamellipodial protrusion was restored regardless of inhibitor treatment (not shown). The lamellipodial protrusions in untreated MTLn3 cells are not detectable by phase contrast microscopy due to their flat, thin structure (panel E). Post-starvation, no major MTLn3 morphological changes were observed (panel F). Interestingly, upon MTLn3 cell treatment with the ROCK inhibitor Y-27632, the lamellipodial protrusions resembled that of untreated MTC cells (compare panel G black arrowheads with black arrows in panel A). Again, no major morphological changes were observed in MTLn3 cells treated with the 
Rac1 inhibitor (panel H). FBS stimulation resulted in a restoration of lamellipodial protrusion in some MTLn3 cells regardless of inhibitor treatment (not shown).

Next, we sought to determine the role of the inhibitors in altering Pa-T3S sensitivity. To evaluate the role of the GAP activity on host cell sensitivity to Pa-T3S, MTC and MTLn3 cells were first treated $25 \mu \mathrm{M}$ Y-27632 to inhibit ROCK followed by co-culture with Pa expressing WT ExoS, the ExoS GAP- mutant, or the ExoS ADPRT- mutant. Following Y-27632 treatment, there was no significant effect on Pa-T3S sensitivity in MTC cells with any of the Pa strains tested (Fig. 26A). However, following treatment of MTLn3 cells with Y-27632, there was a significant decrease in ExoS translocation by $\mathrm{Pa}$ expressing WT ExoS $(P<0.003)$ or the ExoS ADPRT- mutant $(P<0.001)$ to levels of translocation observed in the MTC cell line (Fig. 26; compare light gray bars from part B with part A). There was no significant change in ExoS translocation levels in MTLn3 cells treated with the ROCK inhibitor and the ExoS GAP- mutant (Fig. 26B). To evaluate the role of Rac1 in Pa-T3S sensitivity, MTC and MTLn3 cells were treated with NSC23766. Treatment of MTC cells resulted in an increase in ExoS translocation by all strains tested; however, the increase failed to reach significance (Fig. 27A). There was no effect on ExoS translocation when MTLn3 cells were treated with the Rac1 inhibitor in association with any of the Pa ExoS strains (Fig. 27B).

In summary, these results support the hypothesis that differential sensitivity to Pa-T3S by MTC and MTLn3 cells lines relates to the interplay between Rho-GTPases at the leading edge of migrating cells. Resistance of MTLn3 cells to ExoS GAP activity is proposed to be due to increased levels of activated Rho at the leading edge, which facilitates bacterial internalization and continued effector translocation within the cells. On the other hand, activated Rac1 at the leading edge in MTC cells appears to be sensitive to ExoS GAP activity; therefore the bacteria remain extracellular and translocate limited amounts of effector into the cell, which in turn serves to regulate ExoS translocation. In both cell lines, the role of ExoS ADPRT activity 
appears to be minor compared to ExoS GAP activity, as no substantial increase in translocation or internalization was observed upon mutation of both ADPRT catalytic residues when compared to Pa expressing WT ExoS. 

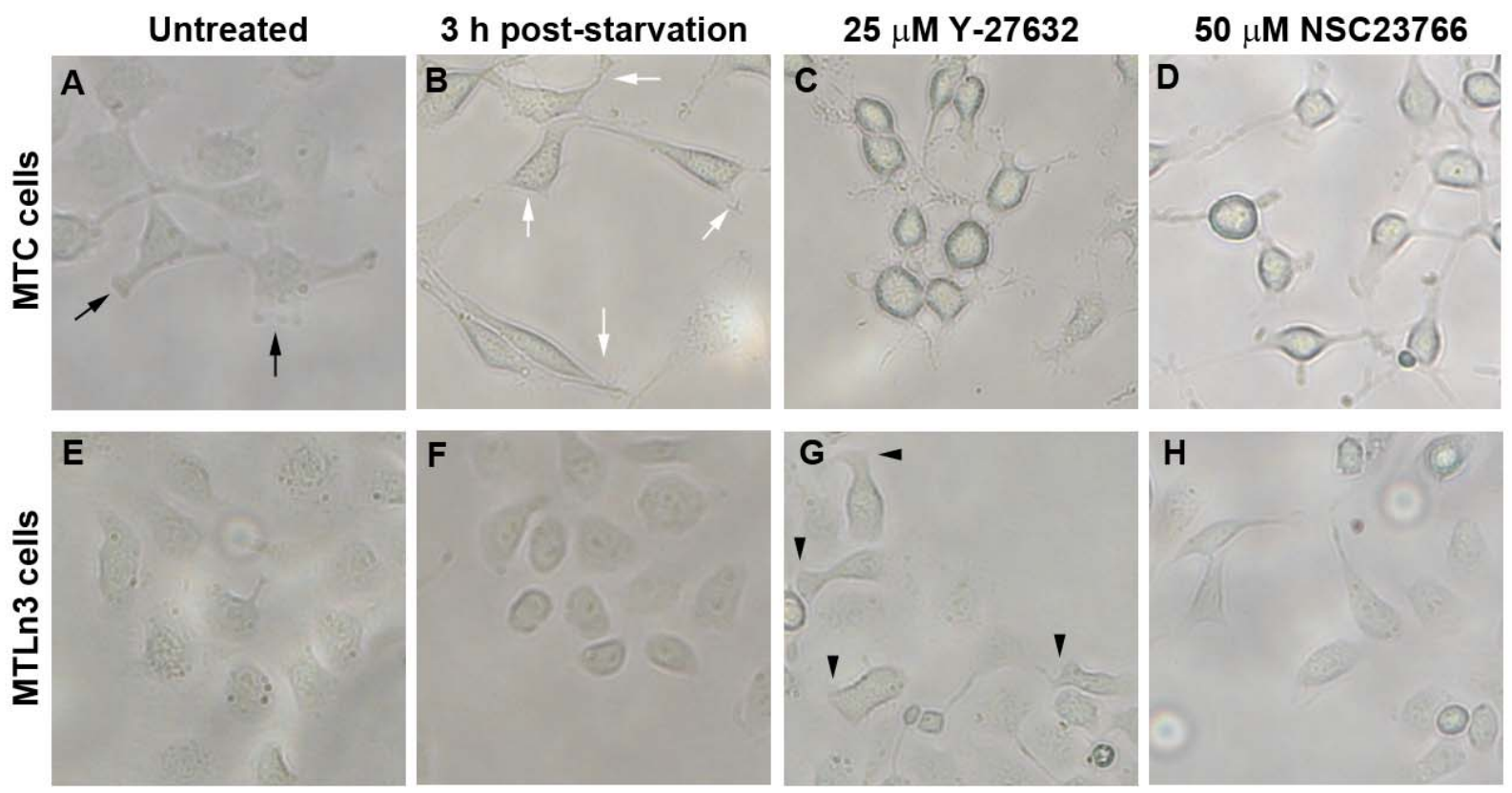

20X magnification

Figure 25. Cellular effects of Rho-GTPase inhibitor treatment on MTC and MTLn3 cells. A) Untreated MTC cells maintained a limited leading edge (black arrows). B) Following a $3 \mathrm{hr}$ starvation, MTC cells lost their leading edge protrusions (white arrows). MTC cells treated with C) $25 \mu \mathrm{M}$ Y-27632 to inhibit ROCK or D) $50 \mu \mathrm{M}$ NSC23766 to inhibit Rac1 exhibited a rounded phenotype. E) Untreated MTLn3 lamellipodial protrusions were difficult to visualize by phase contrast microscopy. F) Starved MTLn3 cells did not exhibit significant changes in cell morphology. G) Treatment of MTLn3 cells with $25 \mu \mathrm{M}$ Y-27632 resulted in lamellipodial protrusions (arrowheads) that resembled untreated MTC lamellipodial protrusions (refer to A, black arrows). H) $50 \mu \mathrm{M} \mathrm{NSC23766} \mathrm{inhibitor} \mathrm{treatment} \mathrm{of} \mathrm{MTLn3} \mathrm{cells} \mathrm{resulted} \mathrm{in} \mathrm{a} \mathrm{mixed}$ cellular phenotype with some cells containing normal lamellipodial protrusions, while some had decreased protrusions. Images were taken at 20X magnification. Results are representative of three to four independent experiments. 

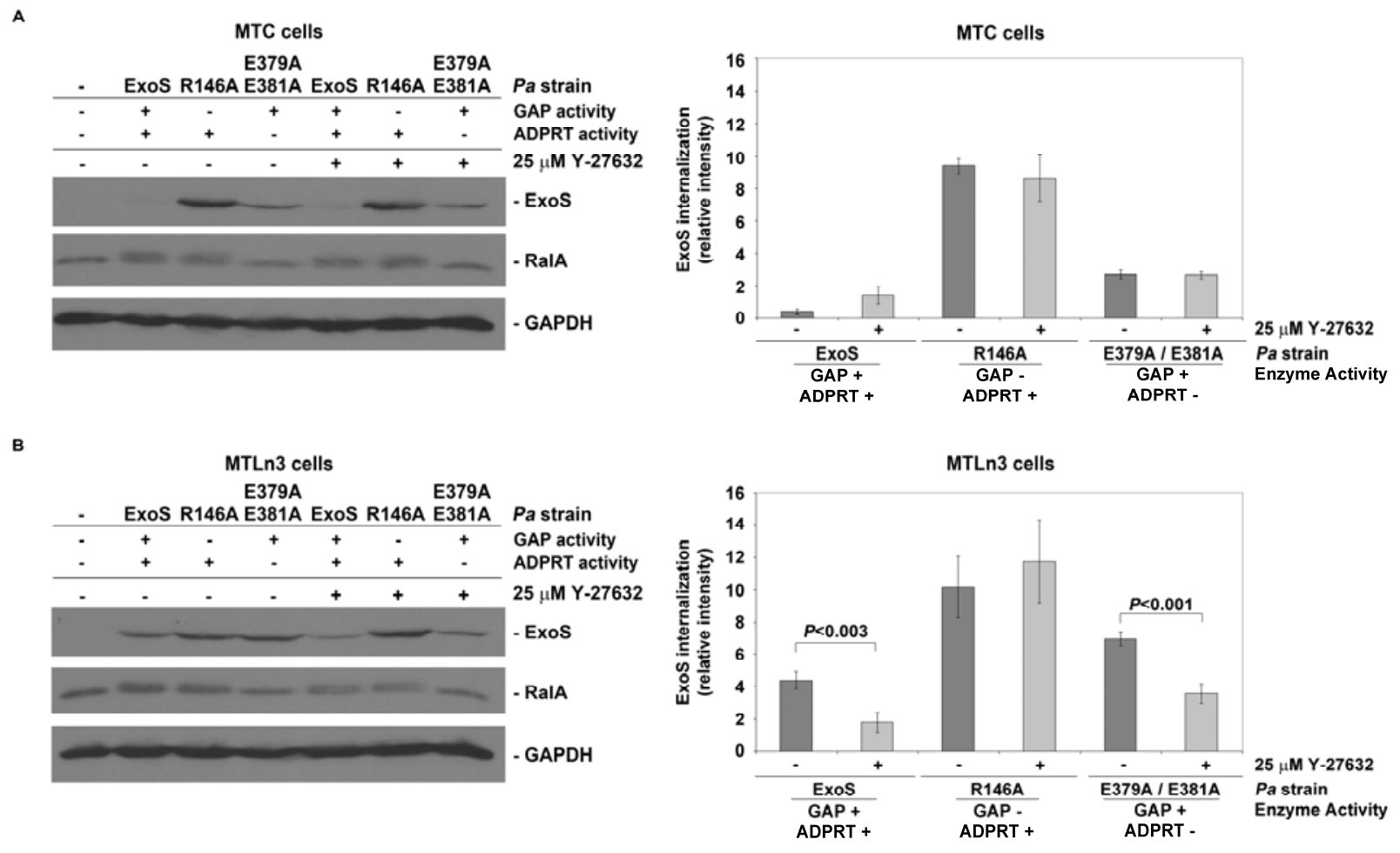

Figure 26. Inhibition of ROCK in MTLn3 cells decreased Pa-T3S sensitivity. A) MTC and B) MTLn3 cells were starved for $3 \mathrm{hr}$ prior to the addition of $25 \mu \mathrm{M}$ Y-27632 for $30 \mathrm{~min}$. Both cell lines treated with or without inhibitor were co-cultured with Pa expressing WT ExoS, an ExoS GAP- (R146A) mutant, or an ExoS ADPRT- (E379A/E381A) mutant for $3.5 \mathrm{hr}$. Left panel, cells were harvested and whole cell lysates were evaluated by immunoblot for ExoS translocation and ADP-ribosylation of RalA. GAPDH served as a protein loading control. Right panel, ExoS translocation into both cell lines was quantified by densitometry and is reported as the mean relative intensity. ExoS translocation into MTLn3 cells was significantly decreased in cells treated with Y-27632 by $P a$ expressing WT ExoS $(P<0.003)$ or the ExoS ADPRT- mutant $(P<0.001)$. Results are representative of three independent experiments. 

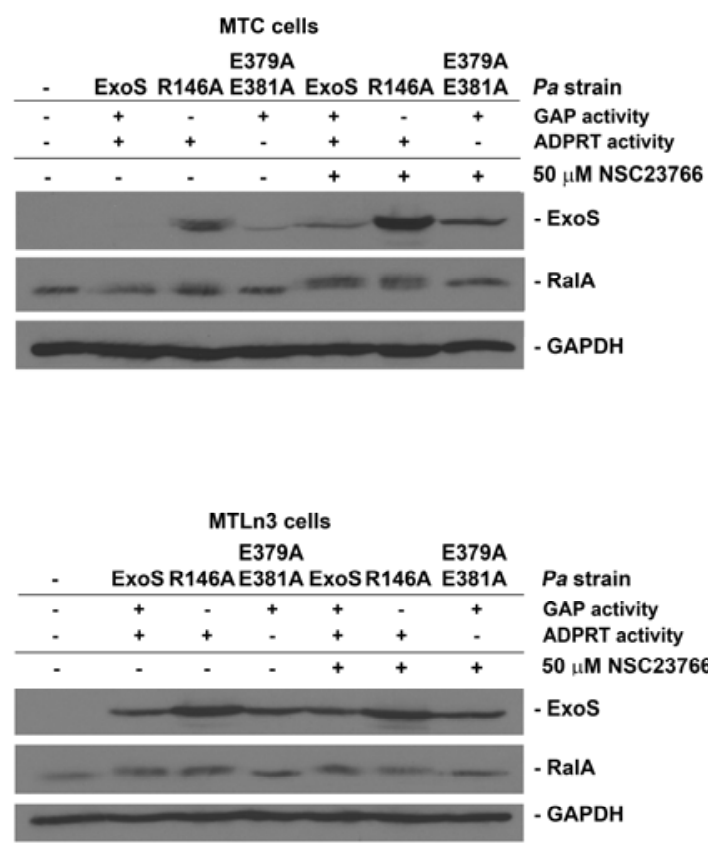
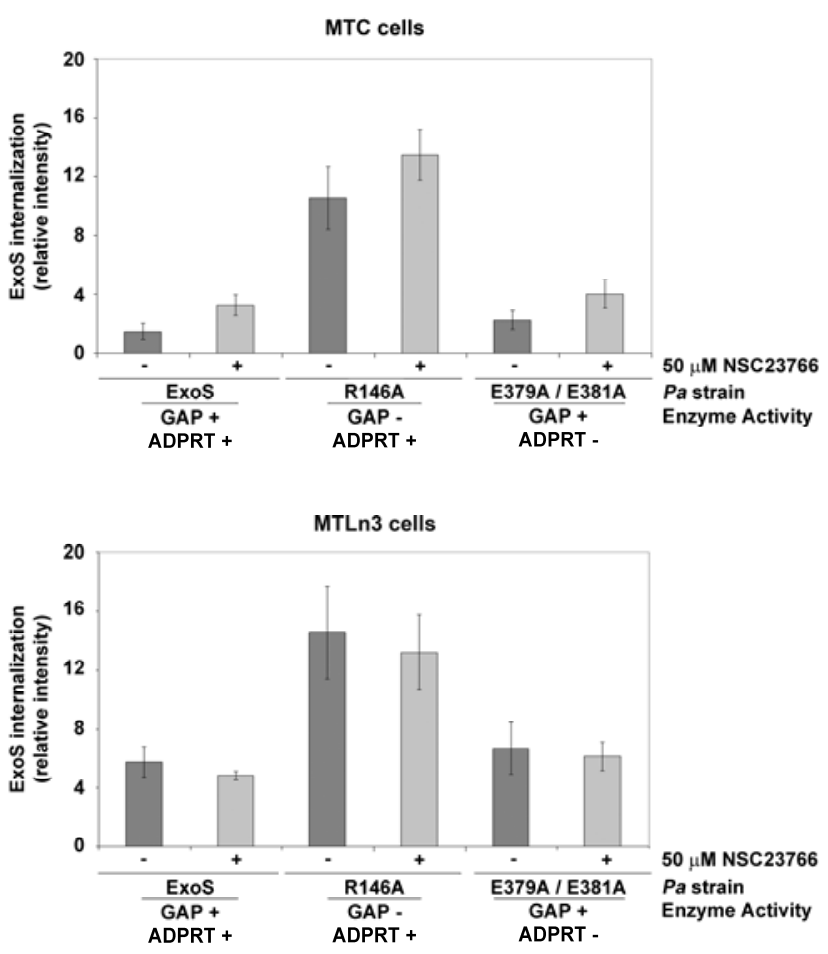

Figure 27. Inhibition of Rac1 in MTC cells increased Pa-T3S sensitivity. A) MTC and B) MTLn3 cells were starved and treated with $50 \mu \mathrm{M}$ NSC23766 for 12-16 hr. Both cell lines treated with or without inhibitor were co-cultured with $\mathrm{Pa}$ expressing WT ExoS, an ExoS GAP(R146A) mutant, or an ExoS ADPRT- (E379A/E381A) mutant for $3.5 \mathrm{hr}$. Cells were harvested and evaluated as described for Figure 26. ExoS translocation into MTC cells was increased with inhibition of Rac1 by all strains; however, increased translocation did not reach statistical significance. Results are representative of four independent experiments. 
Characterizing MDCK cell migration in Pa-T3S. In addition to MDCK monolayers being used in numerous studies to understand wound healing, they have also been widely used as a model system to study $P a$ infection and internalization by polarized epithelial layers $(16,38,87,113$, $131,155,156,158,159,185)$. Current studies support the model that $P a$ can be internalized into incompletely polarized MDCK monolayers in a Rho, PI3K, Akt, and Lyn dependent manner $(155,156,158,159,185)$. Pa internalization can also occur in a Rho independent manner upon disruption of epithelial tight junctions (156). While it is known that wounded epithelial monolayers and disruption of epithelial cell polarity are predisposing factors for susceptibility to $P a$ infection, it remains unknown how Pa-T3S is involved in this process $(62,235,242,245$, 251).

To understand how cell migration during wound healing is involved in susceptibility to Pa-T3S infection, three day confluent monolayers were wounded and allowed to heal for $0-14 \mathrm{hr}$ prior to co-culture with $\mathrm{Pa}$. Due to low levels of ExoS translocation by $\mathrm{Pa}$ expressing WT ExoS (Figs. 21B and 22B), monolayers were treated for $4 \mathrm{hr}$ with $\mathrm{Pa}$ expressing an ExoS GAP-/ADPRTstrain (Fig. 22B) to allow detection of ExoS by IF microscopy. In non-wounded monolayers, minimal Pa binding was observed and as a result, there was no detection of ExoS translocation (Fig. 28A). When Pa was added to cells immediately following wounding (Fig. 28B), low levels of $\mathrm{Pa}$ were found to adhere to and be internalized into cells migrating to close the wound. However, there were low to no detectable levels of translocated ExoS along the entire length of the wound (Fig. 28B, ExoS panel). Internalized $\mathrm{Pa}$ were found to align with actin stress fibers in migrating MDCK cells (Figs. 28B, enlarged image, and 29, all time-points). When wounded monolayers were allowed to heal for $2 \mathrm{hr}$ prior to the addition of bacteria, both the number of $\mathrm{Pa}$ binding to and being internalized along the wound increased (Fig. 28C). ExoS translocation was now detectable in a few locations along the length of the wound. Pa binding, internalization, and ExoS translocation into migrating cells along the wound was most efficient when the bacteria 
were added 4 or $6 \mathrm{hr}$ post-wounding (Figs. 28D and E, respectively). At both of these timepoints, large amounts of bacteria were found associating with the cells. Sites of ExoS induction along the length of the wound were also greater at these time-points. The level of ExoS translocation varied in that some cells were observed to have low levels of ExoS, while others had increased levels of ExoS staining. (Figs. 28D and E; ExoS panels). Interestingly, when $\mathrm{Pa}$ were added $12 \mathrm{hr}$ post-wounding, a large amount of $\mathrm{Pa}$ were able to adhere to and be internalized by migrating MDCK cells, but ExoS translocation was decreased compared to the 4 and $6 \mathrm{hr}$ time-points (Fig. 28F). By $14 \mathrm{hr}$ post-wounding, $\mathrm{Pa}$ binding, internalization, and ExoS translocation into MDCK cells was reduced (Fig. 28G).

In addition to $\mathrm{Pa}$ aligning with actin (Fig. 29, yellow arrows), ExoS was also found to align along actin filaments in migrating MDCK cells (Fig. 29; blue arrows). Consistent findings in this study were: (1) the ability of $\mathrm{Pa}$ to align along actin filaments regardless of wound healing time; (2) $\mathrm{Pa}$ targeting and translocating ExoS into cells along the healing wound; and (3) resistance of the remaining confluent monolayer to $\mathrm{Pa}$ binding, $\mathrm{Pa}$ internalization, and ExoS translocation. The conclusions from these studies were that MDCK cells were susceptible to $\mathrm{Pa}$ internalization immediately following wounding, but were most susceptible to the effects of Pa-T3S 4-6 hr postwounding. 
Figure 28. Length of MDCK wound healing time influenced Pa-T3S sensitivity. Confluent MDCK monolayers were A) unwounded or B-G) wounded and allowed to heal for increasing time (0-14 hr) prior to addition of $\mathrm{Pa}$ expressing an ExoS GAP-/ADPRT- (R146A/E379A/E381A) mutant for $4 \mathrm{hr}$. Cells were stained for extracellular $\mathrm{Pa}$ (yellow + pink in merged image), total $\mathrm{Pa}$ (pink), ExoS (green), and actin (white). White arrows indicate position of wound. Results are representative of three independent experiments. 

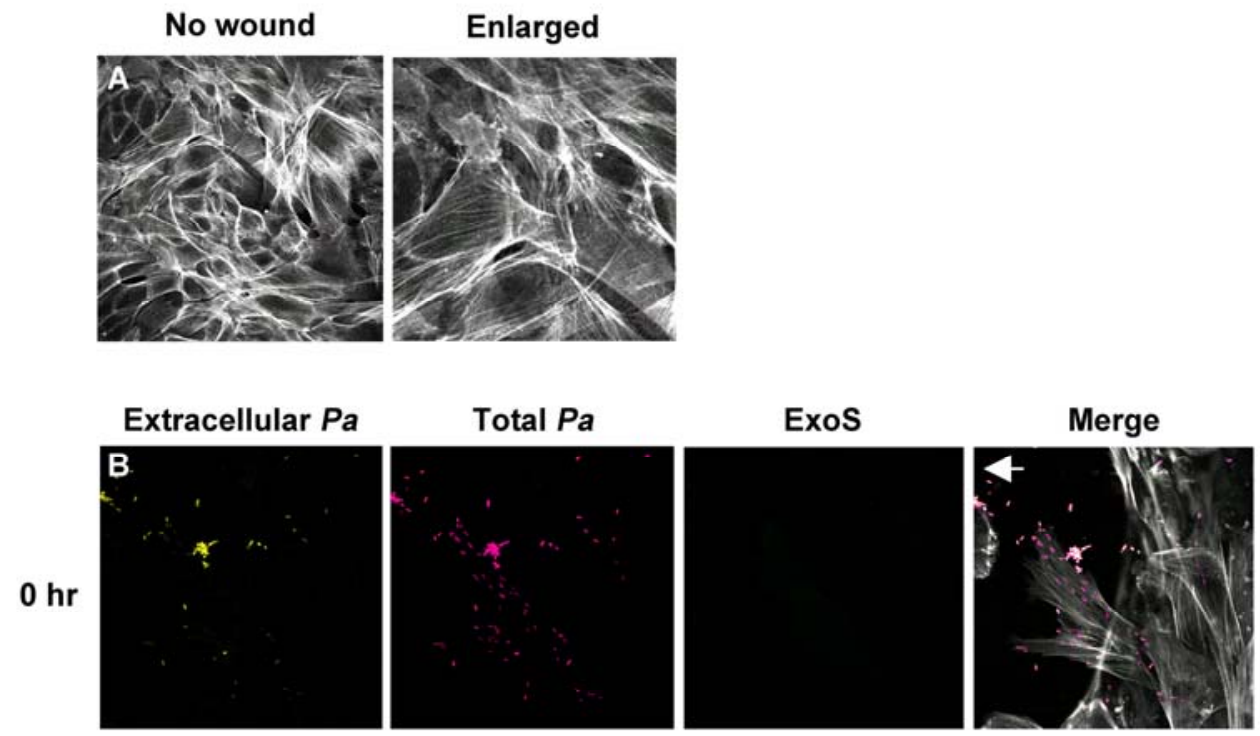

Merge-enlarged
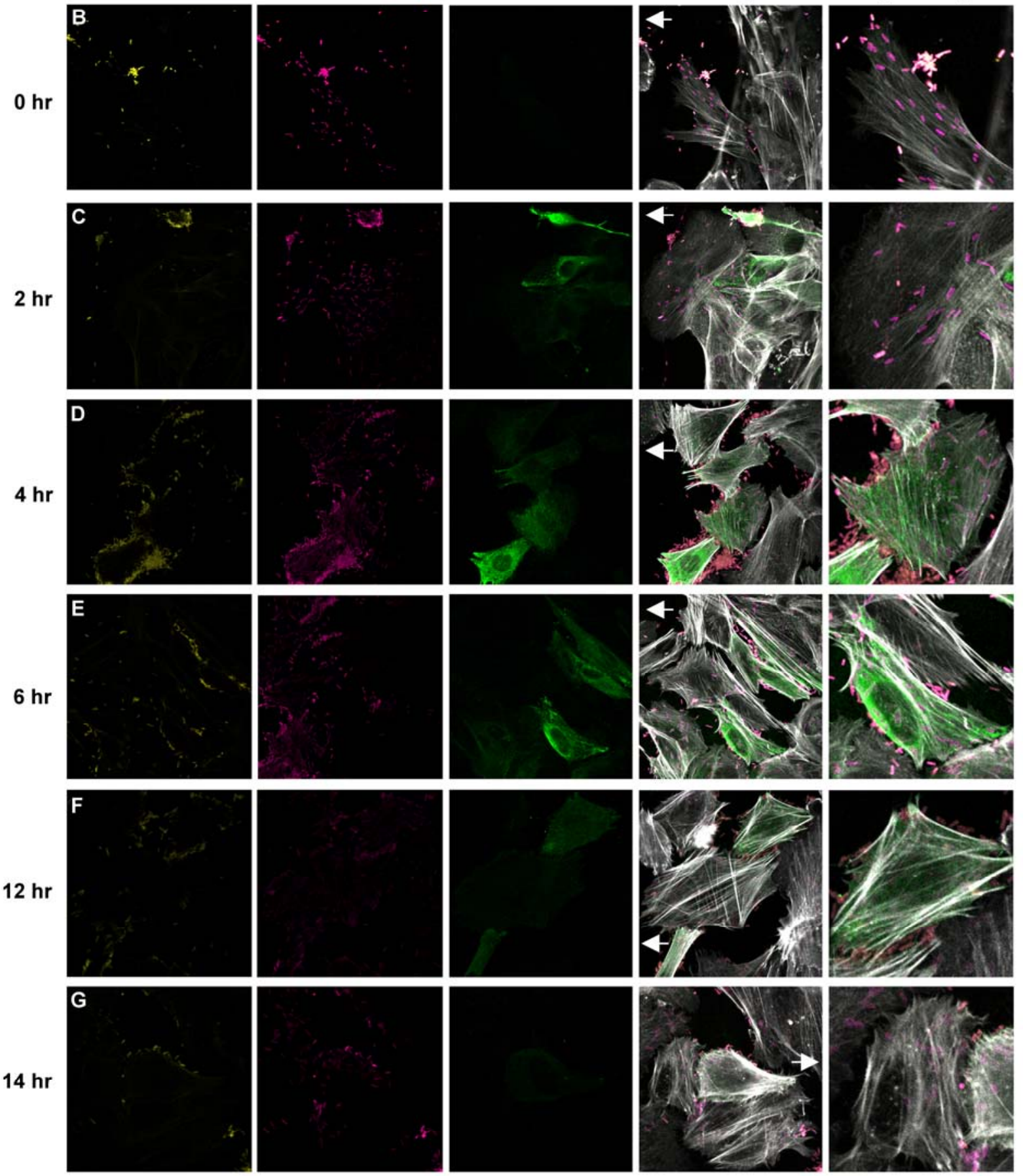
Figure 29. $\mathrm{Pa}$ and ExoS align along actin in migrating MDCK cells. Confluent MDCK monolayers were wounded and allowed to heal for 0-14 hr prior to the addition of Pa expressing an ExoS GAP-/ADPRT- mutant for $4 \mathrm{hr}$. Following co-culture, cells were fixed, blocked, permeabilized, and stained for $\mathrm{Pa}$ (pink), ExoS (green), and actin (white). Bottom panel of each time-point is an enlarged cell from the image above. Yellow arrows indicate Pa aligning with actin. Blue arrows indicate ExoS tracking actin. White arrows indicate direction of wound healing. Scale bars are $20 \mu \mathrm{m}$. Results are representative of three independent experiments. 


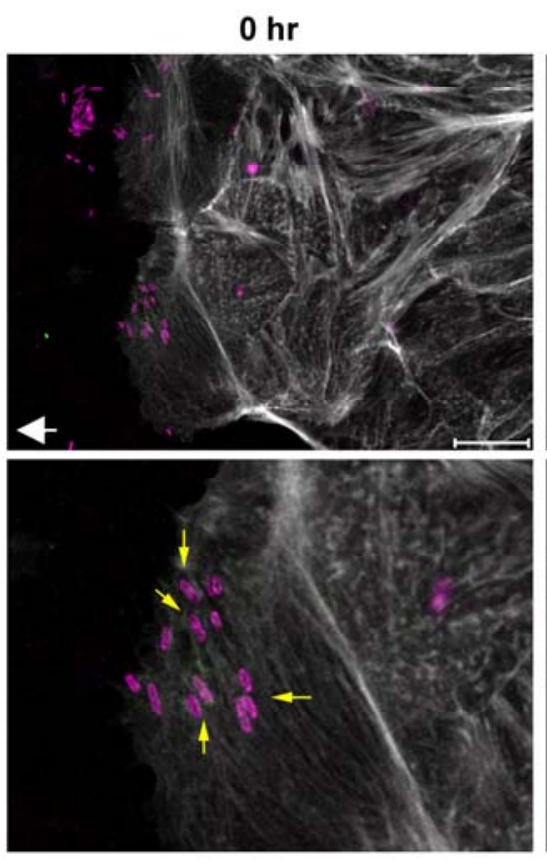

$6 \mathrm{hr}$
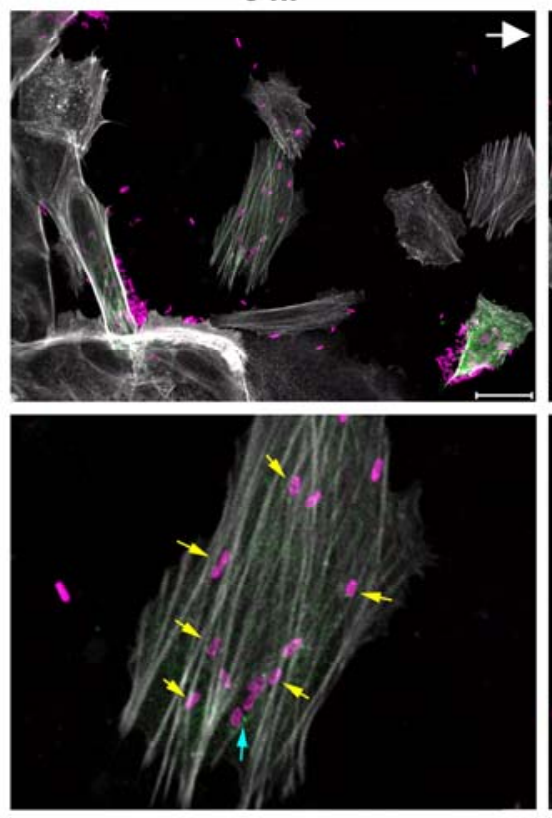

$2 \mathrm{hr}$
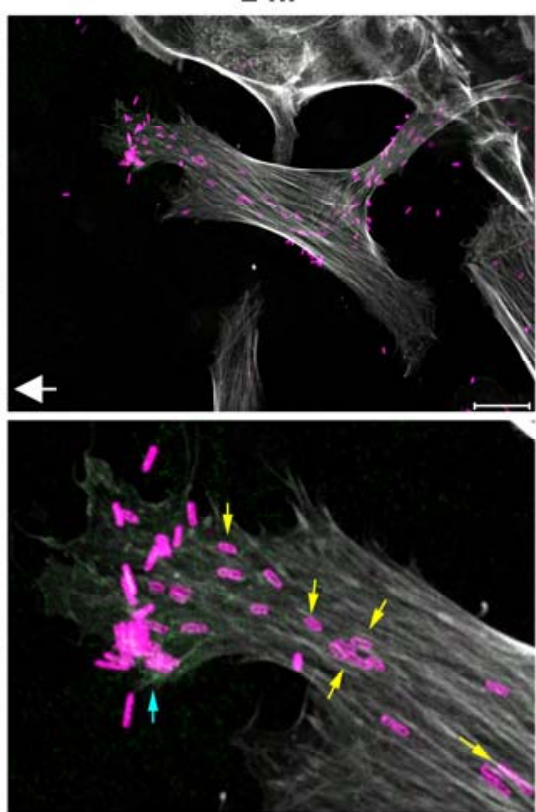

$12 \mathrm{hr}$
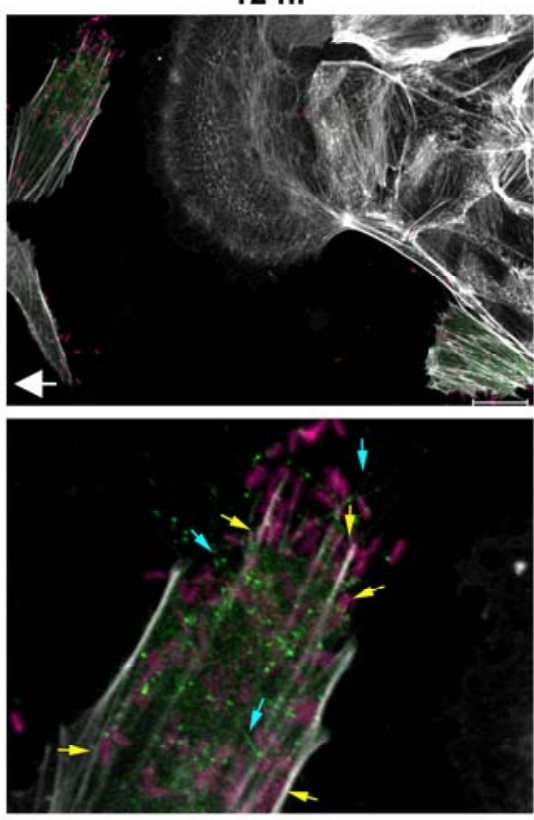

$4 \mathrm{hr}$
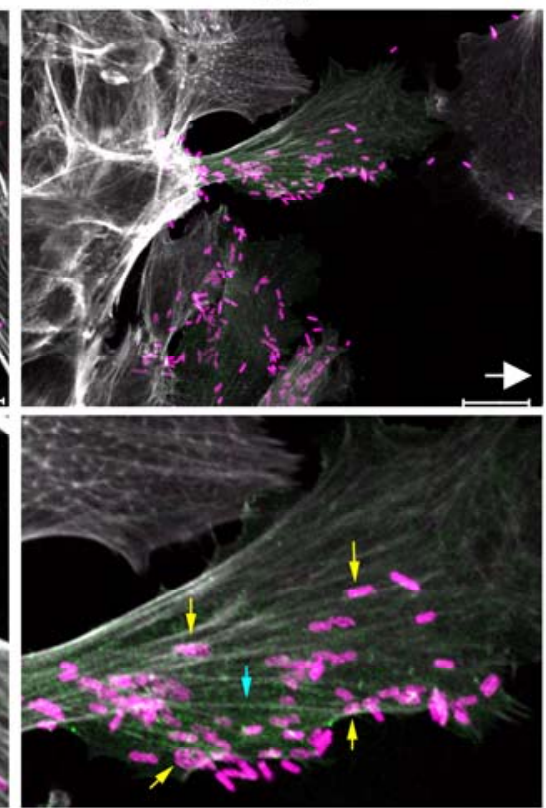

$14 \mathrm{hr}$
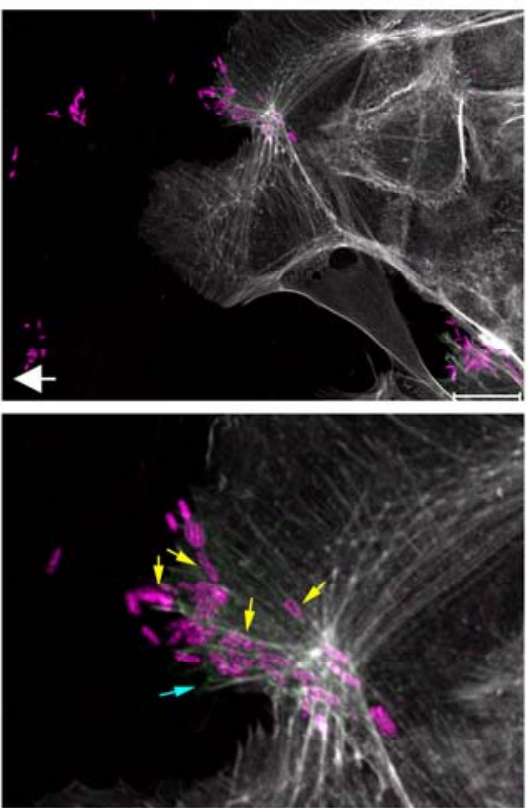
ExoS GAP and ADPRT activities disrupt MDCK wound healing. To determine the effects of ExoS enzyme activity on MDCK wound susceptibility to infection, confluent monolayers were wounded and allowed to heal for $4 \mathrm{hr}$ prior to treatment with Pa expressing WT ExoS, an ExoS GAP- mutant, an ExoS ADPRT- mutant, or an ExoS GAP-/ADPRT- mutant. The $4 \mathrm{hr}$ wound healing time was selected as this was a time-point identified of increased susceptibility to PaT3S (Fig. 28D). When migrating MDCK cells were examined by IF microscopy, there were no substantial differences in ExoS translocation by $P a$ expressing WT ExoS, the ExoS GAPmutant, or the ExoS ADPRT- mutant (Figs. 30A-C). ExoS translocation into wounded cells was only detected with Pa expressing an ExoS GAP-/ADPRT- mutant (Fig. 30D).

Despite ExoS being undetectable in the cell cytosol by IF microscopy with $\mathrm{Pa}$ expressing WT ExoS, an ExoS GAP- mutant, or an ExoS ADPRT- mutant, ExoS translocation into these cells was evident due to observable changes in cell morphology. Pa expressing WT ExoS were found to bind to the leading edge of cells that were migrating to close the wound (Fig. 30A, yellow arrows). The cytotoxic effects of ExoS were detected in these cells by the punctuate nature of the actin cytoskeleton and the loss of actin stress fiber formation (Fig. 30A, top panel). Additionally, the actin cytoskeleton in the lamellipodia of some cells appeared to be contracting which resulted in the formation of long spiked actin structures (Figs. 30A, bottom panel; and 30E, blue arrows). When migrating MDCK cells were treated with Pa expressing an ExoS GAPmutant, lamellipodial contraction was also observed (Fig. 30B). Treatment with an ExoS GAPmutant resulted in the formation of much shorter actin spiked structures as the lamellipodia contracted (Figs. 30B and F, blue arrowheads).

Following treatment with $\mathrm{Pa}$ expressing an ExoS ADPRT- mutant, the lamellipodia where $\mathrm{Pa}$ localized did not contract, however, actin stress fibers within the lamellipodia disappeared (Figs. $30 \mathrm{C}$ and $\mathrm{G})$. The actin which remained in the lamellipodia of cells treated with an ExoS ADPRTmutant resembled adhesions to the ECM (Figs. 30C, top panel, and 30G). It is possible that this 
pattern of actin distribution is due to staining remnants of actin stress fibers which are still attached to focal adhesion complexes after the bulk of stress fibers were broken down by ExoS GAP activity. This phenomenon is best shown in the bottom panel of Figure 30C, where cells further back from the wounded edge (to the right of the blue line) exhibit actin stress fiber formation but those cells where $P a$ are localized (to the left of the blue line) have lost most of their stress fibers. Finally, when migrating MDCK cells were treated with Pa expressing an ExoS GAP-/ADPRT- mutant, actin stress fibers were found in the lamellipodia, and there was no observed contraction of the lamellipodia (Fig. 30D and H).

To determine the mechanism behind the spiked actin structures when MDCK cells were treated with $\mathrm{Pa}$ expressing ADPRT active ExoS (WT ExoS or the ExoS GAP- mutant), levels of phosphorylated ERMs was evaluated. ERMs function to link actin to the plasma membrane at the leading edge $(32,309)$ and are known substrates of ExoS ADPRT activity $(195,196,297)$. Because ExoS ADPRT activity has been shown to target ERM phosphorylation in T24 cells resulting in a wavy actin phenotype (Chapter 3), we wanted to determine if inhibition of ERM phosphorylation could contribute to the actin branching observed in MDCK cells. Through immunoblot analysis, we found that phosphorylation of ERMs was almost completely abrogated when sub-confluent MDCK cells were treated with ExoS ADPRT activity (WT ExoS or ExoS GAP- mutant; Fig. 30I).

To summarize, these results indicate that ExoS GAP activity is responsible for loss of actin stress fiber formation in migrating MDCK lamellipodia, while ExoS ADPRT activity disrupts actin/plasma membrane linkages presumably through disruption of ERM phosphorylation. The increased resistance of MDCK cells to ExoS GAP activity is thought to be due to increased levels of Rho at the leading edge, which allows for increased $\mathrm{Pa}$ internalization. Therefore, in migrating MDCK cells, the combination of both ExoS GAP and ADPRT activities is required for 
contraction of the lamellipodia and loss of stress fiber formation, which prevents cell motility and wound healing. 
Figure 30. ExoS GAP and ADPRT enzyme activities induce independent effects on the MDCK actin cytoskeletal structure during wound healing. Confluent MDCK monolayers were wounded and allowed to heal for $4 \mathrm{hr}$ prior to the addition of $\mathrm{Pa}$ expressing A) WT ExoS, B) an ExoS GAP- mutant (R146A), C) an ExoS ADPRT- mutant (E379A/E381A), or D) an ExoS GAP-/ADPRT- mutant (R146A/E379A/E381A) for $4 \mathrm{hr}$. Cells were fixed and stained for $\mathrm{Pa}$ (pink), ExoS (green), and actin (white). Yellow arrows indicate Pa binding to the leading edge. Blue arrows indicate contraction of the leading edge resulting in long actin filaments $(A)$. Blue arrowheads indicate contraction of the leading edge resulting in short actin microspikes (B). Cells to the left of the blue line show loss of actin stress fibers (C). E-H) Enlargement of panels from A-D showing differences in lamellipodial protrusions. I) Sub-confluent MDCK cells were cocultured with $\mathrm{Pa}$ expressing WT ExoS, an ExoS GAP- mutant (R146A), an ExoS ADPRTmutant (E379A/E381A), or an ExoS GAP-/ADPRT- mutant (R146A/E379A/E381A) for 5 hrs. Cells were fractionated and membrane fractions were assayed by immunoblot to detect phosphorylated ERM (pERM, ezrin-Thr567, radixin-Thr564, and moesin-Thr558). Densitometry analysis showed a decrease in pERM following co-culture with Pa expressing WT ExoS or the ExoS GAP- mutant compared to other strains. Results are representative of one experiment. 

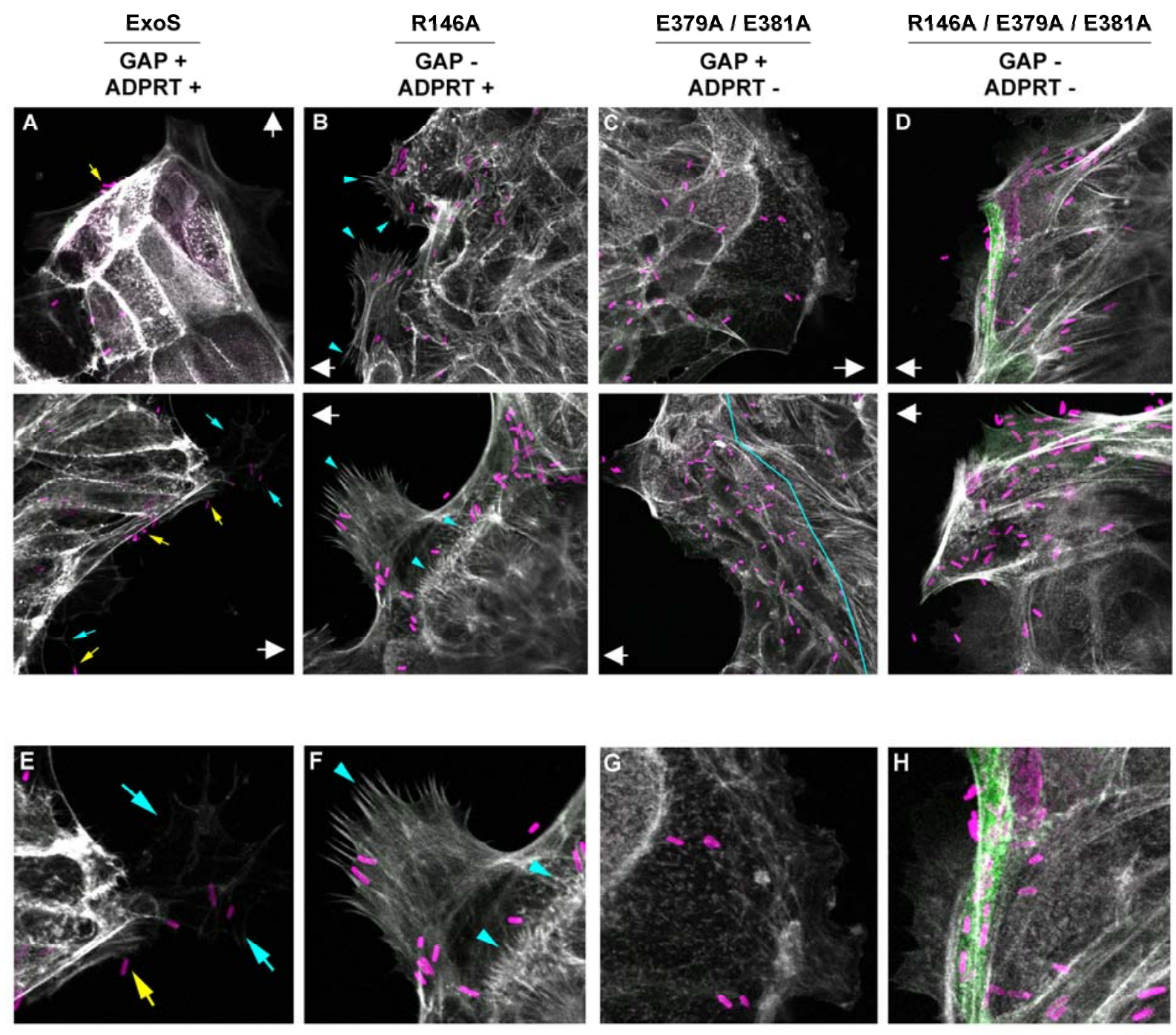

I

R146A

E379A E379A

$\begin{array}{cccccc}- & \text { ExoS } & \text { R146A } & \text { E381A } & \text { E381A } & \text { Pa strain } \\ - & + & - & + & - & \text { GAP activity } \\ - & + & + & - & - & \text { ADPRT activity } \\ - & & & - & & - \text { pERM }\end{array}$




\section{DISCUSSION}

Eukaryotic cell migration is complex process that is regulated by Rho-GTPases (221). In the classical model of migration, Rac1 and Cdc42 regulate leading edge lamellipodial and filopodial protrusions, along with formation of highly dynamic focal complexes $(221,222,277)$. Rho, in concert with ROCK, is responsible for retraction of the rear of the cell body through actomyosin contractions, phosphorylation of myosin light chain, and formation and maintenance of focal adhesions $(12,160,221,222)$. As the cell body moves forward, actin polymerization and contraction must be balanced (reviewed in (225)). Over the last few years it has been discovered that not all cells migrate via this classical pathway. Specifically, Sahai and Marshall (273) showed that protrusions on cells which utilize amoeboid migration are mediated by Rho and ROCK, whereas protrusions on cells which utilize mesenchymal migration require Rac1 and Cdc42. Migrating HT1080 cells, like MTLn3 cells, exhibit activated Cdc42 at the leading edge while Rac1 is located behind the leading edge (150). The reciprocal relationship between Rac1 and Rho dictates which cell migration mechanism dominates $(273,276)$. Importantly, Rhomediated amoeboid cell morphology and metastatic potential have been linked to cancer cell metastasis (273).

The goal of our studies was to understand the relationship between cell migration patterns and the initiation of Pa-T3S infections. We chose three different models of host cell migration patterns for these studies: (1) non-metastatic MTC cells which migrate mesenchymally (217), (2) metastatic MTLn3 cells which migrate by blebbing $(217,225)$, and (3) MDCK cells which can migrate either mesenchymally or collectively (246). Rho-GTPase regulation of the leading edge is also different for each cell type. Specifically, Rac1 is activated at the leading edge in MTC cells, Rho and Cdc42 are activated at the leading edge in MTLn3 cells $(71,72)$, and Rho and Rac1 are activated at the leading edge in non-polarized MDCK cells $(156,173)$. 
In studies utilizing the MTC/MTLn3 cell model system we identified the role of Rho in increased MTLn3 susceptibility to Pa-T3S. ExoS translocation into MTC cells was limited compared to MTLn3 cells, but increased when cells were treated with $\mathrm{Pa}$ expressing ExoS with mutations abolishing GAP activity. Through IF microscopy studies, we identified that increased ExoS translocation into MTLn3 cells was attributed to increased internalization of $P a$ by this cell line, and once Pa were internalized could continue ExoS translocation. Rodent cell lines have been shown to be more sensitive to ExoS GAP activity $(264,270)$ and that ExoS GAP activity towards Rac1 results in a loss of lamellipodium, while down-regulation of Rho results in the production of actin stress fibers $(116,136,167,233)$. Also through IF studies, we observed differences in cell morphology due to ExoS GAP activity. When MTC cells were treated with a GAP active strain (WT ExoS or the ExoS ADPRT- mutant) we observed contraction of the lamellipodium into a highly branched actin network, and a decrease in stress fiber formation, consistent with ExoS GAP targeting Rac1 at the leading edge. In comparison, when MTLn3 cells were treated with an ExoS GAP active strain, limited branched actin morphology was observed at the early time-point that did not become as extensive at the later time-point as was observed in MTC cells. A loss in stress fiber formation was also noted in MTLn3 cells when treated with active ExoS GAP. Our results in MTLn3 cells are consistent with reports that increased Rho in MTLn3 cells suppresses Rac1 at the leading edge and therefore the target of ExoS GAP activity is not accessible, resulting in less drastic cell morphology (72). Based on these observations, we hypothesize that the balance between Rho-GTPase regulation of leading edge dynamics was causing this effect and as a result, MTLn3 cells were resistant to ExoS GAP activity. Furthermore, ExoS ADPRT activity was thought to disrupt phosphorylation of ERMs in MTLn3 cells, which resulted in actin stress fibers protruding past the lamellipodium as the membrane contracted; however, this did not appear to influence host cell susceptibility to Pa-T3S. 
The ROCK inhibitor Y-27632 has been previously used to switch MTLn3 lamellipodial protrusion from a Cdc42-dependent process to a Rac1-dependent process by down-regulating Rho (72). If Rho or Rac1-mediated cell migration patterns influenced T3S, we thought that inhibition of Rho pathways in MTLn3 cells using the ROCK inhibitor would decrease MTLn3 sensitivity to Pa-T3S comparable to that of MTC cells. In support of this hypothesis, we found that by using the ROCK inhibitor in MTLn3 cells, sensitivity of the cells to ExoS GAP activity was decreased. We believe that ROCK treatment of MTLn3 cells prevented $\mathrm{Pa}$ internalization and continued ExoS translocation, which can be confirmed with additional studies. By down-regulating Rac1 in MTC cells we anticipated the observation of a translocation pattern similar to MTLn3 cells. When MTC cells were treated with the Rac1 inhibitor NSC23766, an increase in effector translocation was observed, however the increase was not significant compared to untreated cells.

Together, these results suggest that limited ExoS translocation in MTC cells is due to ExoS targeting Rac1 at the leading edge, resulting in membrane contraction and regulation of Pa-T3S by preventing further ExoS translocation into the cell. Due to decreased levels of Rac1 in MTLn3 cells, the lamellipodia and actin stress fibers remain intact when treated with active ExoS GAP activity. This allows $P a$ to be internalized and continue ExoS translocation. Through these studies, we were able to determine the mechanism behind host cell sensitivity to Pa-T3S by using a clonal cell model system where differences between the cell lines have been characterized. Additionally, these studies provided the first line of evidence that Rho localization to the leading edge of migrating cells and host cell migration properties influence sensitivity or resistance to the enzymatic effects of ExoS GAP activity.

MDCK monolayers have been used as a model system to study wound healing and epithelial cell polarization processes in relation to the $\mathrm{Pa}$ infection process $(16,38,87,113,131,155$, 156, 158, 159, 185). As MDCK monolayers polarize, transepithelial resistance increases and apical and basolateral membranes are segregated by the formation of tight junctions (17). 
MDCK cells have the ability to form a partially polarized monolayer within one day of seeding, which is completely polarized by three to five days post-seeding $(17,156)$. Polarization of monolayers can be disrupted either by wounding (138) or by disruption of tight junctions with EGTA (87). In polarized MDCK monolayers, $\mathrm{Pa}$ is internalized in an actin, Rho, PI3K, Akt, and Lyn dependent manner $(77,155,156,158,159,185)$. In comparison, $P a$ internalization occurs in a Rho independent manner upon disruption of epithelial tight junctions $(87,156)$. Previous studies suggested that disruption of apical-basolateral polarity, either by disruption of tight junctions or wounding, resulted in exposure of receptors on the basolateral membrane where $\mathrm{Pa}$ bound and was internalized (38). As cells polarize, Rho-dependent uptake of $\mathrm{Pa}$ becomes down-regulated as total activated Rho decreases $(155,156)$. Once a monolayer becomes polarized, aggregates of $\mathrm{Pa}$ can bind to the apical membrane and facilitate internalization by activating Lyn tyrosine kinase that, in turn, up-regulates PI3K and Akt, recruiting PIP3 and actin to the apical membrane $(158,159,185)$. Through this mechanism, Pa essentially converts the polarized apical membrane into a basolateral membrane thus facilitating internalization.

Wounded epithelial layers and disruption of epithelial cell polarity are known to be predisposing factors for susceptibility to $\mathrm{Pa}$ infection, but how $\mathrm{Pa}$-T3S was involved in this process remained unknown $(62,87,155,156,235,242,245,251)$. Of the limited studies investigating the role of $\mathrm{Pa}$ effectors with MDCK monolayers, $\mathrm{Pa}$ expressing ExoU was found to induce rapid host cell death and damage when added to polarized apical membranes and extended the area of infection to surrounding cells $(16,87)$. Pa effectors ExoT and ExoS were both able to disrupt $\mathrm{Pa}$ internalization by MDCK, HeLa, and corneal epithelial cells through their Rho-GAP activity (52, 108).

To understand the role of $\mathrm{Pa}$-T3S in wound healing, confluent MDCK monolayers were wounded, allowed to heal for various times, and treated with a $P a$ strain expressing GAPIADPRT- ExoS to optimize detection of ExoS translocation. Pa were efficiently internalized into 
MDCK cells somewhat independent of wound healing time, but ExoS translocation was greatest when Pa were added 4-6 hr post-wounding. A possible explanation for the 4-6 hr time-frame of ExoS translocation is the requirement for actin accumulation at the edge of the wound. We have previously proposed that actin polymerization at the leading edge facilitates initial ExoS translocation into host cells and in strains where ExoS enzyme activity was mutated, actin mediated $\mathrm{Pa}$ internalization (Chapter 3). Additionally, we have shown that $\mathrm{Pa}$ expressing WT ExoS preferentially binds to the leading edge of cells where actin polymerization is the greatest ((33), Chapter 3). Elegant studies by Fenteany et al. have shown that at six hours post MDCK monolayer wounding and microinjection of dextran, detergent-resistant actin is incorporated at high concentrations into several rows of cells behind the wounded edge (83). This high level of actin incorporation behind the wound coincides with multiple rows of cells behind the wounded edge extending lamellipodia, frequently under more forward cells, to drive cell migration and wound healing (79). Thick actin bundles then form in a Rho-dependent manner along the margin of the wound edge to help the wound close evenly, while Rac1 mediates lamellipodial formation on the migrating cells (83). Therefore, the observation that $\mathrm{Pa}$ bound to several rows of cells behind the leading edge and were able to translocate ExoS into these cells is consistent with these cells extending lamellipodia and containing high levels of actin polymerization; while cells further back from the wounded edge do not. In further support of the requirement for actin, both $\mathrm{Pa}$ and ExoS were found to track actin within these migrating MDCK cells. ExoS translocation was found to decrease as wound healing progressed past $6 \mathrm{hrs}$, even though $\mathrm{Pa}$ were still internalized at high numbers up to $14 \mathrm{hrs}$ post-wounding. It is possible that Rhomediated membrane ruffling on migrating cells at the later time-points (12-14 hr) facilitated $\mathrm{Pa}$ internalization but the absence of high actin accumulation associated with the 4-6 hr time-points resulted in less efficient translocation (173). Additionally, the decrease in internalization and translocation at $14 \mathrm{hr}$ could be due to cells reestablishing polarity as the wounds began to close. 
When influences of ExoS enzyme activity on translocation were evaluated, mutations abolishing both ExoS GAP and ADPRT activities were required to induce a significant increase in ExoS translocation. In MDCK cells, actin stress fiber formation is Rho-mediated and lamellipodial formation is Rac1-mediated (83). Since lamellipodia, but not actin stress fibers, were present in MDCK cells treated with active ExoS GAP (WT ExoS or the ExoS ADPRT- mutant), our results suggest that ExoS GAP activity is preferentially targeting Rho in MDCK cells not Rac1. In this regard, if Rac1 was also targeted by ExoS GAP activity, we would have expected to see a decrease in lamellipodial formation in addition to the loss of stress fiber formation. Our results differ slightly from studies showing that down-regulation of Rho by ExoS GAP results in an increase in stress fiber formation $(116,136,167,233)$. A potential reason for this difference is that kinetic analysis showed ExoS GAP activity towards Rho is less than Rac1 (186). If MDCK cells are innately more resistant to ExoS GAP activity and the target of ExoS GAP activity is Rho, then potentially more ExoS would be required to down-regulate Rho in this cell line.

In contrast to T24 cells, MDCK treatment with ExoS ADPRT- mutants did not result in an increase in T3S effector translocation (Chapter 3). However, similar to T24 cells, treatment of MDCK cells with active ExoS ADPRT activity (WT ExoS or the ExoS GAP- mutant) resulted in decreased phosphorylation of ERMs, contraction of the lamellipodia, and formation of actin projections (Chapter 3). When MDCK cells were treated with active ExoS ADPRT activity, the actin projections appeared to remain adherent to the ECM. These results are in contrast to results in T24 cells, where the disruption of actin-membrane linkage by ExoS ADPRT activity resulted in a wavy actin phenotype (Chapter 3 ). It is possible that in MDCK cells, pERMmediated actin membrane linkage is not affected by ExoS ADPRT activity as it is in T24 cells, supporting the notion that the cell line properties of MDCK cells is influencing the effects of T3S.

In MDCK cells, attachment to the ECM by peripheral actin and focal adhesion complexes is mediated by MLC kinase (MLCK), while larger focal adhesions are mediated by RhoA (247). 
Consistent with the presence of focal adhesion complexes regulated by activated MLCK, the lamellipodia and actin projections of MDCK cells treated with Pa expressing WT ExoS or the ExoS ADPRT- mutant appeared to remain adherent to the ECM, as demonstrated by punctate structures within the lamellipodium. In contrast to HT-29 and T24 cells, where focal adhesion complexes are Rho-mediated and are disrupted by ExoS GAP activity, results in MDCK cells indicate that adhesion of actin to the ECM by focal adhesion complexes is not disrupted by ExoS because MLCK is not an identified target of ExoS GAP activity. Taken together, these results imply that both activities must work together to regulate translocation and manipulate the host cell. This is unlike MTC and MTLn3 cells where ExoS GAP activity is predominant or in T24 cells where ExoS ADPRT activity is predominant in T3S regulation and again support the idea the host cell properties are influencing the effects of T3S.

In immunoblot studies of sub-confluent MDCK cells, we found that T3S sensitivity was induced after a $5 \mathrm{hr}$ co-culture period, a time-point later than that observed in other T3S sensitive cells, such as T24 cells (3.5-4 hrs; Chapter 3 ). These results implied that sub-confluent MDCK cells were more resistant to the effects of ExoS than other sensitive cell types. These observations are consistent with the sensitivity levels to Pa-T3S in healing wounds. It is possible that the increased resistance of sub-confluent MDCK cells is the same phenomenon observed in cells that migrated for greater than $6 \mathrm{hr}$. In this regard, sub-confluent cells were seeded and treated with $\mathrm{Pa} 48 \mathrm{hrs}$ later. At this time, it is possible that $\mathrm{Pa}$ are being internalized due to increased Rho-mediated membrane ruffling in the lamellipodium but that ExoS translocation is not very efficient if high levels of actin polymerization is not occurring. While the MDCK cells were subconfluent in this set of studies, they formed micro-colonies which could have acquired polarization over the $48 \mathrm{hr}$ time-frame. Since it is known that as MDCK cells polarize, Rho decreases and Cdc42 increases (156), it is possible that levels of Rho within these micro- 
colonies were decreased and thus Pa-T3S sensitivity was not greater. If cells were treated 4-6 hrs post-seeding Pa-T3S sensitivity might potentially increase.

Collectively, a model that emerges for the role ExoS and Pa-T3S in MDCK wound healing is that (1) following monolayer wounding, multiple rows of cells behind the wound form lamellipodia and begin to migrate to close the wound. (2) Disruption of monolayer polarity exposes basolateral proteins which allows for almost immediate $\mathrm{Pa}$ binding to the leading edge. (3) Pa then forms aggregates on the cell surface activating the Lyn, PI3K, Akt, PIP3 pathway leading to $\mathrm{Pa}$ internalization via an actin and Rho-dependent mechanism. (4) As the wound healing time increases to 4-6 hrs, levels of actin accumulation in multiple rows of cells behind the wound also increases, thereby allowing for T3S translocation of ExoS into MDCK cells by both extracellular and intracellular Pa. (5) T3S is subsequently down-regulated by ExoS translocation through a combination of ExoS GAP and ADPRT activity, which disrupt actin stress fiber formation and phosphorylated ERM-mediated actin linkage to the membrane, respectively. (6) The disruption in actin stress fiber formation along with induction of cell rounding and cell death by ExoS prevents cell migration and wound healing. It is possible that during infection as the cells along the edge of the wounded epithelial layer die and more cells form lamellipodia, this process can continue and lead to dissemination of bacteria to other locations of the body causing bacteremia and septic shock. Another alternative is that as the wounded area becomes larger due to the death of migrating cells this allows for secondary infection by other pathogens.

Through studies of three cell lines with known differences in cell migration patterns, and in combination with previous studies of T24 cells (Chapter 3), the pattern that emerges is that while the effect of ExoS enzyme activity is different in each cell type, increased host cell sensitivity to translocation and $\mathrm{Pa}$ internalization is attributed to increased levels of Rho at the leading edge as compared to the trailing edge. This displacement of Rho renders cells more 
resistant to ExoS GAP activity leading to $P a$ internalization and continued effector translocation within the host cell. 


\section{ACKNOWLEDGEMENTS:}

Strain PA103 UUT was provided by Dara Frank (Medical College of Wisconsin, Milwaukee, WI) and ExoS-HA was provided by Joseph Barbieri (Medical College of Wisconsin, Milwaukee, WI). MTC cells were provided by John S. Condeelis (Albert Einstein College of Medicine, Bronx, NY) and MTLn3 cells were provided by Scott Weed (West Virginia University Mary Babb Cancer Center). We would like to acknowledge the assistance of Amanda Ammner in MTLn3 cell culturing conditions. Imaging experiments and image analysis were performed in the West Virginia University Imaging Facility with the assistance of Karen Martin. Statistical analyses were performed by Gerry Hobbs (West Virginia University Department of Statistics). 
Chapter 5: Discussion 
The opportunistic pathogen Pseudomonas aeruginosa $(\mathrm{Pa}$ ) can cause infections with severe morbidity and mortality in any tissue or cell type. Mortality rates for $\mathrm{Pa}$ bloodstream and nosocomial infections can range from $33-60 \%(189,253-255,325,335) . P a$ is the leading cause of death in patients with cystic fibrosis, and it is known to cause approximately $35 \%$ of recurrent urinary tract infections and up to two-thirds of contact lens related corneal infections $(8,43,164$, 239, 266). Pa uses numerous virulence factors that are cell-associated or secreted by one of five secretion systems during its infectious process. However, the host cellular alteration that allows for the establishment of infection in so many tissue and cell types is unknown. Understanding how $\mathrm{Pa}$ infections are established is important for the development of novel antiPseudomonal therapies due to increased resistance of $\mathrm{Pa}$ to antibiotics and the ability of bacteria to adapt to their surroundings.

One $\mathrm{Pa}$ virulence factor implicated in the establishment of $\mathrm{Pa}$ infection is the type III secretion (T3S) system (reviewed in (129)). The T3S system is a nano-macromolecular structure that spans both bacterial membranes and is used by numerous Gram-negative bacteria to establish infection (reviewed in (51)). Once the Pa-T3S needle comes in contact with the host cell, the translocon proteins, PopB and PopD, are secreted, and in conjunction with the needle tip protein, PcrV, form a translocon channel in the eukaryotic membrane $(119,283)$. Pa then modulates host cell properties through the secretion of four effector proteins, ExoS, ExoT, ExoU, and ExoY. By providing a conduit for the direct translocation of effector proteins from the bacterial cytosol into the host cell cytosol, the T3S system allows $P a$ to bypass the host immune response to establish an infection. The importance of T3S and ExoS in the establishment of infections is documented by the increased disease morbidity and mortality of infections caused by $\mathrm{Pa}$ isolates expressing the T3S system as compared to $\mathrm{Pa}$ isolates not expressing the T3S system $(73,130,269,296)$. While the expression of the needle-tip protein PcrV alone can induce an increase in disease severity, co-expression of PcrV with the effectors ExoU and ExoS 
have been shown to further increase disease morbidity and morality $(130,269,296)$. Together, these results show the importance in understanding how the T3S system contributes to the establishment of infection.

$P a$ is known to target wounded epithelial barriers when establishing infection $(62,235,242,245$, 251). Eukaryotic membranes are structured to resist channel and pore formation as occurs during T3S channel formation and our question was what is the cellular compromise that allows the translocon channel to form in the host cell membrane and how does this relate to the initiation of $\mathrm{Pa}$ infection. Previous studies implicated the role of asialo-GM1, the cystic fibrosis transmembrane receptor (CFTR), and epithelial cell polarity in Pa-T3S, but no eukaryotic cell receptor for the $\mathrm{Pa}$-T3S translocation proteins has been identified to date $(62,87,156,243)$. Several studies hypothesized that membrane cholesterol and lipid rafts were involved in Pa-T3S translocation and PopB and PopD membrane insertion, but due to differences in experimental systems, the role of cholesterol in Pa-T3S translocon insertion and function also remains unknown $(80,132,283)$. Previous studies from our laboratory identified that the majority of host cell types were sensitive to Pa-T3S; however, two cell types were found to be resistant to PaT3S polarized epithelial cells and promyelocytic HL-60 cells $(206,271)$. Sensitivity to Pa-T3S could be induced in these two cell lines by disruption of epithelial polarity or by HL-60 cell differentiation with the phorbol ester, 12-O-tetradeconylphorbol-13-acetate (TPA) $(206,271)$. But again, the cellular mechanism underlying Pa-T3S sensitivity was unknown.

Eukaryotic cell sensitivity to Pa-T3S can be assayed by detection of translocated ExoS-HA, ExoS ADP-ribosylation of RalA, or membrane insertion of the translocon protein PopB $(95,96$, 271). The Pa-T3S effector ExoS is known to have GTPase activating protein (GAP) activity that targets Rho family GTPases, and ADP-ribosyltransferase (ADPRT) activity that targets proteins such as the Ras family GTPases, Rabs, cyclophilin A, and the ERM (ezrin, radixin, and moesin) family of proteins $(69,95,96,106,116,135,136,167,195,196,205,233)$. The first study in 
this dissertation, presented in Chapter 2, used ExoS as a tool to identify host cell factors influencing the establishment of $\mathrm{Pa}$ infections, which led to the identification of eukaryotic leading edge and cell migration properties in initiation of $\mathrm{Pa}$ infections. The second study, presented in Chapters 3 and 4, used the T3S modulatory influence of ExoS to further explore the role of eukaryotic cell migration in Pa-T3S.

The first approach in understanding how the host cell is involved in Pa-T3S was to use cell lines known to be sensitive (HT-29 cells) to Pa-T3S (Chapter 2) (33). Through a large series of studies targeting many host cell properties, only methyl- $\beta$-cyclodextrin $(M \beta C D)$ and perfringolysin O (PFO) were able to induce HT-29 cell resistance to Pa-T3S as documented by a decrease in ExoS translocation, a decrease in ExoS ADP-ribosylation of RalA, and/or a decrease in PopB membrane insertion. Both of these agents modulate host cell membranes in a cholesterol-dependent process. In studies investigating the role of eukaryotic cell membrane cholesterol in Pa-T3S we found that neither modulation of cholesterol nor modulation of lipid rafts could inhibit Pa-T3S sensitivity. Instead, loss of HT-29 cell adherence to the tissue culture matrix correlated with inhibition of $\mathrm{Pa}-\mathrm{T} 3 \mathrm{~S}$ (33).

Upon recognition of host cell adherence properties in Pa-T3S sensitivity, we investigated the type of host cell adherence mechanisms to the cell culture matrix that could be involved in T3S. These studies directed our attention to the role of Rac1 and Cdc42 mediated focal complexes at the leading edge in Pa-T3S, while inhibition of focal complexes using ROCK or Src kinase inhibitors did not affect T3S sensitivity (33). Together these studies indicate that eukaryotic cell leading edge properties were potentially involved in Pa-T3S host cell sensitivity. Host cell migration is mediated by cell signaling pathways involving Rac1, Cdc42, Rho, phosphatidylinositol 3-kinase (PI3K), IQ-motif-containing GTPase activating protein 1 (IQGAP1), integrins, actin, and microtubules. Upon investigation of how these proteins were involved in PaT3S, Rac1, Cdc42, and actin were found to function first, followed by microtubules and IQGAP1, 
with PI3K playing a minor role. To relate LE architecture to previous $\mathrm{Pa}$-T3S sensitivity studies, HT-29 cells were monitored by IF microscopy studies under T3S-sensitive and resistant conditions. From these studies, we found that in T3S-sensitive cells, there was a close association between cortical actin with Rac1 or PI3K in the direction of cell migration. Pa was also found to preferentially bind to the leading edge of HT-29 cells under T3S sensitive conditions. Treatment of HT-29 cells with M $\mathrm{MCD}$ and PFO, disrupted leading edge actin colocalization with Rac1 and PI3K, and this coincided with the loss of HT-29 cell sensitivity to PaT3S (33). In addition, after inhibition of T3S by treatment with PFO, $\mathrm{Pa}$ binding became more random and occurred at intercellular junctions rather that at the leading edge of the cell. Together, the role of leading edge focal complexes, the role of proteins involved in cell migration at the leading edge, and the role leading edge architecture in HT-29 cell sensitivity to Pa-T3S led to the hypothesis that eukaryotic cell leading edge properties are an important factor for host cell sensitivity to Pa-T3S (33).

Studies presented in Chapter 2 provided evidence that $\mathrm{Pa}$ was utilizing changes in eukaryotic cell polarity, specifically the properties regulating leading edge migration, in establishing infection. In this regard, we found that the requirement for membrane cholesterol in Pa-T3S, but not modulation of cholesterol or modulation of lipid rafts is consistent with the role of cholesterol at the leading edge of migrating cells. Cholesterol has been proposed to alter plasma membrane viscosity to allow for actin-mediated membrane protrusion during cell migration and that $M \beta C D$ depletion of cholesterol disrupts the polarized distribution of cellular proteins that regulate migration $(193,267)$. The model that we propose from these studies is that leading edge cortical actin polymerization, regulated by activated Rac1 and Cdc42 at the leading edge, is required for insertion of the translocon in the membrane. This model is consistent with studies by Mejia et al. which showed that actin polymerization was also involved in translocon insertion in Yersinia Ysc T3S $(33,207)$. Our model also predicts that since Pa does not engage integrin, 
which induces actin polymerization, $\mathrm{Pa}$ is utilizing host cell leading edge properties that mediate actin polymerization during cell migration for its infectious process (33).

Based on our previous results hypothesizing that the establishment of $P a$ infections is linked to eukaryotic cell migration properties, the second study, which used highly migratory T24 cells as a cell culture model and ExoS as a T3S modulatory tool, allowed dissection of the cellular $\mathrm{Pa}$ infectious process. The use of ExoS to manipulate Pa-T3S is supported by studies by Aili et al. (4), which showed that ExoS expressed by the Yersinia Ysc T3S system had the ability to regulate effector translocation into host cells. From studies described in Chapter $3, \mathrm{~Pa}$ expressing ExoS with mutations that altered ExoS GAP and ADPRT activities was found to enhance ExoS and PopB translocation into T24 cells, thus indicating that ExoS was targeting cellular processes that affected T3S translocation. Consistent with results from the first study (Chapter 2), we again observed that $\mathrm{Pa}$ expressing WT ExoS preferentially localized to the leading edge of migratory cells. However, interestingly and in contrary to our hypothesis that actin-plasma membrane associations at the leading edge facilitated T3S translocation, more efficient T3S translocation by ExoS ADPRT- or GAP-/ADPRT- mutants was associated with $\mathrm{Pa}$ binding within the lamellipodium.

In dissecting the mechanism underlying T3S translocation, we found that $\mathrm{Pa}$ expressing ExoS deficient in GAP activity could be internalized, and that these internalized $\mathrm{Pa}$ could secrete ExoS inside the host cell (Chapter 3). As a result, the binding observed within the lamellipodium of T24 cells by $\mathrm{Pa}$ expressing ExoS with a GAP- mutation, could now be attributed to internalized $\mathrm{Pa}$ localizing within the lamellipodium. Previous studies have reported the antiphagocytic properties of ExoS GAP activity, but this is the first report of ExoS secretion by internalized $\mathrm{Pa}(65,100,263)$. These results are also consistent with recently published studies in Yersinia, whereby internalized Yersinia could continue to translocate low levels of effectors once internalized (354). 
$\mathrm{Pa}$ expressing ExoS with mutations in ADPRT activity were found enhance ExoS translocation into T24 cells by external $\mathrm{Pa}$, which provided evidence that ExoS ADPRT activity was targeting and inactivating a cellular protein(s) required for T3S translocation. ERMs (ezrin, radixin, moesin) are responsible for linking actin to the plasma membrane and are known to be a substrate of ExoS ADPRT activity $(32,195,196,297,309)$. ADP-ribosylation of ERMs by ExoS was previously found to interfere with ERM phosphorylation (pERM) $(195,297)$. Consistent with this notion, ExoS ADPRT activity disrupted actin-plasma membrane anchoring and disruption of pERM/ezrin co-localization at the leading edge in conjunction with interruption of T3S translocation. The mechanism underlying the greater levels of translocation of an ExoS GAPIADPRT- mutant related to the ability of $\mathrm{Pa}$ to become internalized. Interestingly, these internalized $\mathrm{Pa}$ were found to align with actin filaments within T24 cells, an ability that was interrupted by ExoS ADPRT activity. In addition, the inability of Pa expressing an ExoS GAPmutant, which retained active E379 and/or E381 ADPRT residues, to align with actin filaments in T24 cells is consistent with ExoS ADPRT activity disrupting actin-plasma membrane associations required for Pa-actin alignment. Finally, ExoS was also found to align with actin, independent of its enzyme activity, suggesting the importance of actin in ExoS translocation into the host cell (Chapter 3).

Taken together these studies advanced the understanding of the host cell mechanism underlying host cell sensitivity to Pa-T3S and how ExoS regulates T3S translocation. Specifically, $\mathrm{Pa}$ expressing WT ExoS bind to the leading edge of migrating cells where actin linked to the membrane by pERM proteins is accessible to ExoS. Once ExoS is translocated into the host cell, ExoS GAP activity targets Rho-family GTPases to interfere with $\mathrm{Pa}$ internalization, while ExoS ADPRT activity disrupts the actin membrane association. The studies presented here are consistent with studies showing that ExoS GAP activity, like Yersinia YopE, can function to regulate effector translocation (4). An unexpected and novel finding in 
these studies was that ExoS ADPRT activity can also serve to regulate T3S translocation. To date, no studies had been conducted investigating whether ExoS enzyme activity could regulate translocation in the host cell during a $\mathrm{Pa}$ infection, thus highlighting the novel findings of our studies to the understanding of Pa-T3S. Our studies also describe the paradoxical role of ExoS in the $\mathrm{Pa}$ infectious process, whereby the cytotoxic effects of ExoS first contribute to the establishment of $\mathrm{Pa}$ infections. These same cytotoxic effects then interfere with $\mathrm{Pa}$ internalization and T3S translocation to limit the $\mathrm{Pa}$ infectious process and help maintain its extracellular, opportunistic lifestyle.

Realizing the potential for leading edge properties to influence T3S from the studies presented in Chapters 2 and 3, we explored how cells with different migration properties influenced the $P a$ infectious process, again using ExoS to monitor $\mathrm{Pa}$ infections. For these studies we utilized three cell models known to differ in regulation of leading edge dynamics and, as a result, differing cell migration patterns. To investigate potential differences in host cell sensitivity due to differences in host cell migration patterns, T3S sensitivity in non-metastatic MTC cells was compared to its clonal metastatic MTLn3 sub-line. We found that increased MTLn3 Pa-T3S sensitivity was attributed to Pa internalization and continued ExoS translocation within the cell (Chapter 4). Increased MTLn3 sensitivity was also found to be a factor of differences in Rhomediated cell migration properties.

Rac1 is activated at the leading edge in MTC cells, while in MTLn3 cells, Rho and Cdc42 are activated $(71,72)$. The increased activation of Rho in MTLn3 cells has been shown to suppress Rac1 activation (72). Our IF microscopy studies showed that the MTC actin cytoskeleton became highly branched following treatment with $P a$ expressing active ExoS GAP activity. In comparison, MTLn3 cells appeared to be more resistant to ExoS GAP activity in that cell rounding and actin-branching were not as extensive as was observed in the MTC cells. It is likely that the limited change in morphology observed in the MTLn3 cells is due to ExoS GAP- 
mediated down-regulation of Rho. It is known that Rho is a target of ExoS GAP, however, recent studies have shown that the catalytic activity of ExoS GAP towards Rho is less than towards Rac1 and Cdc42 (186). Thus, it is possible that the limited rounding in MTLn3 cells when treated with $\mathrm{Pa}$ expressing ExoS with active GAP reflects low levels of catalytic inactivation of Rho, while the highly branched morphology observed in MTC cells reflects the high catalytic activity of ExoS GAP towards Rac1. Together, these results support the notion that MTC cells are more sensitive to the effects of ExoS GAP activity on the host cell actin cytoskeleton. Through the use of the ROCK inhibitor, Y-27632, and the Rac1 inhibitor, NSC23766, we were able to alter MTC and MTLn3 sensitivity to Pa-T3S. By inhibiting ROCK, MTLn3 sensitivity was reduced to levels comparable to MTC cells, and in turn, inhibition of Rac1 increased Pa-T3S sensitivity in MTC cells, thus supporting the idea that activated Rho is suppressing Rac1 in MTLn3 cells and that this influences MTLn3 cell sensitivity to Pa-T3S.

Together, these results indicate that at the leading edge in MTC cells, ExoS targets Rac1, resulting in membrane contraction and actin cytoskeletal rearrangements that may function in preventing further ExoS translocation into the cell. In MTLn3 cells, the increase in activated Rho suppresses Rac1, and together with the limited catalytic inactivation of Rho by ExoS GAP, MTLn3 cells are resistant to the anti-internalization properties of ExoS GAP activity allowing for $\mathrm{Pa}$ internalization and continued ExoS translocation within the cell.

The third cell model system used in the last set of studies was a Madin-Darby canine kidney (MDCK) wounded monolayer. The purpose of using this model system was to study how host cell properties which control collective migration during wound healing influences Pa-T3S sensitivity. From these studies we found that $\mathrm{Pa}$ expressing WT ExoS bound to the leading edge of cells migrating to close the wound. While Pa internalization occurred in cells that were wounded up to $12 \mathrm{hr}$, ExoS translocation was most efficient when Pa were added 4-6 hours post-wounding. MDCK cells are known to extend lamellipodia in multiple rows of cells behind 
the wounded edge, and at $6 \mathrm{hr}$ post-wounding, an increase in actin polymerization occurs in these cells $(79,83)$. Our observations that $\mathrm{Pa}$ binding to and translocating ExoS into cells both at the immediate wounded edge and to cells several rows behind the wounded edge during this time-frame are consistent with the requirement of actin polymerization in Pa-T3S. Unlike in T24 cells where ADPRT activity predominantly regulated Pa-T3S translocation, and in MTC and MTLn3 cells where ExoS GAP activity predominantly regulated Pa-T3S translocation, MDCK cells required both ExoS GAP and ADPRT activities to regulate translocation. ExoS GAP activity was found to disrupt actin stress fiber formation, while ExoS ADPRT activity disrupted actin linkage to the cell membrane (Chapter 4). Increased $\mathrm{Pa}$ internalization by MDCK cells is believed to be due to increased levels of activated Rho in cells migrating to close the wound. Similar to MTLn3 cells, the increase in Rho would allow for $\mathrm{Pa}$ internalization and continued ExoS translocation. However, since MDCK cells exhibit differences in migration where actin polymerization is greatest $4-6 \mathrm{hrs}$ post-wounding, ExoS translocation is not as great as is observed in MTLn3 cells when treated with Pa expressing WT ExoS.

Together the last set of studies suggest that while the effect of ExoS enzyme activity is different between each of the three cell types, a commonality is that increased host cell sensitivity is attributed to increased levels of Rho at the leading edge. This displacement of Rac1 from the leading edge renders the cells resistant to ExoS GAP activity where $\mathrm{Pa}$ are internalized and continue T3S translocation from within the host cell.

The model that emerges from using ExoS to understand the role of the host cell in Pa-T3S is that $\mathrm{Pa}$ expressing WT ExoS preferentially binds to the leading edge of migrating eukaryotic cells where Rac1 and Cdc42-mediated actin polymerization is the greatest (Fig. 7A). ExoS is then translocated into the host cell in an actin dependent manner. ExoS GAP activity prevents $\mathrm{Pa}$ internalization and continued T3S translocation by mediating the hydrolysis of Rac1-GTP to Rac1-GDP, to prevent actin mediated membrane ruffling. We believe that ExoS ADPRT activity 
functions to limit translocation by targeting the ERM family proteins where inhibition of ERM phosphorylation results in disruption of the actin-membrane linkage required for ExoS translocation. In comparison, migrating cell lines which contain activated Rho at the leading edge are more resistant to the effects of ExoS enzyme activity (Fig. 7B). In this model, T3S translocated ExoS GAP activity has a low affinity for Rho, whereby membrane ruffling would not be inhibited and $\mathrm{Pa}$ could be internalized and continue translocation from within the host cell. While ExoS ADPRT activity appears to disrupt actin-membrane linkages, the presence of either Rac1 or Cdc42 at the leading edge results in maintenance of some actin cytoskeletal network, which allows for continued ExoS translocation.

In conclusion, in contrast to Yersinia, $\mathrm{Pa}$ does not have the ability to induce actin polymerization which results in T3S translocon insertion into the host cell membrane (207). As an opportunist, $\mathrm{Pa}$ appears to preferentially target wounded epithelial barriers where actin polymerization is mediated by the host cell. Pa then utilizes the changes in membrane dynamics to insert the translocon channel, and to translocate effectors into the host cell to interrupt $\mathrm{Pa}$ internalization. As an additional means of supporting survival, $\mathrm{Pa}$ uses ExoS limit T3S translocation which prevents an overt immune response. As a result, $\mathrm{Pa}$ can disseminate to other areas of the host and cause infection through up-regulation of virulence factors secreted by the other four secretion systems, or as in the case of a chronic infection, can mutate and produce alginate which allows the bacteria to survive within a biofilm (Fig. 6).

Future studies investigating the role of host cell migration properties should focus on: (1) further clarifying how the balance between Rho and Rac1 affects Pa-T3S in other cell lines; (2) confirming the role of phosphorylated ERMs and actin linkage to the membrane in Pa-T3S sensitivity through cell line knockout studies of ezrin, the main ERM protein found in epithelial cells; and (3) using an animal model to determine how ExoS GAP and ADPRT activities contribute to the establishment of infection and dissemination of $\mathrm{Pa}$ through the host. 


\section{REFERENCES}


1. Abd, H., B. Wretlind, A. Saeed, E. Idsund, K. Hultenby, and G. Sandstrom. 2008. Pseudomonas aeruginosa Utilises Its Type III Secretion System to Kill the Free-Living Amoeba Acanthamoeba castellanii. J Eukaryot Microbiol 55:235-243.

2. Ader, F., R. Le Berre, K. Faure, P. Gosset, O. Epaulard, B. Toussaint, B. Polack, E. Nowak, N. B. Viget, E. Kipnis, and B. P. Guery. 2005. Alveolar response to Pseudomonas aeruginosa: role of the type III secretion system. Infect Immun 73:42634271.

3. Agrain, C., I. Callebaut, L. Journet, I. Sorg, C. Paroz, L. J. Mota, and G. R. Cornelis. 2005. Characterization of a Type III secretion substrate specificity switch (T3S4) domain in YscP from Yersinia enterocolitica. Mol Microbiol 56:54-67.

4. Aili, M., E. L. Isaksson, S. E. Carlsson, H. Wolf-Watz, R. Rosqvist, and M. S. Francis. 2008. Regulation of Yersinia Yop-effector delivery by translocated YopE. Int J Med Microbiol 298:183-192.

5. Aili, M., E. L. Isaksson, B. Hallberg, H. Wolf-Watz, and R. Rosqvist. 2006. Functional analysis of the YopE GTPase-activating protein (GAP) activity of Yersinia pseudotuberculosis. Cell Microbiol 8:1020-1033.

6. Akeda, Y., and J. E. Galan. 2004. Genetic analysis of the Salmonella enterica type III secretion-associated ATPase InvC defines discrete functional domains. J Bacteriol 186:2402-2412.

7. Alexander, S., G. E. Koehl, M. Hirschberg, E. K. Geissler, and P. Friedl. 2008. Dynamic imaging of cancer growth and invasion: a modified skin-fold chamber model. Histochem Cell Biol 130:1147-1154.

8. Alfonso, E., S. Mandelbaum, M. J. Fox, and R. K. Forster. 1986. Ulcerative keratitis associated with contact lens wear. Am J Ophthalmol 101:429-433. 
9. Allmond, L. R., T. J. Karaca, V. N. Nguyen, T. Nguyen, J. P. Wiener-Kronish, and T. Sawa. 2003. Protein binding between PcrG-PcrV and PcrH-PopB/PopD encoded by the pcrGVH-popBD operon of the Pseudomonas aeruginosa type III secretion system. Infect Immun 71:2230-2233.

10. Aloush, V., S. Navon-Venezia, Y. Seigman-Igra, S. Cabili, and Y. Carmeli. 2006. Multidrug-resistant Pseudomonas aeruginosa: risk factors and clinical impact. Antimicrob Agents Chemother 50:43-48.

11. Altan, Z. M., and G. Fenteany. 2004. c-Jun N-terminal kinase regulates lamellipodial protrusion and cell sheet migration during epithelial wound closure by a gene expression-independent mechanism. Biochem Biophys Res Commun 322:56-67.

12. Amano, M., K. Chihara, K. Kimura, Y. Fukata, N. Nakamura, Y. Matsuura, and K. Kaibuchi. 1997. Formation of actin stress fibers and focal adhesions enhanced by Rhokinase. Science 275:1308-1311.

13. Anderson, G. G., S. Moreau-Marquis, B. A. Stanton, and G. A. O'Toole. 2008. In vitro analysis of tobramycin-treated Pseudomonas aeruginosa biofilms on cystic fibrosisderived airway epithelial cells. Infect Immun 76:1423-1433.

14. Anderson, G. G., T. L. Yahr, R. R. Lovewell, and G. A. O'Toole. 2010. The Pseudomonas aeruginosa magnesium transporter MgtE inhibits transcription of the type III secretion system. Infect Immun 78:1239-1249.

15. Angus, A. A., D. J. Evans, J. T. Barbieri, and S. M. Fleiszig. 2010. The ADPribosylation domain of Pseudomonas aeruginosa ExoS is required for membrane bleb niche formation and bacterial survival within epithelial cells. Infect Immun 78:4500-4510.

16. Apodaca, G., M. Bomsel, R. Lindstedt, J. Engel, D. Frank, K. E. Mostov, and J. Wiener-Kronish. 1995. Characterization of Pseudomonas aeruginosa-induced MDCK cell injury: glycosylation-defective host cells are resistant to bacterial killing. Infect Immun 63:1541-1551. 
17. Bacallao, R., C. Antony, C. Dotti, E. Karsenti, E. H. Stelzer, and K. Simons. 1989. The subcellular organization of Madin-Darby canine kidney cells during the formation of a polarized epithelium. J Cell Biol 109:2817-2832.

18. Banwart, B., M. L. Splaingard, P. M. Farrell, M. J. Rock, P. L. Havens, J. Moss, M. E. Ehrmantraut, D. W. Frank, and J. T. Barbieri. 2002. Children with cystic fibrosis produce an immune response against exoenzyme S, a type III cytotoxin of Pseudomonas aeruginosa. J Infect Dis 185:269-270.

19. Barber, D. F., A. Bartolomé, C. Hernandez, J. M. Flores, C. Redondo, C. FernandezArias, M. Camps, T. Rückle, M. K. Schwarz, S. Rodríguez, C. Martinez-A, D. Balomenos, C. Rommel, and A. C. Carrera. 2005. PI3Ky inhibition blocks glomerulonephritis and extends lifespan in a mouse model of systemic lupus. Nat. Med. 11:933-935.

20. Barbieri, J. T., and J. Sun. 2004. Pseudomonas aeruginosa ExoS and ExoT. Rev Physiol Biochem Pharmacol 152:79-92.

21. Bashour, A. M., A. T. Fullerton, M. J. Hart, and G. S. Bloom. 1997. IQGAP1, a Racand Cdc42-binding protein, directly binds and cross-links microfilaments. J Cell Biol. 137:1555-1566.

22. Bergman, T., S. Hakansson, A. Forsberg, L. Norlander, A. Macellaro, A. Backman, I. Bolin, and H. Wolf-Watz. 1991. Analysis of the V antigen IcrGVH-yopBD operon of Yersinia pseudotuberculosis: evidence for a regulatory role of LcrH and LcrV. J Bacteriol 173:1607-1616.

23. Bert, F., E. Maubec, B. Bruneau, P. Berry, and N. Lambert-Zechovsky. 1998. Multiresistant Pseudomonas aeruginosa outbreak associated with contaminated tap water in a neurosurgery intensive care unit. J Hosp Infect 39:53-62.

24. Biering-Sorensen, F. 2002. Urinary tract infection in individuals with spinal cord lesion. Curr Opin Urol 12:45-49. 
25. Bjorn, M. J., O. R. Pavlovskis, M. R. Thompson, and B. H. Iglewski. 1979. Production of exoenzyme $\mathbf{S}$ during Pseudomonas aeruginosa infections of burned mice. Infect Immun 24:837-842.

26. Black, D. S., and J. B. Bliska. 2000. The RhoGAP activity of the Yersinia pseudotuberculosis cytotoxin YopE is required for antiphagocytic function and virulence. Mol Microbiol 37:515-527.

27. Bleves, S., V. Viarre, R. Salacha, G. P. Michel, A. Filloux, and R. Voulhoux. 2010. Protein secretion systems in Pseudomonas aeruginosa: A wealth of pathogenic weapons. Int J Med Microbiol 300:534-543.

28. Blocker, A., P. Gounon, E. Larquet, K. Niebuhr, V. Cabiaux, C. Parsot, and P. Sansonetti. 1999. The tripartite type III secreton of Shigella flexneri inserts IpaB and IpaC into host membranes. J Cell Biol 147:683-693.

29. Blocker, A., N. Jouihri, E. Larquet, P. Gounon, F. Ebel, C. Parsot, P. Sansonetti, and A. Allaoui. 2001. Structure and composition of the Shigella flexneri "needle complex", a part of its type III secreton. Mol Microbiol 39:652-663.

30. Blocker, A., K. Komoriya, and S. Aizawa. 2003. Type III secretion systems and bacterial flagella: insights into their function from structural similarities. Proc Natl Acad Sci U S A 100:3027-3030.

31. Blocker, A. J., J. E. Deane, A. K. Veenendaal, P. Roversi, J. L. Hodgkinson, S. Johnson, and S. M. Lea. 2008. What's the point of the type III secretion system needle? Proc Natl Acad Sci U S A 105:6507-6513.

32. Borm, B., R. P. Requardt, V. Herzog, and G. Kirfel. 2005. Membrane ruffles in cell migration: indicators of inefficient lamellipodia adhesion and compartments of actin filament reorganization. Exp Cell Res 302:83-95. 
33. Bridge, D. R., M. J. Novotny, E. R. Moore, and J. C. Olson. 2010. Role of host cell polarity and leading edge properties in Pseudomonas type III secretion. Microbiology 156:356-373.

34. Brodsky, I. E., N. W. Palm, S. Sadanand, M. B. Ryndak, F. S. Sutterwala, R. A. Flavell, J. B. Bliska, and R. Medzhitov. 2010. A Yersinia effector protein promotes virulence by preventing inflammasome recognition of the type III secretion system. Cell Host Microbe 7:376-387.

35. Broms, J. E., A. L. Forslund, A. Forsberg, and M. S. Francis. 2003. PcrH of Pseudomonas aeruginosa is essential for secretion and assembly of the type III translocon. J Infect Dis 188:1909-1921.

36. Broz, P., C. A. Mueller, S. A. Muller, A. Philippsen, I. Sorg, A. Engel, and G. R. Cornelis. 2007. Function and molecular architecture of the Yersinia injectisome tip complex. Mol Microbiol 65:1311-1320.

37. Brutinel, E. D., C. A. Vakulskas, K. M. Brady, and T. L. Yahr. 2008. Characterization of ExsA and of ExsA-dependent promoters required for expression of the Pseudomonas aeruginosa type III secretion system. Mol Microbiol 68:657-671.

38. Bucior, I., K. Mostov, and J. N. Engel. 2010. Pseudomonas aeruginosa-mediated damage requires distinct receptors at the apical and basolateral surfaces of the polarized epithelium. Infect Immun 78:939-953.

39. Burrows, T. W. 1956. An antigen determining virulence in Pasteurella pestis. Nature $177: 426-427$.

40. Caiazza, N. C., J. H. Merritt, K. M. Brothers, and G. A. O'Toole. 2007. Inverse regulation of biofilm formation and swarming motility by Pseudomonas aeruginosa PA14. J Bacteriol 189:3603-3612. 
41. Camps, M., T. Rückle, H. Ji, V. Ardissone, F. Rintelen, J. Shaw, C. Ferrandi, C. Chabert, C. Gillieron, B. Françon, T. Martin, D. Gretener, D. Perrin, D. Leroy, P.-A. Vitte, E. Hirsch, M. P. Wymann, R. Cirillo, M. K. Schwarz, and C. Rommel. 2005. Blockade of PI3K suppresses joint inflammation and damage in mouse models of rheumatoid arthritis. Nat Med. 11:936-943.

42. Carmeli, Y., N. Troillet, G. M. Eliopoulos, and M. H. Samore. 1999. Emergence of antibiotic-resistant Pseudomonas aeruginosa: comparison of risks associated with different antipseudomonal agents. Antimicrob Agents Chemother 43:1379-1382.

43. Cheng, K. H., S. L. Leung, H. W. Hoekman, W. H. Beekhuis, P. G. Mulder, A. J. Geerards, and A. Kijlstra. 1999. Incidence of contact-lens-associated microbial keratitis and its related morbidity. Lancet 354:181-185.

44. Chitkara, Y. K., and T. C. Feierabend. 1981. Endogenous and exogenous infection with Pseudomonas aeruginosa in a burns unit. Int Surg 66:237-240.

45. Cisz, M., P. C. Lee, and A. Rietsch. 2008. ExoS controls the cell contact-mediated switch to effector secretion in Pseudomonas aeruginosa. J Bacteriol 190:2726-2738.

46. Coburn, J., A. V. Kane, L. Feig, and D. M. Gill. 1991. Pseudomonas aeruginosa exoenzyme $S$ requires a eukaryotic protein for ADP-ribosyltransferase activity. J Biol Chem 266:6438-6446.

47. Collins, S. J., F. W. Ruscetti, R. E. Gallagher, and R. C. Gallo. 1978. Terminal differentiation of human promyelocyitc leukemia cells induced by dimethyl sulfoxide and other polar compounds. Proc. Natl. Acad. Sci. USA 75:2458-2462.

48. Corech, R., A. Rao, A. Laxova, J. Moss, M. J. Rock, Z. Li, M. R. Kosorok, M. L. Splaingard, P. M. Farrell, and J. T. Barbieri. 2005. Early immune response to the components of the type III system of Pseudomonas aeruginosa in children with cystic fibrosis. J Clin Microbiol 43:3956-3962. 
49. Cornelis, G. R. 2010. The type III secretion injectisome, a complex nanomachine for intracellular 'toxin' delivery. Biol Chem 391:745-751.

50. Cornelis, G. R., T. Biot, C. Lambert de Rouvroit, T. Michiels, B. Mulder, C. Sluiters, M.-P. Sory, M. Van Bouchaute, and J.-C. Vanooteghem. 1989. The Yersinia Yop regulon. Mol. Microbiol. 3:1455-1459.

51. Cornelis, G. R., and H. Wolf-Watz. 1997. The Yersinia Yop virulon: a bacterial system for subverting eukaryotic cells. Mol Microbiol 23:861-867.

52. Cowell, B. A., D. Y. Chen, D. W. Frank, A. J. Vallis, and S. M. Fleiszig. 2000. ExoT of cytotoxic Pseudomonas aeruginosa prevents uptake by corneal epithelial cells. Infect Immun 68:403-406.

53. Cowell, B. A., D. J. Evans, and S. M. Fleiszig. 2005. Actin cytoskeleton disruption by ExoY and its effects on Pseudomonas aeruginosa invasion. FEMS Microbiol Lett 250:71-76.

54. Dabbous, M., M. M. Jefferson, L. Haney, and E. L. Thomas. 2011. Biomarkers of metastatic potential in cultured adenocarcinoma clones. Clin Exp Metastasis 28:101111.

55. Dacheux, D., I. Attree, and B. Toussaint. 2001. Expression of ExsA in trans confers type III secretion system-dependent cytotoxicity on noncytotoxic Pseudomonas aeruginosa cystic fibrosis isolates. Infect Immun 69:538-542.

56. Dacheux, D., J. Goure, J. Chabert, Y. Usson, and I. Attree. 2001. Pore-forming activity of type III system-secreted proteins leads to oncosis of Pseudomonas aeruginosa-infected macrophages. Mol Microbiol 40:76-85.

57. Dacheux, D., B. Toussaint, M. Richard, G. Brochier, J. Croize, and I. Attree. 2000. Pseudomonas aeruginosa cystic fibrosis isolates induce rapid, type III secretiondependent, but ExoU-independent, oncosis of macrophages and polymorphonuclear neutrophils. Infect Immun 68:2916-2924. 
58. Daniell, S. J., N. Takahashi, R. Wilson, D. Friedberg, I. Rosenshine, F. P. Booy, R. K. Shaw, S. Knutton, G. Frankel, and S. Aizawa. 2001. The filamentous type III secretion translocon of enteropathogenic Escherichia coli. Cell Microbiol 3:865-871.

59. Darwin, K. H., and V. L. Miller. 2001. Type III secretion chaperone-dependent regulation: activation of virulence genes by SicA and InvF in Salmonella typhimurium. EMBO J 20:1850-1862.

60. Dasgupta, N., G. L. Lykken, M. C. Wolfgang, and T. L. Yahr. 2004. A novel anti-antiactivator mechanism regulates expression of the Pseudomonas aeruginosa type III secretion system. Mol Microbiol 53:297-308.

61. Davies, S. P., H. Reddy, M. Caivano, and P. Cohen. 2000. Specificity and mechanism of action of some commonly used protein kinase inhibitors. Biochem J. 351:95-105.

62. de Bentzmann, S., P. Roger, F. Dupuit, O. Bajolet-Laudinat, C. Fuchey, M. C. Plotkowski, and E. Puchelle. 1996. Asialo GM1 is a receptor for Pseudomonas aeruginosa adherence to regenerating respiratory epithelial cells. Infect Immun 64:15821588.

63. Deane, J. E., P. Roversi, F. S. Cordes, S. Johnson, R. Kenjale, S. Daniell, F. Booy, W. D. Picking, W. L. Picking, A. J. Blocker, and S. M. Lea. 2006. Molecular model of a type III secretion system needle: Implications for host-cell sensing. Proc Natl Acad Sci U S A 103:12529-12533.

64. Deng, Q., and J. T. Barbieri. 2008. Modulation of host cell endocytosis by the type III cytotoxin, Pseudomonas ExoS. Traffic 9:1948-1957.

65. Deng, Q., and J. T. Barbieri. 2008. Molecular mechanisms of the cytotoxicity of ADPribosylating toxins. Annu Rev Microbiol 62:271-288.

66. Deng, Q., J. Sun, and J. T. Barbieri. 2005. Uncoupling Crk signal transduction by Pseudomonas exoenzyme T. J Biol Chem 280:35953-35960. 
67. Derewenda, U., A. Mateja, Y. Devedjiev, K. M. Routzahn, A. G. Evdokimov, Z. S. Derewenda, and D. S. Waugh. 2004. The structure of Yersinia pestis V-antigen, an essential virulence factor and mediator of immunity against plague. Structure 12:301306.

68. Dewoody, R., P. M. Merritt, A. S. Houppert, and M. M. Marketon. 2011. YopK regulates the Yersinia pestis type III secretion system from within host cells. Mol Microbiol 79:1445-1461.

69. DiNovo, A. A., K. L. Schey, W. S. Vachon, E. M. McGuffie, J. C. Olson, and T. S. Vincent. 2006. ADP-ribosylation of cyclophilin A by Pseudomonas aeruginosa exoenzyme S. Biochemistry 45:4664-4673.

70. Edmonds, B. T., J. Wyckoff, Y. G. Yeung, Y. Wang, E. R. Stanley, J. Jones, J. Segall, and J. Condeelis. 1996. Elongation factor-1 alpha is an overexpressed actin binding protein in metastatic rat mammary adenocarcinoma. J Cell Sci 109 ( Pt 11):2705-2714.

71. El-Sibai, M., P. Nalbant, H. Pang, R. J. Flinn, C. Sarmiento, F. Macaluso, M. Cammer, J. S. Condeelis, K. M. Hahn, and J. M. Backer. 2007. Cdc42 is required for EGF-stimulated protrusion and motility in MTLn3 carcinoma cells. J Cell Sci 120:34653474.

72. El-Sibai, M., O. Pertz, H. Pang, S. C. Yip, M. Lorenz, M. Symons, J. S. Condeelis, K. M. Hahn, and J. M. Backer. 2008. RhoA/ROCK-mediated switching between Cdc42and Rac1-dependent protrusion in MTLn3 carcinoma cells. Exp Cell Res 314:15401552.

73. El-Solh, A. A., A. Hattemer, A. R. Hauser, A. Alhajhusain, and H. Vora. 2011. Clinical outcomes of type III Pseudomonas aeruginosa bacteremia. Crit Care Med. 
74. El Amari, E. B., E. Chamot, R. Auckenthaler, J. C. Pechere, and C. Van Delden. 2001. Influence of previous exposure to antibiotic therapy on the susceptibility pattern of Pseudomonas aeruginosa bacteremic isolates. Clin Infect Dis 33:1859-1864.

75. El Solh, A. A., M. E. Akinnusi, J. P. Wiener-Kronish, S. V. Lynch, L. A. Pineda, and K. Szarpa. 2008. Persistent infection with Pseudomonas aeruginosa in ventilatorassociated pneumonia. Am J Respir Crit Care Med 178:513-519.

76. Emam, A., W. G. Carter, and C. Lingwood. 2010. Glycolipid-Dependent, Protease Sensitive Internalization of Pseudomonas aeruginosa Into Cultured Human Respiratory Epithelial Cells. Open Microbiol J 4:106-115.

77. Evans, D. J., D. W. Frank, V. Finck-Barbancon, C. Wu, and S. M. Fleiszig. 1998. Pseudomonas aeruginosa invasion and cytotoxicity are independent events, both of which involve protein tyrosine kinase activity. Infect Immun 66:1453-1459.

78. Fackler, O. T., and R. Grosse. 2008. Cell motility through plasma membrane blebbing. J Cell Biol 181:879-884.

79. Farooqui, R., and G. Fenteany. 2005. Multiple rows of cells behind an epithelial wound edge extend cryptic lamellipodia to collectively drive cell-sheet movement. J Cell Sci 118:51-63.

80. Faudry, E., G. Vernier, E. Neumann, V. Forge, and I. Attree. 2006. Synergistic pore formation by type III toxin translocators of Pseudomonas aeruginosa. Biochemistry 45:8117-8123.

81. Feldman, M., R. Bryan, S. Rajan, L. Scheffler, S. Brunnert, H. Tang, and A. Prince. 1998. Role of flagella in pathogenesis of Pseudomonas aeruginosa pulmonary infection. Infect Immun 66:43-51.

82. Feltman, H., G. Schulert, S. Khan, M. Jain, L. Peterson, and A. R. Hauser. 2001. Prevalence of type III secretion genes in clinical and environmental isolates of Pseudomonas aeruginosa. Microbiology 147:2659-2669. 
83. Fenteany, G., P. A. Janmey, and T. P. Stossel. 2000. Signaling pathways and cell mechanics involved in wound closure by epithelial cell sheets. Curr Biol 10:831-838.

84. Fields, K. A., G. V. Plano, and S. C. Straley. 1994. A low-Ca2+ response (LCR) secretion (ysc) locus lies within the IcrB region of the LCR plasmid in Yersinia pestis. $J$ Bacteriol 176:569-579.

85. Finck-Barbancon, V., J. Goranson, L. Zhu, T. Sawa, J. P. Wiener-Kronish, S. M. Fleiszig, C. Wu, L. Mende-Mueller, and D. W. Frank. 1997. ExoU expression by Pseudomonas aeruginosa correlates with acute cytotoxicity and epithelial injury. Mol Microbiol 25:547-557.

86. Fleiszig, S. M., N. Efron, and G. B. Pier. 1992. Extended contact lens wear enhances Pseudomonas aeruginosa adherence to human corneal epithelium. Invest Ophthalmol Vis Sci 33:2908-2916.

87. Fleiszig, S. M., D. J. Evans, N. Do, V. Vallas, S. Shin, and K. E. Mostov. 1997. Epithelial cell polarity affects susceptibility to Pseudomonas aeruginosa invasion and cytotoxicity. Infect Immun 65:2861-2867.

88. Fleiszig, S. M., J. P. Wiener-Kronish, H. Miyazaki, V. Vallas, K. E. Mostov, D. Kanada, T. Sawa, T. S. Yen, and D. W. Frank. 1997. Pseudomonas aeruginosamediated cytotoxicity and invasion correlate with distinct genotypes at the loci encoding exoenzyme S. Infect Immun 65:579-586.

89. Fleiszig, S. M., T. S. Zaidi, E. L. Fletcher, M. J. Preston, and G. B. Pier. 1994. Pseudomonas aeruginosa invades corneal epithelial cells during experimental infection. Infect Immun 62:3485-3493.

90. Fleiszig, S. M., T. S. Zaidi, M. J. Preston, M. Grout, D. J. Evans, and G. B. Pier. 1996. Relationship between cytotoxicity and corneal epithelial cell invasion by clinical isolates of Pseudomonas aeruginosa. Infect Immun 64:2288-2294. 
91. Fleiszig, S. M. J., D. J. Evans, N. Do, S. Shin, and K. E. Mostov. 1997. Epithelial cell polarity affects susceptibility to Pseudomonas aeruginosa invasion and cytotoxicity. Infect. Immun. 65:2861-2867.

92. Frank, D. W. 1997. The exoenzyme S regulon of Pseudomonas aeruginosa. Mol Microbiol 26:621-629.

93. Frank, D. W., and B. H. Iglewski. 1991. Cloning and sequence analysis of a transregulatory locus required for exoenzyme $\mathrm{S}$ synthesis in Pseudomonas aeruginosa. J Bacteriol 173:6460-6468.

94. Franklin, A. L., T. Todd, G. Gurman, D. Black, P. M. Mankinen-Irvin, and R. T. Irvin. 1987. Adherence of Pseudomonas aeruginosa to cilia of human tracheal epithelial cells. Infect Immun 55:1523-1525.

95. Fraylick, J. E., J. R. La Rocque, T. S. Vincent, and J. C. Olson. 2001. Independent and coordinate effects of ADP-ribosyltransferase and GTPase-activating activities of exoenzyme S on HT-29 epithelial cell function. Infect Immun 69:5318-5328.

96. Fraylick, J. E., M. J. Riese, T. S. Vincent, J. T. Barbieri, and J. C. Olson. 2002. ADPribosylation and functional effects of Pseudomonas exoenzyme $S$ on cellular RalA. Biochemistry 41:9680-9687.

97. Fraylick, J. E., E. A. Rucks, D. M. Greene, T. S. Vincent, and J. C. Olson. 2002. Eukaryotic cell determination of ExoS ADP-ribosyltransferase substrate specificity. Biochem Biophys Res Commun 291:91-100.

98. Friedl, P., and D. Gilmour. 2009. Collective cell migration in morphogenesis, regeneration and cancer. Nat Rev Mol Cell Biol 10:445-457.

99. FriedI, P., and K. Wolf. 2010. Plasticity of cell migration: a multiscale tuning model. J Cell Biol 188:11-19. 
100. Frithz-Lindsten, E., Y. Du, R. Rosqvist, and A. Forsberg. 1997. Intracellular targeting of exoenzyme $S$ of Pseudomonas aeruginosa via type III-dependent translocation induces phagocytosis resistance, cytotoxicity and disruption of actin microfilaments. Mol Microbiol 25:1125-1139.

101. Frithz-Lindsten, E., A. Holmstrom, L. Jacobsson, M. Soltani, J. Olsson, R. Rosqvist, and A. Forsberg. 1998. Functional conservation of the effector protein translocators $\mathrm{PopB} / \mathrm{YopB}$ and PopD/YopD of Pseudomonas aeruginosa and Yersinia pseudotuberculosis. Mol Microbiol 29:1155-1165.

102. Fu, H., J. Coburn, and R. J. Collier. 1993. The eukaryotic host factor that activates exoenzyme $S$ of Pseudomonas aeruginosa is a member of the 14-3-3 protein family. Proc Natl Acad Sci U S A 90:2320-2324.

103. Fu, Y., and J. E. Galan. 1998. Identification of a specific chaperone for SptP, a substrate of the centisome 63 type III secretion system of Salmonella typhimurium. J Bacteriol 180:3393-3399.

104. Fukata, M., T. Watanabe, J. Noritake, M. Nakagawa, M. Yamaga, S. Kuroda, Y. Matsuura, A. Iwamatsu, F. Perez, and K. Kaibuchi. 2002. Rac1 and Cdc42 capture microtubules through IQGAP1 and CLIP-170. Cell 109:873-885.

105. Galentine, P. G., E. J. Cohen, P. R. Laibson, C. P. Adams, R. Michaud, and J. J. Arentsen. 1984. Corneal ulcers associated with contact lens wear. Arch Ophthalmol 102:891-894.

106. Ganesan, A. K., D. W. Frank, R. P. Misra, G. Schmidt, and J. T. Barbieri. 1998. Pseudomonas aeruginosa exoenzyme S ADP-ribosylates Ras at multiple sites. J Biol Chem 273:7332-7337.

107. Gao, Y., J. B. Dickerson, F. Guo, J. Zheng, and Y. Zheng. 2004. Rational design and characterization of a Rac GTPase-specific small molecule inhibitor Proc Natl Acad Sci U S A. 101:7618-7623 
108. Garrity-Ryan, L., B. Kazmierczak, R. Kowal, J. Comolli, A. Hauser, and J. N. Engel. 2000. The arginine finger domain of ExoT contributes to actin cytoskeleton disruption and inhibition of internalization of Pseudomonas aeruginosa by epithelial cells and macrophages. Infect Immun 68:7100-7113.

109. Garrity-Ryan, L., S. Shafikhani, P. Balachandran, L. Nguyen, J. Oza, T. Jakobsen, J. Sargent, X. Fang, S. Cordwell, M. A. Matthay, and J. N. Engel. 2004. The ADP ribosyltransferase domain of Pseudomonas aeruginosa ExoT contributes to its biological activities. Infect Immun 72:546-558.

110. Gauthier, A., and B. B. Finlay. 2003. Translocated intimin receptor and its chaperone interact with ATPase of the type III secretion apparatus of enteropathogenic Escherichia coli. J Bacteriol 185:6747-6755.

111. Gebus, C., E. Faudry, Y. S. Bohn, E. Sylvie, and I. Attree. 2008. Oligomerization of PcrV and LcrV, protective antigens of Pseudomonas aeruginosa and Yersinia pestis. J Biol Chem 283:23940-23949.

112. Gekara, N. O., T. Jacobs, T. Chakraborty, and S. Weiss. 2005. The cholesteroldependent cytolysin listeriolysin $\mathrm{O}$ aggregates rafts via oligomerization. Cell Microbiol 7:1345-1356.

113. Gerceker, A. A., T. Zaidi, P. Marks, D. E. Golan, and G. B. Pier. 2000. Impact of heterogeneity within cultured cells on bacterial invasion: analysis of Pseudomonas aeruginosa and Salmonella enterica serovar typhi entry into MDCK cells by using a green fluorescent protein-labelled cystic fibrosis transmembrane conductance regulator receptor. Infect Immun 68:861-870.

114. Ghosh, P. 2004. Process of protein transport by the type III secretion system. Microbiol Mol Biol Rev 68:771-795. 
115. Goeckeler, Z. M., and R. B. Wysolmerski. 1995. Myosin light chain kinase-regulated endothelial cell contraction: the relationship between isometric tension, actin polymerization, and myosin phosphorylation. J Cell Biol 130:613-627.

116. Goehring, U. M., G. Schmidt, K. J. Pederson, K. Aktories, and J. T. Barbieri. 1999. The N-terminal domain of Pseudomonas aeruginosa exoenzyme $S$ is a GTPaseactivating protein for Rho GTPases. J Biol Chem 274:36369-36372.

117. Goodman, A. L., B. Kulasekara, A. Rietsch, D. Boyd, R. S. Smith, and S. Lory. 2004. A signaling network reciprocally regulates genes associated with acute infection and chronic persistence in Pseudomonas aeruginosa. Dev Cell 7:745-754.

118. Goure, J., P. Broz, O. Attree, G. R. Cornelis, and I. Attree. 2005. Protective anti-V antibodies inhibit Pseudomonas and Yersinia translocon assembly within host membranes. J Infect Dis 192:218-225.

119. Goure, J., A. Pastor, E. Faudry, J. Chabert, A. Dessen, and I. Attree. 2004. The V antigen of Pseudomonas aeruginosa is required for assembly of the functional PopB/PopD translocation pore in host cell membranes. Infect Immun 72:4741-4750.

120. Govan, J. R., and V. Deretic. 1996. Microbial pathogenesis in cystic fibrosis: mucoid Pseudomonas aeruginosa and Burkholderia cepacia. Microbiol Rev 60:539-574.

121. Grinnell, F. 2008. Fibroblast mechanics in three-dimensional collagen matrices. J Bodyw Mov Ther 12:191-193.

122. Gupta, S. K., R. S. Berk, S. Masinick, and L. D. Hazlett. 1994. Pili and lipopolysaccharide of Pseudomonas aeruginosa bind to the glycolipid asialo GM1. Infect Immun 62:4572-4579.

123. Ha, U. H., J. Kim, H. Badrane, J. Jia, H. V. Baker, D. Wu, and S. Jin. 2004. An in vivo inducible gene of Pseudomonas aeruginosa encodes an anti-ExsA to suppress the type III secretion system. Mol Microbiol 54:307-320. 
124. Hamood, A. N., J. A. Griswold, and C. M. Duhan. 1996. Production of extracellular virulence factors by Pseudomonas aeruginosa isolates obtained from tracheal, urinary tract, and wound infections. J Surg Res 61:425-432.

125. Hancock, R. E., L. M. Mutharia, L. Chan, R. P. Darveau, D. P. Speert, and G. B. Pier. 1983. Pseudomonas aeruginosa isolates from patients with cystic fibrosis: a class of serum-sensitive, nontypable strains deficient in lipopolysaccharide $\mathrm{O}$ side chains. Infect Immun 42:170-177.

126. Harris, A., C. Torres-Viera, L. Venkataraman, P. DeGirolami, M. Samore, and Y. Carmeli. 1999. Epidemiology and clinical outcomes of patients with multiresistant Pseudomonas aeruginosa. Clin Infect Dis 28:1128-1133.

127. Hart, M. J., M. G. Callow, B. Souza, and P. Polakis. 1996. IQGAP1, a calmodulinbinding protein with a rasGAP-related domain, is a potential effector for cdc42Hs. EMBO J. 15:2997-3005.

128. Hauert, A. B., S. Martinelli, C. Marone, and V. Niggli. 2002. Differentiated HL-60 cells are a valid model system for the analysis of human neutrophil migration and chemotaxis. Int J Biochem Cell Biol. 34:838-854.

129. Hauser, A. R. 2009. The type III secretion system of Pseudomonas aeruginosa: infection by injection. Nat Rev Microbiol 7:654-665.

130. Hauser, A. R., E. Cobb, M. Bodi, D. Mariscal, J. Valles, J. N. Engel, and J. Rello. 2002. Type III protein secretion is associated with poor clinical outcomes in patients with ventilator-associated pneumonia caused by Pseudomonas aeruginosa. Crit Care Med 30:521-528.

131. Hauser, A. R., S. Fleiszig, P. J. Kang, K. Mostov, and J. N. Engel. 1998. Defects in type III secretion correlate with internalization of Pseudomonas aeruginosa by epithelial cells. Infect Immun 66:1413-1420. 
132. Hayward, R. D., R. J. Cain, E. J. McGhie, N. Phillips, M. J. Garner, and V. Koronakis. 2005. Cholesterol binding by the bacterial type III translocon is essential for virulence effector delivery into mammalian cells. Mol Microbiol 56:590-603.

133. Hegerfeldt, Y., M. Tusch, E. B. Brocker, and P. Friedl. 2002. Collective cell movement in primary melanoma explants: plasticity of cell-cell interaction, beta1-integrin function, and migration strategies. Cancer Res 62:2125-2130.

134. Henriksson, M. L., M. S. Francis, A. Peden, M. Aili, K. Stefansson, R. Palmer, A. Aitken, and B. Hallberg. 2002. A nonphosphorylated 14-3-3 binding motif on exoenzyme $S$ that is functional in vivo. Eur J Biochem 269:4921-4929.

135. Henriksson, M. L., R. Rosqvist, M. Telepnev, H. Wolf-Watz, and B. Hallberg. 2000. Ras effector pathway activation by epidermal growth factor is inhibited in vivo by exoenzyme S ADP-ribosylation of Ras. Biochem J 347 Pt 1:217-222.

136. Henriksson, M. L., C. Sundin, A. L. Jansson, A. Forsberg, R. H. Palmer, and B. Hallberg. 2002. Exoenzyme S shows selective ADP-ribosylation and GTPase-activating protein (GAP) activities towards small GTPases in vivo. Biochem J 367:617-628.

137. Henriksson, M. L., U. Troller, and B. Hallberg. 2000. 14-3-3 proteins are required for the inhibition of Ras by exoenzyme S. Biochem J 349 Pt 3:697-701.

138. Herard, A. L., J. M. Zahm, D. Pierrot, J. Hinnrasky, C. Fuchey, and E. Puchelle. 1996. Epithelial barrier integrity during in vitro wound repair of the airway epithelium. Am J Respir Cell Mol Biol 15:624-632.

139. Hingley, S. T., A. T. Hastie, F. Kueppers, and M. L. Higgins. 1986. Disruption of respiratory cilia by proteases including those of Pseudomonas aeruginosa. Infect Immun 54:379-385. 
140. Hodgkinson, J. L., A. Horsley, D. Stabat, M. Simon, S. Johnson, P. C. da Fonseca, E. P. Morris, J. S. Wall, S. M. Lea, and A. J. Blocker. 2009. Three-dimensional reconstruction of the Shigella T3SS transmembrane regions reveals 12-fold symmetry and novel features throughout. Nat Struct Mol Biol 16:477-485.

141. Hogardt, M., and J. Heesemann. 2010. Adaptation of Pseudomonas aeruginosa during persistence in the cystic fibrosis lung. Int J Med Microbiol 300:557-562.

142. Holder, I. A., A. N. Neely, and D. W. Frank. 2001. PcrV immunization enhances survival of burned Pseudomonas aeruginosa-infected mice. Infect Immun 69:5908-5910.

143. Hota, S., Z. Hirji, K. Stockton, C. Lemieux, H. Dedier, G. Wolfaardt, and M. A. Gardam. 2009. Outbreak of multidrug-resistant Pseudomonas aeruginosa colonization and infection secondary to imperfect intensive care unit room design. Infect Control Hosp Epidemiol 30:25-33.

144. Hotze, E. M., E. M. Wilson-Kubalek, J. Rossjohn, M. W. Parker, A. E. Johnson, and R. K. Tweten. 2001. Arresting pore formation of a cholesterol-dependent cytolysin by disulfide trapping synchronizes the insertion of the transmembrane-sheet from a prepore intermediate. J. Biol. Chem. 276:8261-8268.

145. Huet, C., C. Sahuquillo-Merino, E. Coudrier, and D. Luvard. 1987. Absorptive and mucus-secreting subclones isolated from a multipotent intestinal cell line (HT-29) provide new models for cell polarity and terminal differentiation. J Cell Biol. 105:345-357.

146. Iglewski, B. H., and D. Kabat. 1975. NAD-dependent inhibition of protein synthesis by Pseudomonas aeruginosa toxin. Proc Natl Acad Sci U S A 72:2284-2288.

147. Iglewski, B. H., J. Sadoff, M. J. Bjorn, and E. S. Maxwell. 1978. Pseudomonas aeruginosa exoenzyme $\mathrm{S}$ : an adenosine diphosphate ribosyltransferase distinct from toxin A. Proc Natl Acad Sci U S A 75:3211-3215. 
148. Inocencio, N. M., J. M. Moehring, and T. J. Moehring. 1994. Furin activates Pseudomonas exotoxin A by specific cleavage in vivo and in vitro. J Biol Chem 269:31831-31835.

149. Isshiki, M., J. Ando, K. Yamamoto, T. Fujita, Y. Ying, and R. G. Anderson. 2002. Sites of $\mathrm{Ca}(2+)$ wave initiation move with caveolae to the trailing edge of migrating cells. J Cell Sci 115:475-484.

150. Itoh, R. E., K. Kurokawa, Y. Ohba, H. Yoshizaki, N. Mochizuki, and M. Matsuda. 2002. Activation of rac and cdc42 video imaged by fluorescent resonance energy transfer-based single-molecule probes in the membrane of living cells. Mol Cell Biol 22:6582-6591.

151. Jain, M., D. Ramirez, R. Seshadri, J. F. Cullina, C. A. Powers, G. S. Schulert, M. Bar-Meir, C. L. Sullivan, S. A. McColley, and A. R. Hauser. 2004. Type III secretion phenotypes of Pseudomonas aeruginosa strains change during infection of individuals with cystic fibrosis. J Clin Microbiol 42:5229-5237.

152. Jarvis, W. R., and W. J. Martone. 1992. Predominant pathogens in hospital infections. J Antimicrob Chemother 29 Suppl A:19-24.

153. Journet, L., C. Agrain, P. Broz, and G. R. Cornelis. 2003. The needle length of

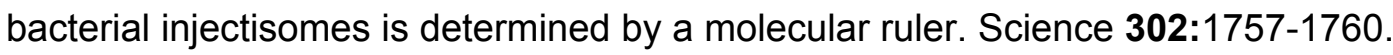

154. Kazmierczak, B. I., and J. N. Engel. 2002. Pseudomonas aeruginosa ExoT acts in vivo as a GTPase-activating protein for RhoA, Rac1, and Cdc42. Infect Immun 70:21982205.

155. Kazmierczak, B. I., T. S. Jou, K. Mostov, and J. N. Engel. 2001. Rho GTPase activity modulates Pseudomonas aeruginosa internalization by epithelial cells. Cell Microbiol 3:85-98.

156. Kazmierczak, B. I., K. Mostov, and J. N. Engel. 2004. Epithelial cell polarity alters Rho-GTPase responses to Pseudomonas aeruginosa. Mol Biol Cell 15:411-419. 
157. Kerr, K. G., and A. M. Snelling. 2009. Pseudomonas aeruginosa: a formidable and ever-present adversary. J Hosp Infect 73:338-344.

158. Kierbel, A., A. Gassama-Diagne, K. Mostov, and J. N. Engel. 2005. The phosphoinositol-3-kinase-protein kinase B/Akt pathway is critical for Pseudomonas aeruginosa strain PAK internalization. Mol Biol Cell 16:2577-2585.

159. Kierbel, A., A. Gassama-Diagne, C. Rocha, L. Radoshevich, J. Olson, K. Mostov, and J. Engel. 2007. Pseudomonas aeruginosa exploits a PIP3-dependent pathway to transform apical into basolateral membrane. J Cell Biol 177:21-27.

160. Kimura, K., M. Ito, M. Amano, K. Chihara, Y. Fukata, M. Nakafuku, B. Yamamori, J. Feng, T. Nakano, K. Okawa, A. Iwamatsu, and K. Kaibuchi. 1996. Regulation of myosin phosphatase by Rho and Rho-associated kinase (Rho-kinase). Science 273:245-248.

161. Kipnis, E., T. Sawa, and J. Wiener-Kronish. 2006. Targeting mechanisms of Pseudomonas aeruginosa pathogenesis. Med Mal Infect 36:78-91.

162. Klinghoffer, R. A., C. Sachsenmaier, J. A. Cooper, and P. Soriano. 1999. Src family kinases are required for integrin but not PDGFR signal transduction. EMBO J 18:24592471.

163. Knight, D. A., V. Finck-Barbancon, S. M. Kulich, and J. T. Barbieri. 1995. Functional domains of Pseudomonas aeruginosa exoenzyme S. Infect Immun 63:3182-3186.

164. Koidou-Tsiligianni, A., E. Alfonso, and R. K. Forster. 1989. Ulcerative keratitis associated with contact lens wear. Am J Ophthalmol 108:64-67.

165. Kounnas, M. Z., R. E. Morris, M. R. Thompson, D. J. FitzGerald, D. K. Strickland, and C. B. Saelinger. 1992. The alpha 2-macroglobulin receptor/low density lipoprotein receptor-related protein binds and internalizes Pseudomonas exotoxin A. J Biol Chem 267:12420-12423. 
166. Krall, R., G. Schmidt, K. Aktories, and J. T. Barbieri. 2000. Pseudomonas aeruginosa ExoT is a Rho GTPase-activating protein. Infect Immun 68:6066-6068.

167. Krall, R., J. Sun, K. J. Pederson, and J. T. Barbieri. 2002. In vivo rho GTPaseactivating protein activity of Pseudomonas aeruginosa cytotoxin ExoS. Infect Immun 70:360-367.

168. Kubori, T., Y. Matsushima, D. Nakamura, J. Uralil, M. Lara-Tejero, A. Sukhan, J. E. Galan, and S. I. Aizawa. 1998. Supramolecular structure of the Salmonella typhimurium type III protein secretion system. Science 280:602-605.

169. Kuchma, S. L., J. P. Connolly, and G. A. O'Toole. 2005. A three-component regulatory system regulates biofilm maturation and type III secretion in Pseudomonas aeruginosa. J Bacteriol 187:1441-1454.

170. Kudoh, I., J. P. Wiener-Kronish, S. Hashimoto, J. F. Pittet, and D. Frank. 1994. Exoproduct secretions of Pseudomonas aeruginosa strains influence severity of alveolar epithelial injury. Am J Physiol 267:L551-556.

171. Kulich, S. M., D. W. Frank, and J. T. Barbieri. 1995. Expression of recombinant exoenzyme S of Pseudomonas aeruginosa. Infect Immun 63:1-8.

172. Kulich, S. M., D. W. Frank, and J. T. Barbieri. 1993. Purification and characterization of exoenzyme S from Pseudomonas aeruginosa 388. Infect Immun 61:307-313.

173. Kurokawa, K., and M. Matsuda. 2005. Localized RhoA activation as a requirement for the induction of membrane ruffling. Mol Biol Cell 16:4294-4303.

174. Laemmli, U. K. 1970. Cleavage of structural proteins during the assembly of the head of bacteriophage T4. Nature 227:680-685.

175. Lafont, F., L. Abrami, and F. G. van der Goot. 2004. Bacterial subversion of lipid rafts. Curr. Opin. Microbiol. 7:4-10. 
176. Lafont, F., and F. G. van der Goot. 2005. Oiling the key hole. Molec Micro. 56:575-577.

177. Lam, J. S., Matewish, J.M., Poon, K.K.H. 2004. Lipopolysaccharides of Pseudomonas, p. 3-53. In J. L. Ramos (ed.), Pseudomonas-Biosynthesis of Macromolecules and Molecular Mechanisms Kluwer Academic/Plenum Publisher, New York.

178. Lammermann, T., B. L. Bader, S. J. Monkley, T. Worbs, R. Wedlich-Soldner, K. Hirsch, M. Keller, R. Forster, D. R. Critchley, R. Fassler, and M. Sixt. 2008. Rapid leukocyte migration by integrin-independent flowing and squeezing. Nature 453:51-55.

179. Lara-Tejero, M., J. Kato, S. Wagner, X. Liu, and J. E. Galan. 2011. A sorting platform determines the order of protein secretion in bacterial type III systems. Science 331:1188-1191.

180. Larbi, A., N. Douziech, A. Khalil, G. Dupuis, S. Gheraïri, K. P. Guérard, and T. Fülöp, Jr. 2004. Effects of methyl-beta-cyclodextrin on T lymphocytes lipid rafts with aging. Exp Gerontol. 39:551-558.

181. Lee, P. C., C. M. Stopford, A. G. Svenson, and A. Rietsch. 2010. Control of effector export by the Pseudomonas aeruginosa type III secretion proteins PcrG and PcrV. Mol Microbiol 75:924-941.

182. Lee, S. H., and J. E. Galan. 2003. InvB is a type III secretion-associated chaperone for the Salmonella enterica effector protein SopE. J Bacteriol 185:7279-7284.

183. Lee, V. T., R. S. Smith, B. Tummler, and S. Lory. 2005. Activities of Pseudomonas aeruginosa effectors secreted by the Type III secretion system in vitro and during infection. Infect Immun 73:1695-1705.

184. Leglise, M. C., G. A. Dent, L. H. Ayscue, and D. W. Ross. 1988. Leukemic cell maturation: phenotypic variability and oncogene expression in HL-60 cells: a review. Blood Cells 13:319-337. 
185. Lepanto, P., D. M. Bryant, J. Rossello, A. Datta, K. E. Mostov, and A. Kierbel. 2011. Pseudomonas aeruginosa interacts with epithelial cells rapidly forming aggregates that are internalized by a Lyn-dependent mechanism. Cell Microbiol 13:1212-1222.

186. Litvak, Y., R. Levin-Klein, M. Avner, and Z. Selinger. 2011. High catalytic efficiency and resistance to denaturing in bacterial Rho GTPase-activating proteins. Biol Chem 392:505-516.

187. Liu, P. V. 1966. The roles of various fractions of Pseudomonas aeruginosa in its pathogenesis. II. Effects of lecithinase and protease. J Infect Dis 116:112-116.

188. Liu, S., S. M. Kulich, and J. T. Barbieri. 1996. Identification of glutamic acid 381 as a candidate active site residue of Pseudomonas aeruginosa exoenzyme S. Biochemistry $35: 2754-2758$.

189. Lodise, T. P., Jr., N. Patel, A. Kwa, J. Graves, J. P. Furuno, E. Graffunder, B. Lomaestro, and J. C. McGregor. 2007. Predictors of 30-day mortality among patients with Pseudomonas aeruginosa bloodstream infections: impact of delayed appropriate antibiotic selection. Antimicrob Agents Chemother 51:3510-3515.

190. Lyczak, J. B., C. L. Cannon, and G. B. Pier. 2000. Establishment of Pseudomonas aeruginosa infection: lessons from a versatile opportunist. Microbes Infect 2:1051-1060.

191. Lyczak, J. B., C. L. Cannon, and G. B. Pier. 2002. Lung infections associated with cystic fibrosis. Clin Microbiol Rev 15:194-222.

192. Mahenthiralingam, E., M. E. Campbell, and D. P. Speert. 1994. Nonmotility and phagocytic resistance of Pseudomonas aeruginosa isolates from chronically colonized patients with cystic fibrosis. Infect Immun 62:596-605.

193. Manes, S., and M.-A. C. 2004. Cholesterol domains regulate the actin cytoskeleton at the leading edge of moving cells. Trends Cell Biol 14:275-278. 
194. Marenne, M. N., L. Journet, L. J. Mota, and G. R. Cornelis. 2003. Genetic analysis of the formation of the Ysc-Yop translocation pore in macrophages by Yersinia enterocolitica: role of LcrV, YscF and YopN. Microb Pathog 35:243-258.

195. Maresso, A. W., M. R. Baldwin, and J. T. Barbieri. 2004. Ezrin/radixin/moesin proteins are high affinity targets for ADP-ribosylation by Pseudomonas aeruginosa ExoS. J Biol Chem 279:38402-38408.

196. Maresso, A. W., Q. Deng, M. S. Pereckas, B. T. Wakim, and J. T. Barbieri. 2007. Pseudomonas aeruginosa ExoS ADP-ribosyltransferase inhibits ERM phosphorylation. Cell Microbiol 9:97-105.

197. Marlovits, T. C., T. Kubori, A. Sukhan, D. R. Thomas, J. E. Galan, and V. M. Unger. 2004. Structural insights into the assembly of the type III secretion needle complex. Science 306:1040-1042.

198. Martinez-Argudo, I., and A. J. Blocker. 2010. The Shigella T3SS needle transmits a signal for MxiC release, which controls secretion of effectors. Mol Microbiol 78:13651378.

199. Mataraza, J. M., M. W. Briggs, Z. Li, A. Entwistle, A. J. Ridley, and D. B. Sacks. 2003. IQGAP1 promotes cell motility and invasion. J Biol Chem. 278:41237-41245.

200. Matsubayashi, Y., M. Ebisuya, S. Honjoh, and E. Nishida. 2004. ERK activation propagates in epithelial cell sheets and regulates their migration during wound healing. Curr Biol 14:731-735.

201. Mattei, P. J., E. Faudry, V. Job, T. Izore, I. Attree, and A. Dessen. 2011. Membrane targeting and pore formation by the type III secretion system translocon. FEBS J 278:414-426.

202. Mavris, M., A. L. Page, R. Tournebize, B. Demers, P. Sansonetti, and C. Parsot. 2002. Regulation of transcription by the activity of the Shigella flexneri type III secretion apparatus. Mol Microbiol 43:1543-1553. 
203. McCaw, M. L., G. L. Lykken, P. K. Singh, and T. L. Yahr. 2002. ExsD is a negative regulator of the Pseudomonas aeruginosa type III secretion regulon. Mol Microbiol 46:1123-1133.

204. McElroy, M. C., J. F. Pittet, S. Hashimoto, L. Allen, J. P. Wiener-Kronish, and L. G. Dobbs. 1995. A type I cell-specific protein is a biochemical marker of epithelial injury in a rat model of pneumonia. Am J Physiol 268:L181-186.

205. McGuffie, E. M., D. W. Frank, T. S. Vincent, and J. C. Olson. 1998. Modification of Ras in eukaryotic cells by Pseudomonas aeruginosa exoenzyme S. Infect Immun 66:2607-2613.

206. McGuffie, E. M., J. E. Fraylick, D. J. Hazen-Martin, T. S. Vincent, and J. C. Olson. 1999. Differential sensitivity of human epithelial cells to Pseudomonas aeruginosa exoenzyme S. Infect Immun 67:3494-3503.

207. Mejia, E., J. B. Bliska, and G. I. Viboud. 2008. Yersinia controls type III effector delivery into host cells by modulating Rho activity. PLoS Pathog 4:e3.

208. Mikkelsen, H., N. J. Bond, M. E. Skindersoe, M. Givskov, K. S. Lilley, and M. Welch. 2009. Biofilms and type III secretion are not mutually exclusive in Pseudomonas aeruginosa. Microbiology 155:687-698.

209. Mittal, R., S. Aggarwal, S. Sharma, S. Chhibber, and K. Harjai. 2009. Urinary tract infections caused by Pseudomonas aeruginosa: a minireview. J Infect Public Health 2:101-111.

210. Moolenaar, R. L., J. M. Crutcher, V. H. San Joaquin, L. V. Sewell, L. C. Hutwagner, L. A. Carson, D. A. Robison, L. M. Smithee, and W. R. Jarvis. 2000. A prolonged outbreak of Pseudomonas aeruginosa in a neonatal intensive care unit: did staff fingernails play a role in disease transmission? Infect Control Hosp Epidemiol 21:80-85.

211. Morihara, K. 1964. Production of Elastase and Proteinase by Pseudomonas Aeruginosa. J Bacteriol 88:745-757. 
212. Moriyama, K., J. P. Wiener-Kronish, and T. Sawa. 2009. Protective effects of affinitypurified antibody and truncated vaccines against Pseudomonas aeruginosa V-antigen in neutropenic mice. Microbiol Immunol 53:587-594.

213. Moss, J., M. E. Ehrmantraut, B. D. Banwart, D. W. Frank, and J. T. Barbieri. 2001. Sera from adult patients with cystic fibrosis contain antibodies to Pseudomonas aeruginosa type III apparatus. Infect Immun 69:1185-1188.

214. Mota, L. J., L. Journet, I. Sorg, C. Agrain, and G. R. Cornelis. 2005. Bacterial injectisomes: needle length does matter. Science 307:1278.

215. Mueller, C. A., P. Broz, and G. R. Cornelis. 2008. The type III secretion system tip complex and translocon. Mol Microbiol 68:1085-1095.

216. Mueller, C. A., P. Broz, S. A. Muller, P. Ringler, F. Erne-Brand, I. Sorg, M. Kuhn, A. Engel, and G. R. Cornelis. 2005. The V-antigen of Yersinia forms a distinct structure at the tip of injectisome needles. Science 310:674-676.

217. Neri, A., D. Welch, T. Kawaguchi, and G. L. Nicolson. 1982. Development and biologic properties of malignant cell sublines and clones of a spontaneously metastasizing rat mammary adenocarcinoma. J Natl Cancer Inst 68:507-517.

218. Nicas, T. I., J. Bradley, J. E. Lochner, and B. H. Iglewski. 1985. The role of exoenzyme S in infections with Pseudomonas aeruginosa. J Infect Dis 152:716-721.

219. Nicas, T. I., and B. H. Iglewski. 1984. Isolation and characterization of transposoninduced mutants of Pseudomonas aeruginosa deficient in production of exoenzyme S. Infect Immun 45:470-474.

220. Nilles, M. L., K. A. Fields, and S. C. Straley. 1998. The V antigen of Yersinia pestis regulates Yop vectorial targeting as well as Yop secretion through effects on YopB and LcrG. J Bacteriol 180:3410-3420.

221. Nobes, C. D., and A. Hall. 1999. Rho GTPases control polarity, protrusion, and adhesion during cell movement. J Cell Biol 144:1235-1244. 
222. Nobes, C. D., and A. Hall. 1995. Rho, rac, and cdc42 GTPases regulate the assembly of multimolecular focal complexes associated with actin stress fibers, lamellipodia, and filopodia. Cell 81:53-62.

223. Ohno-Iwashita, Y., Y. Shimada, A. A. Waheed, M. Hayashi, M. Inomata, M. Nakamura, M. Maruya, and S. Iwashita. 2004. Perfringolysin O, a cholesterol-binding cytolysin, as a probe for lipid rafts. Anaerobe 10:125-134.

224. Olson, J. C., E. M. McGuffie, and D. W. Frank. 1997. Effects of differential expression of the 49-kilodalton exoenzyme $S$ by Pseudomonas aeruginosa on cultured eukaryotic cells. Infect Immun 65:248-256.

225. Olson, M. F., and E. Sahai. 2009. The actin cytoskeleton in cancer cell motility. Clin Exp Metastasis 26:273-287.

226. Ottmann, C., L. Yasmin, M. Weyand, J. L. Veesenmeyer, M. H. Diaz, R. H. Palmer, M. S. Francis, A. R. Hauser, A. Wittinghofer, and B. Hallberg. 2007. Phosphorylationindependent interaction between 14-3-3 and exoenzyme $S$ : from structure to pathogenesis. EMBO J 26:902-913.

227. Pankova, K., D. Rosel, M. Novotny, and J. Brabek. 2010. The molecular mechanisms of transition between mesenchymal and amoeboid invasiveness in tumor cells. Cell Mol Life Sci 67:63-71.

228. Passador, L., J. M. Cook, M. J. Gambello, L. Rust, and B. H. Iglewski. 1993. Expression of Pseudomonas aeruginosa virulence genes requires cell-to-cell communication. Science 260:1127-1130.

229. Pastor, A., J. Chabert, M. Louwagie, J. Garin, and I. Attree. 2005. PscF is a major component of the Pseudomonas aeruginosa type III secretion needle. FEMS Microbiol Lett 253:95-101.

230. Pavlovskis, O. R., and F. B. Gordon. 1972. Pseudomonas aeruginosa exotoxin: effect on cell cultures. J Infect Dis 125:631-636. 
231. Pavlovskis, O. R., and B. Wretlind. 1979. Assessment of protease (elastase) as a Pseudomonas aeruginosa virulence factor in experimental mouse burn infection. Infect Immun 24:181-187.

232. Pederson, K. J., R. Krall, M. J. Riese, and J. T. Barbieri. 2002. Intracellular localization modulates targeting of ExoS, a type III cytotoxin, to eukaryotic signalling proteins. Mol Microbiol 46:1381-1390.

233. Pederson, K. J., A. J. Vallis, K. Aktories, D. W. Frank, and J. T. Barbieri. 1999. The amino-terminal domain of Pseudomonas aeruginosa ExoS disrupts actin filaments via small-molecular-weight GTP-binding proteins. Mol Microbiol 32:393-401.

234. Pelish, H. E., W. Ciesla, N. Tanaka, K. Reddy, M. D. Shair, T. Kirchhausen, and W. I. Lencer. 2006. The Cdc42 inhibitor secramine B prevents cAMP-induced K+ conductance in intestinal epithelial cells. Biochem Pharmacol. 71:1720-1726.

235. Pereira, S. H., M. P. Cervante, S. Bentzmann, and M. C. Plotkowski. 1997. Pseudomonas aeruginosa entry into Caco-2 cells is enhanced in repairing wounded monolayers. Microb Pathog 23:249-255.

236. Pettersson, J., R. Nordfelth, E. Dubinina, T. Bergman, M. Gustafsson, K. E. Magnusson, and H. Wolf-Watz. 1996. Modulation of virulence factor expression by pathogen target cell contact. Science 273:1231-1233.

237. Pfeltz, R. F., J. L. Schmidt, and B. J. Wilkinson. 2001. A microdilution plating method for population analysis of antibiotic-resistant staphylococci. Microb Drug Resist. 7:289295.

238. Picking, W. L., H. Nishioka, P. D. Hearn, M. A. Baxter, A. T. Harrington, A. Blocker, and W. D. Picking. 2005. IpaD of Shigella flexneri is independently required for regulation of Ipa protein secretion and efficient insertion of IpaB and IpaC into host membranes. Infect Immun 73:1432-1440. 
239. Pinna, A., D. Usai, L. A. Sechi, P. Molicotti, S. Zanetti, and A. Carta. 2008. Detection of virulence factors in Pseudomonas aeruginosa strains isolated from contact lensassociated corneal ulcers. Cornea 27:320-326.

240. Plano, G. V. 2010. Coordinating production and distribution: the Pseudomonas aeruginosa ExsACDE regulatory cascade. J Bacteriol 192:1477-1478.

241. Ple, S., V. Job, A. Dessen, and I. Attree. 2010. Cochaperone interactions in export of the type III needle component PscF of Pseudomonas aeruginosa. J Bacteriol 192:38013808.

242. Plotkowski, M. C., M. Chevillard, D. Pierrot, D. Altemayer, J. M. Zahm, G. Colliot, and E. Puchelle. 1991. Differential adhesion of Pseudomonas aeruginosa to human respiratory epithelial cells in primary culture. J Clin Invest 87:2018-2028.

243. Plotkowski, M. C., S. de Bentzmann, S. H. Pereira, J. M. Zahm, O. Bajolet-Laudinat, P. Roger, and E. Puchelle. 1999. Pseudomonas aeruginosa internalization by human epithelial respiratory cells depends on cell differentiation, polarity, and junctional complex integrity. Am J Respir Cell Mol Biol 20:880-890.

244. Plotkowski, M. C., A. M. Saliba, S. H. Pereira, M. P. Cervante, and O. BajoletLaudinat. 1994. Pseudomonas aeruginosa selective adherence to and entry into human endothelial cells. Infect Immun 62:5456-5463.

245. Plotkowski, M. C., J. M. Zahm, J. M. Tournier, and E. Puchelle. 1992. Pseudomonas aeruginosa adhesion to normal and injured respiratory mucosa. Mem Inst Oswaldo Cruz 87 Suppl 5:61-68.

246. Poujade, M., E. Grasland-Mongrain, A. Hertzog, J. Jouanneau, P. Chavrier, B. Ladoux, A. Buguin, and P. Silberzan. 2007. Collective migration of an epithelial monolayer in response to a model wound. Proc Natl Acad Sci U S A 104:15988-15993. 
247. Prahalad, P., I. Calvo, H. Waechter, J. B. Matthews, A. Zuk, and K. S. Matlin. 2004. Regulation of MDCK cell-substratum adhesion by RhoA and myosin light chain kinase after ATP depletion. Am J Physiol Cell Physiol 286:C693-707.

248. Quinaud, M., J. Chabert, E. Faudry, E. Neumann, D. Lemaire, A. Pastor, S. Elsen, A. Dessen, and I. Attree. 2005. The PscE-PscF-PscG complex controls type III secretion needle biogenesis in Pseudomonas aeruginosa. J Biol Chem 280:36293-36300.

249. Radke, J., K. J. Pederson, and J. T. Barbieri. 1999. Pseudomonas aeruginosa exoenzyme $S$ is a biglutamic acid ADP-ribosyltransferase. Infect Immun 67:1508-1510.

250. Ramachandran, R., R. K. Tweten, and A. E. Johnson. 2004. Membrane-dependent conformational changes initiate cholesterol-dependent cytolysin oligomerization and intersubunit beta-strand alignment. Nat Struct Mol Biol. 11:697-705.

251. Ramphal, R., M. T. McNiece, and F. M. Polack. 1981. Adherence of Pseudomonas aeruginosa to the injured cornea: a step in the pathogenesis of corneal infections. Ann Ophthalmol 13:421-425.

252. Ravaoarinoro, M., S. Mohapatra, J. Shore, S. Rawal, A. Omri, J. Yaghi, and F. Oriol. 1996. Serotyping clinical isolates of Pseudomonas aeruginosa in relation to infection site antibiotic susceptibility and beta-lactamase production. Int J Antimicrob Agents 7:65-68.

253. Rello, J., M. Rue, P. Jubert, G. Muses, R. Sonora, J. Valles, and M. S. Niederman. 1997. Survival in patients with nosocomial pneumonia: impact of the severity of illness and the etiologic agent. Crit Care Med 25:1862-1867.

254. Richards, M. J., J. R. Edwards, D. H. Culver, and R. P. Gaynes. 1999. Nosocomial infections in medical intensive care units in the United States. National Nosocomial Infections Surveillance System. Crit Care Med 27:887-892.

255. Richards, M. J., J. R. Edwards, D. H. Culver, and R. P. Gaynes. 1999. Nosocomial infections in pediatric intensive care units in the United States. National Nosocomial Infections Surveillance System. Pediatrics 103:e39. 
256. Ridley, A. J., M. A. Schwartz, K. Burridge, R. A. Firtel, M. H. Ginsberg, G. Borisy, J. T. Parsons, and A. R. Horwitz. 2003. Cell migration: integrating signals from front to back. Science 302:1704-1709.

257. Riese, M. J., U. M. Goehring, M. E. Ehrmantraut, J. Moss, J. T. Barbieri, K. Aktories, and G. Schmidt. 2002. Auto-ADP-ribosylation of Pseudomonas aeruginosa ExoS. J Biol Chem 277:12082-12088.

258. Rietsch, A., I. Vallet-Gely, S. L. Dove, and J. J. Mekalanos. 2005. ExsE, a secreted regulator of type III secretion genes in Pseudomonas aeruginosa. Proc Natl Acad Sci U S A 102:8006-8011.

259. Riff, J. D., J. W. Callahan, and P. M. Sherman. 2005. Cholesterol-enriched membrane microdomains are required for inducing host cell cytoskeleton rearrangements in response to attaching-effacing Escherichia coli. Infect. Immun. 73:7113-7125.

260. Robertson, D. M., W. M. Petroll, J. V. Jester, and H. D. Cavanagh. 2007. Current concepts: contact lens related Pseudomonas keratitis. Cont Lens Anterior Eye 30:94107.

261. Robertson, D. M., W. M. Petroll, J. V. Jester, and H. D. Cavanagh. 2007. The role of contact lens type, oxygen transmission, and care-related solutions in mediating epithelial homeostasis and pseudomonas binding to corneal cells: an overview. Eye Contact Lens 33:394-398; discussion 399-400.

262. Rocchetta, H. L., L. L. Burrows, and J. S. Lam. 1999. Genetics of O-antigen biosynthesis in Pseudomonas aeruginosa. Microbiol Mol Biol Rev 63:523-553.

263. Rocha, C. L., J. Coburn, E. A. Rucks, and J. C. Olson. 2003. Characterization of Pseudomonas aeruginosa exoenzyme $S$ as a bifunctional enzyme in J774A.1 macrophages. Infect Immun 71:5296-5305. 
264. Rocha, C. L., E. A. Rucks, D. M. Vincent, and J. C. Olson. 2005. Examination of the coordinate effects of Pseudomonas aeruginosa ExoS on Rac1. Infect Immun 73:54585467.

265. Romling, U., J. Wingender, H. Muller, and B. Tummler. 1994. A major Pseudomonas aeruginosa clone common to patients and aquatic habitats. Appl Environ Microbiol 60:1734-1738.

266. Ronald, A. 2003. The etiology of urinary tract infection: traditional and emerging pathogens. Dis Mon 49:71-82.

267. Rose, J. J., J. F. Foley, L. Yi, G. Herren, and S. Venkatesan. 2008. Cholesterol is obligatory for polarization and chemotaxis but not for endocytosis and associated signaling from chemoattractant receptors in human neutrophils. J Biomed Sci 15:441461.

268. Rosen, P., and D. S. Misfeldt. 1980. Cell density determines epithelial migration in culture. Proc Natl Acad Sci U S A 77:4760-4763.

269. Roy-Burman, A., R. H. Savel, S. Racine, B. L. Swanson, N. S. Revadigar, J. Fujimoto, T. Sawa, D. W. Frank, and J. P. Wiener-Kronish. 2001. Type III protein secretion is associated with death in lower respiratory and systemic Pseudomonas aeruginosa infections. J Infect Dis 183:1767-1774.

270. Rucks, E. A., J. E. Fraylick, L. M. Brandt, T. S. Vincent, and J. C. Olson. 2003. Cell line differences in bacterially translocated ExoS ADP-ribosyltransferase substrate specificity. Microbiology 149:319-331.

271. Rucks, E. A., and J. C. Olson. 2005. Characterization of an ExoS Type III translocation-resistant cell line. Infect Immun 73:638-643.

272. Rumbaugh, K. P., A. N. Hamood, and J. A. Griswold. 1999. Analysis of Pseudomonas aeruginosa clinical isolates for possible variations within the virulence genes exotoxin $A$ and exoenzyme S. J Surg Res 82:95-105. 
273. Sahai, E., and C. J. Marshall. 2003. Differing modes of tumour cell invasion have distinct requirements for Rho/ROCK signalling and extracellular proteolysis. Nat Cell Biol 5:711-719.

274. Saiman, L., F. Mehar, W. W. Niu, H. C. Neu, K. J. Shaw, G. Miller, and A. Prince. 1996. Antibiotic susceptibility of multiply resistant Pseudomonas aeruginosa isolated from patients with cystic fibrosis, including candidates for transplantation. Clin Infect Dis 23:532-537.

275. Sander, E. E., J. P. ten Klooster, S. van Delft, R. A. van der Kammen, and J. G. Collard. 1999. Rac downregulates Rho activity: reciprocal balance between both GTPases determines cellular morphology and migratory behavior. J Cell Biol 147:10091022.

276. Sanz-Moreno, V., G. Gadea, J. Ahn, H. Paterson, P. Marra, S. Pinner, E. Sahai, and C. J. Marshall. 2008. Rac activation and inactivation control plasticity of tumor cell movement. Cell 135:510-523.

277. Sanz-Moreno, V., and C. J. Marshall. 2009. Rho-GTPase signaling drives melanoma cell plasticity. Cell Cycle 8:1484-1487.

278. Sato, H., J. B. Feix, C. J. Hillard, and D. W. Frank. 2005. Characterization of phospholipase activity of the Pseudomonas aeruginosa type III cytotoxin, ExoU. J Bacteriol 187:1192-1195.

279. Sato, H., and D. W. Frank. 2011. Multi-Functional Characteristics of the Pseudomonas aeruginosa Type III Needle-Tip Protein, PcrV; Comparison to Orthologs in other Gramnegative Bacteria. Front Microbiol 2:142.

280. Sato, H., M. L. Hunt, J. J. Weiner, A. T. Hansen, and D. W. Frank. 2011. Modified needle-tip PcrV proteins reveal distinct phenotypes relevant to the control of type III secretion and intoxication by Pseudomonas aeruginosa. PLoS One 6:e18356. 
281. Sato, H., K. Okinaga, and H. Saito. 1988. Role of pili in the pathogenesis of Pseudomonas aeruginosa burn infection. Microbiol Immunol 32:131-139.

282. Sawa, T., T. L. Yahr, M. Ohara, K. Kurahashi, M. A. Gropper, J. P. Wiener-Kronish, and D. W. Frank. 1999. Active and passive immunization with the Pseudomonas V antigen protects against type III intoxication and lung injury. Nat Med 5:392-398.

283. Schoehn, G., A. M. Di Guilmi, D. Lemaire, I. Attree, W. Weissenhorn, and A. Dessen. 2003. Oligomerization of type III secretion proteins PopB and PopD precedes pore formation in Pseudomonas. Embo J 22:4957-4967.

284. Schraidt, O., and T. C. Marlovits. 2011. Three-dimensional model of Salmonella's needle complex at subnanometer resolution. Science 331:1192-1195.

285. Schulert, G. S., H. Feltman, S. D. Rabin, C. G. Martin, S. E. Battle, J. Rello, and A. R. Hauser. 2003. Secretion of the toxin ExoU is a marker for highly virulent Pseudomonas aeruginosa isolates obtained from patients with hospital-acquired pneumonia. J Infect Dis 188:1695-1706.

286. Schuster, M., C. P. Lostroh, T. Ogi, and E. P. Greenberg. 2003. Identification, timing, and signal specificity of Pseudomonas aeruginosa quorum-controlled genes: a transcriptome analysis. J Bacteriol 185:2066-2079.

287. Schweizer, H. P. 1991. Escherichia-Pseudomonas shuttle vector derived from pUC18/19. Gene 97:109-112.

288. Sekiya, K., M. Ohishi, T. Ogino, K. Tamano, C. Sasakawa, and A. Abe. 2001. Supermolecular structure of the enteropathogenic Escherichia coli type III secretion system and its direct interaction with the EspA-sheath-like structure. Proc Natl Acad Sci U S A 98:11638-11643.

289. Servant, G., O. D. Weiner, P. Herzmark, T. Balla, J. W. Dedat, and H. R. Bourne. 2000. Polarization of chemoattractant receptor signaling during neutrophil chemotaxis. Science 287:1037-1040. 
290. Shaver, C. M., and A. R. Hauser. 2004. Relative contributions of Pseudomonas aeruginosa ExoU, ExoS, and ExoT to virulence in the lung. Infect Immun 72:6969-6977.

291. Shepard, L. A., A. P. Heuck, B. D. Hamman, J. Rossjohn, M. W. Parker, K. R. Ryan, A. E. Johnson, and R. K. Tweten. 1998. Identification of a membrane-spanning domain of the thiol-activated pore-forming toxin Clostridium perfringens perfringolysin $\mathrm{O}$ : an alpha-helical to beta-sheet transition identified by fluorescence spectroscopy. Biochemistry 37:14563-14574.

292. Shestakova, E. A., J. Wyckoff, J. Jones, R. H. Singer, and J. Condeelis. 1999. Correlation of beta-actin messenger RNA localization with metastatic potential in rat adenocarcinoma cell lines. Cancer Res 59:1202-1205.

293. Shimada, Y., M. Maruya, S. Iwashita, and Y. Ohno-Iwashita. 2002. The C-terminal domain of perfringolysin $\mathrm{O}$ is an essential cholesterol-binding unit targeting to cholesterol-rich microdomains. Eur J Biochem. 269:6195-6203.

294. Silver, D. R., I. L. Cohen, and P. F. Weinberg. 1992. Recurrent Pseudomonas aeruginosa pneumonia in an intensive care unit. Chest 101:194-198.

295. Slosberg, E. D., Y. Yao, F. Xing, A. Ikui, M. R. Jirousek, and I. B. Weinstein. 2000. The protein kinase C beta-specific inhibitor LY379196 blocks TPA-induced monocytic differentiation of HL60 cells. Mol Carcinog. 27:166-176.

296. Song, Y., S. V. Lynch, J. Flanagan, H. Zhuo, W. Tom, R. H. Dotson, M. S. Baek, A. Rubio-Mills, G. Singh, E. Kipnis, D. Glidden, R. Brown, O. Garcia, and J. P. WienerKronish. 2007. Increased plasminogen activator inhibitor-1 concentrations in bronchoalveolar lavage fluids are associated with increased mortality in a cohort of patients with Pseudomonas aeruginosa. Anesthesiology 106:252-261.

297. Soong, G., D. Parker, M. Magargee, and A. S. Prince. 2008. The type III toxins of Pseudomonas aeruginosa disrupt epithelial barrier function. J Bacteriol 190:2814-2821. 
298. Soscia, C., A. Hachani, A. Bernadac, A. Filloux, and S. Bleves. 2007. Cross talk between type III secretion and flagellar assembly systems in Pseudomonas aeruginosa. J Bacteriol 189:3124-3132.

299. Sponsel, H. T., R. Breckon, W. Hammond, and R. J. Anderson. 1994. Mechanisms of recovery from mechanical injury of renal tubular epithelial cells. Am J Physiol 267:F257264.

300. Spreter, T., C. K. Yip, S. Sanowar, I. Andre, T. G. Kimbrough, M. Vuckovic, R. A. Pfuetzner, W. Deng, A. C. Yu, B. B. Finlay, D. Baker, S. I. Miller, and N. C. Strynadka. 2009. A conserved structural motif mediates formation of the periplasmic rings in the type III secretion system. Nat Struct Mol Biol 16:468-476.

301. Stebbins, C. E., and J. E. Galan. 2001. Maintenance of an unfolded polypeptide by a cognate chaperone in bacterial type III secretion. Nature 414:77-81.

302. Sun, G. W., and Y. H. Gan. 2010. Unraveling type III secretion systems in the highly versatile Burkholderia pseudomallei. Trends Microbiol 18:561-568.

303. Sundin, C., J. Thelaus, J. E. Broms, and A. Forsberg. 2004. Polarisation of type III translocation by Pseudomonas aeruginosa requires PcrG, PcrV and PopN. Microb Pathog 37:313-322.

304. Sundin, C., M. C. Wolfgang, S. Lory, A. Forsberg, and E. Frithz-Lindsten. 2002. Type IV pili are not specifically required for contact dependent translocation of exoenzymes by Pseudomonas aeruginosa. Microb Pathog 33:265-277.

305. Thiery, J. P. 2002. Epithelial-mesenchymal transitions in tumour progression. Nat Rev Cancer 2:442-454.

306. Tielen, P., M. Narten, N. Rosin, I. Biegler, I. Haddad, M. Hogardt, R. Neubauer, M. Schobert, L. Wiehlmann, and D. Jahn. 2011. Genotypic and phenotypic characterization of Pseudomonas aeruginosa isolates from urinary tract infections. Int $\mathrm{J}$ Med Microbiol 301:282-292. 
307. Tonetti, D. A., C. Henning-Chubb, D. T. Yamanishi, and E. E. Huberman. 1994. Protein kinase C-beta is required for macrophage differentiation of human HL-60 leukemia cells. J Biol Chem. 269:23230-23235.

308. Troisfontaines, P., and G. R. Cornelis. 2005. Type III secretion: more systems than you think. Physiology (Bethesda) 20:326-339.

309. Tsukita, S., and S. Yonemura. 1999. Cortical actin organization: lessons from ERM (ezrin/radixin/moesin) proteins. J Biol Chem 274:34507-34510.

310. Tweten, R. K. 2005. Cholesterol-dependent cytolysins, a family of versatile pore-forming toxins. Infect Immun 73:6199-6209.

311. Urbanowski, M. L., E. D. Brutinel, and T. L. Yahr. 2007. Translocation of ExsE into Chinese hamster ovary cells is required for transcriptional induction of the Pseudomonas aeruginosa type III secretion system. Infect Immun 75:4432-4439.

312. Urbanowski, M. L., G. L. Lykken, and T. L. Yahr. 2005. A secreted regulatory protein couples transcription to the secretory activity of the Pseudomonas aeruginosa type III secretion system. Proc Natl Acad Sci U S A 102:9930-9935.

313. Vakulskas, C. A., K. M. Brady, and T. L. Yahr. 2009. Mechanism of transcriptional activation by Pseudomonas aeruginosa ExsA. J Bacteriol 191:6654-6664.

314. Vallis, A. J., V. Finck-Barbancon, T. L. Yahr, and D. W. Frank. 1999. Biological effects of Pseudomonas aeruginosa type III-secreted proteins on $\mathrm{CHO}$ cells. Infect Immun 67:2040-2044.

315. Vallis, A. J., T. L. Yahr, J. T. Barbieri, and D. W. Frank. 1999. Regulation of ExoS production and secretion by Pseudomonas aeruginosa in response to tissue culture conditions. Infect Immun 67:914-920.

316. van Delden, C. 2007. Pseudomonas aeruginosa bloodstream infections: how should we treat them? Int J Antimicrob Agents 30 Suppl 1:S71-75. 
317. Van Delden, C., and B. H. Iglewski. 1998. Cell-to-Cell Signaling and Pseudomonas aeruginosa Infections Emerging Infect. Dis. 4:551-560.

318. van der Goot, F. G., G. Tran van Nhieu, A. Allaoui, P. Sansonetti, and F. Lafont. 2004. Rafts can trigger contact-mediated secretion of bacterial effectors via a lipid-based mechanism. J Biol Chem. 279:47792-47798.

319. Van Gijsegem, F., C. Gough, C. Zischek, E. Niqueux, M. Arlat, S. Genin, P. Barberis, S. German, P. Castello, and C. Boucher. 1995. The hrp gene locus of Pseudomonas solanacearum, which controls the production of a type III secretion system, encodes eight proteins related to components of the bacterial flagellar biogenesis complex. Mol Microbiol 15:1095-1114.

320. Vance, R. E., A. Rietsch, and J. J. Mekalanos. 2005. Role of the type III secreted exoenzymes S, T, and $\mathrm{Y}$ in systemic spread of Pseudomonas aeruginosa PAO1 in vivo. Infect Immun 73:1706-1713.

321. Veenendaal, A. K., J. L. Hodgkinson, L. Schwarzer, D. Stabat, S. F. Zenk, and A. J. Blocker. 2007. The type III secretion system needle tip complex mediates host cell sensing and translocon insertion. Mol Microbiol 63:1719-1730.

322. Veesenmeyer, J. L., A. R. Hauser, T. Lisboa, and J. Rello. 2009. Pseudomonas aeruginosa virulence and therapy: evolving translational strategies. Crit Care Med $37: 1777-1786$.

323. Ventre, I., A. L. Goodman, I. Vallet-Gely, P. Vasseur, C. Soscia, S. Molin, S. Bleves, A. Lazdunski, S. Lory, and A. Filloux. 2006. Multiple sensors control reciprocal expression of Pseudomonas aeruginosa regulatory RNA and virulence genes. Proc Natl Acad Sci U S A 103:171-176.

324. Viboud, G. I., and J. B. Bliska. 2001. A bacterial type III secretion system inhibits actin polymerization to prevent pore formation in host cell membranes. Embo J 20:5373-5382. 
325. Vidal, F., J. Mensa, M. Almela, J. A. Martinez, F. Marco, C. Casals, J. M. Gatell, E. Soriano, and M. T. Jimenez de Anta. 1996. Epidemiology and outcome of Pseudomonas aeruginosa bacteremia, with special emphasis on the influence of antibiotic treatment. Analysis of 189 episodes. Arch Intern Med 156:2121-2126.

326. Vincent, T. S., J. E. Fraylick, E. M. McGuffie, and J. C. Olson. 1999. ADP-ribosylation of oncogenic Ras proteins by pseudomonas aeruginosa exoenzyme $S$ in vivo. Mol Microbiol 32:1054-1064.

327. Visca, P., F. Chiarini, C. Vetriani, A. Mansi, L. Serino, and N. Orsi. 1991. Epidemiological typing of uropathogenic Pseudomonas aeruginosa strains from hospitalized patients. J Hosp Infect 19:153-165.

328. Von Pawel-Rammingen, U., M. V. Telepnev, G. Schmidt, K. Aktories, H. Wolf-Watz, and R. Rosqvist. 2000. GAP activity of the Yersinia YopE cytotoxin specifically targets the Rho pathway: a mechanism for disruption of actin microfilament structure. Mol Microbiol 36:737-748.

329. Wagner, V. E., D. Bushnell, L. Passador, A. I. Brooks, and B. H. Iglewski. 2003. Microarray analysis of Pseudomonas aeruginosa quorum-sensing regulons: effects of growth phase and environment. J Bacteriol 185:2080-2095.

330. Waheed, A. A., Y. Shimada, H. F. Heijnen, M. Nakamura, M. Inomata, M. Hayashi, S. Iwashita, J. W. Slot, and Y. Ohno-Iwashita. 2001. Selective binding of perfringolysin O derivative to cholesterol-rich membrane microdomains (rafts). Proc. Natl. Acad. Sci. USA 98:4926-4931.

331. Waksman, G. 2009. Going round in circles: the structural biology of type III secretion systems. Nat Struct Mol Biol 16:459-460.

332. Wiehlmann, L., G. Wagner, N. Cramer, B. Siebert, P. Gudowius, G. Morales, T. Kohler, C. van Delden, C. Weinel, P. Slickers, and B. Tummler. 2007. Population structure of Pseudomonas aeruginosa. Proc Natl Acad Sci U S A 104:8101-8106. 
333. Wiener-Kronish, J. P., T. Sakuma, I. Kudoh, J. F. Pittet, D. Frank, L. Dobbs, M. L. Vasil, and M. A. Matthay. 1993. Alveolar epithelial injury and pleural empyema in acute P. aeruginosa pneumonia in anesthetized rabbits. J Appl Physiol 75:1661-1669.

334. Williams, B. J., J. Dehnbostel, and T. S. Blackwell. 2010. Pseudomonas aeruginosa: host defence in lung diseases. Respirology 15:1037-1056.

335. Wisplinghoff, H., T. Bischoff, S. M. Tallent, H. Seifert, R. P. Wenzel, and M. B. Edmond. 2004. Nosocomial bloodstream infections in US hospitals: analysis of 24,179 cases from a prospective nationwide surveillance study. Clin Infect Dis 39:309-317.

336. Woestyn, S., A. Allaoui, P. Wattiau, and G. R. Cornelis. 1994. YscN, the putative energizer of the Yersinia Yop secretion machinery. J Bacteriol 176:1561-1569.

337. Wolf, A. A., Y. Fujinaga, and W. I. Lencer. 2002. Uncoupling of the cholera toxinG(M1) ganglioside receptor complex from endocytosis, retrograde Golgi trafficking, and downstream signal transduction by depletion of membrane cholesterol. J Biol Chem. 277:16249-16256.

338. Wolf, K., I. Mazo, H. Leung, K. Engelke, U. H. von Andrian, E. I. Deryugina, A. Y. Strongin, E. B. Brocker, and P. Friedl. 2003. Compensation mechanism in tumor cell migration: mesenchymal-amoeboid transition after blocking of pericellular proteolysis. J Cell Biol 160:267-277.

339. Wurtele, M., E. Wolf, K. J. Pederson, G. Buchwald, M. R. Ahmadian, J. T. Barbieri, and A. Wittinghofer. 2001. How the Pseudomonas aeruginosa ExoS toxin downregulates Rac. Nat Struct Biol 8:23-26.

340. Xu, B., H. Pelish, T. Kirchhausen, and G. B. Hammond. 2006. Large scale synthesis of the Cdc42 inhibitor secramine A and its inhibition of cell spreading. Org Biomol Chem. 4:4149-4157. 
341. Yahr, T. L., J. T. Barbieri, and D. W. Frank. 1996. Genetic relationship between the 53and 49-kilodalton forms of exoenzyme $S$ from Pseudomonas aeruginosa. J Bacteriol 178:1412-1419.

342. Yahr, T. L., and D. W. Frank. 1994. Transcriptional organization of the trans-regulatory locus which controls exoenzyme S synthesis in Pseudomonas aeruginosa. J Bacteriol 176:3832-3838.

343. Yahr, T. L., J. Goranson, and D. W. Frank. 1996. Exoenzyme S of Pseudomonas aeruginosa is secreted by a type III pathway. Mol Microbiol 22:991-1003.

344. Yahr, T. L., and E. P. Greenberg. 2004. The genetic basis for the commitment to chronic versus acute infection in Pseudomonas aeruginosa. Mol Cell 16:497-498.

345. Yahr, T. L., A. K. Hovey, S. M. Kulich, and D. W. Frank. 1995. Transcriptional analysis of the Pseudomonas aeruginosa exoenzyme S structural gene. J Bacteriol 177:11691178.

346. Yahr, T. L., L. M. Mende-Mueller, M. B. Friese, and D. W. Frank. 1997. Identification of type III secreted products of the Pseudomonas aeruginosa exoenzyme S regulon. J Bacteriol 179:7165-7168.

347. Yahr, T. L., A. J. Vallis, M. K. Hancock, J. T. Barbieri, and D. W. Frank. 1998. ExoY, an adenylate cyclase secreted by the Pseudomonas aeruginosa type III system. Proc Natl Acad Sci U S A 95:13899-13904.

348. Yahr, T. L., and M. C. Wolfgang. 2006. Transcriptional regulation of the Pseudomonas aeruginosa type III secretion system. Mol Microbiol 62:631-640.

349. Yoshida, K., and T. Soldati. 2006. Dissection of amoeboid movement into two mechanically distinct modes. J Cell Sci 119:3833-3844.

350. Zegans, M. E., H. I. Becker, J. Budzik, and G. O'Toole. 2002. The role of bacterial biofilms in ocular infections. DNA Cell Biol 21:415-420. 
351. Zhang, Y., and J. T. Barbieri. 2005. A leucine-rich motif targets Pseudomonas aeruginosa ExoS within mammalian cells. Infect Immun 73:7938-7945.

352. Zhang, Y., Q. Deng, and J. T. Barbieri. 2007. Intracellular localization of type IIIdelivered Pseudomonas ExoS with endosome vesicles. J Biol Chem 282:13022-13032.

353. Zhang, Y., Q. Deng, J. A. Porath, C. L. Williams, K. J. Pederson-Gulrud, and J. T. Barbieri. 2007. Plasma membrane localization affects the RhoGAP specificity of Pseudomonas ExoS. Cell Microbiol 9:2192-2201.

354. Zhang, Y., G. Romanov, and J. B. Bliska. 2011. Type III Secretion System-Dependent Translocation of Ectopically Expressed Yop Effectors into Macrophages by Intracellular Yersinia pseudotuberculosis. Infect Immun 79:4322-4331.

355. Zheng, X., R. Ravatn, Y. Lin, W. C. Shih, A. Rabson, R. Strair, E. Huberman, A. Conney, and K. V. Chin. 2002. Gene expression of TPA induced differentiation in HL60 cells by DNA microarray analysis. Nucleic Acids Res. 30:4489-4499.

356. Zhuo, H., K. Yang, S. V. Lynch, R. H. Dotson, D. V. Glidden, G. Singh, W. R. Webb, B. M. Elicker, O. Garcia, R. Brown, Y. Sawa, B. Misset, and J. P. Wiener-Kronish. 2008. Increased mortality of ventilated patients with endotracheal Pseudomonas aeruginosa without clinical signs of infection. Crit Care Med 36:2495-2503. 
Appendix: Bacterial biofilm diversity in contact lens related infections:

Emerging role of Achromobacter, Stenotrophomonas, and Delftia

The contents of this Appendix have been submitted to:

Investigative Ophthalmology and Visual Science and are currently under review

The authors on the manuscript are: Lee Wiley, Dacie R. Bridge, Lee A. Wiley,

J. Vernon Odom, Thomas Elliott, and Joan C. Olson

Lee Wiley and Dacie R. Bridge are co-first authors 


\section{ABSTRACT}

Purpose: Multi-species biofilms associated with contact lens cases and lenses can predispose individuals to corneal infections. This study utilized culture-independent methods to assess the relationship between the severity of contact lens related keratitis and bacteria residing in lens case and lens biofilms.

Methods: Contact lens cases and lenses from 28 patients referred to the West Virginia University Eye Institute and diagnosed as having mild keratitis, keratitis with focal infiltrates, or corneal ulcers were processed and evaluated for bacterial composition based on $16 \mathrm{~S}$ ribosomal RNA gene sequencing. Cases and lenses from nine asymptomatic contact lens wearers were processed in a similar manner as controls. Relationships between disease severity, bacterial types, and bacterial diversity were statistically evaluated.

Results: Disease severity and presenting visual acuity were found to correlate with an increase in the diversity of bacterial types isolated from contact lens cases. A significant difference was also observed between the number of bacterial types and the three clinical groups. Achromobacter, Stenotrophomonas, and Delftia were identified as the predominant bacteria associated with contact lens related infections among our patients, and scanning electron microscopy revealed that Achromobacter and Stenotrophomonas formed a biofilm on the surface of contact lenses.

Conclusion: Culture independent methods found that bacterial diversity in biofilms isolated from cases and lenses of patients with contact lens related infections increased with severity of disease. Achromobacter, Stenotrophomonas, and Delftia were predominant bacteria identified in this study drawing attention to their emerging role in contact lens related keratitis. 


\section{INTRODUCTION}

Contact lens wear is a major predisposing factor to microbial keratitis and infectious corneal ulcers (4). Microbes can reside on lenses and within lens cases, often in association with multispecies biofilms that serve as reservoirs for the establishment of eye infections $(3,17,23)$. From $24-81 \%$ of contact lens storage cases are contaminated with microbial biofilms with the frequency of contamination increasing in wearers suffering from microbial keratitis (reviewed in (25)). Contamination of contact lenses is less frequent and associated with fewer organisms than lens cases but correlates more closely with organisms that cause corneal infections $(6,15$, 17). Eye infections can be initiated by contact lens induced microabrasions, pathophysiological alterations, or toxic microbial products that compromise the epithelial surface allowing contaminating microbes to adhere and invade (26). Despite improvements in contact lens materials, solutions and wearing habits, epidemiological studies find the incidence of corneal infections has not changed among contact lens wearers in the last two decades $(14,24)$, indicating that microbes have adapted to clinical modifications designed to interfere with corneal infections.

Effective treatment of contact lens related keratitis and corneal ulcers is dependent on the identification of the infectious or non-infectious origin of the problem. Culture dependent methodologies identified Pseudomonas aeruginosa as the most common pathogen in contact lens related infections, followed by Serratia marcenscens, Staphylococcus aureus, Acanthamoeba spp., and Fusarium spp. $(2,9,14)$. Inaccurate characterization and treatment of the offending pathogen may result in prolonged infection, permanent damage to ocular tissues, decreased vision, and in extreme cases removal of the infected tissue (29). A major difficulty in identifying the origin of contact lens infections is that the contaminating organism is often not cultivable $(12,13,21)$. As a result, there is reduced reliance on culturing in the diagnosis of contact lens infections (16), and the current recommended treatment for infections lacking a 
confirmed microbial diagnosis is antibiotics that target $P$. aeruginosa (Bacterial Keratitis PPP, 2008, American Academy of Ophthalmology). Consequently the etiology of the majority contact lens related infections remains unknown.

The goal of this study was to use culture-independent, 16S ribosomal RNA (rRNA) gene analysis to assess bacterial populations associated with contact lens related infections in individuals referred to the West Virginia University Eye Institute with anterior segment disease. We found disease severity and presenting visual acuity to correlate with an increase in the diversity of bacterial types isolated from contact lens cases. Interestingly, the bacterial genera identified most often in our analyses were Achromobacter, Stenotrophomonas, and Delftia, whereas Pseudomonas was rarely identified. Achromobacter, Stenotrophomonas, and Delftia exhibit similar physiological properties as Pseudomonas but have a different antibiotic resistance pattern, which might contribute to their potential emerging role in establishing biofilms associated with contact lens related keratitis. 


\section{MATERIAL AND METHODS}

Subject populations and evaluations. Soft contact lenses and cases were obtained from 28 patients referred to the West Virginia University Eye Institute for contact lens related anterior segment disease. Patients were examined by a single clinician and assigned to one of three categories: 1) mild diffuse keratitis, defined as the presence of stromal inflammatory cells without an area of dense infiltration or epithelial defect; 2) keratitis with focal infiltrates, defined by the presence of stromal inflammatory cells and areas of dense accumulations of cells presenting as a focal opacity; or 3) corneal ulcer, defined as a large epithelial defect with associated suppurative infiltrate and severe generalized anterior segment inflammation. Contact lenses and cases obtained from nine asymptomatic soft contact lens wearers served as controls. Cases and lenses were frozen and maintained at $-20^{\circ} \mathrm{C}$ until the time of analysis. All procedures were performed in accordance with the guidelines of the Declaration of Helsinki using Institutional Review Board approved protocols and informed consent was obtained from each subject.

DNA extraction and 16S rRNA gene sequence analysis. Biofilms were released from contact lens cases by scraping and sonication, and then combined with solutions within cases and subjected to DNA extraction and purification procedures using the Microbial DNA Isolation Kit (Mo Bio Laboratories, Inc., Carlsbad, CA). DNA was extracted and purified from contact lenses by directly placing lenses in tubes provided by the Microbial DNA Isolation Kit, which were then subjected to the same DNA isolation procedures used for contact case samples. Bacterial $16 \mathrm{~S}$ rRNA genes were PCR amplified from purified DNA using universal bacterial 16S rRNA primers, and products were cloned and sequenced as previously described (18).

$16 S$ rRNA gene sequence analysis. Candidate $16 S$ rRNA gene sequences were scanned for a segment of the original primer sequence (ATCAAACT) near the expected position, and the 
match was confirmed by eye. The initial part of each sequence containing uncalled bases $(\mathrm{N})$ was removed, the distal part (vector sequence) was trimmed at the primer, and the sequence was reversed to standard orientation. Chimeric sequences were screened using ChimeraSlayer (8), and six chimeric sequences were identified and removed. Sequences were classified by BLASTN using the legacy executable blastall (NCBI) against a local database, as well as by using the NCBI server with the GenBank non-redundant database. Bacterial types were determined based on $>98 \%$ sequence identity between the $16 \mathrm{~S}$ rRNA gene PCR product and reference database. Bacterial classification used custom Python code (Python 2.6), and the heatmap was generated using matplotlib and Python, as previously described (18).

Scanning electron microscopy (SEM). Biofilm formation on contact lenses was examined by SEM on randomly selected contact lenses from patients with mild keratitis or keratitis with focal infiltrates. Lenses were cut in half, and one half was examined by SEM and the other half was processed for $16 \mathrm{~S}$ rRNA gene sequencing, as above. For SEM, lenses were fixed in $3 \%$ glutaraldehyde in Dulbecco's phosphate buffered saline (DPBS; Hyclone, Logan UT) for 1 hr, then resuspended in $50 \%$ glycerol/50\% deionized (DI) water and shipped to Marshall University for analysis. Comparisons were made between post-fixing lenses in $2 \%$ osmium tetraoxide $\left(\mathrm{OsO}_{4}\right.$; Electron Microscopy Sciences, Hatfield, PA) in DI water for 2 hrs or not, but no difference in image quality was evident. All lenses were dehydrated in a graded ethanol series: $50 \%, 70 \%, 85 \%, 90 \%$, and $95 \%$, followed by two changes in $100 \%$ absolute ethanol. Lenses were cut in half again prior to mounting, and the inside (corneal touching) or the outside surface of the lens was oriented upward for imaging. Samples were immediately dried in the vacuum chamber of a sputter coater (Hummer 6.2, Anatech, Ltd, Union City, CA) and coated with $<10$ nm of gold/palladium (Ladd Research, Williston, VT). Lenses were imaged with a JEOL 5310LV Scanning Electron Microscope (Japan Electron Optics Laboratory; Commerce, MI) and digital images were captured with Printerface software (GW products, SOQUELEC Ltd). 
Statistical analysis. Statistical differences between groups were determined using a nonparametric Kruskal-Wallis $\mathrm{H}$ test for overall significance, which was followed by a MannWhitney $U$ test for significance between two groups. Correlations between variables were tested using the Spearman rank-order coefficient (rho) or a Pearson parametric coefficient $(r)$, as indicated. Statistical calculations were performed using Statistica (Statsoft, Tulsa, OK). 


\section{RESULTS}

Patient evaluations. Patients diagnosed with contact lens related corneal infections were divided into three clinical diagnosis groups: 1) mild diffuse keratitis, 2) keratitis with focal infiltrates, or 3) corneal ulcer, as determined through retrospective chart analysis (Table III). Presenting visual acuity was $20 / 20$ in asymptomatic controls and the mild keratitis group, which worsened to counting fingers or hand motion in patients with severe corneal ulcers. A statistically significant difference $(P<0.001)$ in presenting visual acuity was observed when all groups were compared (Fig. 31), with more severe clinical categories being associated with poorer visual acuity (rho $=0.89, P<0.001$ ). The asymptomatic control and mild keratitis groups did not differ significantly in presenting visual acuity, but all other comparisons of visual acuity between groups were significant $(P<0.05)$, clinically validating the groupings. 
Table III. Patient demographic data

\begin{tabular}{|c|c|c|c|c|c|}
\hline \multicolumn{6}{|c|}{ Clinical Group 1: Mild Keratitis } \\
\hline Patient & Age & Sex & $\begin{array}{l}\text { Infected } \\
\text { Eye }\end{array}$ & $\begin{array}{c}\text { Presenting } \\
\text { Visual Acuity }\end{array}$ & Compliance History \\
\hline 2 & 21 & $\mathrm{M}$ & OD & $20 / 20$ & Slept in lenses \\
\hline 7 & 33 & $\mathrm{~F}$ & OS & $20 / 20$ & Mascara brush injury, slept in lenses \\
\hline 25 & 24 & $\mathrm{~F}$ & OS & $20 / 25$ & Lens overuse, chemical irritant exposure \\
\hline 27 & 54 & $\mathrm{~F}$ & OS & $20 / 25$ & Dry eye \\
\hline 47 & 18 & $\mathrm{M}$ & OS & $20 / 25$ & Poor compliance with cleaning solutions \\
\hline \multicolumn{6}{|c|}{ Clinical Group 2: Keratitis with Focal Infiltrates } \\
\hline Patient & Age & Sex & $\begin{array}{l}\text { Infected } \\
\text { Eye }\end{array}$ & $\begin{array}{c}\text { Presenting } \\
\text { Visual Acuity }\end{array}$ & Compliance History \\
\hline 32 & 37 & $\mathrm{M}$ & OU & $20 / 20 \& 20 / 25$ & Unknown \\
\hline 46 & 31 & $\mathrm{~F}$ & OS & $20 / 25$ & Unknown \\
\hline 52 & 22 & $\mathrm{M}$ & OS & $20 / 30$ & Slept in lenses, poor lens hygiene \\
\hline 56 & 23 & $\mathrm{M}$ & OD & $20 / 30$ & Lens overuse \\
\hline 28 & 22 & $\mathrm{~F}$ & OS & $20 / 40$ & Slept in lenses \\
\hline 62 & 23 & $\mathrm{~F}$ & OS & $20 / 40$ & No lens wear 2 wks prior to presentation \\
\hline 24 & 29 & $\mathrm{~F}$ & OD & $20 / 50$ & Slept in lenses \\
\hline 15 & 19 & $\mathrm{~F}$ & OS & $20 / 60$ & Lenses worn, stored 1 yr then worn again \\
\hline \multicolumn{6}{|c|}{ Clinical Group 3: Corneal Ulcer } \\
\hline Patient & Age & Sex & $\begin{array}{c}\text { Infected } \\
\text { Eye }\end{array}$ & $\begin{array}{c}\text { Presenting } \\
\text { Visual Acuity }\end{array}$ & Compliance History \\
\hline 10 & 48 & $\mathrm{~F}$ & OD & $20 / 400$ & Unknown \\
\hline 9 & 19 & $\mathrm{~F}$ & OS & Count Fingers & Unknown \\
\hline 57 & 51 & $\mathrm{~F}$ & OD & Hand Motion & Unknown \\
\hline 73 & 15 & $\mathrm{M}$ & OD & Hand motion & Lens overuse \\
\hline \multicolumn{6}{|c|}{ Asymptomatic Controls } \\
\hline Patient & Age & Sex & $\begin{array}{l}\text { Infected } \\
\text { Eye }\end{array}$ & $\begin{array}{c}\text { Presenting } \\
\text { Visual Acuity }\end{array}$ & Compliance History \\
\hline 26 & 25 & $\mathrm{~F}$ & N/A & $20 / 20$ & Unknown \\
\hline 48 & 26 & $\mathrm{M}$ & N/A & $20 / 20$ & Unknown \\
\hline 55 & 49 & $\mathrm{~F}$ & N/A & $20 / 20$ & Unknown \\
\hline 68 & 27 & $\mathrm{M}$ & $\mathrm{N} / \mathrm{A}$ & $20 / 20$ & Unknown \\
\hline 69 & 27 & $\mathrm{~F}$ & N/A & $20 / 20$ & Unknown \\
\hline 74 & 33 & $\mathrm{~F}$ & N/A & $20 / 20$ & Unknown \\
\hline 75 & 24 & $\mathrm{M}$ & N/A & $20 / 20$ & Unknown \\
\hline 76 & 40 & $\mathrm{~F}$ & N/A & $20 / 20$ & Unknown \\
\hline 77 & 32 & $\mathrm{M}$ & N/A & $20 / 20$ & Unknown \\
\hline
\end{tabular}




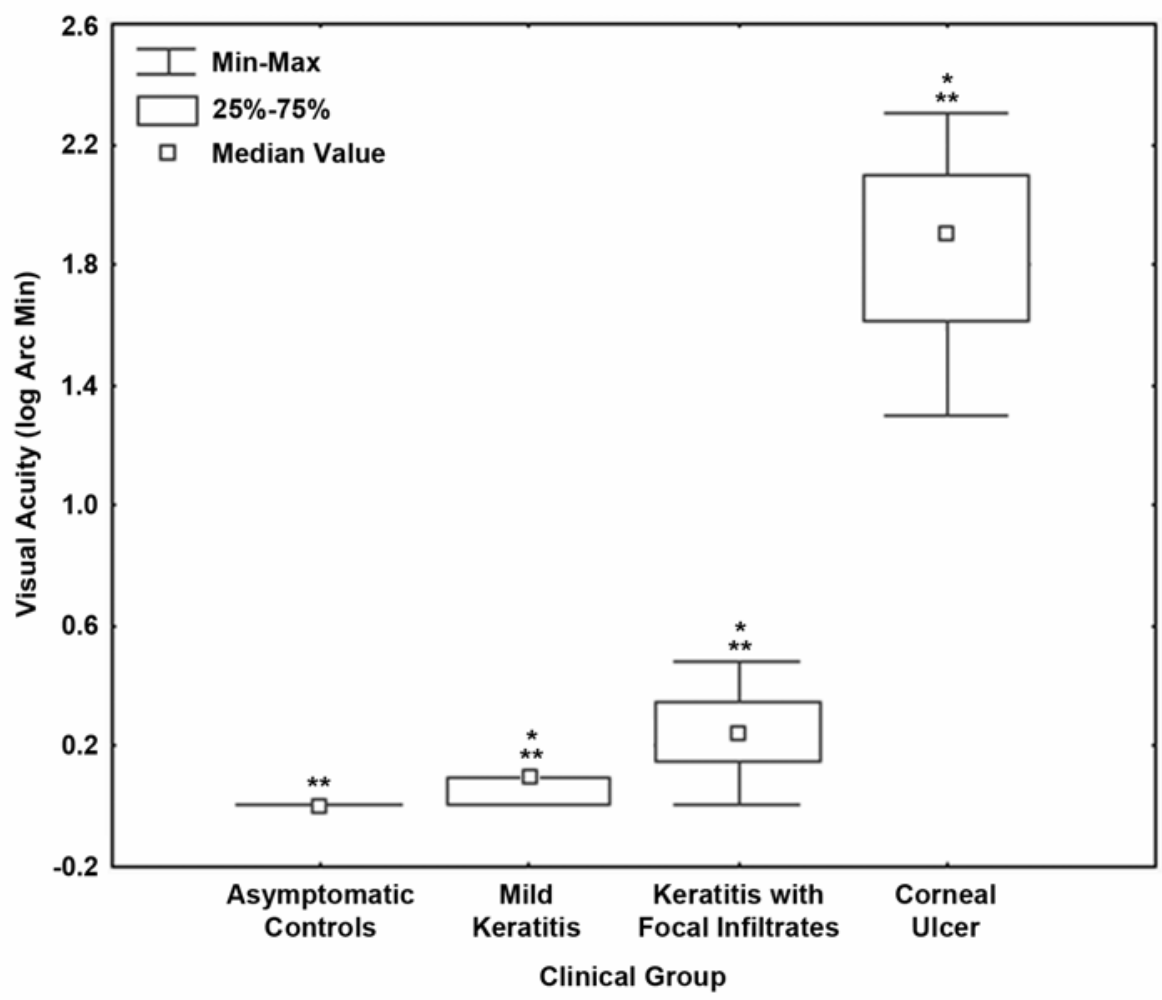

Figure 31. Declining visual acuity is associated with contact lens related corneal disease.

Seventeen patients presenting with contact lens associated corneal infections and having lens cases that produced 16S rRNA gene PCR products, were categorized based on disease severity as having mild keratitis, keratitis with focal infiltrates, or corneal ulcer. Presenting visual acuity was assessed for all patients and nine asymptomatic controls. *Indicates a significant difference $(P<0.05)$ in visual acuity between each of the three clinical groups. ${ }^{* *}$ Indicates a significant difference $(P<0.001)$ in visual acuity when all four groups were compared. 


\section{Relationship between bacterial contamination of contact lens cases and disease severity.}

To assess the poly-bacterial nature of biofilms associated with contact lens related disease, PCR amplification and sequencing of cloned 16 rRNA genes were used to identify bacteria in contact lens cases of our patient group. Of the 28 cases examined, $17(61 \%)$ were positive for a 16S rRNA gene PCR product. The lack of PCR product in the remaining 11 cases is presumed to relate to the absence of PCR amplifiable bacterial DNA in these samples. Contact lens case biofilms and solutions collected in an identical manner from nine asymptomatic contact lens wearers served as controls. Of these samples, one (11\%) produced a $16 \mathrm{~S}$ rRNA PCR product. The increase in PCR amplifiable products from cases of patients with keratitis and corneal ulcers, as compared to control cases, is consistent with bacterial contamination of lens cases being associated with acquisition of contact lens infections.

When the bacterial composition of the 18 PCR positive contact lens case samples (17 disease and one control) was characterized, 38 different bacterial types were identified. The heatmap in Fig. 32 shows the number of clones identified for each bacterial type in each sample and represents a semi-quantitative assessment of the frequency of bacterial types within the case. Variations in the total number of sequences obtained per sample reflect the number of clones subjected to sequencing and to the number of clones that produced readable sequences. When the number of bacterial types identified by $16 \mathrm{~S}$ rRNA gene sequencing was related to disease severity, a significant difference $(P<0.001)$ between the clinical groups was observed (Fig. 33A), with more severe clinical categories associated with an increased number of bacterial types $(r h o=0.82, P<0.001)$. Statistical comparisons of individual clinical groups found the number of bacterial types in the mild keratitis group to be significantly different $(P<0.05)$ from that of the corneal ulcer group. The asymptomatic control group was also found to have fewer bacterial types $(P<0.05)$ than each of the clinical groups. No other statistical differences in bacterial types

were observed among the clinical groups. However, independently of clinical groupings, a 
positive correlation $(r=0.5, P<0.001)$ was observed between increasing numbers of bacterial types and decreased visual acuity (Fig. 33B). 
Figure 32. Identification of bacterial types in cases of patients diagnosed with contact lens related corneal disease. Solutions and biofilms from contact lens cases of 17 patients with corneal infections and one asymptomatic control were evaluated for bacterial composition based on 16S rRNA gene sequencing. Patients were grouped by disease category and further ranked within categories based on worsening visual acuity (determined by increasing log arc min values). Patient reference numbers are indicated at the top of the heatmap. Numbers within colored boxes indicate how many clones of a specific bacterial type were in each sample, and the color of the box reflects bins for observed counts. The total number of clones sequenced for each sample is indicated at the bottom of the heatmap. C refers to control. 


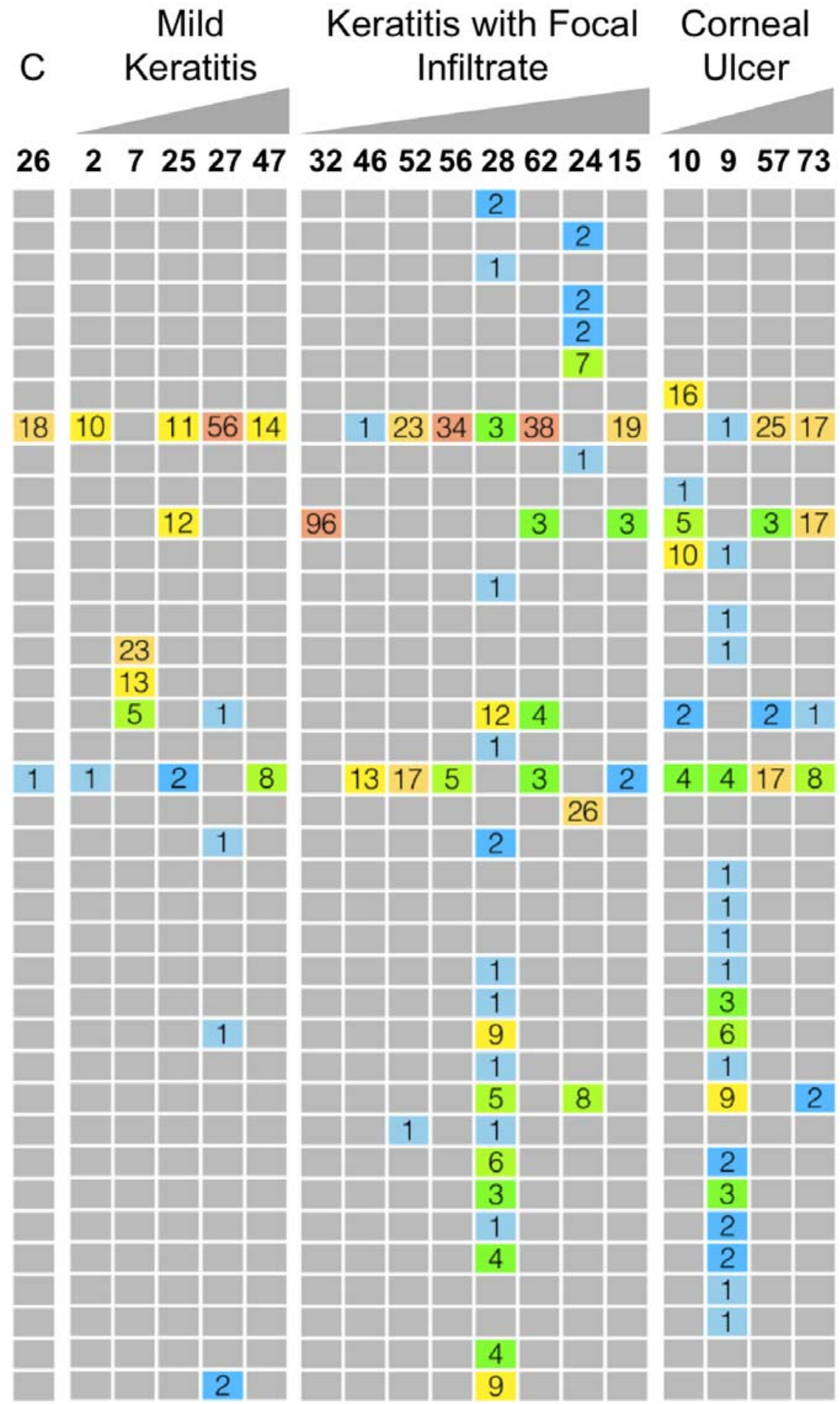

Capnocytophaga

Sphingobacteria

Leptotrichia

Rhizobiales

Devosia

Bradyrhizobium

Ochrobacter

Achromobacter

Burkholderia

Comamonas

Delftia

Oxalobacter

Neisseria

Aeromonas

Pseudomonas

Moraxella

Enterobacteriaceae

Haemophilus

Stenotrophomonas

Xanthomonas

Campylobacter

Corynebacteriaceae

Abiotrophia

Facklamia

Granulicatella

Lactobacillus

Streptococcus

Gemella

Staphylococcus

Eubacterium

Anaerococcus

Finegoldia

Peptoniphilus

Peptostreptococcus

Dialister

Megasphaera

Selenomonas

Veillonella

$191141256122961441396748482438424745 \quad$ Total 
A

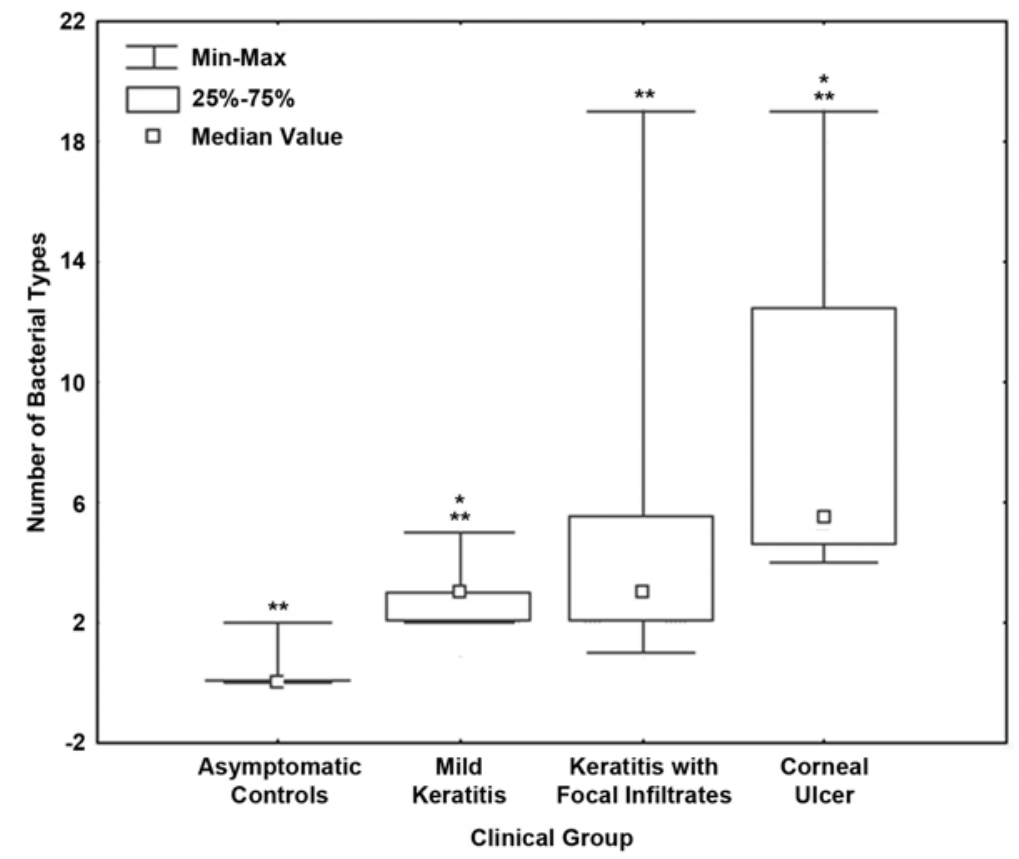

B

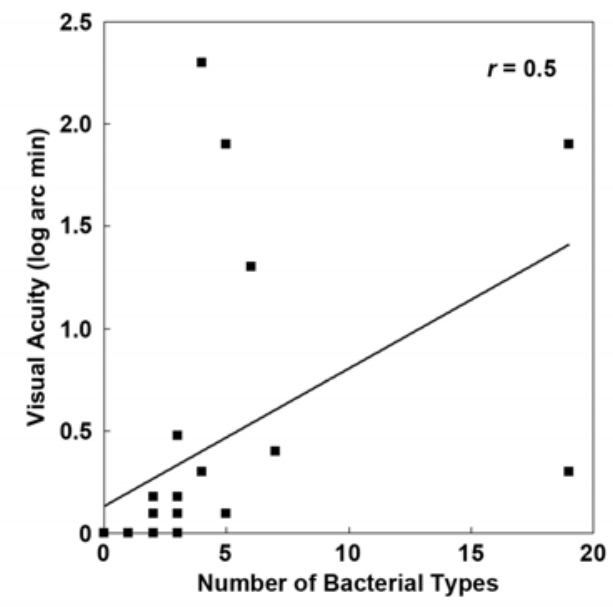

Figure 33. Relationship between bacterial types and severity of disease. A) The number of bacterial types identified by $16 \mathrm{~S}$ rRNA gene sequencing was related to clinical groupings. *Indicates a significant difference $(P<0.05)$ in bacterial types between the mild keratitis group and corneal ulcer group. ${ }^{* *}$ Indicates a significant difference $(P<0.001)$ in bacterial types when all groups were compared. B) Pearson correlation analysis $(r=0.50, P<0.001)$ was used to examine the relationship between visual acuity of individual patients and the number of bacterial types identified by $16 \mathrm{~S}$ rRNA gene sequencing in their contact lens cases. 
When specific bacterial types in contact lens cases were compared, unexpected bacteria emerged as predominant residents. Among the 38 identified bacterial types, Achromobacter had the highest frequency of occurrence $(P<0.05)$ with detection in 13 of $17(76 \%)$ disease associated contact lens cases. Stenotrophomonas was second $(P<0.05)$ with detection in 12 of $17(71 \%)$ disease cases. Delftia and bacteria in the Enterobacteriaceae family were third $(P<0.05)$ with detection in seven of $17(41 \%)$ disease cases. Enterobacteriaceae is a large family of Gram-negative, glucose fermenting bacteria frequently found in the gut, and in our studies included bacteria from the genera Enterobacter, Serratia, Escherichia, Ewingella, and Shigella. Notably, $P$. aeruginosa, recognized to be the most frequent cause of contact lens related eye infections, was detected in only two of 17 cases. Achromobacter and Stenotrophomonas were also detected in the one PCR positive control case at a relative frequency of $18: 1$, respectively, indicating that these bacteria can reside in contact lens cases in the absence of clinical symptoms.

Together these results demonstrate that as corneal infections progress from a mild keratitis to a severe corneal ulcer, the diversity of bacterial types increases and this correlates with a negative impact on visual acuity. In addition, three unexpected bacterial types, Achromobacter, Stenotrophomonas, and Delftia were identified as the predominant contaminating bacteria in contact lens cases of our studies. 
Comparison of bacterial types associated with cases and lenses. To assess the relationship between lens and case contamination in our patient group, we used 16S rRNA gene sequencing to examine bacterial contamination on lenses from 13 of the 17 lens cases analyzed in Fig. 32. When two lenses were provided for analyses, bacterial results for both lenses were combined, and these results are compared with bacteria identified in the cases from the same patient in Table IV. Of the 13 contact lens samples, seven (54\%) were positive for $16 \mathrm{~S}$ rRNA gene PCR products. Lenses from asymptomatic controls were negative for PCR products. Comparisons found bacterial types on lenses to be highly similar with those in the respective case for patients $47,46,62,57$, and 73 , albeit the relative percentage of the bacterial types in lenses and cases varied. The two exceptions to this relationship were: 1) Patient 2, diagnosed with mild keratitis, where bacterial types in the case were limited to Achromobacter (91\%) and Stenotrophomonas (9\%), while multiple diverse types, including Achromobacter and Stenotrophomonas, were detected on lenses; 2) Patient 9, diagnosed with a corneal ulcer, where greater bacterial diversity and a different spectrum of bacteria were detected on the lenses as compared to the case. Interestingly, the spectrum of bacteria on the lenses of patient 9 reflected gut flora, whereas the case bacteria more closely reflected skin or oral flora.

Collectively, comparisons of bacteria associated with lenses and cases of individual patients using $16 \mathrm{~S}$ rRNA gene sequencing found contamination of lenses to be less frequent than cases, as evident by the lack of recovery of PCR products from six of 13 PCR product positive cases, and that the relationship between bacterial types on lenses and cases varied among patients independently of clinical diagnosis. 
Table IV. Bacterial types on lenses in comparison to cases

\begin{tabular}{|c|c|c|c|c|c|}
\hline Patient & Clinical group & Case & \% Bacteria & Lens & $\%$ Bacteria \\
\hline 2 & Mild Keratitis & $\begin{array}{c}\text { Achromobacter } \\
\text { Stenotrophomonas }\end{array}$ & $\begin{array}{c}91(11)^{*} \\
9\end{array}$ & $\begin{array}{c}\text { Achromobacter } \\
\text { Stenotrophomonas } \\
\text { Enterobacteriaceae } \\
\text { Staphylococcus } \\
\text { Xanthomonas } \\
\text { Burkholderia } \\
\text { Eubacterium } \\
\text { Peptostreptococus } \\
\text { Selenomonas } \\
\text { Veillonella }\end{array}$ & $\begin{array}{c}34(32) \\
22 \\
19 \\
6 \\
3 \\
3 \\
3 \\
3 \\
3 \\
3\end{array}$ \\
\hline 47 & Mild keratitis & $\begin{array}{c}\text { Achromobacter } \\
\text { Stenotrophomonas }\end{array}$ & $\begin{array}{c}64(22) \\
36\end{array}$ & $\begin{array}{c}\text { Achromobacter } \\
\text { Stenotrophomonas }\end{array}$ & $\begin{array}{c}57(23) \\
43\end{array}$ \\
\hline 46 & $\begin{array}{l}\text { Keratitis with } \\
\text { focal infiltrate }\end{array}$ & $\begin{array}{c}\text { Stenotrophomonas } \\
\text { Achromobacter }\end{array}$ & $\begin{array}{c}93(14) \\
7 \\
\end{array}$ & $\begin{array}{c}\text { Stenotrophomonas } \\
\text { Achromobacter }\end{array}$ & $\begin{array}{c}94(35) \\
6 \\
\end{array}$ \\
\hline 62 & $\begin{array}{l}\text { Keratitis with } \\
\text { focal infiltrate }\end{array}$ & $\begin{array}{c}\text { Achromobacter } \\
\text { Enterobacteriaceae } \\
\text { Delftia } \\
\text { Stenotrophomonas }\end{array}$ & $\begin{array}{l}79(48) \\
8 \\
6 \\
6 \\
\end{array}$ & $\begin{array}{c}\text { Achromobacter } \\
\text { Enterobacteriaceae } \\
\text { Delftia } \\
\text { Stenotrophomonas }\end{array}$ & $\begin{array}{c}66(94) \\
18 \\
10 \\
6 \\
\end{array}$ \\
\hline 9 & Corneal ulcer & $\begin{array}{l}\text { Staphylococcus } \\
\text { Streptococcus } \\
\text { Stenotrophomonas } \\
\text { Lactobacillus } \\
\text { Finegoldia } \\
\text { Peptoniphilus } \\
\text { Peptostreptococcus } \\
\text { Anaerococcus } \\
\text { Pseudomonas } \\
\text { Dialister } \\
\text { Megasphera } \\
\text { Achromobacter } \\
\text { Aeromonas } \\
\text { Gemella } \\
\text { Abiotrophia } \\
\text { Facklamia } \\
\text { Granulicatella } \\
\text { Corynebacterium } \\
\text { Oxalobacter }\end{array}$ & $\begin{array}{l}21(42) \\
14 \\
10 \\
7 \\
7 \\
5 \\
5 \\
5 \\
2 \\
2 \\
2 \\
2 \\
2 \\
2 \\
2 \\
2 \\
2 \\
2 \\
2\end{array}$ & $\begin{array}{c}\text { Enterobacteriaceae } \\
\text { Veillonella } \\
\text { Selenomonas } \\
\text { Campylobacter } \\
\text { Dialister } \\
\text { Stenotrophomonas } \\
\text { Peptostreptococcus } \\
\text { Xanthomonas } \\
\text { Rhizobiales } \\
\text { Leptotrichia }\end{array}$ & $\begin{array}{l}35(23) \\
22 \\
9 \\
9 \\
9 \\
4 \\
4 \\
4 \\
4 \\
4\end{array}$ \\
\hline 57 & Corneal ulcer & $\begin{array}{c}\text { Achromobacter } \\
\text { Stenotrophomonas } \\
\text { Delftia } \\
\text { Enterobacteriaceae }\end{array}$ & $\begin{array}{c}53(47) \\
36 \\
6 \\
4\end{array}$ & $\begin{array}{c}\text { Stenotrophomonas } \\
\text { Achromobacter } \\
\text { Delftia } \\
\text { Enterobacteriaceae }\end{array}$ & $\begin{array}{c}56^{\dagger}(48) \\
35 \\
6 \\
2\end{array}$ \\
\hline 73 & Corneal ulcer & $\begin{array}{c}\text { Achromobacter } \\
\text { Delftia } \\
\text { Stenotrophomonas } \\
\text { Staphylococcus } \\
\text { Enterobacteriaceae }\end{array}$ & $\begin{array}{l}38(45) \\
38 \\
18 \\
4 \\
2\end{array}$ & $\begin{array}{c}\text { Delftia } \\
\text { Stenotrophomonas } \\
\text { Achromobacter }\end{array}$ & $\begin{array}{c}38(42) \\
36 \\
26\end{array}$ \\
\hline
\end{tabular}

* Parentheses enclose number of sequences analyzed per sample.

${ }^{\dagger}$ Only left lens was analyzed. 
SEM analysis of contact lenses. To assess if bacteria identified by $16 \mathrm{~S}$ rRNA gene sequencing exist within a biofilm, two lenses were randomly selected and cut in half, and one half was analyzed by $16 \mathrm{~S}$ rRNA gene sequencing while the other half was processed for SEM analysis. 16S rRNA gene sequence analysis identified Achromobacter (57\%) and Stenotrophomonas $(43 \%)$ on the lenses of patient 47 , diagnosed with mild keratitis. Achromobacter and Stenotrophomonas are both Gram-negative rods, and SEM analysis revealed the presence of a dense film of rod-shaped bacteria on both the inside and outside surface of the lens from patient 47 (Fig. 34A). 16S rRNA gene sequence analysis of the lenses of patient 46 also identified Achromobacter (6\%) and Stenotrophomonas (94\%), and SEM analysis revealed a biofilm containing rod-shaped bacteria (Fig. 34B). These results confirm that Achromobacter and Stenotrophomonas are able to form biofilms on the surface of contact lenses of patients diagnosed with keratitis. 
Figure 34. Bacterial biofilm formation on lenses from patients with contact lens related corneal disease. To assess that ability of bacterial populations identified by 16S rRNA gene analyses to form a biofilm on contact lenses, randomly selected contact lenses from: A) patient 47, diagnosed with mild keratitis, or B) patient 46 , diagnosed with keratitis with focal infiltrates were analyzed by scanning electron microscopy, and this was related to bacterial types identified on lenses. Lenses were cut in half to analyze both the inside (corneal touching) and outside surfaces. The right panel shows a higher magnification of the area enclosed in the white square in the left panel. White arrows point to bacteria. Scale bars represent 1 or $5 \mu \mathrm{m}$ for low magnification and 0.5 or $1 \mu \mathrm{m}$ for higher magnification. 
A

Inside Lens Surface

Outside Lens Surface

B

Inside Lens Surface

Outside Lens Surface

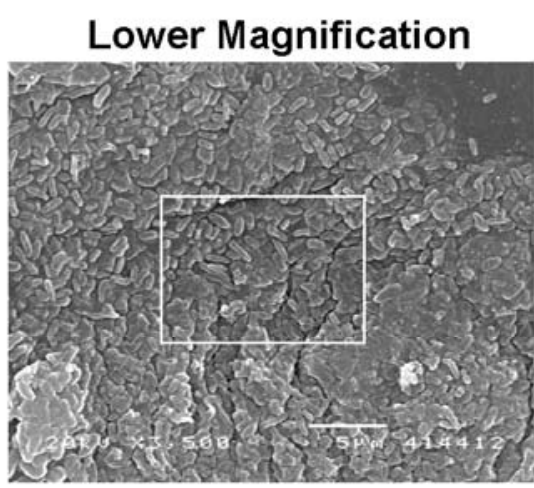

Mild Keratitis (Patient 47)

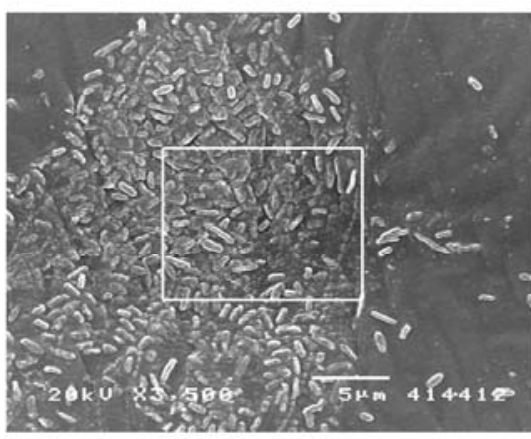

Higher Magnification
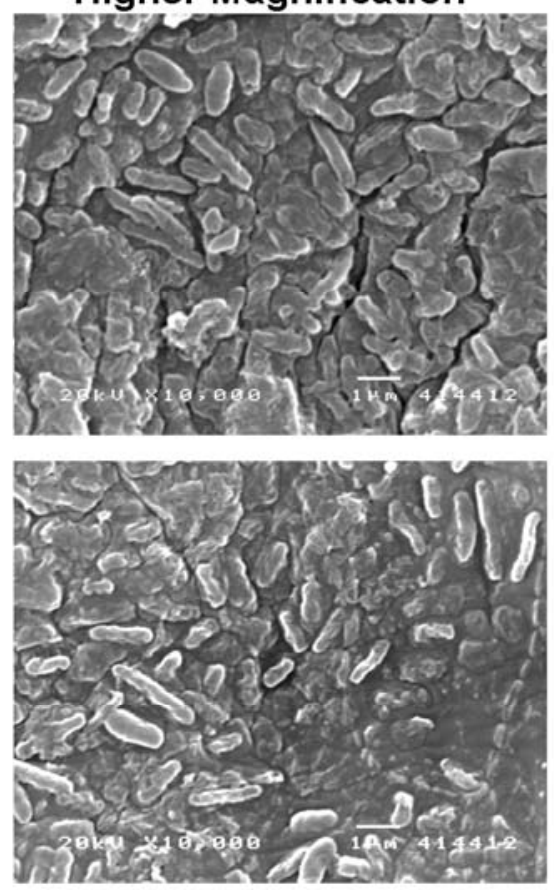

Keratitis with Focal Infiltrate (Patient 46)

Lower Magnification

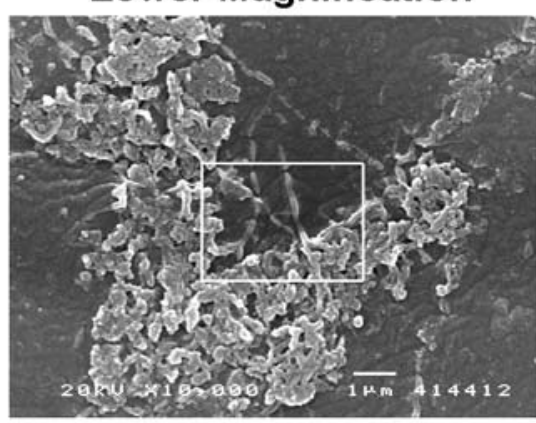

Higher Magnification
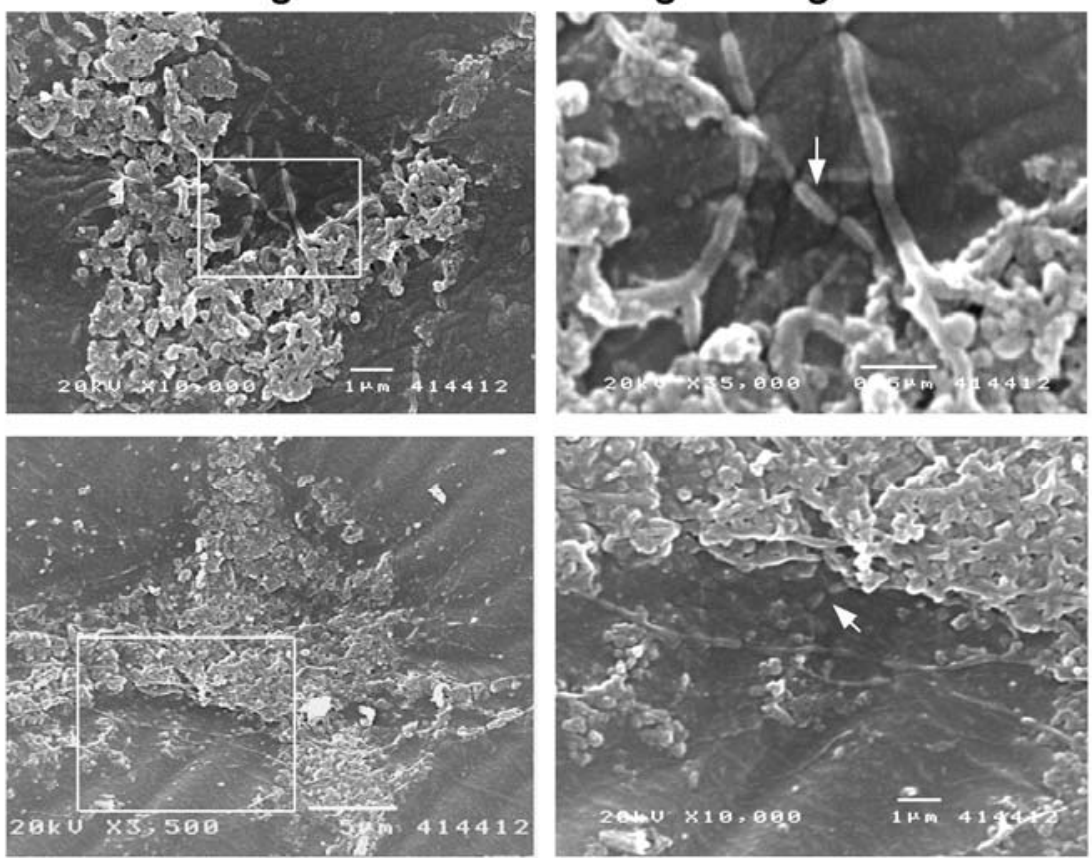


\section{DISCUSSION}

This study used 16S rRNA gene sequencing to examine bacterial populations present in cases and on lenses of patients diagnosed with contact lens related corneal disease. 16S rRNA gene analysis was chosen for this study to enhance the likelihood of characterizing the bacterial diversity in biofilms associated with specific disease states. Patients were categorized based on disease severity as having mild keratitis, keratitis with focal infiltrates, or corneal ulcers, and we observed a significant decrease in presenting visual acuity and a significant increase in bacterial diversity as the severity of corneal disease increased.

Of the patients in this study, a 16S rRNA gene PCR product was obtained from 17 of 28 cases (61\%). The lower than anticipated recovery of PCR products can be related to several factors. First it is possible, particularly in milder cases of keratitis, that the disease might be caused by noninfectious agents or toxic products, and hence no bacterial DNA would be present. Second, sources other than contact lenses, such as saliva, tap water, or a fingertip, might have introduced the offending microbe into the eye. Third, it is possible that disease might have resulted from fungi, amoebae, or a viral sources, which are recognized to contaminate from 4$59 \%$ of contact lens cases (9), but would not be detected by our analysis due to the specificity of the PCR amplification primers for bacterial 16S rRNA genes.

Interestingly, when the $16 \mathrm{~S}$ rRNA gene products from the 17 PCR positive samples were cloned and sequenced, a diverse and unexpected panel of bacteria was identified. A total of 38 different bacterial types were identified in lens cases, which was significantly higher than that obtained from asymptomatic controls. In most patients the prevalence and diversity of Gramnegative bacteria increased with disease severity, but in contrast with previous studies (reviewed in $(9,14))$, relatively few Gram-negative Pseudomonas were detected in our samples. Gram-positive bacterial diversity was evident in cases of two patients, 28 and 9, who presented 
with a clinical diagnosis of keratitis with focal infiltrates and corneal ulcer, respectively. The similar Gram-positive diversity but different clinical diagnosis of these two patients may be explained by their time of presentation in the Eye Institute in relation to their stage of disease, and patient 28 might progress to develop a corneal ulcer in the absence of intervention. In general, bacterial contamination on lenses was detected less frequently than within cases in our study, which is consistent with previous bacterial culturing studies $(9,17,27)$. However, in five of seven comparisons highly similar bacterial types were identified on lenses and cases, which is consistent with the premise that lens cases serve as a source of contaminating bacteria leading to eye infections. Attempts were made during our studies to confirm that bacteria in cases and lenses were also present in corneal scrapings of infected eyes, but we were unable to obtain PCR amplifiable 16S rRNA gene products from these scrapings to allow analysis of bacterial composition.

The prevalence of Achromobacter and Stenotrophomonas in cases and lenses in our study was unexpected. Both bacteria are Gram-negative, aerobic, non-glucose fermenting rods, which can be confused clinically with Pseudomonas $(11,22)$. Like Pseudomonas, Achromobacter, and Stenotrophomonas are opportunistic pathogens, but differ from Pseudomonas in being less virulent and having different antibiotic resistance profiles. Achromobacter is considered an emerging pathogen and has been found as a contaminant in solutions, including disinfectant solutions $(1,10)$. A limited number of Achromobacter corneal infections have been reported and are characterized as slow progressing, recurrent infections, associated with localized infiltration and resistance to aminoglycosides, first generation cephalosporins, and variable susceptibility to fluoroquinolones (19). Notably, these are the antibiotics included in the regimen for treatment of eye infections that lack an identified causative organism (Bacterial Keratitis PPP, 2008, American Academy of Ophthalmology). The number of reported Stenotrophomonas eye infections is also limited, and notably the major predisposing factor to Stenotrophomonas 
infections is prior exposure to antibiotics (22). Stenotrophomonas is often a co-infecting organism and has limited invasiveness, high antibiotic resistance, but unlike Achromobacter is resistant to carbapenems and can develop additional resistance during an infection (5). The third most frequent contaminant of lens cases in our studies was Delftia, also a Gram-negative, non-glucose fermenting opportunistic pathogen resistant to aminoglycosides and $\beta$-lactam antibiotics. A recent study in Japan identified Gram-negative, non-glucose fermenting bacteria as the most frequent contaminants of contact lens cases in a student population (7). In these studies, Stenotrophomonas was identified as the most frequent contaminant, followed by Delftia, Pseudomonas, and then Achromobacter. Similarly, a study comparing bacterial contamination of commercially available contact lens solutions found the rate of bacterial contamination to vary with different solutions, but again the most prevalent contaminating bacteria were Delftia, Stenotrophomonas, and Achromobacter (28). It should also be noted that the one asymptomatic control case in our study that produced a PCR amplifiable 16S rRNA gene product was contaminated with Achromobacter and Stenotrophomonas.

While contamination is required for an infection, it must be accompanied by some form of corneal compromise for infection to be established. The most common compromising factor for contact lens corneal infections is overnight lens wear (20). Of the 17 patients in our study, seven were documented as having slept in their lenses or having lens overuse. A second threat to corneal infections is biofilm formation, which provides bacterial populations with resistance to antiseptics and antibiotics (3). Interestingly, Gram-negative, non-fermenting bacteria, such as Pseudomonas, Achromobacter, Stenotrophomonas, and Delftia have a propensity to form biofilms $(7,25)$. The frequent association of Achromobacter, Stenotrophomonas, and Delftia within contact lens cases of patients in our study can thus be explained by their ability to contaminate contact lens solutions, their resistance to antibiotics empirically used to treat eye infections, and their ability to form biofilms, as was visualized for Achromobacter and 
Stenotrophomonas in SEM analysis. In turn, the reason that these bacteria have the tendency to remain less of a threat than $P$. aeruginosa in eye infections is that they lack the arsenal of virulence factors that make Pseudomonas infections particularly problematic.

In summary, difficulties in culturing bacteria from eye infections have led to less reliance on the identification of causative organism in the treatment of eye disease. Instead, empiric administration of antibiotics that target the most prevalent cause of severe eye infections, $P$. aeruginosa, is recommended and has proven to be both clinically successful and economic. In this study when 16S rRNA gene sequencing was used to examine bacterial populations in cases and lenses of patients with contact lens related disease, diverse (generally nonPseudomonas) bacteria were identified, and the presence and severity of disease increased in association with the complexity of the biofilm. Three clinically rare Gram-negative bacteria, Achromobacter, Stenotrophomonas, and Delftia, emerged as frequent inhabitants of cases and lenses of patients in our study. These bacteria maintain similar biochemical and metabolic properties of $P$. aeruginosa, can survive in contact lens solutions, and may form the basis for biofilms linked to the establishment of eye infections that are resistant to antibiotics used to treat Pseudomonas. 


\section{ACKNOWLEDGEMENTS}

We would like to acknowledge the clinical assistance of Drs. Michael McAllister and Scott Jamerson in our studies, and the technical assistance of LaKeisha Hall in the development of 16S rRNA gene analysis procedures. SEM analysis was performed by Michael Norton, David Neff, and Francesca Karle from Marshall University Department of Chemistry in conjunction with the Marshall University Molecular and Biological Imaging Center. 


\section{REFERENCES}

1. Ahmed, M. S., C. Nistal, R. Jayan, M. Kuduvalli, and H. K. Anijeet. 2009. Achromobacter xylosoxidans, an emerging pathogen in catheter-related infection in dialysis population causing prosthetic valve endocarditis: a case report and review of literature. Clin Nephrol 71:350-354.

2. Alfonso, E., S. Mandelbaum, M. J. Fox, and R. K. Forster. 1986. Ulcerative keratitis associated with contact lens wear. Am J Ophthalmol 101:429-433.

3. Behlau, I., and M. S. Gilmore. 2008. Microbial biofilms in ophthalmology and infectious disease. Arch Ophthalmol 126:1572-1581.

4. Bourcier, T., F. Thomas, V. Borderie, C. Chaumeil, and L. Laroche. 2003. Bacterial keratitis: predisposing factors, clinical and microbiological review of 300 cases. $\mathrm{Br} \mathrm{J}$ Ophthalmol 87:834-838.

5. Chen, Y. F., P. C. Chung, and C. H. Hsiao. 2005. Stenotrophomonas maltophilia keratitis and scleritis. Chang Gung Med J 28:142-150.

6. Das, S., H. Sheorey, H. R. Taylor, and R. B. Vajpayee. 2007. Association between cultures of contact lens and corneal scraping in contact lens related microbial keratitis. Arch Ophthalmol 125:1182-1185.

7. Furuhata, K., N. Ishizaki, Y. Kawakami, and M. Fukuyama. 2010. Bacterial contamination of stock solutions in storage cases for contact lens, and the disinfectantresistance of isolates. Biocontrol Sci 15:81-85.

8. Haas, B. J., D. Gevers, A. M. Earl, M. Feldgarden, D. V. Ward, G. Giannoukos, D. Ciulla, D. Tabbaa, S. K. Highlander, E. Sodergren, B. Methe, T. Z. DeSantis, J. F. Petrosino, R. Knight, and B. W. Birren. 2011. Chimeric 16S rRNA sequence formation and detection in Sanger and 454-pyrosequenced PCR amplicons. Genome Res 21:494504. 
9. Hall, B. J., and L. Jones. 2010. Contact lens cases: the missing link in contact lens safety? Eye Contact Lens 36:101-105.

10. Holmes, B., J. J. Snell, and S. P. Lapage. 1977. Strains of Achromobacter xylosoxidans from clinical material. J Clin Pathol 30:595-601.

11. Igra-Siegman, Y., H. Chmel, and C. Cobbs. 1980. Clinical and laboratory characteristics of Achromobacter xylosoxidans infection. J Clin Microbiol 11:141-145.

12. Knox, C. M., V. Cevellos, and D. Dean. 1998. 16S ribosomal DNA typing for identification of pathogens in patients with bacterial keratitis. J Clin Microbiol 36:34923496.

13. Levey, S. B., H. R. Katz, D. A. Abrams, M. J. Hirschbein, and M. J. Marsh. 1997. The role of cultures in the management of ulcerative keratitis. Cornea 16:383-386.

14. Liesegang, T. J. 1997. Contact lens-related microbial keratitis: Part I: Epidemiology. Cornea 16:125-131.

15. Martins, E. N., M. E. Farah, L. S. Alvarenga, M. C. Yu, and A. L. Hoflin-Lima. 2002. Infectious keratitis: correlation between corneal and contact lens cultures. CLAO J 28:146-148.

16. McDonnell, P. J., J. Nobe, W. J. Gauderman, P. Lee, A. Aiello, and M. Trousdale. 1992. Community care of corneal ulcers. Am J Ophthalmol 114:531-538.

17. McLaughlin-Borlace, L., F. Stapleton, M. Matheson, and J. K. Dart. 1998. Bacterial biofilm on contact lenses and lens storage cases in wearers with microbial keratitis. $J$ Appl Microbiol 84:827-838.

18. Olson, J. C., C. F. Cuff, S. Lukomski, E. Lukomska, Y. Canizales, B. Wu, R. J. Crout, J. G. Thomas, D. W. McNeil, R. J. Weyant, M. L. Marazita, B. J. Paster, and T. Elliott. 2011. Use of $16 \mathrm{~S}$ ribosomal RNA gene analyses to characterize the bacterial signature associated with poor oral health in West Virginia. BMC Oral Health 11:7. 
19. Reddy, A. K., P. Garg, V. Shah, and U. Gopinathan. 2009. Clinical, microbiological profile and treatment outcome of ocular infections caused by Achromobacter xylosoxidans. Cornea 28:1100-1103.

20. Schein, O. D., P. O. Buehler, J. F. Stamler, D. D. Verdier, and J. Katz. 1994. The impact of overnight wear on the risk of contact lens-associated ulcerative keratitis. Arch Ophthalmol 112:186-190.

21. Schein, O. D., R. J. Glynn, E. C. Poggio, J. M. Seddon, and K. R. Kenyon. 1989. The relative risk of ulcerative keratitis among users of daily-wear and extended-wear soft contact lenses. A case-control study. Microbial Keratitis Study Group. N Engl J Med 321:773-778.

22. Senol, E. 2004. Stenotrophomonas maltophilia: the significance and role as a nosocomial pathogen. J Hosp Infect 57:1-7.

23. Stapleton, F., and J. Dart. 1995. Pseudomonas keratitis associated with biofilm formation on a disposable soft contact lens. Br J Ophthalmol 79:864-865.

24. Stapleton, F., L. Keay, I. Jalbert, and N. Cole. 2007. The epidemiology of contact lens related infiltrates. Optom Vis Sci 84:257-272.

25. Szczotka-Flynn, L. B., E. Pearlman, and M. Ghannoum. 2010. Microbial contamination of contact lenses, lens care solutions, and their accessories: a literature review. Eye Contact Lens 36:116-129.

26. Tsubota, K., S. Hata, I. Toda, Y. Yagi, M. Sakata, and J. Shimazaki. 1996. Increase in corneal epithelial cell size with extended wear soft contact lenses depends on continuous wearing time. Br J Ophthalmol 80:144-147.

27. Vermeltfoort, P. B., J. M. Hooymans, H. J. Busscher, and H. C. van der Mei. 2008. Bacterial transmission from lens storage cases to contact lenses-Effects of lens care solutions and silver impregnation of cases. J Biomed Mater Res B Appl Biomater 87:237-243. 
28. Willcox, M. D., N. Carnt, J. Diec, T. Naduvilath, V. Evans, F. Stapleton, S. Iskandar, N. Harmis, P. L. de la Jara, and B. A. Holden. 2010. Contact lens case contamination during daily wear of silicone hydrogels. Optom Vis Sci 87:456-464.

29. Zegans, M. E., H. I. Becker, J. Budzik, and G. O'Toole. 2002. The role of bacterial biofilms in ocular infections. DNA Cell Biol 21:415-420. 
Curriculum Vitae 


\section{Dacie R. Bridge}

Email: $\quad$ dbridge@mix.wvu.edu

Phone: (304) 293-3951

Address: West Virginia University School of Medicine

Dept of Microbiology, Immunology and Cell

Biology

PO Box 9177

Morgantown WV, 26506-9177

\section{EDUCATION}

West Virginia University School of Medicine, Morgantown, West Virginia

Doctorate of Philosophy candidate, Immunology and Microbial Pathogenesis;

Expected December 2, 2011

Thesis: Insight into the Cellular Alterations Required for Establishing Opportunistic

Pseudomonas aeruginosa Infections

Advisor: Joan C. Olson, Ph.D.

Saint Vincent College, Latrobe, Pennsylvania

Bachelor of Science, Biology; May 2005

Thesis: The Role of LytR and LytS, a Two Component Regulatory System, in Cell Autolysis in

Vancomycin Intermediate Resistant Staphylococcus aureus

Advisor: Jennifer Koehl, Ph.D.

Cumulative G.P.A: 3.28/4.0

\section{RESEARCH EXPERIENCE}

Doctoral Research, Laboratory of Joan C. Olson, Ph.D. 2006-Present West Virginia University School of Medicine, Morgantown, West Virginia

Thesis: Insight into the Cellular Alterations Required for Establishing Opportunistic Pseudomonas aeruginosa Infections

My Ph.D. research has two related but independent purposes. First, I wanted to determine how host cells are involved in Pseudomonas aeruginosa type III secretion (T3S). Little is known about host cell involvement in T3S in any bacterial system, and as an opportunistic pathogen, Pseudomonas offered an advantage in studying this problem since cell lines sensitive or resistant to Pseudomonas T3S were identified in our laboratory. My project involved investigating the mechanism underlying eukaryotic cell sensitivity to Pseudomonas T3S. In exploring this problem, using a bacterial-eukaryotic cell co-culture system and multiple approaches involving cell function inhibitors, targeted siRNA and immunofluorescence microscopy, we were able to identify a relationship between eukaryotic cell leading edge properties and sensitivity to Pseudomonas T3S. In subsequent studies it was recognized that the T3S effector, ExoS, which targets leading edge proteins, also influences the efficiency T3S translocation. I used Pseudomonas strains expressing enzymatic mutant forms of ExoS to further dissect the relationship between leading edge properties in MTC, MTLn3, and MDCK cell models, Pseudomonas T3S, and the establishment of Pseudomonas infections.

Second, I wanted to explore a clinical problem, examining the role of Pseudomonas in contact lens associated microbial keratitis and corneal ulcer infections in the West Virginia clinics. Pseudomonas has been implicated as a leading cause of contact lens associated infections. However, $80 \%$ of infections remain without a positive microbiological diagnosis. These studies 
developed in collaboration with Dr. Lee Wiley, an ophthalmologist in the WVU clinics, and in association with our laboratory's use of 16S rRNA gene analysis to identify bacterial profiles in clinical biofilms. Unexpectedly, the predominant bacteria found in contact lens cases and on lenses of individuals with keratitis were Achromobacter and Stenotrophomonas, rather than Pseudomonas. In addition, we found that as contact lens infections progressed to include increased infiltrates, formation of corneal ulcers, and loss of visual acuity, the bacterial profiles became more complex in association with a decreased frequency of Achromobacter. We are currently in the process of finalizing statistical analyses of bacterial profiles in relation to clinical symptoms and preparing this study for publication.

Skills that I have developed in these studies include: eukaryotic and bacterial cell culture, SDSPAGE and Western blot analysis, siRNA transfection, confocal immunofluorescence microscopy utilizing antibodies and Quantum dots, protein purification, DNA isolation and analysis, PCR amplification, and cloning.

Undergraduate Research Thesis, Laboratory of Jennifer Koehl, Ph.D., January 2004-May 2005

Saint Vincent College, Department of Biology, Latrobe, Pennsylvania

Thesis: The Role of LytR and LytS, a Two Component Regulatory System, in Cell Autolysis in Vancomycin Intermediate Resistant Staphylococcus aureus

The purpose of this project was to determine if the LytR/S two component regulatory system was involved in $S$. aureus resistance to vancomycin. My research involved characterizing the LytR/S two component regulatory system in vancomycin intermediate resistant $S$. aureus strains as compared to their vancomycin sensitive parent strain. I performed strain selection, PCR amplification of the LytR/S genes, DNA purification from agarose gels, and RNA extraction and isolation.

Undergraduate research student, Laboratory of Cynthia Walter, Ph.D. Summer 2004

Saint Vincent College, Department of Biology, Latrobe, Pennsylvania

Project: Isolation and Identification of Leptothrix discophora from Local Streams Polluted with Abandoned Mine Drainage

L. discophora grows in slow running water containing iron and manganese as a thick, reddish, flocculent material that forms a film on the surface of the water. These conditions have been observed in streams affected by abandoned mine drainage surrounding Saint Vincent College. The purpose of my project was to design experiments to be performed by a summer undergraduate student that would determine if $L$. discophora was also the cause of the stream conditions in our area. For this project, I researched $L$. discophora and designed experiments to identify this organism, which included stream characterization, collection of samples, microbiological identification, and 16S rRNA gene analysis.

Undergraduate research student, Laboratory of Jennifer Koehl, Ph.D. Summer 2003 Saint Vincent College, Department of Biology, Latrobe, Pennsylvania Project: Reassessment of Physical Characteristics of Vancomycin Intermediate Resistant Staphylococcus aureus (VISA) Strains

The purpose of this project was to characterize vancomycin intermediate resistant $S$. aureus strains acquired from Illinois State University for use in subsequent studies. In this project, I was responsible for characterizing the $S$. aureus strains based on minimum antibiotic inhibitory concentrations to ensure that the strains retained their antibiotic resistance as compared to their vancomycin sensitive parent strains before being utilized for experiments. 


\section{TEACHING EXPERIENCE}

Mentoring: Undergraduate Research Student, Lee Wiley

July 2010-Present

The purpose of the project was to determine the bacterial profiles associated with contact lens associated eye infections. My duties included directing, teaching, and supervising Lee in the isolation, purification, and amplification of 16S rRNA genes from contact lenses and cases of patients diagnosed with microbial keratitis or corneal ulcers. I also reviewed and helped Lee prepare his final paper for his senior capstone project.

Mentoring: West Virginia IDeA Network of Biomedical Research Excellence (INBRE) Summer Rotation Student, Philip Adams

June-July, 2010

The purpose of this project was to further investigate the role of host cell leading edge properties in Pseudomonas aeruginosa type III secretion. My duties included helping Philip with experimental design and teaching him how to culture eukaryotic and bacterial cells, to perform eukaryotic and bacterial cell co-culture studies, and to analyze the results of these studies using SDS-PAGE, Western blotting, and immunofluorescence techniques. Philip was selected to present his project at the West Virginia State Capitol for the annual Undergraduate Research Day and his summer research was highlighted in WVUToday: "WVU, Marshall biomedical research cooperation benefits entire state." Philip also won second place for his project at the 8th Middle Atlantic Undergraduate Research Conference held in Buckhannon, WV.

Mentoring: West Virginia INBRE and Health Sciences and Technology Academy High School Teacher Summer Intern, Wendy Lee

June-August, 2010

The purpose of this project was to design experiments to visualize Pseudomonas aeruginosa type III secretion inside the host cell. My duties included supervising Wendy in experimental design and teaching her eukaryotic cell, bacterial cell and co-culturing techniques and immunofluorescent microscopy. Wendy's research project was highlighted in WVUToday: "WVU, Marshall biomedical research cooperation benefits entire state."

Mentoring: First-year Biomedical Sciences Rotation Student, Kelly Miller July-August 2007

The purpose of this rotation was to investigate the effects of DMSO on Pseudomonas aeruginosa type III secretion. My duties including teaching Kelly eukaryotic cell culture, bacterial-eukaryotic co-culture, SDS-PAGE and Western blotting techniques. I also supervised Kelly with experimental design and results interpretation.

Graduate Teaching Assistant: MICB 323, Medical Microbiology for Medical Technology Students January 12 through February 14, 2009; 30 students

My duties included teaching laboratory lectures, assisting students during laboratory exercises, and grading laboratory reports, quizzes, and laboratory practicals. 
Graduate Teaching Assistant: MICB 322, Medical Microbiology for Medical Technology Students

Spring Semester, 2008; 31 students

My duties included culturing strains for class, teaching laboratory lectures, assisting students during the laboratory exercises, and grading laboratory reports, quizzes, and laboratory practicals.

Graduate Teaching Assistant: MICB 327, Medical Parasitology for Medical Technology Students Spring Semester, 2007; 27 students

My duties included assisting students with identification of organisms and proctoring lab practicals.

\section{AWARDS AND HONORS}

2011 Travel Award Recipient: Student Travel Grant Award to attend and present a poster at the American Society for Microbiology $111^{\text {th }}$ General Meeting in New Orleans, Louisiana

$1^{\text {st }}$ Place Award: Oral Presentations, 2011 E.J. Van Liere Memorial Convocation. West Virginia University, Morgantown WV

Travel Award Recipient: Travel Award from the MidAtlantic Microbial Pathogenesis Meeting to attend and present a poster presentation

2010 Travel Award Recipient: Robert E. Stitzel Travel Award from the West Virginia University Office of Graduate Academic Affairs to attend and present an oral presentation at the American Society for Microbiology Kadner Institute

Travel Award Recipient: West Virginia University Travel Award from the West Virginia University Health Science Center Research and Graduate Education Office to attend and present an oral presentation at the American Society for Microbiology Kadner Institute

$2^{\text {nd }}$ Place Award: Basic Science Poster, 2010 E.J. Van Liere Convocation/Health Science Center Research Day

2009 Grant Recipient: Golden Key Research Grant from the Golden Key International Honour Society

Travel Award Recipient: Robert E. Stitzel Travel Award from the West Virginia University Office of Graduate Academic Affairs to attend and present a poster at the Cold Spring Microbial Pathogenesis and Host Response Meeting

Travel Award Recipient: West Virginia University Travel Award from the West Virginia University Health Science Center Research and Graduate Education Office to attend and present a poster at the Cold Spring Microbial Pathogenesis and Host Response Meeting 
Travel Award Recipient: Cold Spring Harbor Laboratories Student Travel Award to attend and present a poster at the Microbial Pathogenesis and Host Response Meeting, Long Island, New York

$1^{\text {st }}$ Place Award: Basic Science Poster, 2009 E.J. Van Liere Convocation/Health Science Center Research Day

2008 Travel Award Recipient: Corporate Activities Program Student Travel Grant Award to attend and present a poster at the American Society for Microbiology $108^{\text {th }}$ General Meeting in Boston, Massachusetts

2007 West Virginia University Student Travel Award: 1 of 5 travel awards given to attend a scientific meeting from the West Virginia University Office of the Provost

$2^{\text {nd }}$ Place Award: Basic Science Poster, 2007 E.J. Van Liere Convocation/Health Science Center Research Day

2003 Who Among Students in American Colleges and Universities: Saint Vincent College; selected by a college committee

\section{PEER REVIEWED PUBLICATIONS}

Bridge, D.R. ${ }^{1}$, M.J. Novotny ${ }^{1}$, E.R. Moore, and J.C. Olson (2010). Role of Host Cell Polarity and Leading Edge Properties in Pseudomonas Type III Secretion. Microbiology. 156(2):356-373.

${ }^{1}$ Co-first authors.

\section{MANUSCRIPTS UNDER REVIEW}

Bridge, D.R., K.H. Martin, E.R. Moore, W.M. Lee, J.A. Carroll, and J.C. Olson. Gaining Insight into the Opportunistic Nature of Pseudomonas aeruginosa Infections through Manipulations of the T3S Effector ExoS (Submitted and under review at Infection and Immunity).

Wiley, L. ${ }^{1}$, D.R. Bridge ${ }^{1}$, L.A. Wiley, J.V. Odom, T. Elliott, and J.C. Olson. Bacterial biofilm diversity in contact lens related infections: Emerging role of Achromobacter, Stenotrophomonas and Delftia. ${ }^{1}$ Co-first authors (Submitted and under review at Investigative Ophthalmology and Visual Science)

\section{Poster:}

\section{PRESENTATIONS}

Bridge, D.R. K.H. Martin, E.R. Moores, W.M. Lee and J.C. Olson. (2011). How Pseudomonas aeruginosa Type III Secretion Effector ExoS Regulates Translocation into Epithelial Cells. 111 ${ }^{\text {th }}$ General Meeting of the American Society for Microbiology. New Orleans, Louisiana.

Bridge, D.R., K.H. Martin, and J.C. Olson. (2011). Insight into the Cellular Alteration Allowing Susceptibility to Opportunistic Pseudomonas aeruginosa Infections. West Virginia University Van Liere Convocation/ Research Day. Morgantown, West Virginia. 
Bridge, D.R., W.M. Lee, K.H. Martin, and J.C. Olson. (2011). Pseudomonas aeruginosa Type III Secretion Effector ExoS Regulates Translocation into T24 Epithelial Cells. 2011 MidAtlantic Microbial Pathogenesis Meeting, Wintergreen, Virginia.

Adams, P.A., D.R. Bridge, and J.C. Olson. (2011). Polarized and confluent MDCK cell monolayer sensitivity to Pseudomonas aeruginosa type III secretion. 8th Middle Atlantic Undergraduate Research Conference. Buckhannon, West Virginia.

Adams, P.A., D.R. Bridge, and J.C. Olson. (2011). Polarized and confluent MDCK cell monolayer sensitivity to Pseudomonas aeruginosa type III secretion. Undergraduate Research Day at the Capitol. Charleston, West Virginia.

Bridge, D.R., M.J. Novotny, W. Lee, and J.C. Olson. (2010). Host and Bacterial Factors that Influence Pseudomonas Type III Secretion Translocon Function. Gordon Research Conference on Microbial Toxins and Pathogenicity. Waterville Valley, New Hampshire.

Bridge, D.R. and J.C. Olson. (2010). Host and Bacterial Factors that Influence Pseudomonas Type III Secretion Translocon Function. West Virginia University Van Liere Convocation/ Research Day. Morgantown, West Virginia.

Bridge, D.R. and J.C. Olson. (2009). Characterizing the Role of Host Cell Polarity and Leading Edge Properties in Pseudomonas aeruginosa Type III Secretion. Cold Spring Harbor Laboratory Meeting on Microbial Pathogenesis and Host Response. Cold Spring Harbor, New York.

Novotny, M.J., D.R. Bridge, and J.C. Olson (2009). Leading edge associated complexes influence sensitivity to Pseudomonas aeruginosa type III secretion. Cold Spring Harbor Laboratory Meeting on Microbial Pathogenesis and Host Response. Cold Spring Harbor, New York.

Bridge, D.R. and J.C. Olson (2009). Leading Edge Properties and Host Cell Polarity Function in Pseudomonas aeruginosa Type III Secretion. West Virginia University Van Liere Convocation/ Research Day. Morgantown, West Virginia.

Novotny, M.J., D.R. Bridge ${ }_{2}$ and J.C. Olson (2009). The role of the host cell in Pseudomonas type III secretion. West Virginia University Van Liere Convocation/ Research Day. Morgantown, West Virginia.

Bridge, D.R., R.K. Tweten, and J.C. Olson (2008). The Role of Host Cell Adhesion Processes in Pseudomonas aeruginosa Type III Secretion. $108^{\text {th }}$ General Meeting of the American Society for Microbiology. Boston, Massachusetts.

Novotny, M.J., D.R. Bridge, and J.C. Olson (2008). Mechanosensory characteristics of the Pseudomonas aeruginosa type III secretion translocon. $108^{\text {th }}$ General Meeting of the American Society for Microbiology. Boston, Massachusetts.

Bridge, D.R. and J.C. Olson (2008). Membrane Properties Influence Host Cell Susceptibility to Pseudomonas aeruginosa Type III Secretion. West Virginia University Van Liere Convocation/ Research Day. Morgantown, West Virginia. 
Bridge, D.R., J.B. Salazar, and J.C. Olson (2007). The Role of Host Cell Membrane Cholesterol in Type III Secretion of Pseudomonas aeruginosa. West Virginia University Van Liere Convocation/ Research Day. Morgantown, West Virginia.

\section{Oral:}

Bridge, D.R., (2011). Insight into the Cellular Alteration Allowing Susceptibility to Opportunistic Pseudomonas aeruginosa Infections. 2011 Van Liere Memorial Convocation. West Virginia University. Morgantown, West Virginia.

Bridge, D.R. (2010). The Role of Pseudomonas in Contact Lens Related Ocular Infections. American Society for Microbiology Kadner Institute. Michigan State University, East Lansing, Michigan.

\section{CAREER DEVELOPMENT}

American Society for Microbiology Kadner Institute, July 24-27, 2010

Michigan State University, East Lansing, Michigan

Preparing Future Faculty Workshop, May 15, 2010

West Virginia University, Morgantown, West Virginia

\section{UNIVERSITY SERVICE}

2010-2011 Secretary: West Virginia University Health Sciences Center Student Advisory Board

2009-2011 Biomedical Science Representative: West Virginia University Health Sciences Center Student Advisory Board, selected by board and recommended by the Dean of Biomedical Sciences for the position

2009-2010 Secretary: Golden Key International Honour Society, West Virginia University Chapter

\section{PROFESSIONAL SOCIETIES}

2009-Present Golden Key International Honour Society, West Virginia University Chapter 2005-Present Student member: American Society for Microbiology

2004-2005 Student Member: Allegheny Branch of the American Society for Microbiology 
Copyrights and Permissions 


\section{Figure 1:}

ELSEVI ER LI CENSE

TERMS AND CONDITIONS

Nov 15, 2011

This is a License Agreement between Dacie R Bridge ("You") and Elsevier ("Elsevier") provided by Copyright Clearance Center ("CCC"). The license consists of your order details, the terms and conditions provided by Elsevier, and the payment terms and conditions.

All payments must be made in full to CCC. For payment instructions, please see information listed at the bottom of this form.

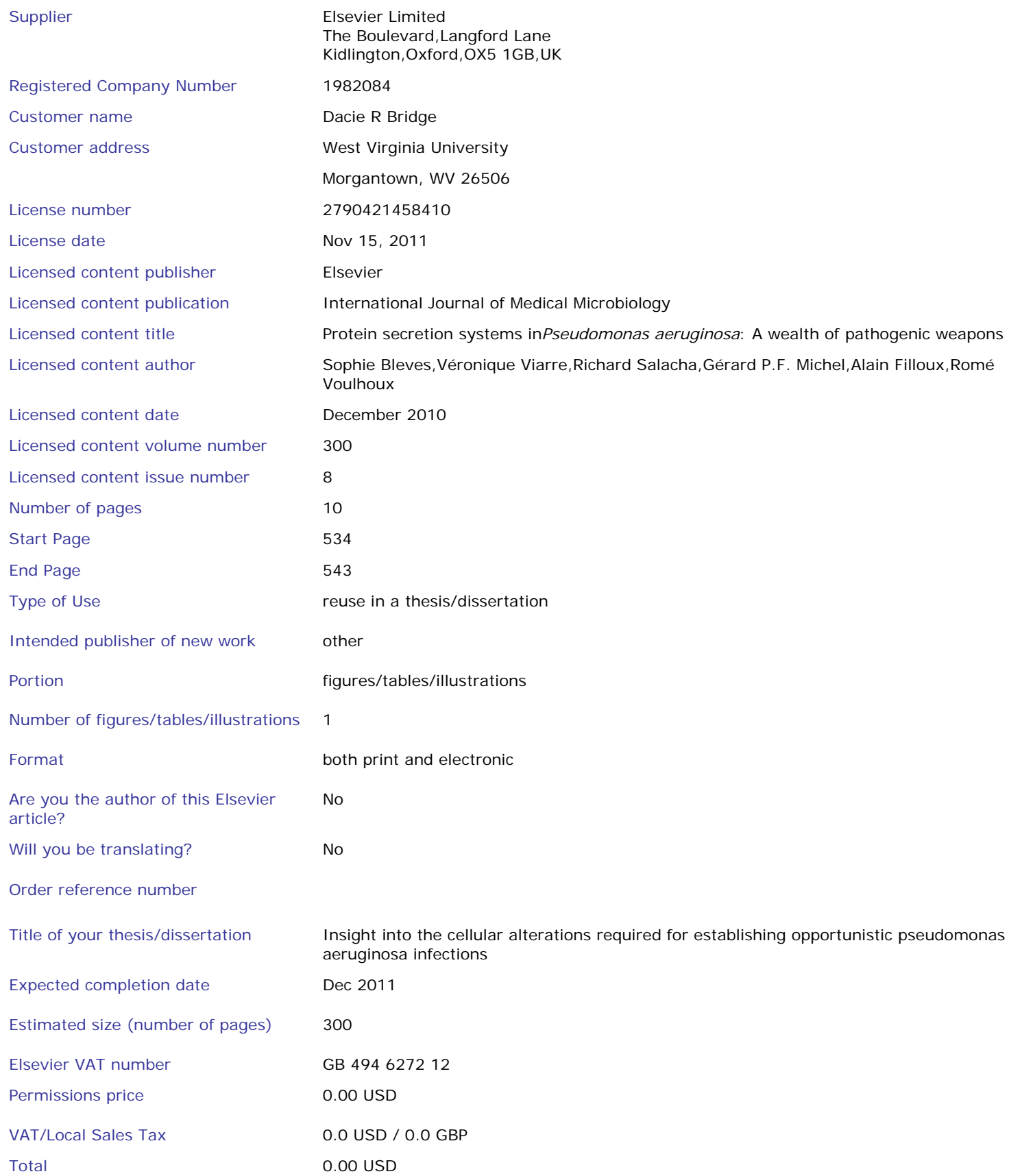


Figures 2 and 5:

NATURE PUBLISHI NG GROUP LI CENSE TERMS AND CONDITIONS

This is a License Agreement between Dacie R Bridge ("You") and Nature Publishing Group ("Nature Publishing Group") provided by Copyright Clearance Center ("CCC"). The license consists of your order details, the terms and conditions provided by Nature Publishing Group, and the payment terms and conditions.

\begin{tabular}{|c|c|}
\hline License Number & 2790430238129 \\
\hline License date & Nov 15, 2011 \\
\hline Licensed content publisher & Nature Publishing Group \\
\hline Licensed content publication & Nature Reviews Microbiology \\
\hline Licensed content title & The type III secretion system of Pseudomonas aeruginosa: infection by injection \\
\hline Licensed content author & Alan R. Hauser \\
\hline Licensed content date & Sep 1, 2009 \\
\hline Type of Use & reuse in a thesis/dissertation \\
\hline Requestor type & academic/educational \\
\hline Format & print and electronic \\
\hline Portion & figures/tables/illustrations \\
\hline Number of figures/tables/illustrations & 2 \\
\hline High-res required & no \\
\hline Figures & Figure 1 and Figure 2 \\
\hline Author of this NPG article & no \\
\hline \multicolumn{2}{|l|}{ Your reference number } \\
\hline Title of your thesis / dissertation & $\begin{array}{l}\text { Insight into the cellular alterations required for establishing opportunistic pseudomonas } \\
\text { aeruginosa infections }\end{array}$ \\
\hline Expected completion date & Dec 2011 \\
\hline Estimated size (number of pages) & 300 \\
\hline Total & 0.00 USD \\
\hline
\end{tabular}




\section{Figure 3:}

AMERI CAN SOCIETY FOR MI CROBI OLOGY LICENSE TERMS AND CONDITIONS

This is a License Agreement between Dacie R Bridge ("You") and American Society for Microbiology ("American Society for Microbiology") provided by Copyright Clearance Center ("CCC"). The license consists of your order details, the terms and conditions provided by American Society for Microbiology, and the payment terms and conditions.

\begin{tabular}{|c|c|}
\hline License Number & 2790421140317 \\
\hline License date & Nov 15, 2011 \\
\hline Licensed content publisher & American Society for Microbiology \\
\hline Licensed content publication & Microbiology and Molecular Biology Reviews \\
\hline Licensed content title & Process of Protein Transport by the Type III Secretion System \\
\hline Licensed content author & Partho Ghosh \\
\hline Licensed content date & Dec 1, 2004 \\
\hline Volume & 68 \\
\hline \multicolumn{2}{|l|}{ Issue } \\
\hline Start page & 771 \\
\hline End page & 795 \\
\hline Type of Use & Dissertation/Thesis \\
\hline Format & Print and electronic \\
\hline Portion & Figures/tables/images \\
\hline Number of figures/tables & 1 \\
\hline \multicolumn{2}{|l|}{ Order reference number } \\
\hline Title of your thesis / dissertation & $\begin{array}{l}\text { Insight into the cellular alterations required for establishing opportunistic pseudomonas } \\
\text { aeruginosa infections }\end{array}$ \\
\hline Expected completion date & Dec 2011 \\
\hline Estimated size(pages) & 300 \\
\hline Billing Type & Invoice \\
\hline Billing address & $\begin{array}{l}\text { West Virginia University } \\
\text { Dept of Microbiology } \\
\text { Morgantown, WV } 26506 \\
\text { United States }\end{array}$ \\
\hline \multicolumn{2}{|l|}{ Customer reference info } \\
\hline Total & 0.00 USD \\
\hline
\end{tabular}


Figure 3 Legend:

AMERI CAN SOCIETY FOR MI CROBI OLOGY LICENSE TERMS AND CONDITIONS

This is a License Agreement between Dacie R Bridge ("You") and American Society for Microbiology ("American Society for Microbiology") provided by Copyright Clearance Center ("CCC"). The license consists of your order details, the terms and conditions provided by American Society for Microbiology, and the payment terms and conditions.

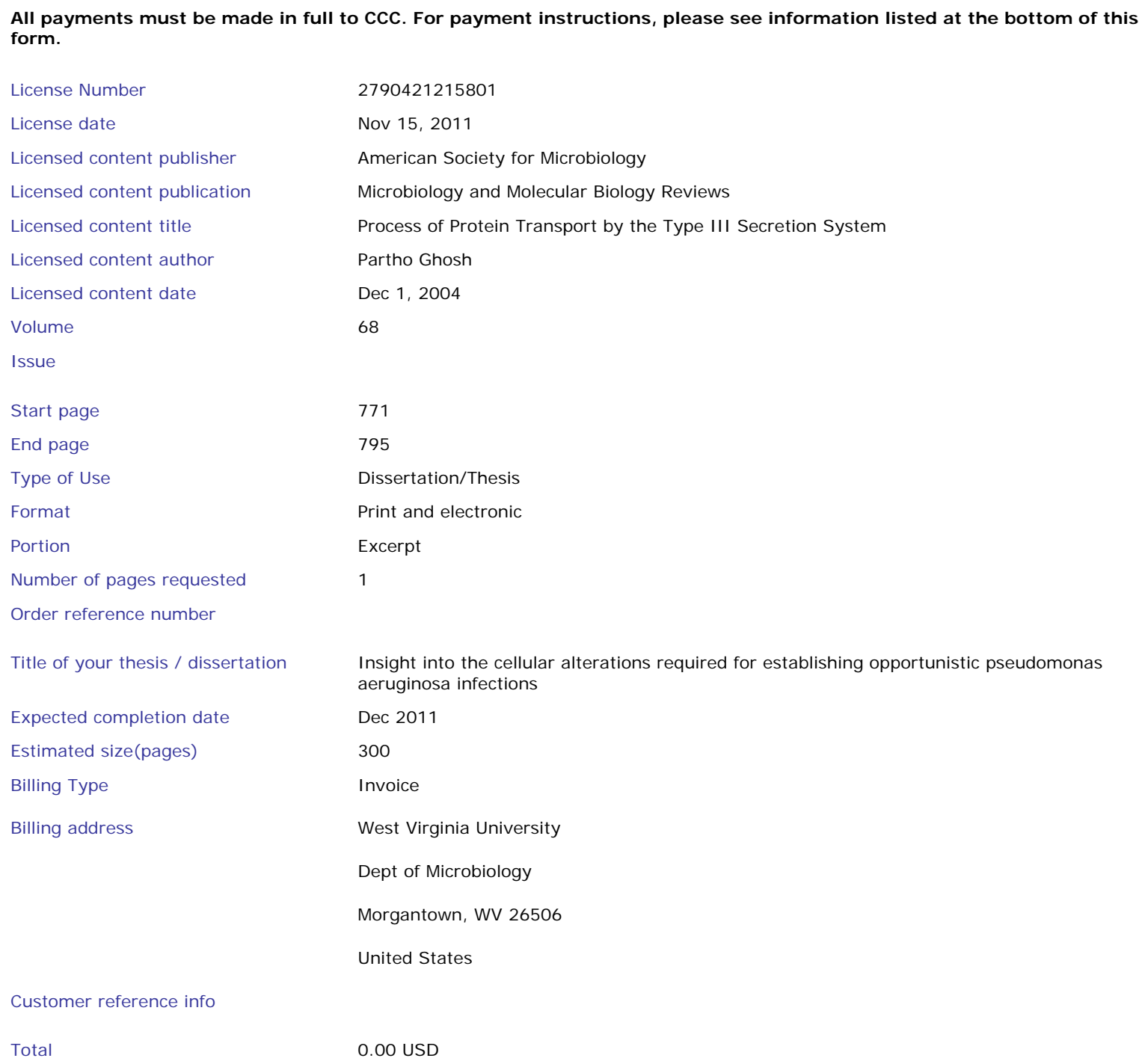

License Number

License date

Licensed content publisher

Licensed content publication

Licensed content title

Licensed content author

Licensed content date

Volume

Issue

Start page

End page

Type of Use

Format

Portion

Number of pages requested

Order reference number

Title of your thesis / dissertation

Expected completion date

Estimated size(pages)

Billing Type

Billing address

Customer reference info

Total

2790421215801

Nov 15, 2011

American Society for Microbiology

Microbiology and Molecular Biology Reviews

Process of Protein Transport by the Type III Secretion System

Partho Ghosh

Dec 1, 2004

68

771

795

Dissertation/Thesis

Print and electronic

Excerpt

1

Insight into the cellular alterations required for establishing opportunistic pseudomonas aeruginosa infections

Dec 2011

300

Invoice

West Virginia University

Dept of Microbiology

Morgantown, WV 26506

United States

0.00 USD 


\section{Figure 4:}

ELSEVI ER LI CENSE

TERMS AND CONDITIONS

Nov 15, 2011

This is a License Agreement between Dacie R Bridge ("You") and Elsevier ("Elsevier") provided by Copyright Clearance Center ("CCC"). The license consists of your order details, the terms and conditions provided by Elsevier, and the payment terms and conditions.

All payments must be made in full to CCC. For payment instructions, please see information listed at the bottom of this form.

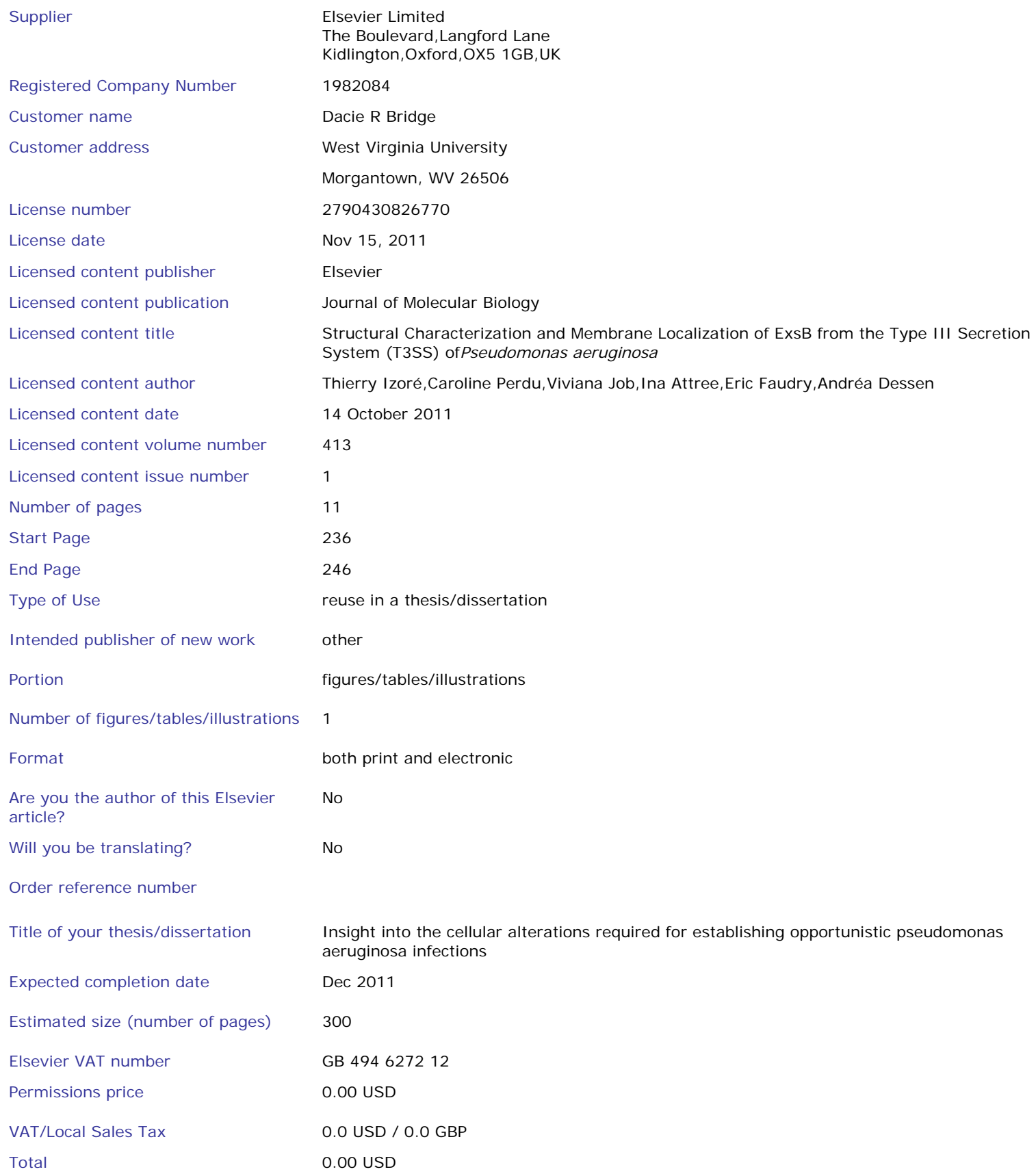


Figure 6:

JOHN WI LEY AND SONS LI CENSE

TERMS AND CONDITIONS

Nov 15, 2011

This is a License Agreement between Dacie R Bridge ("You") and John Wiley and Sons ("John Wiley and Sons") provided by Copyright Clearance Center ("CCC"). The license consists of your order details, the terms and conditions provided by John Wiley and Sons, and the payment terms and conditions.

\begin{tabular}{|c|c|}
\hline License Number & 2790431140754 \\
\hline License date & Nov 15, 2011 \\
\hline Licensed content publisher & John Wiley and Sons \\
\hline Licensed content publication & Respirology \\
\hline Licensed content title & Pseudomonas aeruginosa: Host defence in lung diseases \\
\hline Licensed content author & Bryan J. WILLIAMS, J oanne DEHNBOSTEL,Timothy S. BLACKWELL \\
\hline Licensed content date & Oct 1,2010 \\
\hline Start page & 1037 \\
\hline End page & 1056 \\
\hline Type of use & Dissertation/Thesis \\
\hline Requestor type & University/Academic \\
\hline Format & Print and electronic \\
\hline Portion & Figure/table \\
\hline Number of figures/tables & 1 \\
\hline \multicolumn{2}{|l|}{ Number of extracts } \\
\hline Original Wiley figure/table number(s) & Figure 1 \\
\hline Will you be translating? & No \\
\hline \multicolumn{2}{|l|}{ Order reference number } \\
\hline Total & 0.00 USD \\
\hline
\end{tabular}

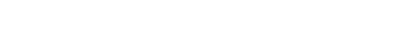

Hagen
DN: $c n=J$ ohn H. Hagen, o=West

DN: $\mathrm{cn}=$ John H. Hagen, $\mathrm{o}=W$
Virginia University Libraries, 